\title{
Natural Gas \\ Imports and Exports \\ Third Quarter Report 2000
}

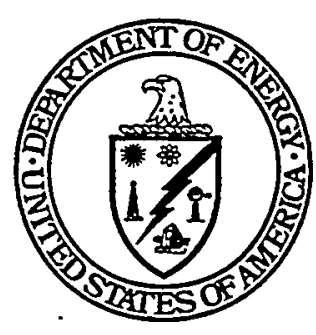

Prepared by:

U.S. Department of Energy

Office of Fossil Energy

Office of Natural Gas and Petroleum

RECEIVED

JAN 122001

OSTI

Import and Export Activities

Washington, DC 20585

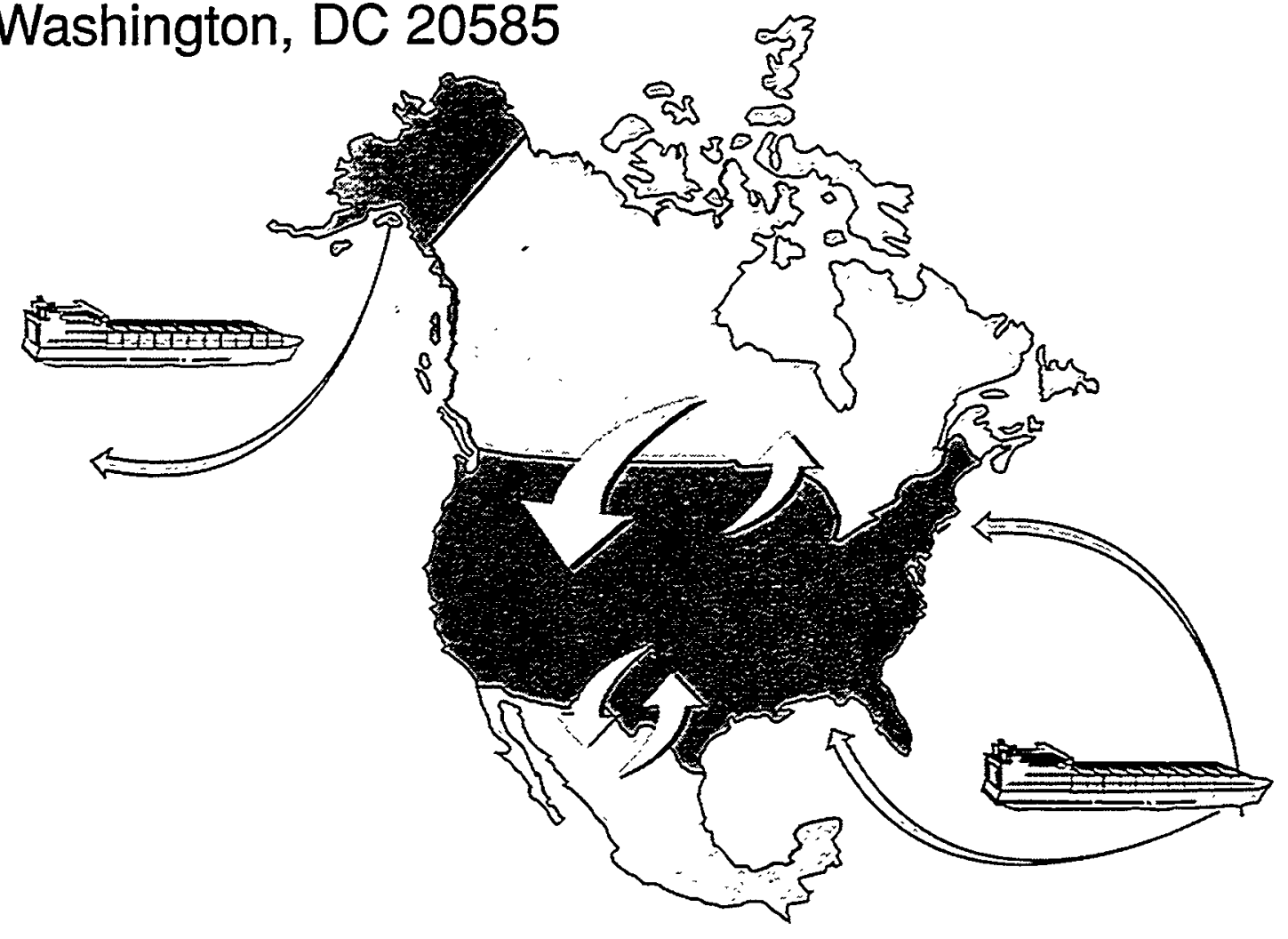

(2) Printed with soy ink on recycled paper 


\section{DISCLAIMER}

This report was prepared as an account of work sponsored by an agency of the United States Government. Neither the United States Government nor any agency thereof, nor any of their employees, make any warranty, express or implied, or assumes any legal liability or responsibility for the accuracy, completeness, or usefulness of any information, apparatus, product, or process disclosed, or represents that its use would not infringe privately owned rights. Reference herein to any specific commercial product, process, or service by trade name, trademark, manufacturer, or otherwise does not necessarily constitute or imply its endorsement, recommendation, or favoring by the United States Government or any agency thereof. The views and opinions of authors expressed herein do not necessarily state or reflect those of the United States Government or any agency thereof. 


\section{DISCLAIMER}

Portions of this document may be illegible in electronic image products. Images are produced from the best available original document. 
The Office of Natural Gas \& Petroleum Import \& Export Activities prepares quarterly reports showing natural gas import and export activity. Companies are required, as a condition of their authorizations, to file quarterly reports. This report is for the 3rd quarter of 2000 (July - Sept.).

Attachment A shows the percentage of takes to maximum firm contract levels and the weighted average per unit price for each of the long-term importers during the 5 most recent reporting quarters.

Attachment $B$ shows volumes and prices of gas purchased by long-term importers \& exporters during the past 12 mos. Attachment $\mathrm{C}$ shows volume and price data for gas imported on a short-term or spot market basis.

Attachment $D$ shows the gas exported on a short-term or spot market basis to Canada and Mexico.

Third Quarter Highlights: As shown below, there were notable changes in activity compared to 3rd quarter 1999.

\begin{tabular}{|lrl|}
\hline Canadian Imports & $871.6 \mathrm{Bcf}$ & up 5\% \\
LNG Imports & $73.6 \mathrm{Bcf}$ & up 55\% \\
Mexican Imports & $0.2 \mathrm{Bcf}$ & down $98 \%$ \\
Total Imports & $945.4 \mathrm{Bcf}$ & up $6 \%$ \\
Mexican Exports & $31.7 \mathrm{Bcf}$ & up $95 \%$ \\
Canadian Exports & $12.6 \mathrm{Bcf}$ & up $77 \%$ \\
Japanese Exports & $16.8 \mathrm{Bcf}$ & same \\
Total Exports & $61.1 \mathrm{Bcf}$ & up 52\% \\
\hline
\end{tabular}

See details below for more information.

$\begin{array}{lll}\text { Puerto Rico Imports } \quad 6.6 \mathrm{Bcf} & \text { New Project }\end{array}$

Puerto Rico Imports: This calendar quarter marks the first time LNG imports into Puerto Rico have been reported. These imports are shown in the Table above, the quarterly summary page showing imports by type of DOE imports authorization (on next page), and in Attachment B-1 (p. 22). However, the imports are not reflected in any of the summary totals found on this page, as the summaries are only for trade pertaining to continental U.S.

There were delays in the commercial start-up of the Alliance Pipeline but it became operational on December 1,2000 . Alliance imported $4.62 \mathrm{Bcf}$ in the third quarter for testing purposes.

EcoElectrica, L.P. imported LNG from Trinidad \& Tobago into Puerto Rico for the first time in July. A total of $6.6 \mathrm{Bcf}$ was imported in the 3rd quarter. The LNG is used to fuel a 461-megawatt combined-cycle cogeneration plant on the southern coast of Puerto Rico near the city of Ponce.

During the third quarter, seven importers of $L N G$ (Distrigas Corporation; Duke Energy LNG; CMS Marketing Services \& Trading Co.; Sempra Energy Trading Corporation; Coral Energy Resources, L.P.; Enron International Gas Sales Co.; and Amoco Energy Trading Corporation) brought in 32 cargoes from six countries (Algeria, Australia, Nigeria, Qatar, Trinidad, and Oman), totaling 73.6 Bcf.

Third Quarter Data: Comparing 3rd quarter 2000 to 3rd quarter 1999 data, total imports under long-term contracts declined slightly. Specifically, natural gas imports under all long-term contracts totaled $357.5 \mathrm{Bcf}$, compared to $358.8 \mathrm{Bcf}$ in the third quarter of 1999. Of this total, longterm Canadian imports were down 2 percent (328.6 v. $334.1 \mathrm{Bcf}$ ). The average price of this gas was $\$ 3.83 \mathrm{per}$
MMBtu, which is 68 cents higher than the preceding quarter and $\$ 1.39$ or 57 percent higher than the third quarter of 1999. Long-term LNG imports increased by 28.9 Bcf. Under LNG long-term imports, Distrigas imported 2.4 Bcf from Algeria at an average price of $\$ 3.44$ per MMBtu and 22.1 Bcf from Trinidad at \$2.98 per MMBtu. Duke imported 4.4 Bcf from Algeria at $\$ 3.33$ per MMBtu.

During the third quarter, 85 companies used short-term authorizations to import $588.0 \mathrm{Bcf}$ of gas, which is a 6.5 percent increase over the third quarter of 1999 (552.1 Bcf). Of this total, 543.1 Bcf was imported from Canada at an average price of $\$ 3.88$ per MMBtu, compared to $513.8 \mathrm{Bcf}$ at $\$ 2.30$ in the third quarter of 1999 , and $496.8 \mathrm{Bcf}$ at $\$ 3.25$ in the previous quarter. Imports from Mexico totaled 0.2 Bcf at an average price of $\$ 4.88$, compared to $14.5 \mathrm{Bcf}$ at $\$ 2.44$ in the third quarter of 1999 and $0.8 \mathrm{Bcf}$ at $\$ 2.97$ last quarter. Short-term LNG imports totaled $44.7 \mathrm{Bcf}$ for the quarter, compared to 26.2 Bcf last quarter. Under shortterm LNG import contracts, Duke imported 0.7 Bcf from Algeria at an average price of $\$ 3.33$ per MMBtu; Sempra imported 3.7 Bcf from Trinidad at \$3.39 per MMBtu; Amoco imported 1.8 Bcf from Trinidad at $\$ 3.80$ per MMBtu; and Enron imported 2.7 Bcf from Oman at \$3.58 per MMBtu. CMS imported 3.6 Bcf from Australia at $\$ 2.79$ per MMBtu; 19.6 Bcf from Qatar at \$3.26 per MMBtu; and 2.5 Bcf from Nigeria at $\$ 4.37$ per MMBtu. Coral imported $4.9 \mathrm{Bcf}$ from Oman at \$2.44 per MMBtu and 5.2 Bcf from Nigeria at $\$ 3.18$ per MMBtu.

Approximately 30 percent of the short-term Canadian imports occurred at Eastport, ID at an average price of \$3.74 per MMBtu; 24 percent at Port of Morgan, MT at $\$ 3.76 ; 11$ percent at Sumas, WA at $\$ 3.47 ; 11$ percent at Noyes, MN at $\$ 4.04 ; 10$ percent at Niagara Falls, NY at $\$ 4.32$; 6 percent at Calais, ME at $\$ 4.31 ; 4$ percent at Waddington, NY at \$4.35; and 4 percent at other entry points at $\$ 4.09$.

In addition, 21 short-term export authorizations were used, exporting a total of $44.3 \mathrm{Bcf}$ of gas. Eight companies exported 12.6 Bcf to Canada, at an average.price of $\$ 4.29$ per MMBtu. Thirteen companies exported $31.7 \mathrm{Bcf}$ to Mexico at $\$ 4.57$ per MMBtu. Finally, 16.8 Bcf of LNG was exported to Japan at $\$ 4.28$ per MMBtu (delivered).

Year to Date Data: Comparing the first 9 months of 2000 with the first 9 months of 1999, total gas imports increased 4 percent $(2,755.9$ v. 2,652.3 Bcfi) and total gas exports increased 44 percent ( 174.9 v. $121.7 \mathrm{Bct})$. Canadian imports increased by 97 Bcf or nearly 4 percent $(2,589.5 \mathrm{v}$. 2,490.8 Bcf); Mexican imports decreased 88 percent (5.0 v. $40.6 \mathrm{Bcf}$ ); and LNG imports increased 33 percent (161.4 v. 120.9 Bcf). Exports to Canada increased 97 percent (48.9 v. $24.8 \mathrm{Bcf}$ ) and exports to Mexico increased 64 percent (79.2 v. 48.2 Bcf). LNG exports to Japan decreased 4 percent ( 46.8 v. $48.7 \mathrm{Bcf}$ ).

This quarter's focus report is "Recent Developments and Trends in Cross-Border Gas Trade." The quarterly report and future revisions to the report will reside on our Electronic Bulletin Board at (202) 586-7853 and on the Fossil Energy Web Site at http://www.fe.doe.gov (click on Regulatory - Natural Gas). Any questions or comments about this report should be directed to Yvonne Caudillo at (202) $586-4.587$ or by E-mail at yvonne.caudillo@hq.doe.gov. 
Eastport, Idaho

$47,568.00$

Everett, Massachusetts

$24,494.96$

3.30

Grand Island, New York

$3,238.89$

$3.03 *$

Highgate Springs, Vermont

$1,055.36$

4.06

International Falls, Minnesota

Lake Charles, Louisiana

Massena, New York

Niagara Falls, New York

$42,449.06$

4.63

Noyes, Minnesota

$57,910.16$

3.94

Pittsburg, New Hampshire

$3,478: 68$

$6,628.59$

Ponce, Puerto Rico

$77,924.07$

126.90

Portal, North Dakota

St. Clair, Michigan

$1,863.64$

$22,084.51$

$60,969.29$

3.69

4.40

$3.14 *$

3.80

1.94

3.84

3.33

Waddington, New York

24.95

4.41

Warroad, Minnesota

Babb, Montana

Calais, Maine

Champlain, New York

Eastport, Idaho

Grand Island, New York

Havre, Montana

Hidalgo, Texas

Highgate Springs, Vermont

Lake Charles, Louisiana

Massena, New York

Niagara Falls, New York

Noyes, Minnesota

Pittsburg, New Hampshire

Port of Del Bonita, Montana

Port of Morgan, Montana

Sherwood, North Dakota

St. Clair, Michigan

Sumas, Washington

Waddington, New York

Warroad, Minnesota

Whitlash, Montana

\section{SHORT-TERM AUTHORIZATIONS}

$\begin{array}{rl}54.64 & 3.49 \\ 35,079.64 & 4.31 \\ 0.58 & 4.55 \\ 160,915.26 & 3.74 \\ 7,043.50 & 4.51 \\ 997.21 & 3.34 \\ 245.89 & 4.88 \\ 408.95 & 4.69 \\ 44,651.91 & 3.20^{* *} \\ 746.12 & 4.45 \\ 55,740.63 & 4.32 \\ 59,119.32 & 4.04 \\ 2,608.11 & 3.40 \\ 22.32 & 4.78 \\ 127,893.97 & 3.76 \\ 4,620.20 & 4.39 \\ 1,485.43 & 4.49 \\ 60,337.82 & 3.47 \\ 21,169.33 & 4.35 \\ 1,721.78 & 3.67 \\ 3,101.51 & 3.46\end{array}$

$587,964.11$

* Average landed price

** Average tailgate price

Note: Import figures in this table may vary slightly from sum of components in report due to independent rounding in calculations. 


\section{QUARTERLY FOCUS:}

\section{RECENT DEVELOPMENTS AND TRENDS IN CROSS-BORDER GAS TRADE}

\section{INTRODUCTION}

This Focus Feature provides a review of some of the more notable developments and trends in cross-border natural gas trade during the first nine months of 2000. It specifically looks at the major pipeline infrastructure improvements which have become operational during this period and compares the volume and price data for this period with the same time period in 1999. This Focus Feature is comprised of three sections. The First Section examines natural gas import and export trade with Mexico and any new pipeline construction developments which would facilitate increased trade between Mexico and the United States. The Second Section looks at imports of liquefied natural gas (LNG) for the first nine months of trade and compares the figures with the same period in 1999. The Third Section examines natural gas import and export activity between Canada and the United States for the first nine months of 2000 and looks at two pipeline projects which markedly increase the ability of Canada to supply additional volumes of natural gas to the growing U.S. market.

\section{U.S.-Mexico Natural Gas Trade}

Improved Pipeline Infrastructure at the International Border

Over the past few years, natural gas use in Mexico has become an increasingly important energy source. Most gas demand forecasts for Mexico project that the annual growth rates will be between 8 and 10 percent during the foreseeable future. In order to supply this anticipated growth in demand, there have been substantial investments made in the natural gas pipeline infrastructure. During 2000, there has been continued expansion and improvements of the natural gas pipeline infrastructure along the United States and Mexico border. Figure 1 on the next page shows the general location of the ten existing natural gas pipeline interconnects between the United States and Mexico. Figure 1 also includes a table indicating the locations of the pipelines on the international border, their estimated design capacities, and their estimated average daily throughputs from 1992 through the first nine months of 2000 . With the construction and operation of two new pipelines, aggregate natural gas pipeline capacity at the international border grew by 600 million cubic feet per day (MMcf/d) during the year, increasing from 1370 to $1970 \mathrm{MMcf} / \mathrm{d}$; this represents an increase in total pipeline capacity of almost 44 percent. The two completed pipeline projects, shown in Figure $\mathbf{I}$ as $\mathrm{A}$ and $\mathrm{J}$, are discussed below.

Rosarito Pipeline Project: Sempra Energy International (Sempra), and its utility affiliate San Diego Gas and Electric, constructed a new international gas pipeline facility at Otay Mesa, San Diego County, California for the export of natural gas to Mexico. The portion of the facility located on the U.S. side of the border consists of a meter station and 400 feet of 30 -inch pipeline leading from the meter station to the international border. At the international border, Sempra constructed a 23-mile pipeline to the Presidente Juarez power plant in Rosarito, Baja California, south of Tijuana.

Natural gas began flowing on this pipeline in April 2000. The chart below shows the gradual growth in the utilization of this pipeline since its initial start-up.

Average Daily Throughput During the

First 6 Months of Operation (MMcf)

April May June $\underline{\text { July }}$ August $\underline{\text { Sept. }}$

\begin{tabular}{llllll}
0.11 & 0.62 & 1.6 & $44.8^{\circ}$ & 69.1 & 62.3 \\
\hline
\end{tabular}




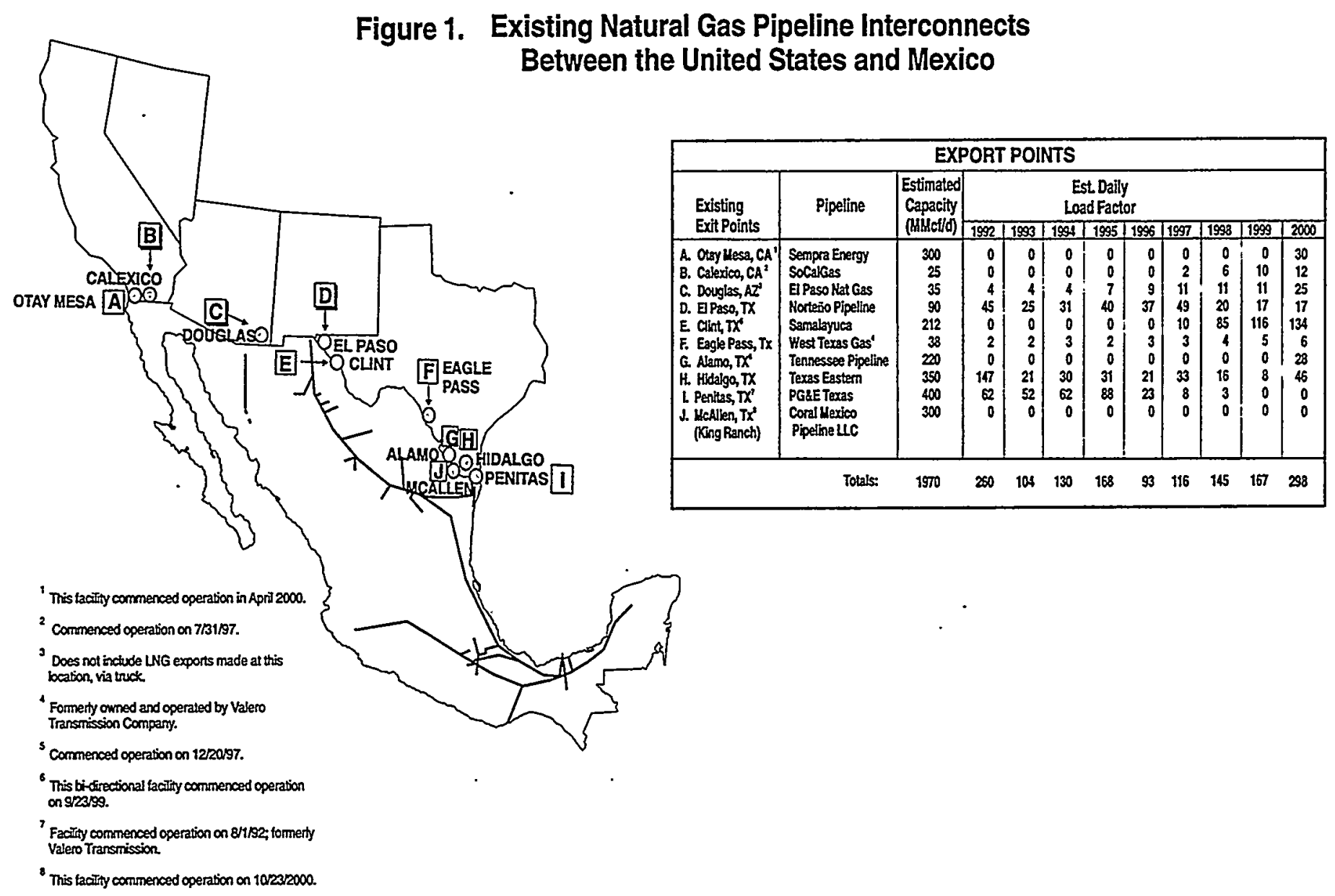

Sources: Data derived from quartety reponts hied with Fossl Energy by natural gas exponers and fings before the FERC.

According to Sempra, natural gas deliveries through this pipeline will allow the power plant to switch from oil to natural gas use and expand its generating capacity to meet the rapidly growing energy demand in Baja California from the manufacturing and residential sectors. Sempra has a 10-year gas supply contract with the Mexican Federal Electric Commission (CFE) to deliver up to $300 \mathrm{MMcf} / \mathrm{d}$ of natural gas to its Rosarito power plant. In addition to serving CFE's power plant, this new pipeline will make natural gas available for the first time to businesses and residents in nearby cities, as additional distribution systems are being planned to serve Tijuana, Tecate, and Ensenada.

Coral Mexico Pipeline: Coral Mexico Pipeline, LLC (Coral), a subsidiary of Tejas Energy LLC, built a 24-inch, 104-mile, $300 \mathrm{MMcf} / \mathrm{d}$ capacity bi-directional pipeline which will connect its system in Texas with the Pemex system in Mexico. This pipeline extends from Coral's pipeline system on the King Ranch in south Texas to the Mexican border near McAllen, Texas. This new pipeline also includes 1.5 miles of new pipeline in Mexico which interconnects with the Pemex system at Arguielles, in the Mexican State of Tamualipas. Although Coral built the entire pipeline, Pemex owns and operates the portion in Mexico.

Coral began flowing natural gas to Mexico through this new pipeline on October 23, 2000. This $\$ 50$ million pipeline, which features a bidirectional design, enables the flow of gas in either direction across the border. This pipeline, 
along with others on the U.S.-Mexico border with bi-directional capabilities, i.e., Tennessee, Texas Eastern, has the flexibility to adapt to changing market conditions in both countries.

The outlook for further natural gas pipeline expansions along the U.S.- Mexico border in 2001 indicates that there will be limited construction, but the trend toward closer system integration between the two countries will continue. Discussed below are two projects which are expected to be completed during the upcoming year.

El Paso's Wilcox Lateral Project: On February 9, 2000, the Federal Energy Regulatory Commission (FERC) issued a Presidential Permit and Certificate of Public Convenience and Necessity authorizing El Paso Natural Gas Company (EPNG) to construct and operate the proposed Willcox Lateral facilities in Cochise County, Arizona (CP99-322,323). The EPNG Willcox Lateral consists of 56 miles of a 20 -inch pipeline constructed downstream from its California mainline where it will separate into two 16-inch branch lines that will end about 15 miles apart at the U.S./Mexican border. This project will add an additional $130 \mathrm{MMcf} / \mathrm{d}$ of pipeline capacity at the international border. EPNG is planning to construct the western branch during the next few months in order for it to be operational by April 2001. This new pipeline, which will be constructed near Naco, Arizona, will be used to supply natural gas to two electric power plants located at Hermosillo, Mexico - - an existing 250-MW peaking unit owned and operated by the Comision Federal de Electricidad (CFE) and another 225-MW combined-cycle power plant that is currently being built by a subsidiary of Spain's Union Fenosa Group. El Paso Merchant Energy Co. has a 25-year contract with Union Fenosa to supply it with up to 43 MMcf/day, beginning in April 2001. In order to move firm supplies to Hermosillo, EPNG must build the lateral and a compressor station on the Pemex system. The eastern branch of the Wilcox project likely will not be completed until 2003 when it will be needed to supply a yet-to-be built 250-MW power plant located near Agua Prieta. This power plant also will be constructed and operated by Union Fenosa.
Capacity Expansion on Samalayuca Pipeline: EPNG is planning to add additional compression on the Mexican side of the border where the Samalyuca Pipeline interconnects with the pipeline system owned and operated by Pemex (See Fig. 1). Initially, the added compression will add about $60 \mathrm{MMcf} / \mathrm{d}$ of pipeline capacity to the already existing $212 \mathrm{MMcf} / \mathrm{d}$ capacity at the international border near Clint, Texas. The new capacity is needed to serve a new 250-MW power plant currently being build by CFE at Chihuahua. Approximately $40 \mathrm{MMcf} / \mathrm{d}$ of natural gas will be needed to supply this gas-fired power plant by April 2001. According to EPNG, additional compression and pipeline capacity will needed in the future when a couple of other planned power plants are constructed in the area during the next few years.

\section{U.S.-Mexico Natural Gas Trade During the First Nine Months of 2000}

As mentioned above, natural gas demand in Mexico continues to show robust growth, paced largely by the increased use of natural gas to fuel electric power generation. As noted above in the section describing new and planned trans-border pipelines, virtually all of the construction projects were designed primarily to supply natural gas to fuel the power sector, either for newly constructed gas-fired power plants, or existing ones which have been converted from oil to gas use. The two Tables shown below compare the first nine months of gas trade between the U.S. and Mexico in 2000 with what was experienced during the same time period in 1999. As shown in Table 1, natural gas imports from Mexico during the first 9 months of 2000 dropped 88 percent compared with the import level experienced during first 9 months of 1999: $5.0 \mathrm{v} .40 .6 \mathrm{Bcf}$. The year-to-year drop in natural gas imports is undoubtedly attributable to the rapid rise in domestic demand and a relatively unchanged gas production rate. For the first 9 months of 2000, the weighted average price at the international border for gas imports was $\$ 2.58$ per MMBtu; compared with $\$ 2.09$ per MMBtu during the first 9 months of 1999. This represents a 23 percent year-to-year increase. 
North American Gas Trade * North American Gas Trade * North American Gas Trade * North American Gas Trade * North American Gas Trade

Natural Gas Imports from Mexico: First 9 Months (1999 \& 2000)

Table 1

\begin{tabular}{|c|c|c|c|c|c|c|c|c|c|c|}
\hline \multicolumn{11}{|c|}{ Volume: Billion Cubic Feet } \\
\hline & Jan. & Feb. & March & April & May & June & July & Aug. & Sept. & Total \\
\hline 1999 & 4.9 & 4.4 & 0.8 & 4.2 & 6.8 & 5.0 & 3.9 & 6.0 & 4.6 & 40.6 \\
\hline 2000 & 2.9 & 0.7 & 0.3 & 0.8 & 0.0 & 0.0 & 0.03 & 0.01 & 0.2 & 5.0 \\
\hline \multicolumn{11}{|c|}{ Price: $\$ / M M B t u$} \\
\hline & Jan. & Feb. & March & April & May & June & July & Aug. & Sept. & Wt. Avg Price \\
\hline 1999 & 1.74 & 1.69 & 1.59 & 2.01 & 1.94 & 2.11 & 2.21 & 2.61 & 2.39 & $\$ 2.09$ \\
\hline 2000 & 2.30 & 2.50 & 2.60 & 2.97 & 0.00 & 0.00 & 4.01 & 4.64 & 5.00 & $\$ 2.58$ \\
\hline
\end{tabular}

Table 2 compares the monthly volume and price data for natural gas exports to Mexico during the first 9 months of 2000 and 1999. As shown, 2000 natural gas exports increased by $31^{\prime} \mathrm{Bcf}, \cdots$ or 64 percent over the 1999 level. The weighted average international border price for gas sales to Mexico during the first 9 months of 2000 was $\$ 3.69$ per MMBtu, or a $\$ 1.50$ per unit increase over last year's average price of $\$ 2.19$ per MMBtu during the same time period. This represents an increase of over 68 percent. Consistent with the recent price volatility experienced throughout the North American gas market this year, the price of gas exports jumped 110 percent between January and September of this year (\$2.41 v. \$5.07 per MMBtu).

Comparing the first 9 months of 2000 with the same time period last year, the $35.6 \mathrm{Bcf}$ decline in natural gas imports from Mexico, coupled with a $31 \mathrm{Bcf}$ increase in gas exports, has resulted in a $66.6 \mathrm{Bcf}$ swing in our gas trade with Mexico so far this year.

Natural Gas Exports to Mexico: First 9 Months (1999 \& 2000)

Table 2

\begin{tabular}{|c|c|c|c|c|c|c|c|c|c|c|}
\hline \multicolumn{11}{|c|}{ Volume: Billion Cubic Feet } \\
\hline & Jan. & Feb. & March & April & May & June & July & Aug. & Sept. & Total \\
\hline 1999 & 4.6 & 4.8 & 6.0 & 5.1 & 6.1 & 5.3 & 5.6 & 5.4 & 5.3 & 48.2 \\
\hline 2000 & 6.0 & 6.4 & . 7.7 & 8.3 & 10.4 & 8.7 & 10.1 & 11.3 & 10.3 & 79.2 \\
\hline \multicolumn{11}{|c|}{ Price: $\$ /$ MMBtu } \\
\hline & Jan. & Feb. & March & April & May & June & July & Aug. & Sept. & Wt. Avg Price \\
\hline 1999 & 1.84 & 1.76 & $1.64 \cdot \cdot$ & 1.89 & $2: 29$ & 2.31 & 2.33 & 2.72 & 2.91 & $\$ 2.19$ \\
\hline 2000 & 2.41 & 2.63 & 2.72 & 2.95 & 3.24 & 4.30 & 4.52 & 4.17 & 5.07 & $\$ 3.69$ \\
\hline
\end{tabular}


North American Gas Trade * North American Gas Trade * North American Gas Trade * North American Gas Trade * North American Gas Trade

\section{LNG Import Trade}

Start-Up of New Import Project in Puerto Rico: EcoElectrica, L.P.

On July 10, 2000, Puerto Rico received its first shipment of LNG to fuel a new 461-MW, gasfired cogeneration plant built by EcoElectrica, L.P. The new EcoElectrica facility is located on the southern coast in Guayanilla Bay near the city of Ponce and is owned by affiliates of Enron Corporation and Edison International. The $\$ 670$ million LNG Project consists of a marine unloading terminal, two LNG storage tanks (million bbl. capacity each), a vaporization system, and a natural gas accumulator pipeline. In addition, the power plant facility consists of two gas combustion turbines and a steam turbine, as well as its own water desalination plant to produce potable water for use in the power plant and to supplement the public water supplies in the area. The electricity generated by EcoElectrica's cogeneration facility is sold under a, 22-year sales agreement to the Puerto Rico Electric Power Authority (PREPA), the government-owned public utility that supplies almost all of the electric power consumed on the island. This project is a part of PREPA's strategy to diversify its power generation sources because of the island's historical high reliance on oil-burning generation plants. During its first 65 days of operation (July 10 - September 13), EcoElectrica purchased 3 cargoes of LNG from Cabot LNG Trading Limited, totaling $6.6 \mathrm{Bcf}$ : All of the supplies came from Trinidad \& Tobago under a long-term purchase contract (see Table 3 on next page).

In another effort to diversify its generation sources, PREPA has signed a 25-year purchase agreement to purchase electricity from a new coalfired cogeneration facility being built by AES Corporation (AES). AES began construction of a 454-MW plant on the south coast in November 1999 and expects that the facility will be operational by mid-2002. Like the EcoElectrica Project, this facility will supply about 15 percent of the island's current electricity demand.
Cabot Corporation Sells LNG Unit to Tractebel, Inc.

On September 19, 2000, Cabot Corporation announced the completion of its sale of Cabot LNG, LLC to Tractebel, Inc., for $\$ 680$ million. Tractebel is a global energy and services business and is the sole energy marketing arm of Suez Lyonnaise des Eaux. Cabot LNG owns and operates the only active LNG import terminal on the East Coast. The facility, located at Everett, Massachusetts (just north of Boston), has been in operation since 1971. In 1999, Distrigas imported $96.1 \mathrm{Bcf}$ of LNG and supplied approximately 17 percent of the natural gas demand in New England. Distrigas estimates that it will import 99 Bcf of LNG during calendar 2000. With the completion of the Distrigas sale, there has been a transfer in ownership of all four LNG import terminals in the U.S. over the last two years.

\section{LNG Import Trade During the First Nine Months of 2000}

During the first 9 months of the year, eight companies, led by Distrigas Corporation, CMS Marketing, Services and Trading Co., and Duke Energy LNG Sales, Inc., imported a total of 161.4 Bcf of LNG into the United States. As noted above, EcoElectrica of Puerto Rico (P.R.) started receiving LNG in July from Trinidad \& Tobago at its new LNG receiving terminal on its south coast near the city of Ponce; it has imported $6.6 \mathrm{Bcf}$ since its commercial start-up. LNG imports into the U.S. for the first 9 months of 2000 increased 40.5 Bcf over the same 9-month period in 1999 $(161.4$ v.120.9 Bcf), or a rise in import activity of 33 percent. Table 3 below shows the LNG imports into the United States and Puerto Rico by country of origin and receiving terminal. Table 3 also shows four recent trends in LNG import trade: (1) the number of countries supplying LNG to the U.S. has grown dramatically in the past 5 years -- in 1995, Algeria was the sole supplier; today, LNG imports come from 7 different countries; (2) import volumes continue to climb, growing from an annual figure of $18 \mathrm{Bcf}$ in 1995 to topping $200 \mathrm{Bcf}$ this year for the $1^{\text {st }}$ time since 
1979; (3) the rapid expansion of a LNG spot market, with almost $50 \%$ of the import volumes for 2000 being made under spot market sales arrangements; and (4) the increased use of the Lake Charles terminal - - a vibrant spot market likely will make this busiest import facility during the year.
As shown in Table 3, Trinidad \& Tobago has replaced Algeria as the largest supplier of LNG during the first 9-months of the year. In addition, Qatar, solely on the basis of spot market sales, became the $2^{\text {nd }}$ largest foreign supplier of LNG during this time period.

\section{LNG IMPORTS INTO THE UNITED STATES AND THE COMMONWEALTH OF PUERTO RICO BY COUNTRY OF ORIGIN AND RECEIVING TERMINAL (FIRST 9 MONTHS OF 2000)}

Table 3

\begin{tabular}{|c|c|c|c|c|c|c|}
\hline $\begin{array}{c}\text { Country of } \\
\text { Origin }\end{array}$ & $\begin{array}{c}\text { Number of } \\
\text { Cargoes }\end{array}$ & $\begin{array}{c}\text { Everett } \\
\text { MA }\end{array}$ & $\begin{array}{c}\text { Lake Chas. } \\
\text { LA }\end{array}$ & $\begin{array}{l}\text { Ponce } \\
\text { P.R. }\end{array}$ & $\begin{array}{l}\text { U.S. } \\
\text { Total }\end{array}$ & $\begin{array}{c}\text { U.S. \& } \\
\text { P.R. Total }\end{array}$ \\
\hline \multicolumn{7}{|c|}{ Volumes in Bcf } \\
\hline Algeria & $12(0)$ & 11.3 & 17.7 & & 29.0 & 29.0 \\
\hline Australia & $3(3)$ & & 5.8 & & 5.8 & 5.8 \\
\hline Nigeria & $4(4)$ & & 10.2 & & 10.2 & 10.2 \\
\hline Oman & $3(3)$ & & 7.7 & & 7.7 & 7.7 \\
\hline Qatar & $13(13)$ & & 31.7 & & 31.7 & 31.7 \\
\hline $\begin{array}{l}\text { Trinidad \& } \\
\text { Tobago }\end{array}$ & $38(11)$ & 60.1 & 14.2 & 6.6 & 74.3 & 80.9 \\
\hline UAE & $1(1)$ & & 2.7 & & 2.7 & 2.7 \\
\hline TOTAL & $74(36)$ & 71.4 & 90.0 & 6.6 & 161.4 & 168.0 \\
\hline
\end{tabular}

Note: Parentheses indicate spot market cargoes. 
Figure 2 illustrates the growth of LNG imports over the past 21 months (Jan. 1999 - Sept. 2000) by providing the average daily LNG imports coming into the United States by month and by receiving terminal. As noted earlier, LNG imports during the first 9 months of 2000 increased by $40.5 \mathrm{Bcf}$ over the same time period last year. LNG imports by Distrigas into its Everett, Massachusetts, facility this year are virtually the same as last year; as a consequence, the entire growth experienced this year is the result of increased volumes coming into CMS Energy's Lake Charles LNG facility - - all short-term, spot market transactions. For the $3^{\text {rd }}$ calendar quarter of 2000 (July-Sept.), LNG imports averaged 800 MMcf/day -- $534 \mathrm{MMcf} /$ day coming into Lake Charles terminal and $266 \mathrm{MMcf} /$ day coming into Everett terminal.

Figure 2

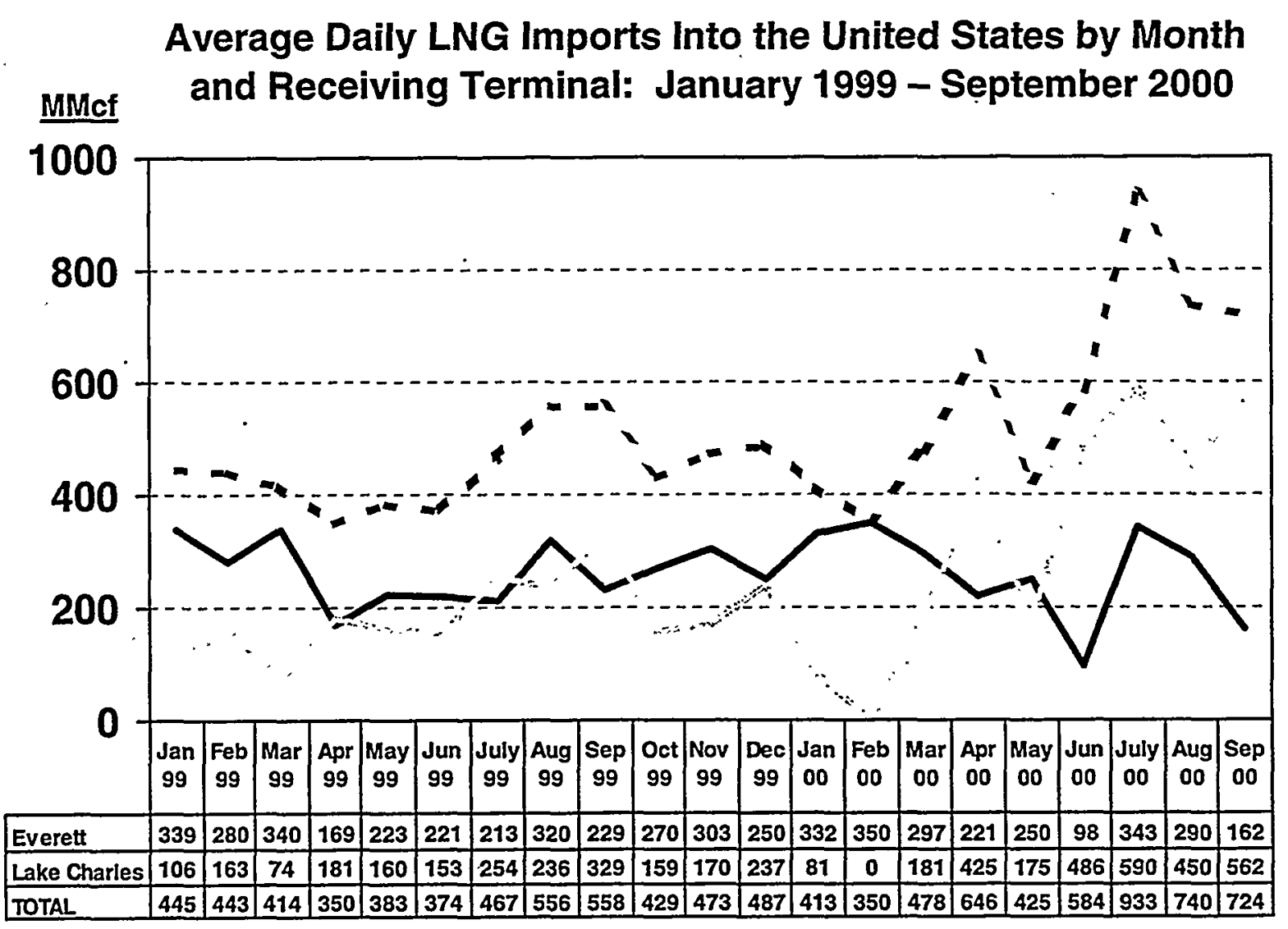


North American Gas Trade * North American Gas Trade * North American Gas Trade * North American Gas Trade** North American Gas Trade

\section{U.S.-Canada Natural Gas Trade}

\section{Start-up of Two New Pipelines to Ship} Canadian Gas Imports into the United States

Maritimes \& Northeast Pipeline (Maritimes): U.S./Canadian cross-border pipeline capacity continues to grow in 2000. The first of two gas pipelines that came on-line this year was the Maritimes, which commenced operation in January. This pipeline transports natural gas from the Sable Island Offshore Energy Project, a new natural gas basin offshore Nova Scotia, to markets in the Atlantic Provinces and New England. The entire Maritimes pipeline system, which extends from a gas plant at Goldboro, Nova Scotia, to Wells, Maine, is about 800 miles long. From the international border near Calais,Maine, Maritimes travels to Westbrook,Maine (near Portland), where it interconnects with pipeline facilities of the Granite State Gas Transmission System and the pipeline jointly owned by Maritimes and the Portland Natural Gas Transportation System (PNGTS). Maritimes currently has an international border pipeline capacity of about $440 \mathrm{MMcf} /$ day, but could be expanded rather easily with the installation of additional compression. The chart below shows the growth in the utilization of this new pipeline facility since its initial start-up at the beginning of the year. During the last two months shown in the chart, the load factor for the pipeline reached over 94 percent. All of the gas flowing into the United States on this pipeline was consumed in the New England area, including many areas in Maine that have never before been served by natural gas.

\begin{tabular}{|c|c|c|c|c|c|c|c|c|}
\hline \multicolumn{1}{|c|}{$\begin{array}{c}\text { Average Daily Throughput During } \\
\text { for Maritimes \& } 9 \text { Months of } 2000 \\
\text { for Noast Pipeline (MMcf) }\end{array}$} \\
\hline Jan. & Feb. & March & April & May & June & July & August & Sept. \\
\hline 32 & 134 & 259 & 323 & 322 & 358 & 387 & 427 & 416 \\
\hline
\end{tabular}

On October 10, 2000, Maritimes filed an. application (CP01-4-00) with the Federal Energy Regulatory Commission (FERC) requesting approval to expand its pipeline system by building and operating two new pipelines in eastern Massachusetts - - Maritimes III and Algonquin's HubLine. Maritimes' proposed system expansion is designed to link its facilities with those of Algonquin and thereby enabling it to transport up to $360 \mathrm{MMcf} /$ day of gas to electric generating facilities, industrial users and local distribution companies located in the eastern part of Massachusetts. Maritimes is hoping to have this proposed expansion operational by November 2002.

Alliance Pipeline: The second cross-border pipeline facility to become operational in 2000 is the Alliance Pipeline (Alliance), which had a commercial start-up date of December 1 . Alliance, a $\$ 4.7$ billion pipeline originating near Fort St. John, British Columbia, extends about 1900 miles to a terminus near Chicago, Illinois. The system extends across the main gas producing regions of Alberta, through Saskatchewan, to a point on the international border near Sherwood, North Dakota. From Sherwood, the pipeline travels across parts of Minnesota, Iowa and northern Illinois. Alliance currently has an international border capacity of 1,325 MMcf/day, but could be easily expanded to $2,000 \mathrm{MMcf} /$ day with installation of additional compression. Alliance, originally scheduled to begin commercial operation on October 1, encountered problems with construction debris in its system while undergoing testing of the facilities. However, the problems seemingly have been resolved, as there are reports that Alliance is 
North American Gas Trade * North American Gas Trade * North American Gas Trade * North American Gas Trade * North American Gas Trade

running at near design capacity in December. The chart below shows the average daily throughput on the Alliance during the first 5 months of testing the system.

\begin{tabular}{|ccccc|}
\hline \multicolumn{5}{|c|}{ Average Daily Throughput During the } \\
5-Months of Testing Alliance Pipeline (MMcf) \\
June & $\underline{\text { July }}$ & $\underline{\text { August }}$ & $\underline{\text { Sept. }}$ & $\underline{\text { Oct. }}$ \\
4.4 & 0 & 25.8 & 127.3 & 369.3 \\
\hline
\end{tabular}

\section{Canadian Gas Import/Export Trade During the First 9 Months of 2000}

Comparing the first nine months of 2000 with the same period in 1999, natural gas imports from Canada have grown by $98.7 \mathrm{Bcf}(2,589.6 \mathrm{v}$. $2,490.9$ Bcf), or by 4 percent. Table 4 on the next page compares the monthly volume and price data for natural gas imports from Canada during the first 9 months of 2000 and 1999. Most of the growth in imports this year are directly related to new gas flows being transported into the New England region by the new Maritimes system, as discussed above, and by increased utilization of the Portland Natural Gas Transmission System (PNGTS), which began operation in 1999. For the first 9 months of 2000, Maritimes shipped 82.7 Bcf and PNGTS increased its gas flows over its 1999 import level by $11.9 \mathrm{Bcf}$. Taken together, these two pipelines increased gas imports into New England by 94.6 Bcf; this represented 96 percent of the total incremental growth in Canadian gas imports so far this year.

For the $1^{\text {st }} 9$ months of 2000 , natural gas imports from Canada increased the most, both in terms of absolute volumes and \% of growth, in the U.S. Northeast. Besides the increase in volumes flowing into New England, as mentioned above, the Mid-Atlantic States, served by the Niagara Falls and Waddington entry points, also showed an aggregate year-to-year increase of $41.3 \mathrm{Bcf}$ (559.4 v. $518.1 \mathrm{Bcf}$ ), which represents an increase of $8 \%$. The only region of the country which experienced a drop in Canadian gas imports during the first 9 months of 2000 was the Pacific Northwest. Natural gas imports at the Sumas, WA., entry point declined by $58.1 \mathrm{Bcf}(252.6 \mathrm{v}$. $310.7 \mathrm{Bcf}$ ), or by 18.7 percent. Gas import volumes at the Eastport, ID., entry point, most of which were destined for the California market, experienced an increase of $29.3 \mathrm{Bcf}$ (613.3 v. 584 $\mathrm{Bcf})$, or a year-to-year increase of 5 percent. Imports at the major entry points transporting gas to the Midwest markets for the first 9 months of the year were virtually the same as the 1999 level; however, this will undoubtedly change with the recent start-up of the Alliance Pipeline on December 1.

The weighted average price of all Canadian natural gas imports at the international border during the first 9 months of 2000, as shown in Table 4 was $\$ 3.18$ per MMBtu, or an increase of $\$ 1.10$ per MMBtu over last year's average price $\$ 2.08$ during the same 9 month time period. This represents a year-to-year price increase of almost 53 percent.

Table 5 compares the volume of exports during the $1^{\text {st }} 9$ months of 2000 with 1999 . As shown, natural gas exports to Canada have grown by 24.1 Bcf this year, or by 97 percent. Similar to the price of natural gas imports, the price of gas exports also has risen substantially this year. The weighted average price of gas exported to Canada during the $1^{\text {st }} 9$ months of 2000 was $\$ 3.22$ per MMBtu, or an increase of $\$ 1.14$ per MMBtu over the same time period last year. This represents a year-to-year increase of nearly 55 percent. 
North American Gas Trade * North American Gas Trade * North American Gas Trade * North American Gas Trade * North American Gas Trade

Natural Gas Imports from Canada: First 9 Months (1999 \& 2000)

Table 4

\begin{tabular}{|c|c|c|c|c|c|c|c|c|c|c|c|}
\hline \multicolumn{10}{|c|}{ Volume: Billion Cubic Feet } \\
\hline & Jan. & Feb. & March & April & May & June & July & Aug. & Sept. & Total \\
\hline 1999 & 292.8 & 269.1 & 287.8 & 257.8 & 275.3 & 260.2 & 278.4 & 288.7 & 280.8 & 2490.9 \\
\hline 2000 & 310.2 & 289.2 & 291.4 & 273.9 & 274.6 & 278.5 & 293.4 & 295.5 & 282.9 & 2589.6 \\
\hline \multicolumn{10}{|c|}{ Price: \$/MMBtu } \\
\hline 1999 & 1.99 & 1.86 & 1.73 & 1.79 & 2.14 & 2.09 & 2.13 & 2.35 & 2.59 & $\$ 2.08$ \\
\hline 2000 & 2.38 & 2.52 & 2.55 & 2.80 & 3.00 & 3.82 & 3.91 & 3.58 & 4.11 & $\$ 3.18$ \\
\hline
\end{tabular}

Natural Gas Exports to Canada: First 9 Months (1999 \& 2000)

Table 5

\begin{tabular}{|l|c|c|c|c|c|c|c|c|c|c|c|}
\hline \multicolumn{10}{|c|}{ Volume: Billion Cubic Feet } \\
\hline & Jan. & Feb. & March & April & May & June & July & Aug. & Sept. & Total \\
\hline 1999 & 2.4 & 3.4 & 4.9 & 2.3 & 2.5 & 2.3 & 2.3 & 2.4 & 2.3 & 24.8 \\
\hline 2000 & 7.1 & 9.0 & 9.1 & 3.1 & 3.8 & 4.3 & 4.0 & 3.9 & 4.6 & 48.9 \\
\hline \multicolumn{10}{|c|}{ Price: \$/MMBtu } \\
\hline 1999 & 1.90 & 1.91 & 1.78 & 1.77 & 2.24 & 2.13 & 2.19 & 2.41 & 2.78 & $\$ 2.08$ \\
\hline 2000 & 2.44 & 2.65 & 2.69 & 2.81 & 3.09 & 4.11 & 4.29 & 3.83 & 4.68 & $\$ 3.22$ \\
\hline
\end{tabular}


Page - 1

\section{LONG-TERM IMPORTS}

\section{ACTUAL IMPORTS AS A PERCENT OF AUTHORIZED VOLUMES}

\section{IMPORTERIEXPORTER}

\section{ANDROSCOGGIN ENERGY LLCNarious Suppliers} AVISTA CORPORATION/AEC OIl \& Gas Company AVISTA CORPORATION/Engage Energy Canada AVISTA CORPORATION/Enron Capital \& Trade AVISTA CORPORATIONIPanCanadian Petroleum Limiled BAY STATE GAS COMPANY/Renaissance Energy Ltd. BEAR PAW ENERGY, L.L.C./Amoco Canada Resources Ltd. BOSTON GAS COMPANYIAlberta NE Gas (TCGS Ltd. 1) BOSTON GAS COMPANY/Imperial Oil Resources BOUNDARY GAS, INC./TransCanada Pipelines Limited BROOKLYN NAVY YARD COGEN. PARTNERS, L.P./Crestar Energy BROOKLYN NAVY YARD COGEN. PARTNERS, L.P./PanCanadian Petroleum Ltd. BROOKLYN UNION GAS COMPANYIAlberta NE Gas (AEC Oil \& Gas Company) BROOKLYN UNION GAS COMPANY/Alberta NE Gas (TCGS Ltd. 1) BROOKLYN UNION GAS COMPANYIAlberta NE Gas (TCGS Ltd. 2) BROOKLYN UNION GAS COMPANYIAlberta Northeast Gas (ProGas) BROOKLYN UNION GAS COMPANYIAlberta Northeast Gas (Producers Marketing) BROOKLYN UNION OF LONG ISLANDIAlberta NE Gas (AEC Oil \& Gas) BROOKLYN UNION OF LONG ISLAND/Alberta NE Gas (TCGS Ltd. 1) BROOKLYN UNION OF LONG ISLAND/Alberta NE Gas (TCGS Ltd. 2) BROOKLYN UNION OF LONG ISLAND/Alberta Northeast Gas (ProGas) BROOKLYN UNION OF LONG ISLAND/Alberta Northeast Gas (Producers Marketing) CASCADE NATURAL GAS COPORATION/IGI Resources, Inc. CASCADE NATURAL GAS CORPORATION/Duke Energy Trad. \& Mktg. Svcs., LLC CASCADE NATURAL GAS CORPORATION/Engage Energy Canada CASCADE NATURAL GAS CORPORATION/Engage Energy Canada CENTRAL HUDSON COMPANYIAlberta NE Gas (AEC Oil \& Gas Company) CENTRAL HUDSON COMPANYIAlberta NE Gas (TCGS Lid. 1) CENTRAL HUDSON COMPANYIAlberta NE Gas (TCGS Ltd. 2) CENTRAL HUDSON COMPANY/Alberta Northeast Gas (ProGas) CENTRAL HUDSON COMPANYIAlberta Northeast Gas (Producers Marketing) CHEVRON NATURAL GAS SERVICES, INC./Chevron Canada Resources CHEVRON U.S.A. INC./Chevron Canada Resources CHEVRON U.S.A. INC./Chevron Canada Resources CITY OF BURBANK, CALIFORNIAVEngage Energy Canada, L.P.

\begin{tabular}{|c|c|c|c|c|}
\hline $\begin{array}{c}1999 \\
\text { qtr } \\
\# 3\end{array}$ & $\begin{array}{c}1999 \\
\text { qtr } \\
\# 4\end{array}$ & 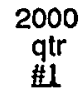 & $\begin{array}{c}2000 \\
\text { qtr } \\
\# 2\end{array}$ & $\begin{array}{c}2000 \\
\mathrm{qtr} \\
\sharp 3\end{array}$ \\
\hline N/A & 32 & 60 & 57 & 62 \\
\hline N/A & 73 & 88 & N/A & N/A \\
\hline 80 & 81 & 82 & 82 & 82 \\
\hline 96 & 75 & 103 & 103 & 103 \\
\hline 78 & 82 & 83 & 84 & 84 \\
\hline 100 & 100 & 100 & 100 & 100 \\
\hline 32 & 36 & 43 & 38 & 42 \\
\hline 99 & 87 & 98 & 99 & 94 \\
\hline N/A & N/A & 29 & 68 & 87 \\
\hline 100 & 98 & 80 & 99 & 100 \\
\hline 108 & 109 & 108 & 108 & 108 \\
\hline 108 & 109 & 108 & 108 & 108 \\
\hline 99 & 99 & 99 & 99 & 94 \\
\hline 100 & 100 & 100 & 100 & 95 \\
\hline 100 & 100 & 100 & 100 & 95 \\
\hline 100 & 84 & 100 & 100 & 100 \\
\hline 100 & 100 & 100 & 100 & 100 \\
\hline 99 & 81 & 99 & 99 & 99 \\
\hline 100 & 88 & 100 & 100 & 95 \\
\hline 100 & 88 & 100 & 100 & 95 \\
\hline 100 & 77 & 100 & 100 & 100 \\
\hline 99 & 86 & 99 & 99 & 99 \\
\hline N/A & 100 & 91 & N/A & N/A \\
\hline 88 & 89 & 90 & 98 & 97 \\
\hline 80 & 78 & 79 & 79 & 78 \\
\hline 49 & 71 & 73 & 22 & N/A \\
\hline 96 & 79 & 96 & 129 & 96 \\
\hline 100 & 88 & 100 & 100 & 95 \\
\hline 100 & 88 & 100 & 100 & 95 \\
\hline 102 & 122 & 102 & 102 & 102 \\
\hline 99 & 90 & 99 & 99 & 99 \\
\hline 79 & 81 & 80 & 78 & 83 \\
\hline 69 & 82 & 83 & 82 & 83 \\
\hline 105 & 96 & 109 & 105 & 101 \\
\hline 89 & 99 & N/A & N/A & N/A \\
\hline
\end{tabular}

\section{WEIGHTED AVERAGE PRICE (\$MMBTU) OFIMPORTS}

\begin{tabular}{|c|c|c|c|c|}
\hline $\begin{array}{c}1999 \\
\text { qtr } \\
\# 3\end{array}$ & $\begin{array}{c}1999 \\
\text { qtr } \\
114\end{array}$ & $\begin{array}{c}2000 \\
\text { qtr } \\
\mathbb{\# 1}\end{array}$ & $\begin{array}{c}2000 \\
\text { qtr } \\
\$ 2\end{array}$ & $\begin{array}{c}2000 \\
\text { qtr } \\
\# 3\end{array}$ \\
\hline N/A & 2.66 & 2.57 & 3.17 & 4.37 \\
\hline N/A & 2.45 & 2.36 & N/A & N/A \\
\hline 2.16 & 2.38 & 2.33 & 2.81 & 3.17 \\
\hline 2.16 & 2.34 & 2.49 & 2.92 & 3.61 \\
\hline 2.14 & 2.44 & 2.20 & 2.89 & 3.60 \\
\hline 3.03 & 3.36 & 3.03 & 3.56 & 4.98 \\
\hline 1.21 & 1.13 & 1.12 & 1.71 & 1.93 \\
\hline 2.79 & 2.73 & 2.68 & 3.61 & 4.48 \\
\hline N/A & $N / A$ & 2.93 & 3.82 & 4.59 \\
\hline 2.49 & 2.91 & 3.34 & 3.42 & 3.93 \\
\hline 3.07 & 3.09 & 3.05 & 3.95 & 4.80 \\
\hline 3.02 & 3.04 & 3.00 & 3.90 & 4.75 \\
\hline 2.80 & 2.98 & 3.53 & 4.15 & 4.65 \\
\hline 2.79 & 2.79 & 2.68 & 3.61 & 4.48 \\
\hline 2.79 & 2.79 & 2.68 & 3.61 & 4.48 \\
\hline 2.85 & 2.99 & 3.25 & 3.93 & 4.71 \\
\hline 2.85 & 3.05 & 3.26 & 3.59 & 4.71 \\
\hline 2.80 & 2.91 & 3.15 & 4.15 & 4.65 \\
\hline 2.79 & 2.73 & 2.68 & 3.61 & 4.48 \\
\hline 2.79 & 2.73 & 2.68 & 3.61 & 4.48 \\
\hline 2.85 & 2.96 & 3.25 & 3.93 & 4.71 \\
\hline 2.85 & 3.00 & 3.26 & 3.59 & 4.71 \\
\hline N/A & 2.77 & 2.85 & N/A & N/A \\
\hline 2.37 & 2.67 & 2.48 & 3.31 & 4.63 \\
\hline 2.07 & 2.52 & 2.75 & 2.59 & 3.19 \\
\hline 2.22 & 2.72 & 2.38 & 2.73 & $\mathrm{~N} / \mathrm{A}$ \\
\hline 2.80 & 2.91 & 3.53 & 4.12 & 4.65 \\
\hline 2.79 & 2.73 & 2.68 & 3.61 & 4.48 \\
\hline 2.79 & 2.73 & 2.68 & 3.61 & 4.48 \\
\hline 2.85 & 3.11 & 3.25 & 3,93 & 4.71 \\
\hline 2.85 & 3.01 & 3.26 & 3.59 & 4.71 \\
\hline 2.46 & 2.55 & 2.63 & 2.63 & 2.61 \\
\hline 1.99 & 2.26 & 2.14 & 2.89 & 3.87 \\
\hline 3.23 & 3.44 & 3.53 & 3.60 & 3.58 \\
\hline 1.93 & 1.91 & $N / A$ & $N / A$ & N/A \\
\hline
\end{tabular}


Page : 2

\section{LONG-TERM IMPORTS}

\section{ACTUALIMPORTS AS A PERCENT OE AUTHORIZED VOLUMES}

\section{IMPORTER/EXPORTER}

CITY OF DULUTH, MINNESOTA/ProGas Limited

CITY OF GLENDALE, CALIFORNIAVEngage Energy Canada, L.P.

CITY OF PASADENA, CALIFORNIAVEngage Energy Canada

COENERGY TRADING COMPANYNarious Suppliers

COLONIAL NATURAL GAS/Alberta NE Gas (TCGS Ltd. 1)

COMMONWEALTH GAS COMPANY/Alberta NE Gas (TCGS Ltd. 1)

CONNECTICUT NATURAL GAS/Alberta NE Gas (AEC Oil \& Gas Company)

CONNECTICUT NATURAL GASIAlberta NE Gas (TCGS Ltd. 1)

CONNECTICUT NATURAL GAS/Alberta NE Gas (TCGS Ltd. 2)

CONNECTICUT NATURAL GAS/Alberta Northeast Gas (ProGas)

CONNECTICUT NATURAL GAS/Alberta Northeast Gas (Producers Marketing)

CONSOLIDATED EDISON COMPANY OF N.Y./Alberta NE Gas (AEC Oil \& Gas)

CONSOLIDATED EDISON COMPANY OF N.Y./Alberta NE Gas (ProGas)

CONSOLIDATED EDISON COMPANY OF N.Y./Alberta NE Gas (Producers Marketing) 100

CONSOLIDATED EDISON COMPANY OF N.Y./Alberta NE Gas (TCGS Ltd. 1)

CONSOLIDATED EDISON COMPANY OF N.Y./Alberta NE Gas (TCGS Ltd. 2)

CONSOLIDATED EDISON COMPANY OF N.Y./Amoco Canada

CORAL ENERGY RESOURCES, L.P.-ENRON GAS MARKET/Shell Canada Limited

CORAL ENERGY RESOURCES, L.P.-MIDAMERICAN ENER/Shell Canada Limited

CRESTAR ENERGY MARKETING CORPORATION/Crestar Energy

CRESTAR ENERGY MARKETING CORPORATION/Creslar Energy

DARTMOUTH POWER ASSOCIATES, L.P./Anderson Exploration Lid.

DARTMOUTH POWER ASSOCIATES, L.P./Crestar Energy Inc.

DARTMOUTH POWER ASSOCIATES, L.P./Direct Energy Marketing Ltd.

DARTMOUTH POWER ASSOCIATES, L.P./Remington Energy Ltd.

DISTRIGAS CORPORATION (88-37-LNG)/Sonatrach

DISTRIGAS CORPORATION (89-16-LNG)/Sonatrach

DISTRIGAS CORPORATION (95-100-LNG)/Cabot LNG Trading Limited

DUKE ENERGY LNG SALES, INC./Sonatrading

ECOELECTRICA, L.P./Cabot LNG Trading Limited

ELIZABETHTOWN GAS COMPANY/TCGS Ltd.

ENCOGEN NORTHWEST, L.P./CanWest Gas Supply, lnc.

ENERGYNORTH, INC./Alberta NE Gas (TCGS Ltd. 1)

ENGAGE ENERGY CANADA, L.P. - CIBOLA CORP./Engage Energy Canada, L.P. 103

ENGAGE ENERGY CANADA, L.P. - I'STATE POWER CO/Engage Energy Canada, L.P.109

\begin{tabular}{|c|c|c|c|c|}
\hline $\begin{array}{c}1999 \\
\text { qtr } \\
\# 3\end{array}$ & $\begin{array}{c}1999 \\
\text { qtr } \\
\# 4\end{array}$ & $\begin{array}{c}2000 \\
\text { qtr } \\
\text { 柆 }\end{array}$ & 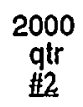 & $\begin{array}{c}2000 \\
\text { qtr } \\
\# 3\end{array}$ \\
\hline$N / A$ & 97 & 101 & 101 & 103 \\
\hline 99 & 101 & 99 & 98 & 99 \\
\hline 96 & 98 & 99 & 98 & 97 \\
\hline 31 & 55 & 75 & 10 & 1 \\
\hline 100 & 88 & 99 & 100 & 95 \\
\hline 100 & 88 & 99 & 100 & 95 \\
\hline 101 & 88 & 101 & 101 & 101 \\
\hline 100 & 88 & 100 & 100 & 98 \\
\hline 99 & 87 & 99 & 99 & 97 \\
\hline 100 & 78 & 100 & 100 & 98 \\
\hline 101 & 89 & 101 & 101 & 99 \\
\hline 100 & 83 & 101 & 101 & 101 \\
\hline 100 & 78 & 100 & 100 & 100 \\
\hline 100 & 91 & 100 & 100 & 100 \\
\hline 100 & 88 & 100 & 100 & 95 \\
\hline 100 & 88 & 100 & 100 & 95 \\
\hline 100 & 101 & 102 & 102 & 102 \\
\hline 101 & 101 & 101 & 103 & 101 \\
\hline 100 & 95 & 99 & 100 & 100 \\
\hline 79 & 88 & 96 & 98 & 87 \\
\hline 101 & 95 & 97 & 100 & 95 \\
\hline 117 & 72 & 129 & 123 & 105. \\
\hline 127 & 70 & 43 & 45 & 90 \\
\hline N/A & N/A & N/A & $N / A$ & N/A \\
\hline 80 & 64 & 64 & 61 & 46 \\
\hline N/A & N/A & N/A & N/A & N/A \\
\hline N/A & N/A & $N / A$ & N/A & N/A \\
\hline N/A & - N/A & N/A & N/A & N/A \\
\hline 63 & 65 & 60 & 59 & 59 \\
\hline N/A & N/A & N/A & N/A & N/A \\
\hline 100 & 100 & 100 & 100 & 100 \\
\hline 78 & 46 & 83 & 100 & 100 \\
\hline 100 & 91 & 99 & 100 & 95 \\
\hline 103 & 102 & 103 & 103 & 104 \\
\hline 109 & 110 & N/A & N/A & N/A \\
\hline
\end{tabular}

WEIGHTED AVERAGE PRICE (\$MMBTU) OE IMPORTS

$\begin{array}{ccccc}\begin{array}{c}1999 \\ \text { qtr }\end{array} & \begin{array}{c}1999 \\ \text { qtr }\end{array} & \begin{array}{c}2000 \\ \text { qtr }\end{array} & \begin{array}{c}2000 \\ \text { qtr }\end{array} & \begin{array}{c}2000 \\ \text { qtr }\end{array} \\ \text { \#3 } & \# 4 & \text { \#1 } & \text { \#2 } & \text { \#3 } \\ \text { N/A } & 2.61 & 2.25 & 3.00 & 3.69 \\ 1.91 & 1.91 & 1.95 & 1.95 & 2.74 \\ 1.89 & 1.90 & 1.93 & 1.91 & 2.69 \\ 2.71 & 2.75 & 3.45 & 3.30 & 5.26 \\ 2.79 & 2.73 & 2.68 & 3.61 & 4.48 \\ 2.79 & 2.73 & 2.68 & 3.61 & 4.48 \\ 2.80 & 2.93 & 3.15 & 4.15 & 4.65 \\ 2.79 & 2.73 & 2.68 & 3.61 & 4.49 \\ 2.79 & 2.73 & 2.68 & 3.61 & 4.49 \\ 2.85 & 2.96 & 3.25 & 3.93 & 4.71 \\ 2.85 & 3.01 & 3.26 & 3.59 & 4.71 \\ 2.81 & 2.91 & 3.15 & 4.15 & 4.65 \\ 2.85 & 2.96 & 3.25 & 3.93 & 4.71 \\ 2.85 & 3.01 & 3.26 & 3.59 & 4.60 \\ 2.79 & 2.73 & 2.68 & 3.61 & 4.48 \\ 2.79 & 2.73 & 2.68 & 3.61 & 4.48 \\ 2.68 & 3.03 & 2.69 & 3.22 & 4.60 \\ 2.09 & 2.26 & 2.13 & 2.77 & 3.57 \\ 2.09 & 2.22 & 2.19 & 3.09 & 3.57 \\ 2.87 & 2.86 & 2.82 & 3.73 & 4.68 \\ 2.66 & 2.65 & 2.58 & 3.42 & 4.29 \\ 2.84 & 3.19 & 3.16 & 3.68 & 5.00 \\ 3.62 & 3.58 & 3.24 & 3.95 & 4.50 \\ \text { N/A } & \text { N/A } & \text { N/A } & \text { N/A } & \text { N/A } \\ 3.66 & 3.71 & 3.08 & 3.32 & 4.74 \\ 2.36 & 2.61 & 3.03 & \text { N/A } & 3.44 \\ \text { N/A } & \text { N/A } & \text { N/A } & \text { N/A } & 3.28 \\ 2.24 & 2.73 & 2.80 & 2.94 & 2.94 \\ 2.00 & 2.00 & 2.00 & 2.78 & 3.34 \\ \text { N/A } & \text { N/A } & \text { N/A } & \text { N/A } & 3.14 \\ 3.01 & 3.12 & 3.12 & 3.79 & 4.18 \\ 2.35 & 2.46 & 2.47 & 2.47 & 2.55 \\ 2.79 & 2.75 & 2.68 & 3.61 & 4.48 \\ 2.33 & 2.39 & 2.33 & 3.18 & 4.00 \\ 2.33 & 2.35 & \text { N/A } & \text { N/A } & \text { N/A }\end{array}$




\section{LONG-TERM IMPORTS}

\section{ACTUALIMPORTS AS A PERCENT OF AUTHORIZED VOLUMES}

\begin{tabular}{|c|c|c|c|c|}
\hline $\begin{array}{c}1999 \\
\text { qtr } \\
\# 3\end{array}$ & 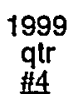 & $\begin{array}{c}2000 \\
\text { qtr } \\
\# 1\end{array}$ & $\begin{array}{c}2000 \\
\text { qtr } \\
\$ 2\end{array}$ & $\begin{array}{c}2000 \\
\text { qtr } \\
\sharp 3\end{array}$ \\
\hline
\end{tabular}

ENGAGE ENERGY CANADA, L.P. - METRO. UTIL. DIS/Engage Energy Canada, L.P. 102 ENGAGE ENERGY CANADA, L.P. - MIDAMERICAN/Engage Energy Canada, L.P. 103 ENGAGE ENERGY CANADA, L.P. - N'WESTERN PUB./Engage Energy Canada, L.P. 107 ENGAGE ENERGY CANADA, L.P. - WISCONSIN GAS/Engage Energy Canada, L.P. 103 ENGAGE ENERGY U.S., L.P./Engage Energy Canada, L.P. ENGAGE ENERGY U.S., L.P.Morgan Hydrocarbons Inc. ENGAGE ENERGY U.S., L.P.Morrison Petroleums Ltd. ENGAGE ENERGY U.S., L.P./Petro-Canada Resources ENGAGE ENERGY U.S., L.P./Rio Alto, Pinnacle, Jordan ENGAGE ENERGY U.S., L.P.Narious Suppliers ENGAGE ENERGY U.S., L.P.Narious Suppliers ENGAGE ENERGY U.S., L.P.Narious Suppliers ENRON NORTH AMERICA CORP./ECTR - Canada ENRON NORTH AMERICA CORP.IECTR - Canada ENRON NORTH AMERICA CORP./ECTR - Canada ENRON NORTH AMERICA CORP./ECTR - Canada ENRON NORTH AMERICA CORP./ECTR - Canada ESSEX COUNTY GAS CO./Alberta NE Gas (TCGS Lid. 1) GRANITE STATE GAS TRANSMISSION, INC./Direct Energy Marketing Ltd. GRANITE STATE GAS TRANSMISSION, INC./Shell Canada Limited HERMISTON GENERATING COMPANY, L.P./Home Oil Company Limited HERMISTON GENERATING COMPANY, L.P.TTransCanada Gas Services Lid. HESS ENERGY INC./Talisman Energy Inc. HESS ENERGY INC./Talisman Energy Inc. HUSKY GAS MARKETING INC./Husky Oil Operations Ltd. IGI RESOURCES, INC.MObil Oil Canada

LOCKPORT ENERGY ASSOCIATES, L.P./ProGas Limited MIDLAND COGENERATION VENTURE1/Anadarko Trading Company MIDLAND COGENERATION VENTURE2/Coral Energy MIDLAND COGENERATION VENTUREB/Husky Oil

MIDLAND COGENERATION VENTURE4/TransCanada Pipelines Limited MIDLAND COGENERATION VENTURE5/Burtington Resources Canada Energy Ltd. MIDLAND COGENERATION VENTURE6/Anadarko Trading Company MINNEGASCO, INC./TransCanada Pipelines Limited N.Y. STATE ELECTRIC \& GAS CO./Alberta NE Gas (AEC Oil \& Gas Co.)

$\begin{array}{rrrrr}102 & 74 & 103 & 102 & 102 \\ 103 & 82 & 103 & 102 & 102 \\ 107 & 83 & 108 & 106 & 101 \\ 103 & 104 & 104 & 103 & 103 \\ 21 & 17 & \text { N/A } & \text { N/A } & \text { N/A } \\ 102 & 102 & 99 & 101 & 102 \\ 102 & 102 & 103 & 102 & 103 \\ 101 & 101 & 101 & 101 & 101 \\ 65 & 65 & 66 & 65 & 64 \\ 61 & 61 & 61 & 61 & 61 \\ 90 & 96 & \text { N/A } & \text { N/A } & \text { N/A } \\ 98 & 100 & 69 & 98 & 97 \\ 13 & 13 & 13 & 13 & 13 \\ 101 & 101 & 101 & 101 & 101 \\ 95 & 95 & 96 & 95 & 95 \\ 104 & 104 & 104 & 97 & 87 \\ \text { N/A } & 101 & 101 & 101 & 101 \\ 100 & 88 & 99 & 100 & 95 \\ 100 & 100 & 101 & 100 & 100 \\ 101 & 101 & 101 & 101 & 101 \\ 72 & 72 & 73 & 86 & 92 \\ 88 & 90 & 88 & 86 & 92 \\ 93 & 91 & 96 & 86 & 91 \\ 93 & 93 & 96 & 91 & 86 \\ 84 & 85 & 59 & 96 & 94 \\ \text { N/A } & \text { N/A } & \text { N/A } & 0 & \text { N/A } \\ 87 & 60 & 68 & 30 & 81 \\ 100 & 100 & 100 & 100 & 100 \\ 100 & 100 & 100 & 100 & 100 \\ 100 & 100 & 100 & 100 & 100 \\ 100 & 100 & 100 & 100 & 100 \\ 100 & 100 & 100 & 100 & 100 \\ \text { N/A } & 100 & 100 & 101 & 101 \\ 62 & 62 & 62 & 62 & 62 \\ 102 & 84 & 102 & 102 & 97\end{array}$

WEIGHTED AVERAGE PRICE (\$MMBTU) OFIMPORTS

$\begin{array}{ccccc}1999 & 1999 & 2000 & 2000 & 2000 \\ \text { qtr } & \begin{array}{c}\text { qtr } \\ \text { qt }\end{array} & \begin{array}{c}\text { qtr } \\ \text { qt1 }\end{array} & \begin{array}{c}\text { qtr } \\ \text { \#13 }\end{array} \\ 2.46 & 2.20 & 2.36 & 3.22 & 4.00 \\ 2.28 & 2.14 & 2.22 & 3.00 & 3.65 \\ 2.43 & 2.24 & 2.36 & 3.22 & 4.01 \\ 2.36 & 2.29 & 2.28 & 3.11 & 3.88 \\ 2.50 & 2.95 & \text { N/A } & \text { N/A } & \text { N/A } \\ 2.77 & 2.92 & 2.97 & 3.73 & 4.58 \\ 2.33 & 2.13 & 2.12 & 2.97 & 3.70 \\ 2.33 & 2.13 & 2.12 & 2.93 & 3.66 \\ 2.77 & 2.92 & 2.93 & 3.73 & 4.57 \\ 2.68 & 2.74 & 2.57 & 3.59 & 4.38 \\ 2.66 & 2.93 & \text { N/A } & \text { N/A } & \text { N/A } \\ 2.56 & 2.62 & 2.50 & 3.55 & 4.36 \\ 2.47 & 2.48 & 2.44 & 3.36 & 4.18 \\ 2.67 & 2.73 & 2.64 & 3.67 & 4.47 \\ 2.54 & 2.59 & 2.24 & 3.23 & 4.10 \\ 2.67 & 2.69 & 2.52 & 3.53 & 4.39 \\ \text { N/A } & 2.74 & 2.56 & 3.19 & 3.85 \\ 2.79 & 2.73 & 2.68 & 3.61 & 4.48 \\ 2.49 & 2.34 & 2.15 & 2.17 & 4.48 \\ 2.60 & 2.86 & 3.16 & 3.66 & 4.39 \\ 2.48 & 2.56 & 2.65 & 2.63 & 2.62 \\ 2.48 & 2.56 & 2.65 & 2.63 & 2.62 \\ 1.89 & 1.97 & 2.11 & 2.07 & 2.24 \\ 1.90 & 2.01 & 2.11 & 2.09 & 2.45 \\ 2.15 & 3.04 & 3.41 & 2.67 & 5.60 \\ \text { N/A } & \text { N/A } & \text { N/A } & \text { N/A } & \text { N/A } \\ 3.93 & 4.66 & 4.63 & 6.73 & 4.35 \\ 1.66 & 1.65 & 1.64 & 1.65 & 1.65 \\ 1.58 & 1.57 & 1.70 & 1.55 & 1.54 \\ 1.90 & 1.90 & 1.90 & 1.90 & 1.90 \\ 1.57 & 1.56 & 1.56 & 1.56 & 1.56 \\ 2.49 & 2.52 & 2.55 & 2.58 & 2.61 \\ \text { N/A } & 2.99 & 3.02 & 3.04 & 3.07 \\ 2.37 & 2.40 & 2.44 & 3.23 & 4.11 \\ 2.80 & 2.91 & 3.15 & 4.15 & 4.65\end{array}$




\section{LONG-TERM IMPORTS}

\section{ACTUAL IMPORTS AS A PERCENT OF AUTHORIZED VOLUMES}

N.Y. STATE ELECTRIC \& GAS CO./Alberta NE Gas (TCGS Ltd. 2) N.Y. STATE ELECTRIC \& GAS CO./Aiberta Northeast Gas (ProGas) N.Y. STATE ELECTRIC \& GAS CO./Alberta Northeast Gas (Producers Marketing) N.Y. STATE ELECTRIC \& GAS CO./Crestar Energy N.Y. STATE ELECTRIC \& GAS CO./Progas Limited NATIONAL FUEL GAS DISTRIBUTION CORP./Alberta NE Gas (TCGS Ltd.) NATIONAL STEEL CORPORATION/Direct Energy Marketing, Ltd. NEW JEFSEY NATURAL GAS COMPANY/Alberta NE Gas (AEC Oil \& Gas Company) 10 NEW JERSEY NATURAL GAS COMPANY/Alberta NE Gas (TCGS Ltd. 1) NEW JERSEY NATURAL GAS COMPANY/Alberta NE Gas (TCGS Ltd. 2) NEW JERSEY NATURAL GAS COMPANY/Alberta Northeast Gas (ProGas) NEW JERSEY NATURAL GAS COMPANY/Alberta Northeast Gas (Producers Mktg.) NIAGARA MOHAWK POWER CORPORATION/TCGS Ltd. NORTH JERSEY ENERGY ASSOCIATES/ProGas Limited NORTHEAST ENERGY ASSOCIATES/ProGas Limited NORTHERN MINNESOTA UTILITIES/TCGS Ltd. NORTHERN MINNESOTA UTILITIES - EASTERN MARKET/TCGS LId. NORTHERN MINNESOTA UTILITIES - WESTERN MARKET/TCGS Ltd. NORTHERN STATES POWER COMPANY/Amoco Canada (WI) NORTHERN STATES POWER COMPANY/Canadian Occidental (WI) NORTHERN STATES POWER COMPANY/ProGas Limiled (WI) NORTHERN STATES POWER COMPANY (MINNESOTA)/Amoco Canada (MN) NORTHERN STATES POWER COMPANY (MINNESOTA)/TCGS LId. NORTHERN UTILITIES, INC./Renaissance Energy Ltd. NORTHWEST NATURAL GAS COMPANY/Amoco Canada NORTHWEST NATURAL GAS COMPANY/Burlington Resources Canada Energy Ltd. NORTHWEST NATURAL GAS COMPANY/CanWest Gas Supply NORTHWEST NATURAL GAS COMPANYIEngage Energy Canada NORTHWEST NATURAL GAS COMPANY/SUmmit Resources Limited NUI CORPORATION/TCGS LId.

NW ALASKAN PIPELINE CO-Pan-Alberta Gas (I)/Pan-Alberta Gas Ltd. NW ALASKAN PIPELINE CO-Pan-Alberta Gas (II)/Pan-Alberta Gas Lid. OCEAN STATE POWER/ProGas Limited

OCEAN STATE POWER II/ProGas Limited OCEAN STATE POWER II/TCGS LId.

\begin{tabular}{|c|c|c|c|c|}
\hline $\begin{array}{c}1999 \\
\text { qtr } \\
\mathbb{1 3}\end{array}$ & $\begin{array}{c}1999 \\
\text { qtr } \\
\mathbb{\# 4}\end{array}$ & $\begin{array}{c}2000 \\
\text { qtr } \\
\text { 訨 }\end{array}$ & 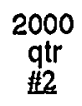 & $\begin{array}{r}2000 \\
\text { qtr } \\
\text { \&3 }\end{array}$ \\
\hline 99 & 87 & 99 & 99 & 94 \\
\hline 101 & 79 & 101 & 101 & 95 \\
\hline 99 & 90 & 99 & 99 & 94 \\
\hline 38 & 79 & 87 & 94 & 72 \\
\hline 90 & 86 & 89 & 89 & 89 \\
\hline 100 & 91 & 99 & 100 & 100 \\
\hline 95 & 128 & 125 & 91 & 101 \\
\hline 102 & 90 & 102 & 102 & 101 \\
\hline 100 & 88 & 100 & 100 & 99 \\
\hline 99 & 87 & 99 & 99 & 97 \\
\hline 100 & 81 & 100 & 100 & 99 \\
\hline 99 & 87 & 99 & 99 & 98 \\
\hline 100 & 101 & 100 & 100 & 100 \\
\hline 90 & 90 & 90 & 91 & 93 \\
\hline 97 & 100 & 100 & 96 & 103 \\
\hline 100 & 100 & 100 & 100 & 100 \\
\hline 3 & 7 & 10 & 3 & 2 \\
\hline 24 & 83 & 126 & 29 & 18 \\
\hline 40 & 61 & 86 & 97 & 74 \\
\hline 99 & 112 & 99 & 100 & 65 \\
\hline 97 & 94 & 99 & 100 & 63 \\
\hline 99 & 94 & 100 & 100 & 99 \\
\hline 100 & 91 & 142 & 100 & 96 \\
\hline 99 & 99 & 99 & 99 & 99 \\
\hline 103 & 103 & 102 & 102 & 102 \\
\hline 69 & 99 & 98 & 70 & 71 \\
\hline 102 & 103 & 102 & 102 & 102 \\
\hline 59 & 59 & 59 & 59 & 59 \\
\hline 63 & 98 & 98 & 62 & 63 \\
\hline 100 & 84 & 100 & 100 & 100 \\
\hline 101 & 94 & 102 & 101 & 100 \\
\hline 80 & 78 & 81 & 80 & 80 \\
\hline 100 & 100 & 100 & 100 & 100 \\
\hline 100 & 100 & 100 & 100 & 100 \\
\hline 100 & 100 & 100 & 100 & 100 \\
\hline
\end{tabular}

WEIGHTED AVERAGE PRICE (\$MMBTU) OF IMPORTS

\begin{tabular}{|c|c|c|c|c|}
\hline $\begin{array}{c}1999 \\
\text { qtr } \\
\mathbb{1 3}\end{array}$ & $\begin{array}{c}1999 \\
\text { gtr } \\
\# 4\end{array}$ & $\begin{array}{c}2000 \\
\text { qtr } \\
\# 1\end{array}$ & $\begin{array}{c}2000 \\
\text { qtr } \\
\# 2\end{array}$ & $\begin{array}{c}2000 \\
\text { qtr } \\
\$ 3\end{array}$ \\
\hline 2.79 & 2.73 & 2.68 & 3.61 & 4.48 \\
\hline 2.85 & 2.96 & 3.25 & 3.93 & 4.71 \\
\hline 2.85 & 3.01 & 3.26 & 3.59 & $\cdot 4.71$ \\
\hline 4.98 & 3.90 & 3.83 & 4.51 & 5.49 \\
\hline 2.79 & 3.20 & 3.59 & 3.67 & 3.79 \\
\hline 2.79 & 2.74 & 2.68 & 3.61 & 4.49 \\
\hline 2.38 & 2.51 & 2.90 & 3.04 & 3.60 \\
\hline 2.80 & 2.93 & 3.15 & 4.15 & 4.65 \\
\hline 2.79 & 2.73 & 2.68 & 3.61 & 4.49 \\
\hline 2.79 & 2.73 & 2.68 & 3.61 & 4.49 \\
\hline 2.85 & 2.97 & 3.25 & 3.93 & 4.71 \\
\hline 2.85 & 3.01 & 3.26 & 3,59 & 4.71 \\
\hline 3.05 & 2.98 & 2.94 & 3.88 & 4.72 \\
\hline 2.97 & 2.97 & 3.34 & 3.33 & 3.20 \\
\hline 3.63 & 3.60 & 3.80 & 3.83 & 3.74 \\
\hline 2.32 & 2.33 & 2.21 & 3.30 & 3.89 \\
\hline 2.38 & 2.46 & 2.46 & 3.18 & 4.10 \\
\hline 2.44 & 2.50 & 2.46 & 3.19 & 4.23 \\
\hline 2.78 & 2.37 & 2.56 & 2.59 & 2.51 \\
\hline 3.01 & 3.18 & 2.83 & 3.59 & 4.70 \\
\hline 2.80 & 2.88 & 2.89 & 3.68 & 4.79 \\
\hline 2.76 & 2.74 & 2.58 & 3.37 & 4.08 \\
\hline 2.65 & 2.73 & 2.71 & 3.57 & 4.57 \\
\hline 3.03 & 3.34 & 3.03 & 3.57 & 4.98 \\
\hline 1.80 & 2.47 & 2.46 & 2.46 & 2.46 \\
\hline 2.22 & 2.57 & 2.63 & 2.69 & 2.42 \\
\hline 2.25 & 2.56 & 2.36 & 3.08 & 3.59 \\
\hline 2.22 & 2.64 & 2.64 & 2.89 & 3.26 \\
\hline 2.04 & 2.61 & 2.71 & 2.48 & 2.47 \\
\hline 3.19 & 3.17 & 3.18 & 4.09 & 4.85 \\
\hline 2.30 & 2.38 & 2.29 & 3.06 & 3.94 \\
\hline 2.07 & 2.16 & 2.08 & 2.85 & 3.68 \\
\hline 1.80 & 1.80 & 2.23 & 2.60 & 3.25 \\
\hline 1.80 & 1.80 & 2.23 & 2.60 & 3.25 \\
\hline 1.80 & 1.81 & 2.23 & 2.60 & 3.25 \\
\hline
\end{tabular}


LONG-TERM IMPORTS

\section{ACTUAL IMPORTS AS A PERCENT OF AUTHORIZED VOLUMES}

\begin{tabular}{|c|c|c|c|c|}
\hline 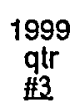 & 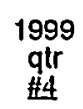 & $\begin{array}{c}2000 \\
\text { qtr } \\
\# 1\end{array}$ & 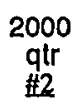 & $\begin{array}{c}2000 \\
\text { qtr } \\
\# 3\end{array}$ \\
\hline 100 & 100 & 101 & 101 & 101 \\
\hline 100 & 100 & 100 & 100 & 100 \\
\hline 90 & 99 & 92 & 73 & 74 \\
\hline 82 & 84 & 76 & 88 & 91 \\
\hline 83 & 81 & 73 & 85 & 88 \\
\hline 99 & 101 & 100 & 100 & 81 \\
\hline 100 & 102 & 100 & 100 & 90 \\
\hline 99 & 99 & N/A & N/A & N/A \\
\hline 0 & 2 & 2 & 0 & 0 \\
\hline $100^{\circ}$ & 100 & 100 & 102 & 100 \\
\hline 72 & 98 & 99 & 90 & 73 \\
\hline 50 & 97 & 102 & 55 & 44 \\
\hline 56 & 56 & 56 & 56 & 56 \\
\hline 81 & 81 & 81 & 81 & 81 \\
\hline 97 & 98 & 97 & 97 & 96 \\
\hline 97 & 78 & 97 & 96 & 97 \\
\hline 100 & 99 & 103 & 98 & 92 \\
\hline 89 & 92 & 92 & 92 & 91 \\
\hline N/A & N/A & N/A & N/A & N/A \\
\hline 100 & 93 & 99 & 100 & 95 \\
\hline 104 & 100 & 85 & 96 & 104 \\
\hline 103 & 100 & 103 & 103 & 103 \\
\hline 104 & 119 & 186 & 196 & 208 \\
\hline 81 & 77 & 81 & 81 & 81 \\
\hline 104 & 102 & 104 & 104 & 103 \\
\hline N/A & N/A & N/A & 100 & 88 \\
\hline 99 & 102 & 103 & 103 & 102 \\
\hline 100 & 100 & 99 & 100 & 99 \\
\hline N/A & 61 & 60 & N/A & N/A \\
\hline 85 & 97 & 90 & 74 & 95 \\
\hline 85 & 83 & 88 & 83 & 87 \\
\hline 100 & 100 & 100 & 100 & 99 \\
\hline 100 & 100 & 100 & 100 & 99 \\
\hline 92 & 90 & 92 & 92 & 91 \\
\hline 99 & 99 & 99 & 100 & 98 \\
\hline
\end{tabular}

WEIGHTED AVERAGE PRICE (\$MMBTU) OF IMPORTS

$\begin{array}{ccccc}\begin{array}{c}1999 \\ \text { qtr }\end{array} & \begin{array}{c}1999 \\ \text { qtr }\end{array} & \begin{array}{c}2000 \\ \text { qtr }\end{array} & \begin{array}{c}2000 \\ \text { qtr }\end{array} & \begin{array}{c}2000 \\ \text { qtr }\end{array} \\ \text { \#3 } & \# 4 & \# 1 & \mathbb{\# 2} & \# 3 \\ 3.29 & 3.47 & 3.48 & 4.24 & 5.01 \\ 2.68 & 3.27 & 3.27 & 3.50 & 3.52 \\ 1.73 & 1.97 & 1.82 & 2.01 & 3.11 \\ 2.96 & 2.93 & 3.17 & 3.44 & 3.71 \\ 3.13 & 3.19 & 3.51 & 3.72 & 3.80 \\ 2.59 & 2.72 & 3.03 & 3.12 & 3.32 \\ 3.20 & 2.96 & 3.18 & 3.58 & 3.95 \\ 2.27 & 2.44 & \text { N/A } & \text { N/A } & \text { N/A } \\ 3.13 & 2.76 & 2.94 & 3.40 & 3.45 \\ 2.22 & 2.44 & 2.52 & 2.26 & 2.25 \\ 2.49 & 2.51 & 2.42 & 3.25 & 4.07 \\ 2.89 & 2.87 & 2.86 & 3.74 & 4.85 \\ 2.81 & 2.79 & 2.67 & 3.60 & 4.48 \\ 2.52 & 2.58 & 2.52 & 3.37 & 4.19 \\ 2.41 & 2.42 & 2.34 & 3.24 & 4.08 \\ 2.41 & 2.42 & 2.34 & 3.24 & 4.07 \\ 2.71 & 2.90 & 3.25 & 3.77 & 4.57 \\ 2.35 & 2.70 & 2.58 & 3.45 & 4.56 \\ 3.04 & 2.97 & 2.63 & 3.12 & 3.97 \\ 2.79 & 2.77 & 2.68 & 3.61 & 4.48 \\ 2.26 & 2.60 & 2.31 & 3.02 & 3.55 \\ 2.26 & 2.60 & 2.34 & 3.07 & 3.52 \\ 2.33 & 2.78 & 2.38 & 3.08 & 3.53 \\ 2.21 & 2.50 & 2.39 & 2.83 & 3.25 \\ 2.26 & 2.56 & 2.41 & 3.12 & 3.54 \\ \text { N/A } & \text { N/A } & \text { N/A } & 3.03 & 3.57 \\ 2.02 & 2.28 & 2.17 & 3.04 & 3.91 \\ 3.10 & 3.36 & 3.15 & 4.13 & 4.07 \\ \text { N/A } & 2.74 & 3.75 & \text { N/A } & \text { N/A } \\ 4.49 & 3.33 & 3.17 & 3.86 & 4.57 \\ 3.61 & 3.71 & 3.76 & 3.76 & 3.76 \\ 2.46 & 2.61 & 2.96 & 3.36 & 3.50 \\ 2.47 & 2.61 & 2.99 & 3.40 & 3.54 \\ 3.05 & 3.32 & 3.09 & 4.00 & 4.60 \\ 2.39 & 2.54 & 2.88 & 3.26 & 3.40\end{array}$

ORANGE AND ROCKLAND UTILITIES, INC./Wascana Energy Marketing ORCHARD GAS CORPORATION/ProGas Limited

PAN-ALBERTA GAS (U.S.) INC./Pan-Alberta Gas Ltd.

PAWTUCKET POWER ASSOCIATES/Anderson Exploration Lid.

PAWTUCKET POWER ASSOCIATES/Tarragon Oil \& Gas Limited

PITTSFIELD GENERATING CO., L.P.IAnderson Exploration Ltd.

PITTSFIELD GENERATING CO., L.P./Talisman Energy Inc.

POCO MARKETING LTD./IGI Resources, inc.

PORTAL MUNICIPAL GAS/Sask Energy Inc.

PROGAS U.S.A. INC.IProGas Limited

PROGAS U.S.A. INC.IProGas Limited

PROGAS U.S.A. INC./ProGas Limited

PROGAS U.S.A. INC./ProGas Limiled

PROGAS U.S.A. INC.IProGas Limited

PROGAS U.S.A. INC. - (DYNEGY)/ProGas LId.

PROGAS U.S.A. INC. - (TENASKA GAS COMPANY)/ProGas Limited

PROGAS U.S.A., INC/Progas U.S.A. Inc.

PROGAS U.S.A., INC/Progas U.S.A. Inc.

PROJECT ORANGE ASSOCIATES, L.P./Noranda, Inc.

PUBLIC SERVICE ELECTRIC \& GASIAlberta NE Gas (TCGS Lid. 1)

PUGET SOUND ENERGY, INC.IAmoco Canada

PUGET SOUND ENERGY, INC./Duke Energy

PUGET SOUND ENERGY, INC./Engage Energy Canada

PUGET SOUND ENERGY, INC./Engage Energy Canada

PUGET SOUND ENERGY, INC./Poco Petroleums Lid.

RDO FOODS CO./ProGas Limited

RENAISSANCE ENERGY (U.S.) INC./Renaissance Energy Limiled RENAISSANCE ENERGY (U.S.) INC./Renaissance Energy Limited RENAISSANCE ENERGY (U.S.) INC./Renaissance Energy Limited ROCK-TENN COMPANY, MILL DIV., INC.Mascana Energy Inc. SARANAC POWER PARTNERS, L.P./Shell Canada Limited SELKIRK COGEN PARTNERS, L.P./Imperial Oil Resources Limited SELKIRK COGEN PARTNERS, L.P.IPanCanadian Petroleum Lid. SELKIRK COGEN PARTNERS, L.P./Paramount Resources Ltd. SELKIRK COGEN PARTNERS, L.P./Producers Marketing, Ltd.

arketing




\section{LONG-TERM IMPORTS}

\section{ACTUALIMPORTS AS A PERCENT OF AUTHORIZED VOLUMES}

\section{IMPORTER/EXPORTER}

SIERRA PACIFIC POWER COMPANY/AMOco Canada

SITHE/INDEPENDENCE POWER PARTNERS, L.P./Enron Power Services Inc. SOUTHEASTERN MICHIGAN GAS COMPANYITCGS LId.

SOUTHERN CONNECTICUT GAS/Alberta NE Gas (AEC Oil \& Gas Company) SOUTHERN CONNECTICUT GAS/Aiberta NE Gas (TCGS Ltd. 1) SOUTHERN CONNECTICUT GAS/AIberta NE Gas (TCGS Ltd. 2) SOUTHERN CONNECTICUT GAS/Alberta Northeast Gas (ProGas) SOUTHERN CONNECTICUT GAS/Alberta Northeast Gas (Producers Marketing) ST. LAWRENCE GAS COMPANY, INC./TCGS Ltd. SUMAS COGENERATION COMPANY, L.P.IENCO Gas, Ltd. TENASKA WASHINGTON PARTNERS, L.P./Puget Sound Energy, Inc. THE U.S. GENERAL SERVICES ADMIN./Chief Mountain Natural Gas Coop. TM STAR FUEL COMPANY/CanWest Gas Supply

TRANSCANADA GAS SERVICES INC./TransCanada Gas Services TRANSCANADA GAS SERVICES INC./Yankee Gas Services TRANSCO ENERGY MARKETING COMPANY/Canstates Gas Marketing UNITED STATES GYPSUM COMPANY/Husky Oil Operations Lid. UNITED STATES GYPSUM COMPANY/Renaissance Energy, Ltd. UTILICORP UNITED, INC./ProGas Limited

UTILICORP UNITED, INC./TCGS Ltd.

VALLEY GAS COMPANYIAlberta NE Gas (TCGS Ltd. 1)

VERMONT GAS SYSTEMS INC./TCGS LId.

VERMONT GAS SYSTEMS, INC./Renaissance Energy Lid.

VERMONT GAS SYSTEMS, INC.Narious Suppliers

WISCONSIN ELECTRIC POWER CO. (GAS OPS)/Progas Limited

WISCONSIN ELECTRIC POWER CO. (GAS OPS)/TCGS LId.

WISCONSIN FUEL \& LIGHT COMPANYIProgas Limited

WISCONSIN FUEL \& LIGHT COMPANYTTCGS LId.

WISCONSIN GAS COMPANYIProGas Limited (I)

WISCONSIN GAS COMPANY/ProGas Limited (II)

WISCONSIN GAS COMPANYITCGS LId.

WISCONSIN GAS COMPANYITCGS LId.

WISCONSIN POWER \& LIGHT COMPANYIProGas Limited

WISCONSIN POWER \& LIGHT COMPANYITCGS Ltd.

WISCONSIN PUBLIC SERVICE CORPORATION/ProGas Limited

\begin{tabular}{|c|c|c|c|c|}
\hline $\begin{array}{c}1999 \\
\text { qtr } \\
\# 3\end{array}$ & 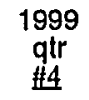 & $\begin{array}{c}2000 \\
\text { qtr } \\
\# 1\end{array}$ & $\begin{array}{c}2000 \\
q t r \\
\# 2\end{array}$ & $\begin{array}{c}2000 \\
\text { qtr } \\
\# 3\end{array}$ \\
\hline 99 & 98 & 99 & N/A & N/A \\
\hline 86 & 88 & 88 & 88 & 88 \\
\hline 101 & 100 & 100 & 100 & 100 \\
\hline 99 & 81 & 99 & 99 & 99 \\
\hline 150 & 138 & 150 & 150 & 142 \\
\hline$N / A$ & N/A & N/A & 0 & N/A \\
\hline 100 & 80 & 100 & 100 & 28 \\
\hline 101 & 91 & 101 & 101 & 178 \\
\hline 13 & 60 & 72 & 34 & 12 \\
\hline 96 & 99 & 102 & 83 & 97 \\
\hline 0 & N/A & N/A & N/A & N/A \\
\hline 0 & 1 & 1 & 1 & 0 \\
\hline 90 & 92 & 97 & 100 & 100 \\
\hline 44 & 26 & 13 & 13 & 12 \\
\hline N/A & 72 & 72 & 72 & 72 \\
\hline 107. & 79 & 101 & 101 & 91 \\
\hline 122 & 122 & 121 & 122 & 122 \\
\hline 97 & 100 & 99 & 95 & 97 \\
\hline 95 & 96 & 96 & 94 & 95 \\
\hline 100 & 100 & 100 & 98 & 100 \\
\hline 100 & 100 & 99 & 100 & 95 \\
\hline 63 & 88 & 101 & 68 & 28 \\
\hline 38 & 100 & 100 & 79 & 79 \\
\hline$N / A$ & 21 & 55 & 21 & N/A \\
\hline 99 & 80 & 100 & 99 & 100 \\
\hline 99 & 83 & 91 & 95 & 99 \\
\hline 99 & 100 & 100 & 99 & 100 \\
\hline 101 & 83 & 100 & 97 & 86 \\
\hline 77 & 76 & 101 & 98 & 78 \\
\hline 78 & 75 & 101 & 98 & 79 \\
\hline 77 & 65 & 92 & 99 & 86 \\
\hline 82 & 510 & 102 & 101 & 76 \\
\hline 99 & 99 & 99 & 99 & 99 \\
\hline 88 & 77 & 99 & 99 & 94 \\
\hline 84 & 100 & 100 & 98 & 100 \\
\hline
\end{tabular}

WEIGHTED AVERAGE PRICE (\$MMBTU) OFIMPORTS

\begin{tabular}{|c|c|c|c|c|}
\hline $\begin{array}{c}1999 \\
\text { qtr } \\
\mathbb{1 3}\end{array}$ & $\begin{array}{c}1999 \\
\text { qtr } \\
\# 4\end{array}$ & $\begin{array}{c}2000 \\
\text { qtr } \\
\mathbb{\# 1}\end{array}$ & $\begin{array}{c}2000 \\
\text { qtr } \\
\# 2\end{array}$ & 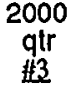 \\
\hline 1.34 & 1.34 & 1.34 & N/A & N/A \\
\hline 4,16 & 4.16 & 2.95 & 3.58 & 3.69 \\
\hline 2,43 & 2.44 & 2.32 & 3.26 & 4.14 \\
\hline 2.80 & 2.91 & 3.15 & 4.15 & 4.65 \\
\hline 2.79 & 2.76 & 2.68 & 3.61 & 4.48 \\
\hline N/A & N/A & $N / A$ & N/A & N/A \\
\hline 2.85 & 2.97 & 3.25 & 3.93 & 4.61 \\
\hline 2,85 & 3.01 & 3.26 & 3.59 & 4.71 \\
\hline 3.01 & 3.13 & 3.02 & 3.52 & 4.63 \\
\hline 2.28 & 2.35 & 2.35 & 2.36 & 2.39 \\
\hline $\mathrm{N} / \mathrm{A}$ & $N / A$ & N/A & N/A & $\mathrm{N} / \mathrm{A}$ \\
\hline 3.04 & 3.18 & 2.90 & 2.70 & 5.91 \\
\hline 2.32 & 2.32 & 2.41 & 2.41 & 2.41 \\
\hline 2.64 & 2.58 & 2.98 & 3.86 & 4.31 \\
\hline$N / A$ & 2.35 & 2.80 & 3.50 & 4.31 \\
\hline 2.48 & 2.81 & 2.70 & 3.69 & 4.46 \\
\hline 2.72 & 3.20 & 2.93 & 3.65 & 4.39 \\
\hline 2.34 & 2.82 & 2.48 & 3.16 & 3.90 \\
\hline 2.73 & 2.71 & 2.71 & 3.66 & 4.45 \\
\hline 2.75 & 2.72 & 2.72 & 3.68 & 4.50 \\
\hline 2.79 & 2.79 & 2.68 & 3.61 & 4.48 \\
\hline 3.27 & 3.05 & 3.15 & 4.04 & 6.29 \\
\hline 4.31 & 3.42 & 3.17 & 3.91 & 4.85 \\
\hline$N / A$ & 3.97 & 2.91 & 3.93 & N/A \\
\hline 2.74 & 2.71 & 2.72 & 3.65 & 4.45 \\
\hline 2.68 & 2.67 & 2.68 & 3.51 & 4.17 \\
\hline 2.69 & 2.71 & 2.70 & 3.60 & 4.40 \\
\hline 2.70 & 2.72 & 2.69 & 3.52 & 4.31 \\
\hline 2.83 & 2.44 & 2.71 & 3.64 & 4.60 \\
\hline 2.83 & 2.44 & 2.70 & 3.65 & 4.59 \\
\hline 2.75 & 2.65 & 2.71 & 3.47 & 4.26 \\
\hline 2.73 & 2.93 & 2.65 & 3.46 & 4.14 \\
\hline 2.71 & 2.70 & 2.70 & 3.62 & 4.43 \\
\hline 2.71 & 2.69 & 2.66 & 3.47 & 4.21 \\
\hline 2.80 & 2.71 & 2.70 & 3.63 & 4.43 \\
\hline
\end{tabular}


Page - 7

Attachment A

\section{LONG-TERM IMPORTS}

\section{ACTUALIMPORTS AS A PERCENT OF AUTHORIZED VOLUMES}

IMPORTERIEXPORTER

WISCONSIN PUBLIC SERVICE CORPORATIONTTCGS LId.

YANKEE GAS SERVICES CO./Alberta NE Gas (AEC Oil \& Gas Company)

YANKEE GAS SERVICES CO./Alberta NE Gas (TCGS Lid. 1)

YANKEE GAS SERVICES CO./Aiberta NE Gas (TCGS Ltd. 2)

YANKEE GAS SERVICES CO./Alberta Northeast Gas (ProGas)

YANKEE GAS SERVICES CO./Alberta Northeast Gas (Producers Marketing)

$\begin{array}{ccccc}\begin{array}{c}1999 \\ \text { gtr } \\ \# 3\end{array} & \begin{array}{c}1999 \\ \text { qtr } \\ \# 4\end{array} & \begin{array}{c}2000 \\ \text { gtr } \\ \# 1\end{array} & \begin{array}{c}2000 \\ \text { qtr } \\ \# 2\end{array} & \begin{array}{c}2000 \\ \text { gtr } \\ \# 3\end{array} \\ 88 & 88 & 101 & 98 & 99 \\ 101 & 101 & 101 & 101 & 101 \\ 100 & 100 & \text { N/A } & \text { N/A } & \text { N/A } \\ 100 & 100 & \text { N/A } & \text { N/A } & \text { N/A } \\ 100 & 84 & 100 & 100 & 100 \\ 100 & 100 & 100 & 100 & 100\end{array}$

WEIGHTED AVERAGE PRICE (\$MMBTU) OF IMPORTS

$\begin{array}{ccccc}\begin{array}{c}1999 \\ \text { qtr }\end{array} & \begin{array}{c}1999 \\ \text { qtr }\end{array} & \begin{array}{c}2000 \\ \text { qtr } \\ \# 3\end{array} & \begin{array}{c}2000 \\ \text { qtr } \\ \# 1\end{array} & \begin{array}{c}2000 \\ \text { qtr } \\ \# 3\end{array} \\ & & & & \\ 2.71 & 2.66 & 2.66 & 3.49 & 4.18 \\ 2.80 & 2.98 & 3.15 & 4.15 & 4.65 \\ 2.73 & 2.95 & \text { N/A } & \text { N/A } & \text { N/A } \\ 2.73 & 2.96 & \text { N/A } & \text { N/A } & \text { N/A } \\ 2.85 & 2.99 & 3.25 & 3.93 & 4.71 \\ 2.85 & 3.05 & 3.26 & 3.59 & 4.71\end{array}$




\section{Quarterly Attachment B}

Volumes and Weighted Average Prices

\begin{tabular}{|c|c|c|c|c|c|c|c|c|c|}
\hline \multirow[b]{2}{*}{$\begin{array}{l}\text { Year \& } \\
\text { Month }\end{array}$} & \multicolumn{3}{|c|}{ 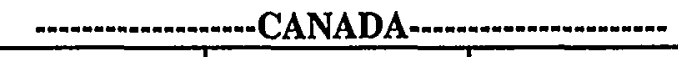 } & \multicolumn{6}{|c|}{ LIQUEFIED NATURAL GAS } \\
\hline & $\begin{array}{l}\text { Number of } \\
\text { Active } \\
\text { Contracts }\end{array}$ & $\begin{array}{c}\text { Volumes } \\
\text { (Mcf) }\end{array}$ & $\begin{array}{l}\text { Weighted } \\
\text { Avg. Price } \\
\text { (\$/MMBtu) }\end{array}$ & $\begin{array}{c}\text { Total } \\
\text { Number of } \\
\text { LNG } \\
\text { Contracts }\end{array}$ & $\begin{array}{l}\text { Algeria } \\
\text { Volumes } \\
\text { (Mcf) }\end{array}$ & $\begin{array}{r}\text { Weight } \\
(\$ / \\
\text { Everett, MA } \\
\text { (Landed Price) }\end{array}$ & $\begin{array}{l}\text { d Avg. Price } \\
\text { IMBtu) } \\
\frac{\text { Lake Charles, LA }}{\text { (Tailgate Price) }}\end{array}$ & $\begin{array}{c}\text { Trinidad } \\
\text { Volumes } \\
\text { (Mcf) }\end{array}$ & $\begin{array}{c}\text { Weighted Avg. Price } \\
\text { (\$/MMBtu) } \\
\text { Everett, MA } \\
\text { (Landed Price) }\end{array}$ \\
\hline \multicolumn{10}{|l|}{1998} \\
\hline January & 211 & $119,517,456$ & 2.29 & 2 & $10,054,192$ & 2.84 & 2.00 & 0 & 0.00 \\
\hline February & 211 & $111,308,950$ & 2.13 & 3 & $7,220,277$ & 2.86 & 2.00 & 0 & 0.00 \\
\hline March & 210 & $120,179,623$ & 2.17 & 1 & $5,166,247$ & 2.84 & 0.00 & 0 & 0.00 \\
\hline April & 208 & $115,076,817$ & 2.23 & 1 & $2,398,524$ & $0.00^{\circ}$ & 2.00 & 0 & 0.00 \\
\hline May & 207 & $111,680,329$ & 2.22 & 2 & $7,533,955$ & 2.44 & 2.00 & 0 & 0.00 \\
\hline June & 205 & $108,359,857$ & 2.07 & 2 & $4,961,946$ & 2.34 & 2.00 & 0 & 0.00 \\
\hline July & 193 & $110,819,028$ & 2.19 & 1 & $3,820,023$ & 0.00 & 2.00 & 0 & 0.00 \\
\hline August & 191 & $108,687,860$ & 1.97 & 1 & $2,205,882$ & 0.00 & 2.00 & 0 & 0.00 \\
\hline September & 192 & $107,592,47.1$ & 1.81 & 1 & $2,445,255$ & 0.00 & 1.57 & 0 & 0.00 \\
\hline October & 191 & $111,552,064$ & 2.02 & 1 & $5,216,505$ & 2.32 & 0.00 & 0 & 0.00 \\
\hline November & 202 & $115,882,342$ & 2.16 & 2 & $4,837,100$ & 2.33 & 2.00 & 0 & 0.00 \\
\hline December & 204 & $112,992,498$ & 2.23 & 2 & $7,500,950$ & 2.37 & 2.00 & 0 & 0.00 \\
\hline Total 1998 & & $1,353,649,295$ & 2.13 & & $63,360,856$ & 2.59 & 1.96 & 0 & 0.00 \\
\hline \multicolumn{10}{|l|}{1999} \\
\hline January & 205 & $122,344,648$ & 2.12 & 2 & $12,790,081$ & 2.29 & 2.00 & 0 & 0.00 \\
\hline February & 205 & $108,873,034$ & 2.02 & 2 & $7,211,930$ & 2.46 & 2.00 & 0 & 0.00 \\
\hline March & 206 & $117,960,699$ & 1.87 & 2 & $12,824,479$ & 2.32 & 2.00 & 0 & 0.00 \\
\hline April & 200 & $108,920,574$ & 1.92 & 2 & $7,180,718$ & 2.25 & 2.00 & 0 & 0.00 \\
\hline May & 197 & $111,168,762$ & 2.25 & 4 & $3,794,081$ & 1.91 & 2.00 & $4,031,987$ & 1.75 \\
\hline June & 196 & $107,532,391$ & 2.20 & 3 & $2,275,512$ & 0.00 & 2.00 & $6,619,872$ & 1.98 \\
\hline
\end{tabular}


Page - 9

\section{Quarterly Attachment B}

Volumes and Weighted Average Prices

CANADA

LIQUEFIED NATURAL GAS -

\begin{tabular}{|c|c|c|c|c|c|c|c|c|c|}
\hline $\begin{array}{l}\text { Year \& } \\
\text { Month }\end{array}$ & 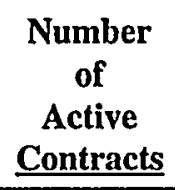 & Volumes (Mcf) & $\begin{array}{c}\text { Weighted } \\
\text { Avg. Price } \\
\text { (\$/MMBtu) } \\
\end{array}$ & $\begin{array}{c}\text { Total } \\
\text { Number of } \\
\qquad \quad \text { LNG } \\
\text { Contracts } \\
\end{array}$ & $\begin{array}{c}\text { Algeria } \\
\text { Volumes } \\
\text { (Mcf) } \\
\end{array}$ & $\begin{array}{r}\text { Weight } \\
(\$ / \\
\text { Everett, MA } \\
\text { (Landed Price) }\end{array}$ & $\begin{array}{l}\text { d Avg. Price } \\
\text { IMBtu) } \\
\frac{\text { Lake Charles, LA }}{\text { (Tailgate Price) }}\end{array}$ & $\begin{array}{c}\text { Trinidad } \\
\text { Volumes } \\
\text { (Mcf) }\end{array}$ & $\begin{array}{c}\text { Weighted Avg. Price } \\
\text { (\$/MMBtu) } \\
\text { Everett, MA } \\
\text { (Landed Price) }\end{array}$ \\
\hline \multicolumn{10}{|l|}{1999} \\
\hline July & 195 & $114,308,602$ & 2.22 & 2 & $2,431,721$ & 0.00 & 2.00 & $6,598,926$ & 2.01 \\
\hline August & 196 & $112,930,969$ & 2.44 & 2 & $2,439,913$ & 0.00 & 2.00 & $5,512,175$ & 2.34 \\
\hline September & 196 & $106,880,296$ & 2.68 & 3 & $4,791,783$ & 2.36 & 2.00 & $2,925,340$ & 2.55 \\
\hline October & 198 & $115,571,379$ & 2.52 & 3 & $4,975,657$ & 2.58 & 2.00 & $1,466,153$ & 2.79 \\
\hline November & 200 & $102,241,097$ & 2.89 & 2 & $2,440,328$ & 2.63 & 0.00 & $5,172,874$ & 2.74 \\
\hline December & 198 & $119,309,295$ & 2.33 & 2 & $2,492,066$ & 2.63 & 0.00 & $5,255,715$ & 2.70 \\
\hline Total 1999 & & $1,348,041,746$ & 2.28 & & $65,648,269$ & 2.36 & 2.00 & $37,583,042$ & 2.30 \\
\hline \multicolumn{10}{|l|}{2000} \\
\hline January & 198 & $121,076,630$ & 2.46 & 3 & $4,721,077$ & 2.63 & 2.00 & $6,295,122$ & 2.88 \\
\hline February & 198 & $112,973,998$ & 2.64 & 2 & $4,987,058$ & 3.34 & 0.00 & $5,167,529$ & 2.77 \\
\hline March & 192 & $113,115,403$ & 2.61 & 3 & $3,796,614$ & 2.59 & 2.00 & $4,804,581$ & 2.73 \\
\hline April & 188 & $108,487,553$ & 2.80 & 2 & $2,182,413$ & 0.00 & 2.42 & $6,636,500$ & 2.93 \\
\hline May & 193 & · $112,313,953$ & 2.93 & 2 & $2,185,402$ & 0.00 & 2.78 & $7,738,983$ & 2.87 \\
\hline June & 193 & $110,274,043$ & 3.71 & 2 & $2,235,627$ & 0.00 & 3.14 & $2,935,927$ & 3.14 \\
\hline July & 195 & $109,321,704$ & 3.92 & 4 & $2,208,289$ & 0.00 & 3.02 & $10,630,936$ & 3.06 \\
\hline August & 192 & $110,077,896$ & 3.52 & 3 & $2,369,684$ & 3.44 & 0.00 & $6,630,295$ & 2.96 \\
\hline September & 192 & $109,165,500$ & $4.06^{\circ}$ & 3 & $2,206,085$ & 0.00 & 3.65 & $4,864,044$ & 2.84 \\
\hline Total 2000 & & $1,005,987,680$ & 3.17 & & $26,892,249$ & 3.11 & 2.71 & $55,703,917$ & 2.92 \\
\hline
\end{tabular}




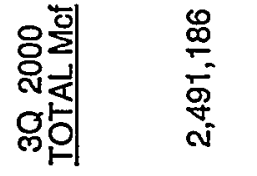

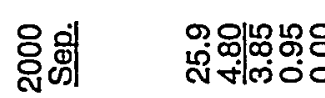

各司 ผ

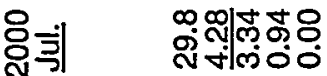

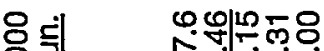

요

तั

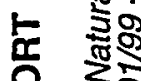

a

앙

우

害

ณ.

늘 ํํㅇ

들 항

웡

要

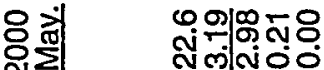

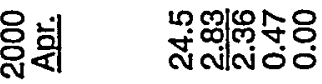

8ू.

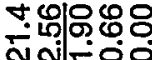

으원

ลงกับ0

+워용요

$<$ 안

눙요

$\frac{1}{5} \sum^{3}$

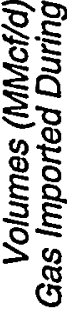

으역

䅛的

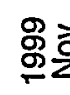

兽。

누엠효

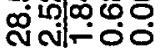

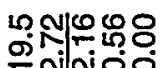

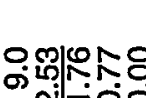

8잉용ㅇㅇ

旁高离

\%ั่

ผึ่

$\bar{\sigma}$

$\frac{8}{8}$

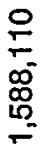

触

영잉융융

ํㅟㅇㅟำ

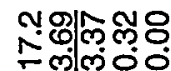

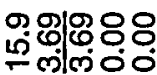

융잉융융

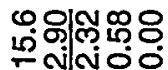

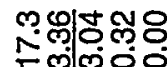

包

잉잉융유

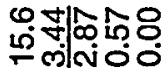

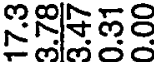

क्रीNo8:

잉잉융유

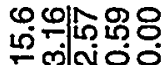

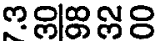

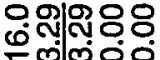

융잉웅웅

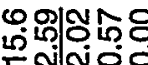

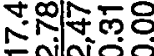

ㅅN승응

윰잉융유

잉이요용

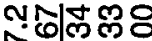

กํำ

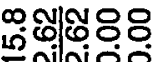

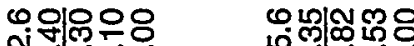

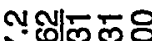

స्ञासंक्ष

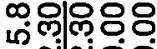

तN-O ogano

또ํํํㅇㅇㅇㅛ

F⿻ำ

위꿍용

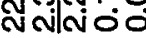

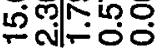

ㄲํำํํ요

సज़่ง०

이요용

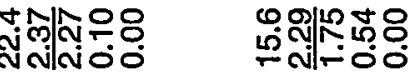

또임응요

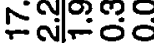

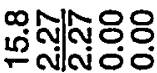

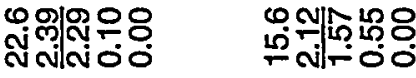

Nㅚㄱํํ용

מNำ

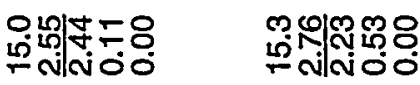

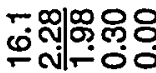

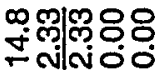

잉잉융유

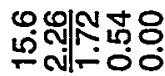

$\stackrel{\infty}{\mathscr{0}}$

$\stackrel{\circ}{\circ}$
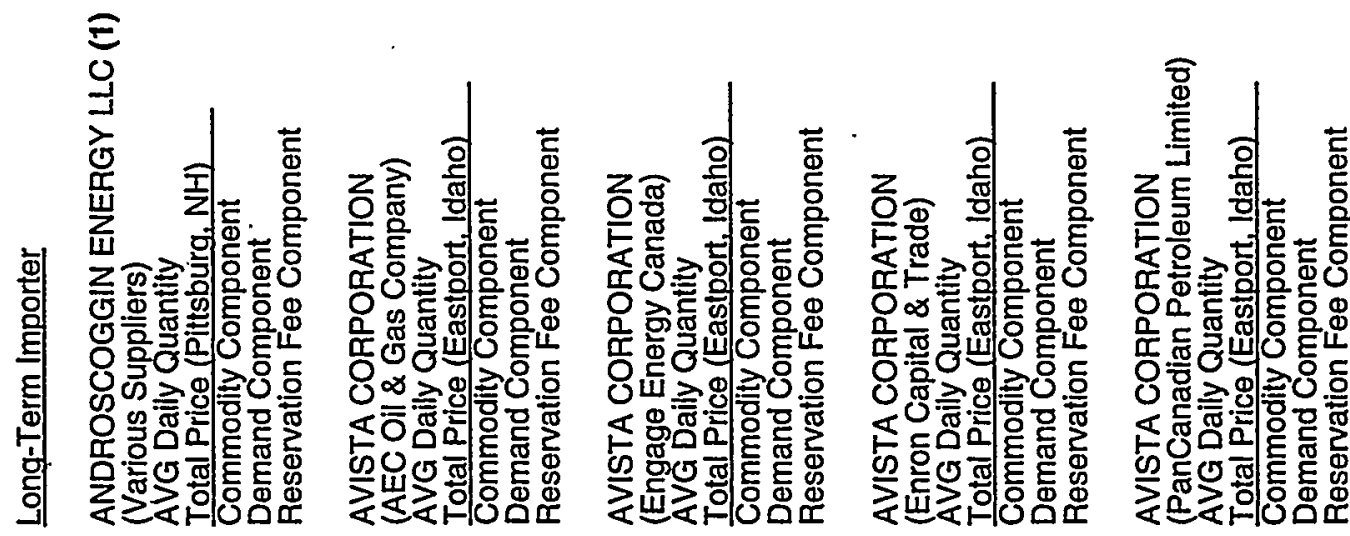


\begin{tabular}{|c|c|c|c|c|c|}
\hline 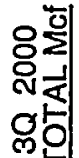 & \begin{tabular}{l}
$\infty$ \\
8 \\
0 \\
\\
\\
\hdashline
\end{tabular} & 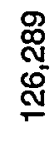 & $\begin{array}{l}\mathbb{N} \\
\mathbb{0} \\
\mathbb{N} \\
\mathbb{N}\end{array}$ & 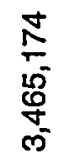 & 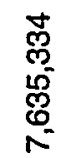 \\
\hline
\end{tabular}

\begin{tabular}{|c|c|c|c|c|}
\hline 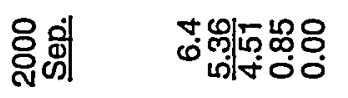 & 임잉ㅇㅇㅇㅇㅇ & 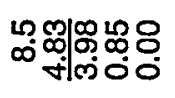 & 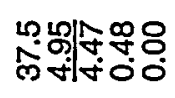 & 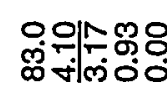 \\
\hline 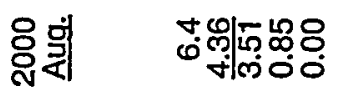 & 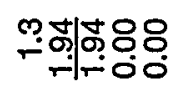 & 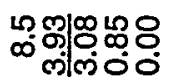 & 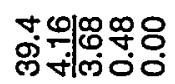 & 웅 \\
\hline
\end{tabular}

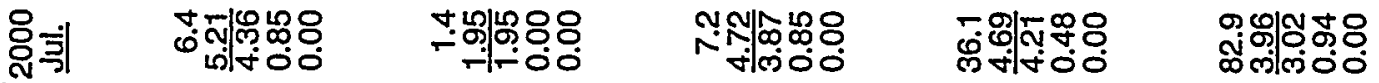

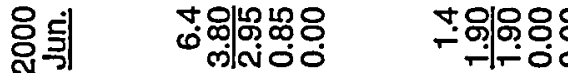

นกำ

시시요용

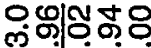

மீ이용

무잉ㅇㅇㅇ

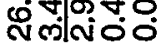

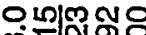

गิ

을

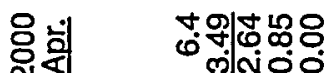

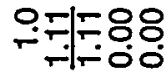

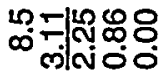

베용요

nต

똔ำ

$=\quad$ แ

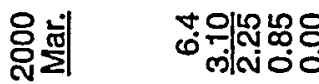

부의응ㅇㅇㅇㅇㅇㅇ

ம

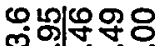

둥형ㅇㅇ

들 통

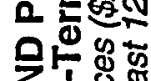

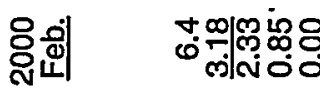

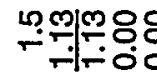

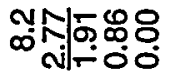

두임용ㅇㅇ

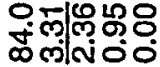

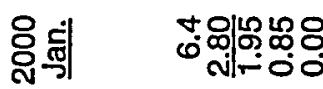

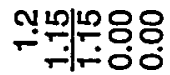

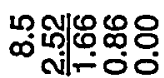

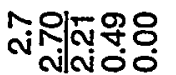

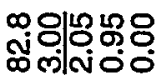

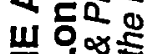

용

부ㅇㅟㅠ용

띠용ㅇㅇ

象

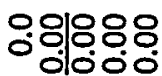

이유용요

용ํํ

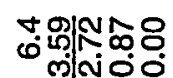

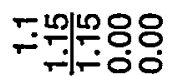

ㄴํํํํ요

웅잉용ㅇㅇㅇㅇㅇ

이요용요

畐히

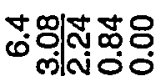

아다퉁ㅇㅇ

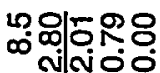

:잉영:

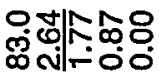

章해 :

๓

$\infty$

ఖ্ণ

ஜு
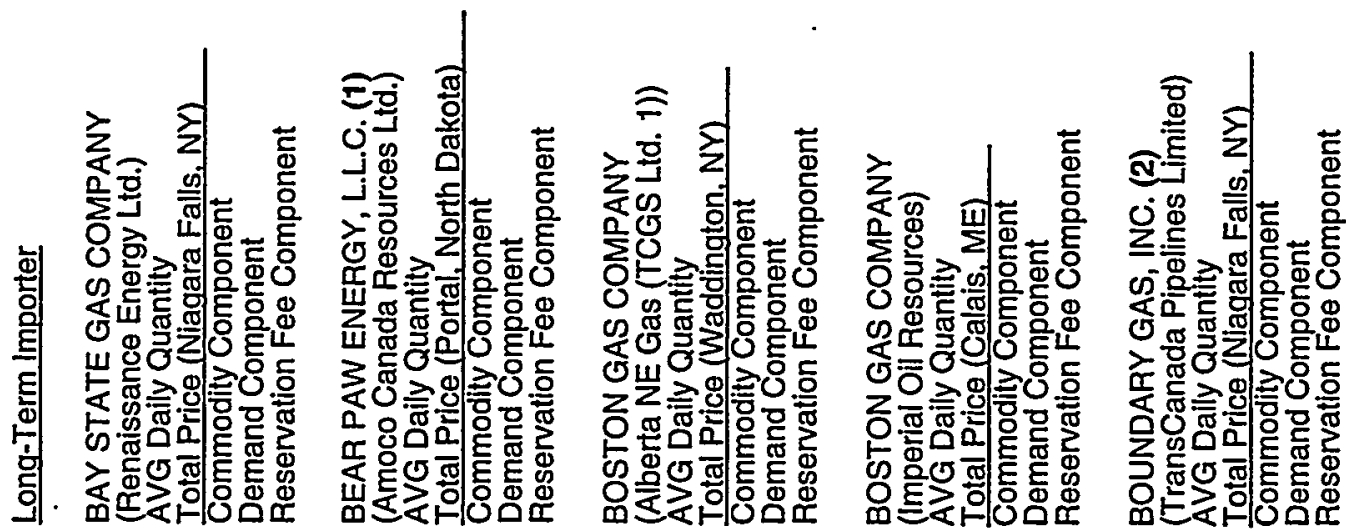


\section{VOLUME AND PRICE REPORT}

\section{Long-Term Imports}

Volumes (MMct/d) \& Prices (\$/MMBTU) of Natural

Gas Imported During the Past 12 Months 10/01/99 - 09/30/00

$\begin{array}{ll}\text { Long-Term Importer } & \text { Auth } \\ \text { BROOKLYN NAVY YARD COGEN. PARTNERS, L.P }\end{array}$

(Crestar Energy)
AVG Daily Quantity

Total Price (Waddington, NY)

Commodity Component

Demand Component

Reservation Fee Component

\begin{tabular}{ll}
10.9 & 10.9 \\
3.08 & 3.53 \\
\hline 2.15 & 2.57 \\
0.93 & 0.96 \\
0.00 & 0.00
\end{tabular}

$\begin{array}{ll}1999 & 2000 \\ \text { Dec. } & \text { Jan. }\end{array}$

20002000

2000
Apr.

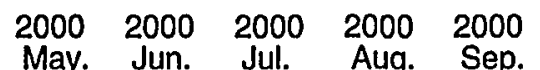

302000

TOTAL MCF

YTD

Auth.
Vols.

BROOKLYN NAVY YARD COGEN. PARTNERS, L.P.

(PanCanadlan Petroleum Ltd.)

AVG Daily Quantity

15.0

\begin{tabular}{lll}
16.4 & 16.3 & 16.2 \\
3.03 & 3.48 & 2.60 \\
\hline 2.10 & 2.52 & 1.67 \\
0.93 & 0.96 & 0.93
\end{tabular}

\begin{tabular}{ll}
10.8 & 10.8 \\
2.65 & 2.88 \\
\hline 1.72 & 1.88 \\
0.93 & 1.00 \\
0.00 & 0.00
\end{tabular}

10.8
2.88
1.88
1.00
0.00

10.8
$\frac{3.18}{2.12}$
1.06
0.00

10.9
$\frac{3.09}{2.09}$
1.00
0.00

$\begin{array}{ll}10.9 & 10.8 \\ 3.48 & 3.63\end{array}$

$\frac{3.48}{2.45} \cdot \frac{3.63}{2.63}$

$\begin{array}{ll}1.03 & 1.00 \\ 0.00 & 0.00\end{array}$

10.7
4.74

10.8
5.01

10.9 .10 .8

$\begin{array}{llll}\frac{4.74}{3.71} & \frac{5.01}{4.01} & \frac{4.24}{3.24} & \frac{5.14}{4.11} \\ 1.03 & 1.00 & 1.00 & 1.03\end{array}$

$\begin{array}{llll}1.03 & 1.00 & 1.00 & 1.03 \\ 0.00 & 0.00 & 0.00 & 0.00\end{array}$

997,261

$2,968,629$

Commodity Component

Demand Component

Reservation Fee Component

$0.00 \quad 0.00$
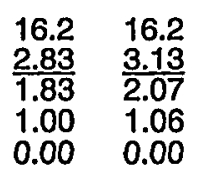

16.4
$\frac{3.04}{2.04}$
1.00

16.4
$\frac{3.43}{2.40}$
1.03
0.00

16.2
3.58

BROOKLYN UNION GAS COMPANY

(Alberta NE Gas (AEC Oil \& Gas Company))

AVG Daily Quantity

4.0

\begin{tabular}{rr}
4.0 & 4.0 \\
2.94 & 3.34 \\
\hline 2.06 & 2.46 \\
0.88 & 0.88 \\
0.00 & 0.00
\end{tabular}

4.0
2.67
1.79
0.88

4.0
2.97
2.03

2.97
0.03

$\frac{3.32}{2.38}$

$\begin{array}{lll}0.88 & 0.94 & 0.94 \\ 0.00 & 0.00 & 0.00\end{array}$

\begin{tabular}{rr}
4.0 & 0.0 \\
4.31 & 0.00 \\
\hline 3.37 & 0.00 \\
0.94 & 0.00 \\
0.00 & 0.00
\end{tabular}

$\begin{array}{r}4.0 \\ 3.57 \\ \hline 2.65\end{array}$

2.65

Commodity Component

Reservation Fee Component

\section{BROOKLYN UNION GAS COMPANY}

(Alberta NE Gas (TCGS Lid. 1))

AVG Daily Quantity

Total Price (Waddington, NY)

Commodity Component

Demand Component

Reservation Fee Component

$\begin{array}{llllllllllll}24.0 & 24.0 & 24.0 & 24.0 & 24.0 & 24.0 & 24.0 & 24.0 & 24.0 & 20.1 & 24.0 & 24.0 \\ \frac{2.80}{2.01} & \frac{3.22}{2.43} & \frac{2.36}{1.57} & \frac{2.52}{1.66} & \frac{2.77}{1.91} & \frac{2.75}{1.89} & \frac{3.11}{2.25} & \frac{3.30}{2.46} & \frac{4.42}{3.57} & \frac{4.72}{3.87} & \frac{3.93}{3.08} & \frac{4.83}{3.98} \\ 0.79 & 0.79 & 0.79 & 0.86 & 0.86 & 0.86 & 0.86 & 0.84 & 0.85 & 0.85 & 0.85 & 0.85 \\ 0.00 & 0.00 & 0.00 & 0.00 & 0.00 & 0.00 & 0.00 & 0.00 & 0.00 & 0.00 & 0.00 & 0.00\end{array}$

\section{BROOKLYN UNION GAS COMPANY}

(Alberta NE Gas (TCGS Lid. 2))

AVG Daily Quantity

Total Price (Waddington, NY)

Commodity Componen

Demand Component

Reservation Fee Component

$\begin{array}{lllllllllllll}18.0 & 18.0 & 18.0 & 18.0 & 18.0 & 18.0 & 18.0 & 18.0 & 18.0 & 15.1 & 18.0 & 18.0 \\ \frac{2.80}{2.01} & \frac{3.22}{2.43} & \frac{2.36}{1.57} & \frac{2.52}{1.66} & \frac{2.77}{1.91} & \frac{2.75}{1.89} & \frac{3.11}{2.25} & \frac{3.30}{2.46} & \frac{4.42}{3.57} & \frac{4.72}{3.87} & \frac{3.93}{3.08} & \frac{4.83}{3.98} \\ 0.79 & 0.79 & 0.79 & 0.86 & 0.86 & 0.86 & 0.86 & 0.84 & 0.85 & 0.85 & 0.85 & 0.85 \\ 0.00 & 0.00 & 0.00 & 0.00 & 0.00 & 0.00 & 0.00 & 0.00 & 0.00 & 0.00 & 0.00 & 0.00\end{array}$

$1,566,000$

$4,842,000$ 


\section{VOLUME AND PRICE REPORT}

\section{Long-Term Imports}

Volumes (MMcf/d) \& Prices (\$MMBTU) of Natural

Gas Imported During the Past 12 Months 10/01/99 - 09/30/00

Long-Term Importer

BROOKLYN UNION GAS COMPANY

(Alberta Northeast Gas (ProGas))

AVG Dally Quantity

Total Price (Waddington, NY)

Commodity Component

Demand Component

Reservation Fee Component

BROOKLYN UNION GAS COMPANY

(Alberta Northeast Gas (Producers Marketing))

AVG Daily Quantity

Total Price (Waddington, NY)

Commodity Component

Demand Component

Reservation Fee Component

BROOKLYN UNION OF LONG ISLAND (1)

(Alberta NE Gas (AEC Oll \& Gas))

AVG Daily Quantity

Total Price (Waddington, NY)

Commodily Component

Demand Component

Reservation Fee Component

BROOKLYN UNION OF LONG ISLAND (2)

(Alberta NE Gas (TCGS Ltd. 1))

AVG Daily Quantity

Total Price (Waddington, NY)

Commodity Component

Demand Componen

Reservation Fee Component

BROOKLYN UNION OF LONG ISLAND (3)

(Alberta NE Gas (TCGS Ltd. 2))

AVG Daily Quantity

Total Price (Waddington, NY)

Commodity Component

Demand Component

Reservation Fee Component

(1) Successor to Long Island Lighting Company.

(3) Successor to Long Island Lighting Company.
Oct. Nov. Dec.

Jan.

2000

20002000

$\begin{array}{lllll}2000 & 2000 \quad 2000 \quad 2000 \quad 2000\end{array}$

15.8

\begin{tabular}{lrll}
15.8 & 8.2 & 15.8 & 15.8 \\
2.99 & 3.38 & 2.79 & 2.98 \\
\hline 2.07 & $\frac{2.48}{1.87}$ & 1.96 \\
0.92 & 0.90 & 0.92 & 1.02 \\
0.00 & 0.00 & 0.00 & 0.00
\end{tabular}

15.8
$\frac{3.42}{2.40}$
1.02

\begin{tabular}{l}
15.8 \\
3.36 \\
\hline 2.35 \\
1.01
\end{tabular}

15.8
$\frac{3.49}{2.47}$
1.02

$\begin{array}{ll}15.8 & 15.8 \\ 3.61 & 4.70\end{array}$

$\frac{3.61}{2.61} \quad \frac{4.70}{3.69}$

$\begin{array}{ll}1.00 & 1.01\end{array}$

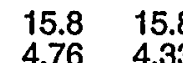

\begin{tabular}{ll}
15.8 & 15.8 \\
4.33 & 5.05 \\
\hline & 4.05
\end{tabular}

$1.00 \quad 1.00$

0.00

8.2

$\begin{array}{rrrrr}8.2 & 8.2 & 8.2 & 8.2 & 8.2 \\ \frac{2.99}{2.10} & \frac{3.37}{2.44} & \frac{2.79}{1.90} & \frac{2.98}{1.99} & \frac{3.42}{2.44} \\ 0.89 & 0.93 & 0.89 & 0.99 & 0.98\end{array}$

$\begin{array}{lllll}0.89 & 0.93 & 0.89 & 0.99 & 0.98 \\ 0.00 & 0.00 & 0.00 & 0.00 & 0.00\end{array}$

8.2
$\frac{3.37}{2.39}$
0.98

$\frac{3.29}{2.51}$

$\begin{array}{ll}0.08 & 0.98 \\ 0.00\end{array}$

8.2
$\frac{3.60}{2.64}$
0.96

$\begin{array}{r}8.2 \\ 3.69 \\ \hline 2.72\end{array}$

$\frac{3.69}{2.72}$

0.00

0.00

3.1

$\begin{array}{rr}3.1 & 1.4 \\ \frac{2.94}{2.06} & \frac{3.34}{2.46} \\ 0.88 & 0.88 \\ 0.00 & 0.00\end{array}$

3.1
2.67
1.79

3.1
$\frac{2.97}{2.03}$
0.94

\begin{tabular}{r}
3.1 \\
3.32 \\
\hline 2.38 \\
0.94 \\
0.00
\end{tabular}

0.0
0.00
0.00
0.00
0.00

$\begin{array}{r}0.0 \\ 0.00 \\ \hline .00\end{array}$

$0.00 \quad 0.00$

0.00

0.00

$0.00 \quad 0.00$
0

3.1
$\frac{3.57}{2.65}$
0.92
0.00

3.1
4.72
3.79

0.93
0.00

$\begin{array}{r}3.1 \\ 4.71 \\ \hline 3.78\end{array}$

3.78

0.93
0.00

$\begin{array}{r}3.1 \\ 4.33 \\ \hline 3.41\end{array}$

30.4

\begin{tabular}{ll}
30.4 & 19.3 \\
2.80 & $\frac{3.22}{2.43}$ \\
\hline 2.01 & 1 \\
0.79 & 0.79 \\
0.00 & 0.00
\end{tabular}

$30.4 \quad 30.4$

$30.4 \quad 30.4$

$\frac{2.36}{1.57} \quad \frac{2.52}{1.66}$

$\frac{2.77}{1.91}$

30.4
$\frac{2.75}{1.89}$

30.4

30.4

30.4

$\begin{array}{ll}0.79 & 0.86 \\ 0.00 & 0.00\end{array}$

$\begin{array}{ll}0.00 & 0.86 \\ 0.00\end{array}$

$\frac{3.11}{2.25}$

2.46

0.84
0.00

$\frac{4.42}{3.57}$

3.57
0.85
0.00

25.5
4.72

$\frac{4.72}{3.87}$

30.4

$\begin{array}{lll}0.85 & 0.85 & 0.85\end{array}$

13.5

\begin{tabular}{lr}
13.5 & 8.6 \\
2.80 & $\frac{3.22}{2.43}$ \\
\hline 2.01 & 1 \\
0.79 & 0.79 \\
0.00 & 0.00
\end{tabular}

13.5
2.36
1.57
0.79
0.00

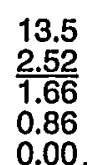

13.5
2.77
1.91
0.86
0.00

13.5
2.75
1.89
0.86
0.00

13.5
3.11
2.25
0.86
0.00

\begin{tabular}{l}
13.5 \\
3.30 \\
\hline 2.46 \\
0.84 \\
0.00
\end{tabular}

13.5
4.42
3.57
0.85
0.00

11.3
4.72
3.87
0.85
0.00

\begin{tabular}{l}
13.5 \\
3.93 \\
\hline
\end{tabular}
$3 Q 2000$

TOTAL MC

$1,457,280$

$\frac{5.05}{4.05}$

0.00

30.4

4.83

3.98

0.00

$2,644,800$

$8,177,600$

754,95

$2,248,444$

281,980

652,845

$3,631,500$

YTD

OTAL Mcf

$4,340,160$

13.5

$\frac{4.83}{3.98}$

0.85

0.00 


\section{VOLUME AND PRICE REPORT}

Long-Term Imports

Volumes (MMcf/d) \& Prices (\$MMBTU) of Natural

Gas Imported During the Past 12 Months 10/01/99 - 09/30/00

\section{Long-Term Importer}

BAROOKLYN UNION OF LONG ISLAND (1)

(Alberta Northeast Gas (ProGas))

AVG Daily Quantity

Total Price (Waddington, NY)

Commodity Component

Demand Component

Reservation Fee Component

BROOKLYN UNION OF LONG ISLAND (2)

(Alberta Northeast Gas (Producers Marketing))

AVG Daily Quantity

Total Price (Waddington, NY)

Reservation Fee Component

CASCADE NATURAL GAS COPORATION (3)

(IGI Resources, Inc.)

AVG Daily Quantity

Total Price (Eastport, Idaho)

Commodity Componen

Demand Component

Reservation Fee Component

CASCADE NATURAL GAS CORPORATION

(Duke Energy Trad. \& Mktg. Svcs., LLC)

AVG Daily Quantity

Total Price (Sumas, Washington)

\section{Commodity Componen}

Demand Component

Reservation Fee Component

\section{CASCADE NATURAL GAS CORPORATION}

(Engage Energy Canada)

AVG Daily Quantity

Total Price (Eastport, Idaho)

Commodity Componen

Demand Component
Commodity Componen

Demand Component

Reservation Fee Component

\section{Auth}

Vols. $\quad 19991999$

Oct. Nov 19992000

11.9

\begin{tabular}{lrrr}
11.9 & 3.7 & 11.9 & 11.9 \\
2.99 & 3.38 & 2.79 & 2.98 \\
\hline 2.07 & $\frac{2.48}{2.48}$ & $\frac{1.87}{1.96}$ \\
0.92 & 0.90 & 0.92 & 1.02 \\
0.00 & 0.00 & 0.00 & 0.00
\end{tabular}

\begin{tabular}{l}
11.9 \\
3.42 \\
\hline 2.40 \\
1.02
\end{tabular}

6.2

\begin{tabular}{rr}
6.2 & 3.7 \\
2.99 & 3.37 \\
\hline 2.10 & $\frac{3.44}{2.40}$ \\
0.89 & 0.93
\end{tabular}

6.2
2.79
1.90

6.2
2.98
1.99

1.99

$\begin{array}{llll}0.00 & 0.00 & 0.00 & 0.00\end{array}$

\begin{tabular}{r}
6.2 \\
3.42 \\
\hline 2.44 \\
0.98
\end{tabular}

0.00

6.2
$\frac{3.37}{2.39}$
0.98

0.98
0.00

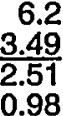

$\frac{3.49}{2.51}$

0.98
0.00

$\begin{array}{r}6.2 \\ 3.60 \\ \hline\end{array}$

$\frac{3.60}{2.64}$

0.96

0.00

$\begin{array}{r}6.2 \\ 3.69 \\ \hline\end{array}$

2.72

0.97

0.00

7.4

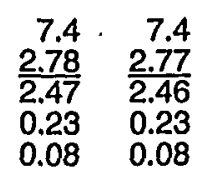

\begin{tabular}{lr}
7.4 & 7.4 \\
2.77 & 2.79 \\
\hline 2.46 & 2.45 \\
0.23 & 0.26 \\
0.08 & 0.08
\end{tabular}

\begin{tabular}{r}
6.4 \\
2.90 \\
\hline 2.49 \\
0.32
\end{tabular}

6.5
2.87

$\frac{2.87}{2.49}$

0.0
0.00
0.00

0.0
0.00

$\frac{0.00}{0.00}$

0.0
0.00

$\frac{0.00}{0.00}$

0.09

$0.00 \quad 0.00$

0.00

$\frac{0.00}{0.00}$

0.00
0.00

$\begin{array}{r}6.2 \\ 4.75 \\ \hline 3.78\end{array}$

3.78
0.97

0.97
0.00

$\frac{4.33}{3.36}$

3.36
0.97

0.00

5.06

4.09

0.97
0.00

12.0

\begin{tabular}{lr}
11.8 & 8.5 \\
2.56 & 3.13 \\
\hline 1.87 & $\frac{2.38}{2.38}$ \\
0.52 & 0.54
\end{tabular}

11.8
2.44
1.75
0.53

\begin{tabular}{l}
11.8 \\
2.46 \\
\hline 1.76
\end{tabular}

8.8
2.52
1.75

\begin{tabular}{l}
11.8 \\
2.47 \\
\hline 1.76 \\
0.55
\end{tabular}

$\begin{array}{ll}0.17 & 0.21\end{array}$

0.54
0.16

0.61
0.16

0.55
0.16

\begin{tabular}{l}
11.8 \\
2.98 \\
\hline 2.15 \\
0.58 \\
0.25
\end{tabular}

11.8
.299

$\frac{2.99}{2.20}$

\begin{tabular}{l}
11.7 \\
3.97 \\
\hline 3.07
\end{tabular}

$\frac{3.97}{3.07}$

\begin{tabular}{l}
11.6 \\
5.38 \\
\hline 4.44
\end{tabular}

0.54
0.25

0.57
0.33

0.0

$\underline{0.00}$

0.0

$\frac{0.00}{0.00}$

$0.00 \quad 0.00$

$0.00 \quad 0.00$
0

33.2

\begin{tabular}{lll}
26.3 & 25.9 & 25.6 \\
2.13 & 2.75 & 2.70 \\
\hline 1.76 & $\frac{2.36}{2.32}$ \\
0.26 & 0.28 & 0.27 \\
0.11 & 0.11 & 0.11
\end{tabular}

26.5
2.74
2.33
0.30
0.11

\begin{tabular}{l}
26.5 \\
2.76 \\
\hline 2.33 \\
0.32 \\
0.11
\end{tabular}

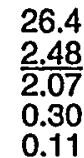

26.3

$\frac{2.50}{2.09}$

0.30
0.11
26.4

$\frac{2.80}{2.38}$

0.31

0.11
26.2

$\frac{3.32}{2.91}$

0.30
0.11 $\frac{0.00}{0.00}$

$3 Q 2000$

TOTAL MCf

$1,092,960$

$3,255,120$

566,260

$1,686,470$

(1) Successor to Long Island Llighting Company

(2) Successor to Long Island Lighting Company.

(3) This contract is for winter-only supplies. 


\section{VOLUME AND PRICE REPORT}

\section{Long-Term Imports}

Volumes (MMct/d) \& Prices (\$MMBTU) of Natural

Gas Imported During the Past 12 Months 10/01/99 - 09/30/00
Long-Term Importer
CASCADE NATURAL GAS CORPORATION (1)
(Engage Energy Canada)

AVG Daily Quantity

Total Price (Sumas, Washington)

Commodity Component

Reservation Fee Component

\section{CENTRAL HUDSON COMPANY}

(Alberta NE Gas (AEC Oil \& Gas Company))

AVG Daily Quantity

Total Price (Waddington, NY)

Demand Component

Reservation Fee Component

CENTRAL HUDSON COMPANY

(Alberta NE Gas (TCGS Ltd. 1))

AVG Daily Quantity

Total Price (Waddington, NY)

Commodity Component

Demand Component

Reservation Fee Component

CENTRAL HUDSON COMPANY

(Alberta NE Gas (TCGS Ltd. 2))

AVG Daily Quantity

Total Price (Waddington, NY).

Commodity Component

Demand Component

Reservation Fee Component

CENTRAL HUDSON COMPANY

(Alberta Northeast Gas (ProGas))

AVG Daily Quantity

Total Price (Waddington, NY)

Commodity Componen

Demand Component

Reservation Fee Component

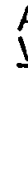

$\begin{array}{llll}\text { Auth. } & 1999 & 1999 & 1999 \\ \text { Vols. } & \text { Oct. } & \text { Nov. } & \text { Dec. }\end{array}$

Dec.

2000

2000

2000

2000

\begin{tabular}{ll}
2000 & 2000 \\
Mav. & Jun. \\
\hline
\end{tabular}

$20002000 \quad 2000$

Aug. Sep.

$3 Q 2000$

TOTAL MC

30.0

$\begin{array}{ll}14.7 & 19 . \\ 2.39 & 2.39 \\ 0.00 & 0.00 \\ 0.00 & 0.00\end{array}$

$19.6 \quad 29.4$

$\frac{2.97}{2.97} \quad \frac{2.71}{2.71}$

0.00

0.00

\begin{tabular}{l}
19.6 \\
2.35 \\
\hline 2.35 \\
0.00 \\
0.00
\end{tabular}

$29.4 \quad 16.2$

$\begin{array}{ll}29.4 & 16.2 \\ \frac{2.41}{2.41} & \frac{2.36}{2.36}\end{array}$

2.41

0.00

6.7
2.73
2.73
0.00
0.00

0.0
0.00

$\frac{0.00}{0.00}$

0.00
0.00

0.0
0.00
0.00

0.00
0.00
0.00

0.00

0.00
0.00
0.00

0.0

$\frac{0.00}{0.00}$

0.00
0.00

$\begin{array}{ll}0.00 & 0.00 \\ 0.00 & 0.00 \\ 0.00 & 0.00\end{array}$

0.1

$\begin{array}{rrrrr}0.1 & 0.0 & 0.1 & 0.1 & 0.1 \\ \frac{2.94}{2.06} & \frac{3.34}{2.46} & \frac{2.67}{1.79} & \frac{2.97}{2.03} & \frac{3.32}{2.38} \\ 0.88 & 0.88 & 0.88 & 0.94 & 0.94 \\ 0.00 & 0.00 & 0.00 & 0.00 & 0.00\end{array}$

0.1
4.31
3.37
0.94
0.00

0.1
$\frac{3.44}{2.51}$
0.93

0.1
3.57
2.65
0.92
0.00

0.2
4.72
3.79

4.72
3.79
0.93

0.1
4.71
3.78

4.71
3.78
0.93
0.00

$\begin{array}{r}0.1 \\ 4.33 \\ \hline 3.41\end{array}$

$\frac{4.33}{3.41}$

0.92
0.00

0.1
4.92
4.00

0.92

8,832

0

$2,167,008$

14.1

\begin{tabular}{rr}
14.1 & 8.9 \\
2.80 & 3.22 \\
\hline 2.01 & 2.43 \\
0.79 & 0.79 \\
0.00 & 0.00
\end{tabular}

14.1
2.36
1.57
0.79
0.00

\begin{tabular}{l}
14.1 \\
2.52 \\
\hline 1.66 \\
0.86
\end{tabular}

14.1
$\frac{2.77}{1.91}$
0.86

14.1
$\frac{2.75}{1.89}$
0.86

14.1
$\frac{3.11}{2.25}$
0.86
0.00

14.1
$\frac{3.30}{2.46}$
0.84

$\begin{array}{lll}14.1 & 11.8 & 14.1 \\ 4.42 & 4.72 & 3.93\end{array}$

$\begin{array}{lll}\frac{4.42}{3.57} & \frac{4.72}{3.87} & \frac{3.93}{3.08}\end{array}$

$\begin{array}{lll}0.85 & 0.85 & 0.08\end{array}$

$\begin{array}{lll}0.00 & 0.00 & 0.00\end{array}$

14.1

$\frac{4.83}{3.98}$

0.85

$1,226,700$

$3,792,900$

1.5

$\begin{array}{rr}1.5 & 0.9 \\ \frac{2.80}{2.01} & \frac{3.22}{2.43} \\ 0.79 & 0.79\end{array}$

1.5
2.36
1.57
0.79

$\begin{array}{lr}\frac{1.5}{1.52} & \frac{1.5}{1.77} \\ 0.86 & 0.91 \\ 0.00 & 0.86\end{array}$

$\frac{2.75}{1.89}$

0.00

0.00

0.00

1.3

$\begin{array}{rrrr}1.3 & 2.1 & 1.3 & 1.3 \\ \frac{2.99}{2.07} & \frac{3.38}{2.48} & \frac{2.79}{1.87} & \frac{2.98}{1.96} \\ 0.92 & 0.90 & 0.92 & 1.02 \\ 0.00 & 0.00 & 0.00 & 0.00\end{array}$

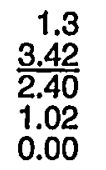

1.3
$\frac{3.36}{2.35}$
1.01
0.00

\begin{tabular}{r}
1.3 \\
3.49 \\
\hline 2.47 \\
1.02 \\
0.00
\end{tabular}

1.3
3.61
2.61
1.00
0.00

1.3
4.70
3.69
1.01
0.00

$\begin{array}{r}1.3 \\ 4.76 \\ \hline .75\end{array}$

4.76
3.75
1.01

1.01
0.00

4.33

3.33
1.00

0.00

$\frac{5.05}{4.05}$

1.00

0.00

(1) Sumas-Cascade Pipeline: Ocl(147,200),Nov(268,825),Dec(247,792),Jan(282,684),Feb(257,433),Mar(198,615),Apr(201,961). Beginning on 11/1/99, the daily contract demand increased to 30 MMcf. 


\section{VOLUME AND PRICE REPORT}

\section{Long-Term Imports}

Volumes (MMcf/d) \& Prices (\$/MMBTU) of Natural

Gas Imported During the Past 12 Months 10/01/99 - 09/30/00

Long-Term Importer

Auth. $\quad 1999 \quad 1999 \quad 1999$

$\begin{array}{lllllll}\text { Auth. } & 1999 & 1999 & 1999 & 2000 & 2000 & 2000 \\ \text { Vols. } & \text { Oct. } & \text { Nov. } & \text { Dec. } & \text { Jan. } & \text { Feb. } & \text { Mar. }\end{array}$

Feb.

Mar. Apr.

2000

3.0

\begin{tabular}{rrr}
3.0 & 2.1 & 3.0 \\
2.99 & $\frac{3.37}{2.44}$ & $\frac{2.79}{1.90}$ \\
\hline 2.10 & 2.44 \\
0.89 & 0.93 & 0.89 \\
0.00 & 0.00 & 0.00
\end{tabular}

3.0
2.98

$\frac{2.98}{1.99}$

3.0

$\frac{3.42}{2.44}$

0.00

0.00

3.0
3.37

$\frac{3.37}{2.39}$

Demand Component

Reservation Fee Component

CHEVRON NATURAL GAS SERVICES, INC. (1)

(Chevron Canada Resources)

AVG Daily Quantity

22.0

$\begin{array}{llll}18.7 & 18.3 & 16.7 & 19.4 \\ \frac{2.45}{2.11} & \frac{2.59}{2.23} & \frac{2.61}{2.23} & \frac{2.58}{2.23} \\ 0.34 & 0.36 & 0.38 & 0.35 \\ 0.00 & 0.00 & 0.00 & 0.00\end{array}$

$0.00 \quad 0.00$

Demand Component

Reservation Fee Component

CHEVRON U.S.A. INC. (2)

(Chevron Canada Resources)

AVG Daily Quantity

Total Price (Eastport, Idaho)

Demand Componen

Reservation Fee Component

CHEVRON U.S.A. INC. (3)

(Chevron Canada Resources)

AVG Daily Quantity

Total Price (Champlain, NY)

Demand Component

Reservation Fee Component

CITY OF BURBANK, CALIFORNIA (4)

(Engage Energy Canada, L.P.)

AVG Daily Quantity

Total Price (Eastport, Idaho)

Commodity Componen

Demand Component

Reservation Fee Component

$\begin{array}{llll}60.3 & 61.0 & 61.4 & 61.6 \\ 2.15 & 2.61 & 2.03 & \frac{2.06}{2.06} \\ \frac{2.15}{2.61} & \frac{2.03}{2.06} \\ 0.00 & 0.00 & 0.00 & 0.00\end{array}$

$0.00 \quad 0.00$

0.00

0.00

1.5

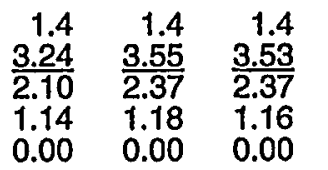

\begin{tabular}{r}
1.5 \\
3.58 \\
\hline 2.37 \\
1.21 \\
0.00
\end{tabular}

1.6
3.61
2.37
1.24
0.00

\begin{tabular}{r}
1.8 \\
3.42 \\
\hline 2.37 \\
1.05 \\
0.00
\end{tabular}

$\begin{array}{r}1.6 \\ 3.63 \\ \hline 2.37\end{array}$

2.37
1.26

3.0
3.49

$\frac{3.49}{2.51}$

3.0
$\frac{3.60}{2.64}$
0.96

0.00

0.00

0.00

1.8.7 14.3

15.9

0.48

0.43

$\frac{2.64}{2.23}$

0.41

\begin{tabular}{ll}
61.4 & 61.0 \\
2.18 & 2.19 \\
\hline 2.18 & 2.19 \\
0.00 & 0.00
\end{tabular}

\begin{tabular}{l}
58.9 \\
2.56 \\
\hline 2.56
\end{tabular}

\begin{tabular}{l}
61.0 \\
2.60 \\
\hline 2.60
\end{tabular}

$0.00 \quad 0.00$

4.8

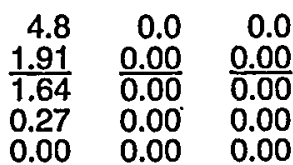

\begin{tabular}{r}
0.0 \\
0.00 \\
\hline 0.00 \\
0.00 \\
0.00
\end{tabular}

\begin{tabular}{r}
0.0 \\
0.00 \\
\hline 0.00 \\
0.00 \\
0.00
\end{tabular}

\begin{tabular}{r}
0.0 \\
0.00 \\
\hline 0.00 \\
0.00 \\
0.00
\end{tabular}

\begin{tabular}{r}
0.0 \\
0.00 \\
\hline 0.00 \\
0.00 \\
0.00
\end{tabular}

$\begin{array}{r}0.0 \\ 0.00 \\ \hline\end{array}$

$\frac{0.00}{0.00}$
Commodity Componen

3.69

0.00

$\begin{array}{r}3.0 \\ 4.75 \\ \hline\end{array}$

3.78

0.00

$\frac{4.33}{3.36}$

0.97

5.06

\begin{tabular}{l}
.5 .06 \\
\hline 4.09 \\
0.97
\end{tabular}

0.00

18.9

18.2

2.23
0.37

$\frac{2.61}{2.23}$

2.23
0.38

\begin{tabular}{ll}
18.1 & 18.5 \\
2.61 & 2.62 \\
\hline 2.23 & 2.23 \\
0.38 & 0.39 \\
0.00 & 0.00
\end{tabular}

$1,680,902$

$4,836,118$

274,528

817,616

\begin{tabular}{l}
61.0 \\
3.49 \\
\hline 3.49
\end{tabular}

$\begin{array}{r}61.5 \\ 3.82 \\ \hline 3.82\end{array}$

$\begin{array}{r}61.6 \\ 3.30 \\ \hline\end{array}$

$\frac{3.30}{3.30}$

61.2

4.50

0.00

0.00
0.00

$0.00 \quad 0.00$

0.00

$\begin{array}{r}0.0 \\ 0.00 \\ \hline .00\end{array}$

$\frac{0.00}{0.00}$

$\begin{array}{r}0.0 \\ 0.00 \\ \hline 0.00\end{array}$

$\frac{0.00}{0.00}$

0.00

0.00

1.6
3.56

$\frac{3.56}{2.37}$

1.19

0.00

138,782

430,314

$5,651,356$

$16,724,223$

(1) Supplies Hermiston cogeneration facility.

2. Supplies are resold to Dynegy Marketing \& Trade, primarily for markets in California.

(4) Gas delivered to Georgia-Pacific's Plattsburg. N.Y. plant. 


\section{VOLUME AND PRICE REPORT}

\section{Long-Term Imports.}

Volumes (MMcf/d) \& Prices (\$/MMBTU) of Natural

Gas Imported During the Past 12 Months 10/01/99 - 09/30/00

Long-Term Importer

CITY OF DULUTH, MINNESOTA

(ProGas Limited)

AVG Daily Quantity

Total Price (Noyes, Minnesota)

Commodity Component

Demand Component

Reservation Fee Component

CITY OF GLENDALE, CALIFORNIA

(Engage Energy Canada, L.P.)

AVG Daily Quantity

Total Price (Eastport, ldaho)

Commodity Component

Demand Component

Reservation Fee Component

\section{CITY OF PASADENA, CALIFORNIA}

(Engage Energy Canada)

AVG Dally Quantity

Total Price (Eastport, Idaho)

Commodity Component

Demand Component

Reservation Fee Component

\section{COENERGY TRADING COMPANY}

(Various Suppliers)

AVG Daily Quantity

Total Price (Pittsburg. NH)

Commodity Component

Demand Component

Reservation Fee Component

COLONIAL NATURAL GAS

(Alberta NE Gas (TCGS Ltd. 1))

AVG Daily Quantity

Total Price (Waddington, NY)

Commodity Component

Demand Component

Reservation Fee Component
Auth.

Vols.

6.1

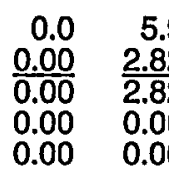

4.1

$\begin{array}{rrr}4.1 & 4.3 & 4 \\ \frac{1.91}{1.64} & \frac{1.91}{1.64} & \frac{1.9}{1.6} \\ 0.27 & 0.27 & 0.27 \\ 0.00 & 0.00 & 0.00\end{array}$

4.1

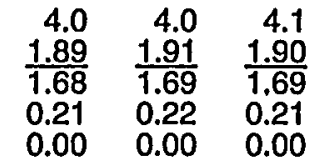

$\begin{array}{r}4.1 \\ 1.93 \\ \hline 1.69\end{array}$

$\frac{1.93}{1.69}$

0.24

0.00

80.0

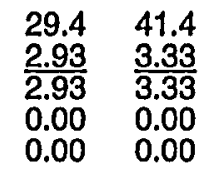

\begin{tabular}{l}
60.8 \\
2.27 \\
\hline 2.27 \\
0.00 \\
0.00
\end{tabular}

68.2

$\frac{3.61}{3.61}$

3.61
0.00

$\begin{array}{ll}3.61 & 3.46 \\ 0.00 & 0.00\end{array}$

0.00

6.0

\begin{tabular}{rr}
6.0 & 3.8 \\
2.80 & 3.22 \\
\hline 2.01 & 2.43 \\
0.79 & 0.79 \\
0.00 & 0.00
\end{tabular}

$1.64 \quad 1.64$

$\begin{array}{ll}0.31 & 0.32 \\ 0.00 & 0.00\end{array}$

0.25
0.00

0.00
Oct. Nov. Dec.

\begin{tabular}{ll}
2000 & 2000 \\
Mar. & Apr. \\
\hline
\end{tabular}
2000

20002000

\begin{tabular}{ccc}
2000 & 2000 & 2000 \\
Jul. & Aug. & Sep. \\
\hline
\end{tabular}

\begin{tabular}{rr}
6.2 & 6.2 \\
2.08 & 2.39 \\
\hline 2.08 & 2.39 \\
0.00 & 0.00 \\
.00 & 0.00
\end{tabular}

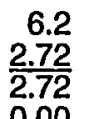

6.2
2.85
2.85
0.00

$\begin{array}{r}6.1 \\ 3.43 \\ \hline 3.43\end{array}$

$\begin{array}{r}6.3 \\ 3.88 \\ \hline\end{array}$

$\begin{array}{r}6.3 \\ 3.45 \\ \hline\end{array}$

$0.00 \quad 0.00$

$0.00 \quad 0.00$

\begin{tabular}{rrr}
4.1 & 4.1 & 4.0 \\
2.73 & 2.74 & 2.75 \\
\hline 2.39 & 2.39 & 2.39
\end{tabular}

$\frac{2.73}{2.39}$

2.39

$\frac{1.95}{1.64}$

0.31
0.00

0.31
0.00

$\frac{1.94}{1.64}$

0.30
0.00

0.31

0.34

0.00
$4.0 \quad 4.0$

$\frac{1.93}{1.68}$

$\frac{1.93}{1.69}$

4.0

4.0
190

4.0
1.92
1.67

$\begin{array}{r}4.1 \\ 2.68 \\ \hline 2.44\end{array}$

0.24
0.00

1.68
0.24

1.66

0.24
0.00

1.67

0.25
0.00

2.44

0.24
0.00

3.9

$\frac{2.69}{2.44}$

0.25
0.00

$\frac{2.70}{2.45}$

0.00

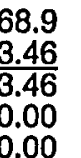

41.9
3.17
3.17
0.00
0.00

\begin{tabular}{r}
8.0 \\
3.30 \\
\hline 3.30 \\
0.00 \\
0.00
\end{tabular}

0.0
0.00
0.00
0.00
0.00

\begin{tabular}{r}
0.0 \\
0.00 \\
\hline 0.00 \\
0.00 \\
0.00
\end{tabular}

\begin{tabular}{r}
0.6 \\
4.31 \\
\hline 4.31 \\
0.00 \\
0.00
\end{tabular}

$\begin{array}{r}0.2 \\ 4.68 \\ \hline\end{array}$

4.68

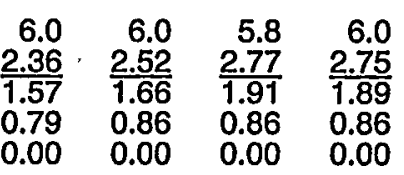

$\begin{array}{rr}6.0 & 6.0 \\ \frac{3.11}{2.25}: & \frac{3.30}{2.46} \\ 0.86 & 0.84 \\ 0.00 & 0.00\end{array}$

6.0
4.42
3.57
0.85
0.00

5.0
4.72
3.87
0.85

6.0
3.93
3.08
0.85

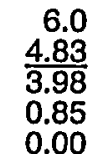

3Q 2000

OTALMCf

576,543

$\begin{array}{r}6.3 \\ 3.74 \\ \hline\end{array}$

$\frac{3.74}{3.74}$

0.00

0.00

4.0

0.25

0.00
0.00

98,461

367,155

$1,101,191$

522,000

YTD

TOTAL MC

\section{$1,697,330$}

372,638

$1,108,336$

$5,748,830$

\begin{tabular}{r}
6.0 \\
4.83 \\
\hline 3.98 \\
0.85 \\
0.00
\end{tabular}


Long-Term Importer

\section{COMMONWEALTH GAS COMPANY}

(Alberta NE Gas (TCGS Ltd. 1))

AVG Daily Quantity

Total Price (Waddington, NY)

Commodity Component

Demand Component

Reservation Fee Component

\section{CONNECTICUT NATURAL GAS}

(Álberta NE Gas (AEC Oil \& Gas Company))

AVG Daily Quantity

Total Price (Waddington, NY)

Commodity Component

Demand Component

Reservation Fee Component

CONNECTICUT NATURAL GAS

(Alberta NE Gas (TCGS Lid. 1))

AVG Daily Quantily

Total Price (Waddington, NY)

Commodity Component

Demand Component

Reservation Fee Componen

\section{CONNECTICUT NATURAL GAS}

(Alberta NE Gas (TCGS Ltd. 2))

AVG Daily Quantity

Total Price (Waddington, NY)

Commodity Component

Demand Component

Reservation Fee Component

CONNECTICUT NATURAL GAS

(Alberta Northeast Gas (ProGas))

AVG Daily Quantity

Total Price (Waddington, NY)

Commodity Component

Demand Component

Reservation Fee Component
VOLUME AND PRICE REPORT

\section{Long-Term Imports}

Volumes (MMct/d) \& Prices (\$MMBTU) of Natural

Gas Imported During the Past 12 Months 10/01/99 - 09/30/00

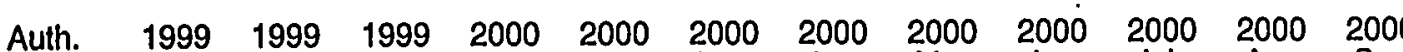

Vols. Oct. Nov. Dec. Jan. Feb. Mar. Apr. May. Jun. Jul. Aug. Sep.

$3 Q 2000$

TOTAL MC

YTD

OTAL MCf

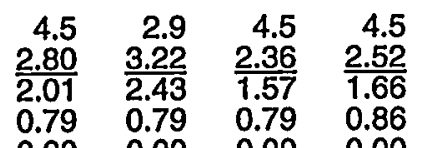

$\begin{array}{rr}4.3 & 4.5 \\ 2.77 & 2.75\end{array}$

$\frac{2.77}{1.91} \quad \frac{2.75}{1.89}$

0.860 .86

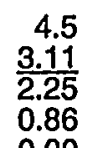

$\begin{array}{r}4.5 \\ 3.30 \\ \hline\end{array}$

$\frac{3.30}{2.46}$

$0.00 \quad 0.00$

$0.00 \quad 0.00$

0.00

0.00

\subsection{42}

3.8
4.72

$\begin{array}{llll}0.85 & 0.85 & 0.85 & 0.85\end{array}$

$\begin{array}{llll}0.00 & 0.00 & 0.00 & 0.00\end{array}$

1.2

\begin{tabular}{rrrr}
1.2 & 0.8 & 1.2 & 1.2 \\
2.94 & 3.34 & 2.67 & 2.97 \\
\hline 2.06 & $\frac{2.46}{2.46}$ & 1.79 & 2.03 \\
0.88 & 0.88 & 0.88 & 0.94 \\
0.00 & 0.00 & 0.00 & 0.00
\end{tabular}

\begin{tabular}{r}
1.2 \\
3.32 \\
\hline 2.38 \\
0.94
\end{tabular}

0.0
0.00
0.00

$\begin{array}{r}0.0 \\ 0.00 \\ \hline 0.00\end{array}$

2.94
0.94

0.00

0.00

0.00

$\begin{array}{lll}0.00 & 0.00 & 0.92\end{array}$

\begin{tabular}{l}
1.2 \\
3.57 \\
\hline 2.65 \\
0.92 \\
0.00
\end{tabular}

\begin{tabular}{r}
1.2 \\
4.72 \\
\hline 3.79 \\
0.93
\end{tabular}

$\frac{1.2}{3.71}$

$\begin{array}{lll}0.93 & 0.92 & 0.92\end{array}$

$\begin{array}{llll}0.93 & 0.93 & 0.92 & 0.92 \\ 0.00 & 0.00 & 0.00 & 0.00\end{array}$

11.5

\begin{tabular}{|c|c|c|}
\hline $\begin{array}{l}11.5 \\
2.80 \\
2.01 \\
0.79\end{array}$ & $\begin{array}{r}7.3 \\
3.22 \\
2.43 \\
0.79 \\
0.00\end{array}$ & $\begin{array}{l}11.5 \\
2.36 \\
1.57 \\
0.79 \\
0.00\end{array}$ \\
\hline
\end{tabular}

11.5
2.77
1.91
0.86
0.00

\begin{tabular}{l}
11.5 \\
2.75 \\
\hline 1.89 \\
0.86
\end{tabular}

\begin{tabular}{l}
11.5 \\
3.11 \\
\hline 2.25 \\
0.86
\end{tabular}

11.5

$\begin{array}{r}11.5 \\ 4.42 \\ \hline\end{array}$

$\frac{3.30}{2.46}$

2.46
0.84

0.00

0.00

5.3

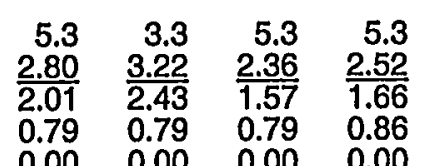

5.3
$\frac{2.77}{1.91}$

$\begin{array}{ll}1.91 & 1.89 \\ 0.86 & 0.86\end{array}$

\begin{tabular}{r}
5.3 \\
2.75 \\
\hline 1.89 \\
0.86 \\
0.00
\end{tabular}

\begin{tabular}{r}
5.3 \\
3.11 \\
\hline 2.25 \\
0.86
\end{tabular}

$\begin{array}{r}5.3 \\ .3 .30 \\ \hline\end{array}$

- $\frac{3.30}{2,46}$

0.84
0.00

4.42
3.57
0.85

0.85
0.00

10.8

3.57

0.85
0.00

4.6

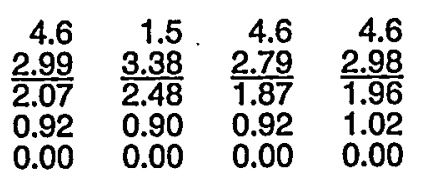

4.6
$\frac{3.42}{2.40}$
1.02
0.00

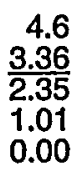

\begin{tabular}{r}
4.6 \\
3.49 \\
\hline 2.47 \\
1.02 \\
0.00
\end{tabular}

\begin{tabular}{r}
4.6 \\
3.61 \\
\hline 2.61 \\
1.00 \\
0.00
\end{tabular}

4.6

$\frac{4.70}{3.69}$

1.01

1.01
0.00 $\begin{array}{r}4.5 \\ 3.93 \\ \hline 3.08\end{array}$

4.5

$\frac{4.83}{3.98}$

$\begin{array}{r}1.2 \\ 4.33 \\ \hline 3.41\end{array}$

$\begin{array}{r}1.2 \\ 4.92 \\ \hline\end{array}$

4.00

11.5

11.5

3.87

0.85
0.00

3.93

0.85
0.00

$\frac{4.83}{3.98}$

3.98

0.85
0.00

4.9
4.72

$\frac{4.72}{3.87}$

0.85

5.3

$\frac{3.93}{3.08}$

$\begin{array}{ll}0.85 & 0.85 \\ 0.00 & 0.00\end{array}$

5.3

3.83

472,500

391,500

$1,206,000$

111,540

257,466

$1,034,996$

$3,127,996$

415,800

$1,256,640$ 


\section{VOLUME AND PRICE REPORT \\ Long-Term Imports}

Volumes (MMcf/d) \& Prices (\$MMBTU) of Natural

Gas Imported During the Past 12 Months 10/01/99 - 09/30/00

Auth

Vols.
Long-Term Importer

CONNECTICUT NATURAL GAS

(Alberta Northeast Gas (Producers Marketing)) AVG Daily Quantity

Total Price (Waddington, NY)

Commodity Component

Demand Component

Reservation Fee Component

CONSOLIDATED EDISON COMPANY OF N.Y.

(Alberta NE Gas (AEC Oil \& Gas))

AVG Daily Quantity

Total Price (Waddington, NY)

Commodity Component

Demand Component

Reservation Fee Component

CONSOLIDATED EDISON COMPANY OF N.Y.

(Alberta NE Gas (ProGas))

AVG Daily Guantity

Total Price (Waddington, NY)

Commodity Component

Demand Component

Reservation Fee Component

Oct. Nov

2.4

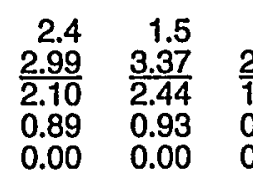

1.1

\begin{tabular}{lr}
1.1 & 0.5 \\
$\frac{2.94}{2.06}$ & $\frac{3.34}{2.46}$ \\
\hline 0.88 & .0 .88
\end{tabular}

$\begin{array}{ll}0.88 & 0.88 \\ 0.00 & 0.00\end{array}$

5.3

$\begin{array}{rrrr}5.3 & 1.8 & 5.3 & 5.3 \\ \frac{2.99}{2.07} & \frac{3.38}{2.48} & \frac{2.79}{1.87} & \frac{2.98}{1.96} \\ 0.92 & 0.90 & 0.92 & 1.02 \\ 0.00 & 0.00 & 0.00 & 0.00\end{array}$

(Alberta NE Gas (Producers Marketing))

AVG Daily Quantity

Total Price (Waddington, NY)

Commodity Componen

Reservation Fee Component

CONSOLIDATED EDISON COMPANY OF N.Y.

(Alberta NE Gas (TCGS Ltd. 1))

AVG Daily Quantity

Total Price (Waddington, NY)

Commodity Component

Demand Component

Reservation Fee Component

\begin{tabular}{rr}
2.4 & 2.4 \\
79 & $\frac{2.98}{1.99}$ \\
\hline 90 & 0.99
\end{tabular}

1.1
. .67
0.88
0.00

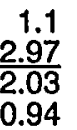

2.6

$\begin{array}{rrrrrrrrrrrr}2.6 & 1.8 & 2.6 & 2.6 & 2.6 & 2.6 & 2.6 & 2.6 & 2.6 & 2.6 & 2.6 & 2.6 \\ \frac{2.99}{2.10} & \frac{3.37}{2.44} & \frac{2.79}{1.90} & \frac{2.98}{1.99} & \frac{3.42}{2.44} & \frac{3.37}{2.39} & \frac{3.49}{2.51} & \frac{.3 .60}{2.64} & \frac{3.69}{2.72} & \frac{4.82}{3.94} & \frac{4.78}{3.91} & \frac{4.20}{3.30} \\ 0.89 & 0.93 & 0.89 & 0.99 & 0.98 & 0.98 & 0.98 & 0.96 & 0.97 & 0.88 & 0.87 & 0.90 \\ 0.00 & 0.00 & 0.00 & 0.00 & 0.00 & 0.00 & 0.00 & 0.00 & 0.00 & 0.00 & 0.00 & 0.00\end{array}$

0.00

0.00

0.00

0.00

0.00

.
2000

$\begin{array}{ll}2000 & 2000 \\ \text { Mar. } & \text { Apr. }\end{array}$

Apr.

May.

$\frac{3.42}{2.44}$

$\frac{3.47}{2.39}$

$\begin{array}{r}2.4 \\ 3.49 \\ \hline 2.51\end{array}$

2.4
$\frac{3.60}{2.64}$

$0.98 \quad 0.96$

0.00

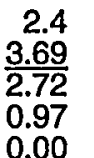

$\begin{array}{r}2.3 \\ 4.75 \\ \hline 3.78\end{array}$

0.97
0.00

4.33
3.36

3.36
0.97
0.00

5.06

4.09

0.97
0.00

0.00
1.1
3.32
2.38

$\begin{array}{r}0.0 \\ 0.00 \\ \hline .00\end{array}$

$\frac{0.00}{0.00} \quad \frac{0.00}{0.00}$

$\frac{3.1 .1}{2.65}$

1.1
4.72
3.79

1.1
4.71
3.78

1.1
$\frac{4.33}{3.41}$

$\begin{array}{r}1.1 \\ 4.92 \\ \hline\end{array}$

4.00

$\begin{array}{lll}0.00 & 0.00 & 0.00\end{array}$

0.92

0.00

0.93

$0.00 \quad 0.92$

102,028

236,217

485,760

$1,446,720$

$\frac{3.42}{2.40}$

1.02

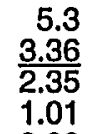

$\begin{array}{r}5.3 \\ 3.49 \\ \hline 2.47\end{array}$

1.02

5.3
$\frac{3.61}{2.61}$

1.00

$\begin{array}{r}5.3 \\ 4.70 \\ \hline 3.69\end{array}$

3.69
1.01

5.3
3.75

3.75
1.01

$\begin{array}{r}5.3 \\ 4.33 \\ \hline 3.33\end{array}$

1.00

$\begin{array}{r}5.3 \\ 5.05 \\ \hline .05\end{array}$

1.00
0.00

240,212

715,414

$\begin{array}{rrrr}5.0 & 3.2 & 5.0 & 5.0 \\ \frac{2.80}{2.01} & \frac{3.22}{2.43} & \frac{2.36}{1.57} & \frac{2.52}{1.66} \\ 0.79 & 0.79 & 0.79 & 0.86 \\ 0.00 & 0.00 & 0.00 & 0.00\end{array}$

\begin{tabular}{r}
5.0 \\
2.77 \\
\hline 1.91 \\
0.86
\end{tabular}

\begin{tabular}{r}
5.0 \\
2.75 \\
\hline 1.89 \\
0.86 \\
0.00
\end{tabular}

5.0
$\frac{3.11}{2.25}$
0.86

\begin{tabular}{r}
5.0 \\
3.30 \\
\hline 2.46 \\
0.84
\end{tabular}

5.0
4.42
3.57
0.85

4.2
$\frac{4.72}{3.87}$
0.85

5.0
$\frac{3.93}{3.08}$
0.85

$\begin{array}{r}5.0 \\ 4.83 \\ \hline\end{array}$

3.98

0.85

0.00
435,000

$1,345,000$ 


\section{VOLUME AND PRICE REPORT}

\section{Long-Term Imports}

Volumes (MMcf/d) \& Prices (\$MMBTU) of Natural Gas Imported During the Past 12 Months 10/01/99 - 09/30/00

Long-Term Importer

$\begin{array}{lllllllllllll}\text { Auth. } & 1999 & 1999 & 1999 & 2000 & 2000 & 2000 & 2000 & 2000 & 2000 & 2000 & 2000 & 2000\end{array}$

Vols.

Oct. Nov.

Dec.

Jan.

2000

Apr.

$\begin{array}{rrrr}6.0 & 3.8 & 6.0 & 6.0 \\ \frac{2.80}{2.01} & \frac{3.22}{2.43} & \frac{2.36}{1.57} & \frac{2.52}{1.66} \\ 0.79 & 0.79 & 0.79 & 0.86 \\ 0.00 & 0.00 & 0.00 & 0.00\end{array}$

$\begin{array}{rr}6.0 & \\ \frac{2.77}{1.91} & 2.0 \\ 0.86 & 0.80 \\ 0.00 & 0.00\end{array}$

\begin{tabular}{rr}
6.0 & 6.0 \\
2.75 & 3.11 \\
\hline 1.89 & 2.25 \\
0.86 & 0.86 \\
0.00 & 0.00
\end{tabular}

\begin{tabular}{r}
6.0 \\
3.30 \\
\hline 2.46 \\
0.84
\end{tabular}

6.0
4.4
3.5
0.8
0.00

CORAL ENERGY RESOURCES, L.P.-ENRON GAS MARKETING (1)

(Shell Canada Limited)
AVG Daily Quantity

9.8

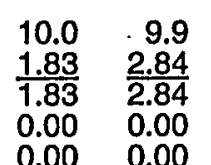

9.7
$\frac{2.12}{2.12}$
0.00
0.00

9.9
2.14
2.14
0.00
0.00

10.0
2.16
2.16
0.00
0.00

\begin{tabular}{r}
9.9 \\
2.09 \\
\hline 2.09 \\
0.00 \\
0.00
\end{tabular}

10.0
2.38
2.38

0.00

0.00

$0.00 \cdot 0.00$

$\begin{array}{ll}0.00 & 0.00\end{array}$

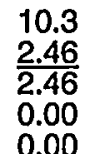

9.9
3.49
3.49

Reservation Fee Component

20.5

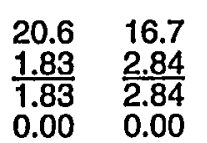

$0.00 \quad 0.00$

$0.00 \quad 0.00$
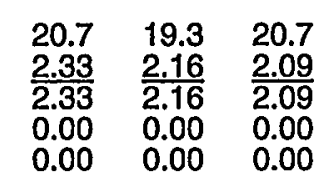

20.7
2.80
2.80
0.00

Reservation Fee Component

\section{CRESTAR ENERGY MARKETING CORPORATION (3)}

(Crestar Energy) AVG Daily Quantity

Total Price (Noyes, Minnesota)

15.0

\begin{tabular}{llll}
14.2 & 10.6 & 14.6 & 14.8 \\
2.75 & 3.57 & 2.44 & 2.62 \\
\hline 2.14 & $\frac{2.74}{1.87}$ & $\frac{2.04}{2.74}$ \\
0.52 & 0.68 & 0.49 & 0.50 \\
0.09 & 0.15 & 0.08 & 0.08
\end{tabular}

\begin{tabular}{l}
13.8 \\
2.95 \\
\hline 2.29 \\
0.56 \\
0.10
\end{tabular}
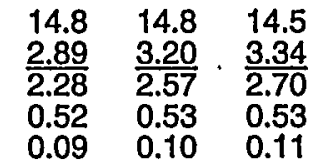

\begin{tabular}{l}
14.8 \\
4.63 \\
\hline 3.96 \\
0.55 \\
0.12
\end{tabular}

11.6
4.82
4.00
0.67

\begin{tabular}{ll}
1.6 & 13.3 \\
\hline .02 & 4.22 \\
\hline & 3.50 \\
07 & 0.59
\end{tabular}

$\frac{4.22}{3.50}$

14.2

$\frac{4.99}{4.28}$

Reservation Fee Component

(i) Successor to Salmon Resources Ltd. (See DOE/FE order 690-A).

(3) Supply is sold to Northern States Power Company for retall customers in the Grand Forks and Fargo areas of North Dakota. 
VOLUME AND PRICE REPORT

Long-Term Imports

Volumes (MMct/d) \& Prices (\$MMBTU) of Natural Gas Imported During the Past 12 Months 10/01/99 - 09/30/00

Auth. $\quad \begin{array}{lllllllllll}1999 & 1999 & 1999 & 2000 & 2000 \cdot 2000 & 2000 & 2000 & 2000 & 2000 & 2000 & 2000\end{array}$ Vols. Oct. Nov. Dec. Jan. Feb. Mar. Apr. May. Jun. Jul. Aug. Sep.

Long-Term Importer

CRESTAR ENERGY MARKETING CORPORATION (1) (Crestar Energy)

Total Price (Port of Morgan, MT)

6.3

Commodity Componen

Demand Component

Reservation Fee Component

DARTMOUTH POWER ASSOCIATES, L.P

(Anderson Exploration Ltd.)

Total Price (Waddinglon, NY)

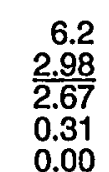

\begin{tabular}{r}
6.3 \\
4.33 \\
\hline 4.01 \\
0.32
\end{tabular}

\begin{tabular}{r}
5.3 \\
4.35 \\
\hline 4.02 \\
0.33
\end{tabular}

$\begin{array}{r}6.3 \\ 3.88 \\ \hline 3.56\end{array}$

$\frac{3.88}{3.56}$

0.32

4.66

4.34

0.32
AVG Daily Quantity

Total Price (Waddington, NY)

Commodity Componen

Demand Component

Reservation Fee Component

DARTMOUTH POWER ASSOCIATES, L.P.

(Crestar Energy Inc.)

AVG Daily Quantity

Total Price (Waddington, NY)

Commodity Component

Demand Component

Reservation Fee Component

DARTMOUTH POWER ASSOCIATES, L.P. (2)

(Direct Energy Marketing Ltd.)

AVG Daily Quantity

Commodity Component

Demand Component

Reservation Fee Component

\begin{tabular}{rrr}
0.0 & 0.0 & 0.0 \\
0.00 & 0.00 & 0.00 \\
\hline 0.00 & 0.00 & 0.00 \\
0.00 & 0.00 & 0.00 \\
0.00 & 0.00 & 0.00
\end{tabular}

$\begin{array}{rr}0.0 & 0.0 \\ 0.00 & \frac{0.00}{0.00}\end{array}$

$\begin{array}{ll}0.00 & 0.00 \\ 0.00 & 0.00\end{array}$

$0.00 \quad 0.00$

0.00

0.00

\begin{tabular}{r}
0.0 \\
0.00 \\
\hline 0.00 \\
0.00
\end{tabular}

0.00

0.00

0.00

0.00
0.00

DARTMOUTH POWER ASSOCIATES, L.P.

(Remington Energy Ltd.)

AVG Daily Quantity

3.0

Total Price (Waddington, NY)

Commodity Componen

Reservation Fee Component
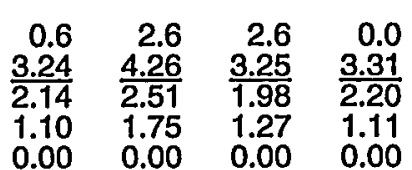

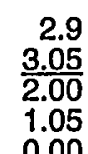

\begin{tabular}{r}
2.8 \\
3.11 \\
\hline 2.07 \\
1.04 \\
0.00
\end{tabular}

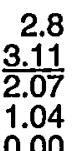

\begin{tabular}{r}
2.8 \\
3.30 \\
\hline 2.25 \\
1.05 \\
0.00
\end{tabular}

0.00

$\begin{array}{rrrrr}9.3 & 9.7 & 9.6 & 9.3 & 5.7 \\ \frac{3.43}{2.41} & \frac{4.31}{3.11} & \frac{4.17}{3.10} & \frac{4.33}{3.27} & \frac{7.49}{6.17} \\ 1.02 & 1.20 & 1.07 & 1.06 & 1.32 \\ 0.00 & 0.00 & 0.00 & 0.00 & 0.00\end{array}$

1.8

4.97

3.65

1.32

0.00

$\begin{array}{llll}1.02 & 1.20 & 1.07 & 1.06\end{array}$

$\begin{array}{lll}0.00 & 0.00 & 0.00\end{array}$

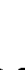

0.00

0.00

0.0
0.00

0.00

$\begin{array}{r}0.0 \\ 0.00 \\ \hline 0.00\end{array}$

$\frac{0.00}{0.00}$

0.0
0.00

$\frac{0.00}{0.00}$

$\begin{array}{r}0.0 \\ 0.00 \\ \hline 0.00\end{array}$

0.00
0.00

$\begin{array}{llll}0.00 & 0.00 & 0.00 & 0.00 \\ 0.00 & 0.00 & 0.00 & 0.00\end{array}$

(1) Supply is sold to Northern States Power Company (Minnesota).
(2) Direct Energy Marketing Ltd., as agent for Dartmouth, on occasion will supply natural gas for balancing purposes.

\section{$3 Q 2000 \quad$ YTD TOTAL MCf TOTALMCf}

552,899

$1,681,975$

757,951

$2,546,381$

164,707

324,062

124,800

465,524 


\section{VOLUME AND PRICE REPORT}

\section{Long-Term Imports}

Volumes (MMcf/d) \& Prices (\$/MMBTU) of Natural Gas Imported During the Past 12 Months 10/01/99 - 09/30/00
Long-Term Importer

DISTRIGAS CORPORATION (88-37-LNG) (1)

(Sonatrach)

AVG Daily Quantity

Total Price (Everett, Massachusetts)

Commodity Component

Demand Component

Reservation Fee Component

DISTRIGAS CORPORATION (89-16-LNG) (2)

(Sonatrach)

AVG Daily Quantity

Total Price (Everett, Massachusetts)

Commodity Component

Demand Component

Reservation Fee Component

DISTRIGAS CORPORATION (95-100-LNG) (3)

(Cabot LNG Trading Limited)

AVG Daily Quantity

Total Price (Everett, Massachusetts)

Commodity Component

Demand Component

Reservation Fee Component

DUKE ENERGY LNG SALES, INC. (4)

(Sonatrading)

AVG Daily Quantity

Total Price (Lake Charles, LA)

Commodity Componen

Demand Component

Reservation Fee Component

\section{ECOELECTRICA, L.P.}

(Cabot LNG Trading Limited)

AVG Daily Quantity

Total Price (Ponce, Puerto Rico)

Commodity Component

Demand Component

Reservation Fee Component

\section{A}

Auth

$\begin{array}{lllll}1999 & 1999 & 1999 & 2000\end{array}$

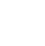

$\begin{array}{rrrrrrrrrrrr}80.7 & 81.3 & 80.4 & \mathbf{8 1 . 3} & 172.0 & 46.5 & 0.0 & 0.0 & 0.0 & 0.0 & 76.4 & 0.0 \\ \frac{2.58}{2.58} & \frac{2.63}{2.63} & \frac{2.63}{2.63} & \frac{2.63}{2.63} & \frac{\mathbf{3 . 3 4}}{3.34} & \frac{2.59}{2.59} & \frac{0.00}{0.00} & \frac{0.00}{0.00} & \frac{0.00}{0.00} & \frac{0.00}{0.00} & \frac{3.44}{3.44} & \underline{0.00} \\ 0.00 & 0.00 & 0.00 & 0.00 & 0.00 & 0.00 & 0.00 & 0.00 & 0.00 & 0.00 & 0.00 & 0.00 \\ 0.00 & 0.00 & 0.00 & 0.00 & 0.00 & 0.00 & 0.00 & 0.00 & 0.00 & 0.00 & 0.00 & 0.00\end{array}$

0.0

\begin{tabular}{rrrrr}
0.0 & 0.0 & 0.0 & 0.0 & 0.0 \\
$\underline{0.00}$ & 0.00 & 0.00 & 0.00 & 0.00 \\
\hline 0.00 & 0.00 & 0.00 & $\overline{0.00}$ & 0.00 \\
0.00 & 0.00 & 0.00 & 0.00 & 0.00 \\
0.00 & 0.00 & 0.00 & 0.00 & 0.00
\end{tabular}

0.0
0.00
0.00
0.00
0.00

0.0
0.00
0.00
0.00
0.00

0.0

$\frac{0.00}{0.00}$

0.00

0.00
0.00

\begin{tabular}{r}
0.0 \\
0.00 \\
\hline 0.00 \\
0.00
\end{tabular}

0.00
0.00
0.00

0.00

0.0

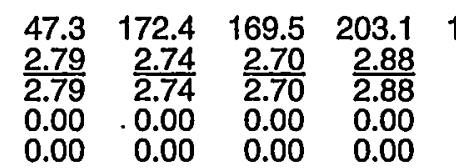

\begin{tabular}{r}
178.2 \\
2.77 \\
\hline 2.77 \\
0.00 \\
0.00
\end{tabular}

\begin{tabular}{r}
155.0 \\
2.73 \\
\hline 2.73 \\
0.00 \\
0.00
\end{tabular}

\begin{tabular}{rr}
221.2 & 24 \\
2.93 & 2 \\
\hline 2.93 & 2.00 \\
0.00 & 0 \\
0.00 & 0
\end{tabular}

249.6
2.87

2.87
2.87
0.00
0.00

$\begin{array}{llll}97.9 & 262.1 & 213.9 & 162.1\end{array}$

$\frac{3.14}{3.14}$

3.14
0.00

0.00
0.00

2.99
2.99
0.00
0.00

2.96
2.96
0.00

$96 \quad 2.84$

$0.00 \quad 0.00$

123.3

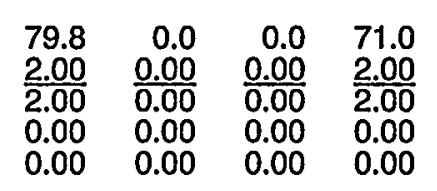

\begin{tabular}{r}
0.0 \\
0.00 \\
\hline 0.00 \\
0.00 \\
0.00
\end{tabular}

\begin{tabular}{l}
76.0 \\
2.00 \\
\hline 2.00 \\
0.00
\end{tabular}

\begin{tabular}{l}
72.7 \\
2.42 \\
\hline 2.42 \\
0.00
\end{tabular}

70.5
.2 .78
2.78
0.00

\begin{tabular}{l}
74.5 \\
3.14 \\
\hline 3.14
\end{tabular}

$\frac{3.14}{3.14}$

3.14
0.00
0.00

71.2
3.02
3.02
0.00
0.00

0.00

0.00

0.0

\begin{tabular}{rrrr}
0.0 & 0.0 & 0.0 & 0.0 \\
$\underline{0.00}$ & $\underline{0.00}$ & $\underline{0.00}$ & $\underline{0.00}$ \\
\hline 0.00 & 0.00 & 0.00 & 0.00 \\
0.00 & 0.00 & 0.00 & 0.00
\end{tabular}

0.0
0.00
0.00
0.00

0.0
0.00
0.00
0.00

\begin{tabular}{r}
0.0 \\
0.00 \\
\hline 0.00 \\
0.00
\end{tabular}

\begin{tabular}{rr}
0.0 & 0.0 \\
0.00 & 0.00 \\
\hline 0.00 & 0.00 \\
0.00 & 0.00
\end{tabular}

$\begin{array}{llll}0.00 & 0.00 & 0.00 & 0.00\end{array}$ 


\section{VOLUME AND PRICE REPORT}

\section{Long-Term Imports}

Volumes (MMcf/d) \& Prices (\$/MMBTU) of Natura Gas Imported During the Past 12 Months 10/01/99 - 09/30/00

Long-Term Importer

\section{ELIZABETHTOWN GAS COMPANY}

(TCGS Ltd.)

AVG Daily Quantity

Total Price (Niagara Falls, NY)

Commodity Component

Demand Component

Reservation Fee Component

ENCOGEN NORTHWEST, L.P. (1)

(CanWest Gas Supply, Inc.)

AVG Daily Quantity

Total Price (Sumas, Washington)

Commodity Componen

Demand Component

Reservation Fee Component

ENERGYNORTH, INC.

(Alberta NE Gas (TCGS Ltd. 1))

AVG Daily Quantity

Total Price (Waddington. NY)

Commodity Componen

Demand Component

Reservation Fee Component

ENGAGE ENERGY CANADA LP. - CIBOLA CORP.

(Engage Energy Canada, L.P.)

AVG Daily Quantity

Total Price (Port of Morgan, MT)

Commodity Component

Demand Component

Auth. $\quad 1999 \quad 1999 \quad 1999 \quad 2000 \quad 2000$

Vols.

Reservation Fee Component
10.

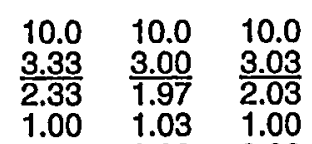

$\begin{array}{lll}1.00 & 1.03 & 1.00\end{array}$

$\begin{array}{llll}0.00 & 0.00 & 0.00 & 0.00\end{array}$

9.6

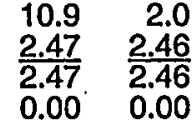

$0.00 \quad 0.00$

$0.00 \quad 0.00$

4.0

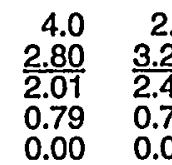

1.2

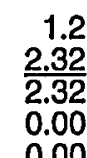

1.2
2.89
0.00
0.00 \begin{tabular}{rr}
0.3 & 0.0 \\
2.17 & 0.00 \\
\hline 2.17 & 0.00 \\
0.00 & 0.00
\end{tabular}

0.00

0.00

\begin{tabular}{ll}
2.9 & 4.0 \\
.22 & 2.36 \\
\hline .79 & 0.79 \\
.00 & 0.00
\end{tabular}

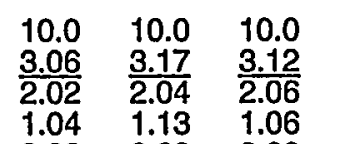

$\begin{array}{lll}1.04 & 1.13 & 1.06\end{array}$
2000

2000

pr.
20002

May. Jum

\section{ENGAGE ENERGY CANADA, L.P. - I'STATE POWER CO. (2)}

(Engage Energy Canada, L.P.)

AVG Daily Quantity

Total Price (Port of Morgan, MT)

1.0

\begin{tabular}{rrr}
1.1 & 0.0 & 0.0 \\
2.35 & 0.00 & 0.00 \\
\hline 2.08 & 0.00 & 0.00 \\
0.27 & 0.00 & 0.00 \\
0.00 & 0.00 & 0.00
\end{tabular}

Demand Compont

Reservation Fee Component .

$\begin{array}{rrrrr}4.0 & 4.0 & 3.9 & 4.0 & 4.0 \\ 2.36 & 2.52 & \frac{2.77}{1.91} & \frac{2.75}{1.89} & \frac{3.11}{2.25} \\ 1.57 & \frac{1.66}{1.99} & 0.86 & 0.86 & 0.86 \\ 0.79 & 0.86 & 0.80 & 0.00 & 0.00\end{array}$

\begin{tabular}{r}
4.0 \\
3.30 \\
\hline 2.46 \\
0.84
\end{tabular}

4.0
4.42
3.57

3.57

0.85

3.4
4.72
3.87
0.85
0.00

$\frac{3.93}{3.08}$

3.08

0.85

0.00

0.00

$0.00 \quad 0.00$

1) Supply used to fuel a 160 Megawatt gas-fired cogeneration faclity located at Bellingham, Washington.

(1) Cupply used to fuel a 160 Megawatt gas-fired cogeneration factily

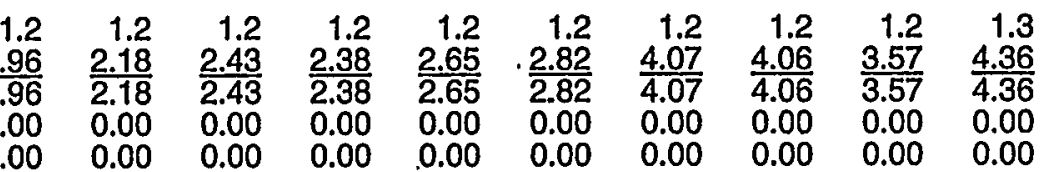

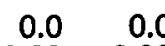

$\underline{0.00}$

$0.00 \quad 0.00$

0.00
0.00
$0.0 \quad 0.0$

$0.00 \quad 0.00$

$0.00 \quad 0.00$

$\begin{array}{llll}0.00 & 0.00 & 0.00 & 0.00\end{array}$

0.0
.00
.00
.00

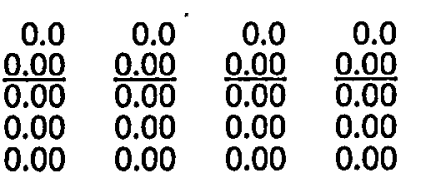

YTD

$3 Q 2000$

920,000

$2,740,000$

881,268

$1,999,934$

$\frac{2.59}{2.59}$

0.00

348,000

$1,072,000$

4.83

0.85 00 


\section{VOLUME AND PRICE REPORT}

\section{Long-Term Imports}

Volumes (MMcf/d) \& Prices (\$/MMBTU) of Natural

Gas Imported During the Past 12 Months 10/01/99 - 09/30/00

Long-Term Importer

Auth. 1999

$\begin{array}{ll}1999 & 1999 \\ \text { Oct. Nov. }\end{array}$

19992000

2000

2000

2000

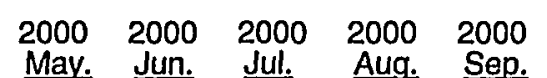

$3 Q 2000$

TOTAL MC

YTD

ENGAGE ENERGY CANADA, L.P. - METRO. UTIL. DIS

(Engage Energy Canada, L.P.)

AVG Daily Quantity

3.7

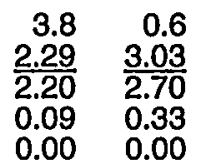

\begin{tabular}{r}
3.8 \\
1.97 \\
\hline 1.86 \\
0.11 \\
0.00
\end{tabular}

\begin{tabular}{r}
3.8 \\
2.21 \\
\hline 2.02 \\
0.19 \\
0.00
\end{tabular}

\begin{tabular}{r}
3.8 \\
2.45 \\
\hline 2.25 \\
0.20 \\
0.00
\end{tabular}

3.8
2.43
2.24
0.19
0.00

\begin{tabular}{r}
3.8 \\
2.73 \\
\hline 2.53 \\
0.20 \\
0.00
\end{tabular}

3.8

3.8

$\begin{array}{r}3.7 \\ 4.07 \\ \hline 3.87\end{array}$

Demand Component

Reservation Fee Component

ENGAGE ENERGY CANADA, L.P. - MIDAMERICAN

(Engage Energy Canada, L.P.)

AVG Daily Quantity

Total Price (Port of Morgan, MT)

6.5

Commodity Component

Demand Component

Reservation Fee Component

$\begin{array}{rrrr}6.7 & 2.7 & 6.7 & 6.7 \\ \frac{2.17}{2.08} & \frac{2.76}{2.54} & \frac{1.87}{1.78} & \frac{2.08}{1.93} \\ 0.09 & 0.22 & 0.09 & 0.15 \\ 0.00 & 0.00 & 0.00 & 0.00\end{array}$

\begin{tabular}{r}
6.7 \\
2.31 \\
\hline 2.15 \\
0.16 \\
0.00
\end{tabular}

6.7
2.28
2.13
0.15
0.00

6.7
2.56
.2 .40
0.16

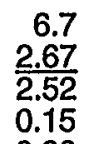

$\begin{array}{r}6.6 \\ 3.78 \\ \hline\end{array}$

0.16

0.15

3.62

0.16

$\frac{3.78}{3.62}$

0.16

$\frac{3.34}{3.19}$

ENGAGE ENERGY CANADA, L.P. - N'WESTERN PUB.

(Engage Energy Canada, L.P.)

AVG. Daily Quantity

0.9

Total Price (Port of Morgan, MT)

Commodity Componen

Demand Component

Reservation Fee Component

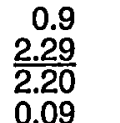

0.3
2.97
2.70
0.27

$\frac{1.0}{1.95}$

$0.27 \quad 0.09$

0.00

0.00

1.0
2.21
2.02
0.19

0.19

1.0
2.45
2.25
0.20
0.00

$\frac{2.43}{2.24}$

ENGAGE ENERGY CANADA, L.P. - WISCONSIN GAS

(Engage Energy Canada, L.P.)

AVG Daily Quantity

Total Price (Port of Morgan, MT)

2.4

Commodity Component

Demand Component

Reservation Fee Component

ENGAGE ENERGY U.S., L.P.

(Engage Energy Canada, L.P.)

AVG Daily Quantity

Total Price (Grand Island, NY)

Commodity Component

Demand Component

Reservation Fee Component

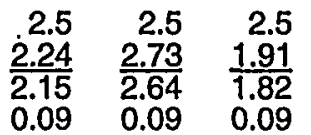

$\begin{array}{lll}0.09 & 0.09 & 0.09 \\ 0.00 & 0.00 & 0.00\end{array}$

2.5
2.14
1.98
0.16
0.00

2.5
2.36
2.20
0.16

2.5

$\frac{2.35}{2.19}$

2.19
0.16

0.16
0.00

2.63

2.47
0.16

0.00

48.3

\begin{tabular}{rrr}
8.0 & 0.0 & 0.0 \\
2.95 & 0.00 & 0.00 \\
\hline 2.95 & 0.00 & 0.00 \\
0.00 & 0.00 & 0.00 \\
0.00 & 0.00 & 0.00
\end{tabular}

\begin{tabular}{r}
0.0 \\
0.00 \\
\hline 0.00 \\
0.00 \\
0.00
\end{tabular}

0.0
0.00
0.00
0.00
0.00

\begin{tabular}{r}
0.0 \\
0.00 \\
\hline 0.00 \\
0.00 \\
0.00
\end{tabular}

0.0
0.00
0.00

$\frac{0.00}{0.00}$

$\frac{0.00}{0.00} \quad 0.0$

$\begin{array}{r}0.0 \\ 0.00 \\ \hline 0.00\end{array}$

$\frac{0.00}{0.00}$

0.00

$\begin{array}{llllllllllll}0.00 & 0.00 & 0.00 & 0.00 & 0.00 & 0.00 & 0.00 & 0.00 & 0.00 & 0.00 & 0.00 & 0.00 \\ 0.00 & 0.00 & 0.00 & 0.00 & 0.00 & 0.00 & 0.00 & 0.00 & 0.00 & 0.00 & 0.00 & 0.00\end{array}$ \begin{tabular}{r}
0.0 \\
0.00 \\
\hline 0.00 \\
0.00 \\
0.00
\end{tabular}
$346,864 \quad 1,036,730$

610,701

$1,825,404$

83,911

259,463

227,132

678,958

0

0 


\section{VOLUME AND PRICE REPORT}

\section{Long-Term Imports}

Volumes (MMcf/d) \& Prices (\$/MMBTU) of Natural

Gas Imported During the Past 12 Months 10/01/99 - 09/30/00

\section{Long-Term Importer}

ENGAGE ENERGY U.S., L.P. (1)

Morgan Hydrocarbons Inc.)

Total Price (Waddington, NY)

Commodity Component

Demand Component

Reservation Fee Component

ENGAGE ENERGY U.S., L.P. (2)

AVG Daily Quantily

Total Price (Noyes, Minnesota)

Commodity Component

Demand Component

Reservation Fee Component

ENGAGE ENERGY U.S., L.P. (3)

(Petro-Canada Resources)

AVG Daily Quantily

Total Price (Noyes, Minnesota)

Commodily Component

Demand Component

Reservation Fee Component

ENGAGE ENERGY U.S., L.P. (4)

(Rio Alto, Pinnacle, Jordan)

AVG Daily Quantity

Total Price (Waddington, NY)

Commodity Component

Demand Component

Reservation Fee Component

\section{ENGAGE ENERGY U.S., L.P.}

(Various Suppliers)

AVG Daily Quantity

Demand Component
AVG Daily Quantity

(Morrison Petroleums Ltd.)

Total Price (Noyes, Minnesota)

Commodity Componen

Reservation Fee Component
Auth.

19991999

Vols.

10.0

$\begin{array}{rllllllllllll}10.2 & 10.2 & 10.1 & 10.2 & 9.4 & 10.2 & 10.2 & 10.1 & 10.1 & 10.2 & 10.2 & 10.1 \\ \frac{2.76}{2.76} & \frac{3.42}{3.42} & \frac{2.57}{2.57} & \frac{2.83}{2.83} & \frac{3.14}{3.14} & \frac{2.96}{2.96} & \frac{3.15}{3.15} & \frac{3.37}{3.37} & \frac{4.67}{4.67} & \frac{4.70}{4.70} & \frac{4.12}{4.12} & \frac{4.93}{4.93} \\ 0.00 & 0.00 & 0.00 & 0.00 & 0.00 & 0.00 & 0.00 & 0.00 & 0.00 & 0.00 & 0.00 & 0.00 \\ 0.00 & 0.00 & 0.00 & 0.00 & 0.00 & 0.00 & 0.00 & 0.00 & 0.00 & 0.00 & 0.00 & 0.00\end{array}$

7.0

\begin{tabular}{|c|c|c|c|c|c|c|c|c|c|c|c|}
\hline $\begin{array}{r}7.1 \\
\frac{2.12}{2.12} \\
0.00\end{array}$ & $\begin{array}{r}7.2 \\
2.50 \\
2.50 \\
0.00 \\
0.00\end{array}$ & $\begin{array}{r}7.2 \\
1.76 \\
1.76 \\
0.00 \\
0.00\end{array}$ & $\begin{array}{r}7.2 \\
1.96 \\
1.96 \\
0.00 \\
0.00\end{array}$ & $\begin{array}{r}7.2 \\
2.19 \\
2.19 \\
0.00 \\
0.00\end{array}$ & $\begin{array}{r}7.2 \\
2.21 \\
2.21 \\
0.00\end{array}$ & $\begin{array}{r}7.2 \\
2.43 \\
2.43 \\
0.00 \\
0.00\end{array}$ & $\begin{array}{r}7.1 \\
\frac{2.63}{2.63}\end{array}$ & $\begin{array}{r}7.2 \\
3.84 \\
3.84 \\
0.00 \\
0.00\end{array}$ & & & 4.14 \\
\hline
\end{tabular}

\begin{tabular}{ll|}
15.2 & 15.2 \\
$\frac{2.64}{2.64}$ & $\frac{3.24}{3.24}$ \\
0.00 & 0.00 \\
0.00 & 0.00
\end{tabular}

15.2
$\frac{2.34}{2.34}$
0.00
0.00

\begin{tabular}{l}
15.2 \\
2.37 \\
\hline 2.37 \\
0.00 \\
0.00
\end{tabular}

11.1

$\begin{array}{llll}11.2 & 11.2 & 11.2 & 11.2 \\ 2.12 & 2.51 & 1.76 & \frac{1.96}{2.12} \\ \frac{2.51}{2.51} & 1.76 & 1.96 \\ 0.00 & 0.00 & 0.00 & 0.00 \\ 0.00 & 0.00 & 0.00 & 0.00\end{array}$

14.0

\begin{tabular}{rr}
9.2 & 9.2 \\
2.76 & 3.42 \\
\hline 2.76 & 3.42 \\
0.00 & 0.00
\end{tabular}

$\begin{array}{ll}0.00 & 0.00\end{array}$

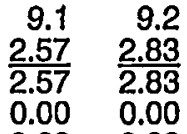

$0.00 \quad 0.00$

$0.00 \quad 0.00$

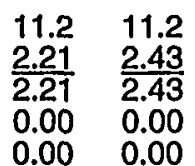

11.2
2.60
2.60
0.00
0.00

11.2
3.75
3.75
0.00
0.00

$\begin{array}{r}11.2 \\ 3.49 \\ \hline\end{array}$

$\frac{3.49}{3.49}$

0.00

11.2
$\frac{3.35}{3.35}$

3.35
0.00

2.19
0.00
0.00

0.00

9.3

3.14

3.14
0.00

$0.00 \quad 0.00$

9.2
3.15
3.15
0.00
0.00

\begin{tabular}{r}
9.1 \\
$\cdot 3.37$ \\
\hline 3.37 \\
0.00
\end{tabular}

\begin{tabular}{r}
9.1 \\
4.67 \\
\hline 4.67 \\
0.00
\end{tabular}

\begin{tabular}{r}
9.1 \\
4.70 \\
\hline 4.70 \\
0.00
\end{tabular}

$\begin{array}{r}9.5 \\ 4.14 \\ \hline 4.14\end{array}$

$\frac{4.14}{4.14}$

0.00

$\begin{array}{r}8.3 \\ 4.93 \\ \hline 4.93\end{array}$

4.93

$\begin{array}{lllll}0.00 & 0.00 & 0.00 & 0.00 & 0.00\end{array}$

\section{0}

\begin{tabular}{|c|c|c|c|c|c|}
\hline $\begin{array}{l}15.2 \\
2.70 \\
2.70 \\
0.00 \\
0.00\end{array}$ & $\begin{array}{l}15.2 \\
3.00 \\
3.00 \\
0.00 \\
0.00\end{array}$ & $\begin{array}{l}15.2 \\
3.23 \\
3.23 \\
0.00 \\
0.00\end{array}$ & $\begin{array}{l}15.2 \\
4.54 \\
4.54 \\
0.00 \\
0.00\end{array}$ & $\begin{array}{l}15.2 \\
4.45 \\
4.45 \\
0.00 \\
0.00\end{array}$ & $\begin{array}{l}15.2 \\
3.95 \\
3.95 \\
0.00 \\
0.00\end{array}$ \\
\hline
\end{tabular}

$3 Q 2000$

TOTAL MOF

933,875

$2,762,648$

660,744

$1,966,389$

$1,028,376$

$3,064,256$

824,599

$2,495,783$

(1) Supplies will serve CGM's markets in the U.S. northeast, Including CGM's Eagle Point Refinery in Westville, New Jersey.

Supplies sold to ProGold L.L.C. for its corn milling facilities in North Dakota.

(4) Some of these supplies are used via displacement, at the company's Eagle Point refinery in Westville, N.J. 


\section{VOLUME AND PRICE REPORT}

\section{Long-Term Imports}

Volumes (MMct/d) \& Prices (\$MMBTU) of Natural Gas Imported During the Past 12 Months 10/01/99 - 09/30/00

Long-Term Importer

\begin{tabular}{|c|c|c|c|c|c|c|c|c|c|c|c|c|c|c|}
\hline $\begin{array}{l}\text { Auth. } \\
\text { Vols. }\end{array}$ & $\begin{array}{l}1999 \\
\text { Oct. }\end{array}$ & $\begin{array}{l}1999 \\
\text { Nov. }\end{array}$ & $\begin{array}{l}1999 \\
\text { Dec. }\end{array}$ & $\begin{array}{l}2000 \\
\text { Jan. }\end{array}$ & $\begin{array}{l}2000 \\
\text { Feb. }\end{array}$ & $\begin{array}{l}2000 \\
\text { Mar. }\end{array}$ & $\begin{array}{l}2000 \\
\text { Apr. }\end{array}$ & $\begin{array}{l}2000 \\
\text { May. }\end{array}$ & $\begin{array}{l}2000 \\
\text { Jun. }\end{array}$ & $\begin{array}{l}2000 \\
\text { Jul. }\end{array}$ & $\begin{array}{l}2000 \\
\text { Aug. }\end{array}$ & $\begin{array}{l}2000 \\
\text { Sep. }\end{array}$ & $\begin{array}{l}3 Q 2000 \\
\text { TOTAL MCf }\end{array}$ & $\begin{array}{c}\text { YTD } \\
\text { TOTAL MCf }\end{array}$ \\
\hline 49.0 & $\begin{array}{l}49.5 \\
2.63 \\
2.63 \\
0.00 \\
0.00\end{array}$ & $\begin{array}{l}45.0 \\
3.25 \\
3.25 \\
0.00 \\
0.00\end{array}$ & $\begin{array}{r}0.0 \\
0.00 \\
0.00 \\
0.00 \\
0.00\end{array}$ & $\begin{array}{r}0.0 \\
0.00 \\
0.00 \\
0.00 \\
0.00\end{array}$ & $\begin{array}{r}0.0 \\
0.00 \\
0.00 \\
0.00 \\
0.00\end{array}$ & $\begin{array}{r}0.0 \\
0.00 \\
0.00 \\
0.00 \\
0.00\end{array}$ & $\begin{array}{r}0.0 \\
0.00 \\
0.00 \\
0.00 \\
0.00\end{array}$ & $\begin{array}{r}0.0 \\
0.00 \\
0.00 \\
0.00 \\
0.00\end{array}$ & $\begin{array}{r}0.0 \\
0.00 \\
0.00 \\
0.00 \\
0.00\end{array}$ & $\begin{array}{r}0.0 \\
0.00 \\
0.00 \\
0.00 \\
0.00\end{array}$ & $\begin{array}{r}0.0 \\
0.00 \\
0.00 \\
0.00 \\
0.00\end{array}$ & $\begin{array}{r}0.0 \\
0.00 \\
0.00 \\
0.00 \\
0.00\end{array}$ & 0 & 0 \\
\hline 30.5 & $\begin{array}{l}30.0 \\
2.51 \\
2.51 \\
0.00 \\
0.00\end{array}$ & $\begin{array}{l}30.9 \\
3.21 \\
3.21 \\
0.00 \\
0.00\end{array}$ & $\begin{array}{l}30.6 \\
2.14 \\
2.14 \\
0.00 \\
0.00\end{array}$ & $\begin{array}{l}29.9 \\
2.37 \\
2.37 \\
0.00 \\
0.00\end{array}$ & $\begin{array}{l}29.9 \\
2.63 \\
2.63 \\
0.00 \\
0.00\end{array}$ & $\begin{array}{r}3.1 \\
2.58 \\
2.58 \\
0.00 \\
0.00\end{array}$ & $\begin{array}{l}29.7 \\
2.98 \\
2.98 \\
0.00 \\
0.00\end{array}$ & $\begin{array}{l}29.8 \\
3.19 \\
3.19 \\
0.00 \\
0.00\end{array}$ & $\begin{array}{l}29.7 \\
4.49 \\
4.49 \\
0.00 \\
0.00\end{array}$ & $\begin{array}{l}29.7 \\
4.42 \\
4.42 \\
0.00 \\
0.00\end{array}$ & $\begin{array}{l}29.7 \\
3.92 \\
3.92 \\
0.00 \\
0.00\end{array}$ & $\begin{array}{l}29.7 \\
4.75 \\
4.75 \\
0.00 \\
0.00\end{array}$ & $2,731,392$ & $7,328,492$ \\
\hline 15.0 & $\begin{array}{r}2.0 \\
2.44 \\
2.44 \\
0.00 \\
0.00\end{array}$ & $\begin{array}{r}2.0 \\
2.96 \\
2.96 \\
0.00 \\
0.00\end{array}$ & $\begin{array}{r}2.0 \\
2.05 \\
2.05 \\
0.00 \\
0.00\end{array}$ & $\begin{array}{r}2.0 \\
2.27 \\
2.27 \\
0.00 \\
0.00\end{array}$ & $\begin{array}{r}2.0 \\
2.53 \\
2.53 \\
0.00 \\
0.00\end{array}$ & $\begin{array}{r}2.0 \\
2.52 \\
2.52 \\
0.00 \\
0.00\end{array}$ & $\begin{array}{r}2.0 \\
2.79 \\
2.79 \\
0.00 \\
0.00\end{array}$ & $\begin{array}{r}2.0 \\
3.00 \\
3.00 \\
0.00 \\
0.00\end{array}$ & $\begin{array}{r}2.0 \\
4.29 \\
4.29 \\
0.00 \\
0.00\end{array}$ & $\begin{array}{r}2.0 \\
4.26 \\
4.26 \\
0.00 \\
0.00\end{array}$ & $\begin{array}{r}2.0 \\
3.74 \\
3.74 \\
0.00 \\
0.00\end{array}$ & $\begin{array}{r}2.0 \\
4.53 \\
4.53 \\
0.00 \\
0.00\end{array}$ & 184,480 & 550,540 \\
\hline 9.0 & $\begin{array}{r}9.1 \\
2.64 \\
2.64 \\
0.00 \\
0.00\end{array}$ & $\begin{array}{r}9.1 \\
3.32 \\
3.32 \\
0.00 \\
0.00\end{array}$ & $\begin{array}{r}9.1 \\
2.23 \\
2.23 \\
0.00 \\
0.00\end{array}$ & $\begin{array}{r}9.1 \\
2.43 \\
2.43 \\
0.00 \\
0.00\end{array}$ & $\begin{array}{r}9.1 \\
2.70 \\
2.70 \\
0.00 \\
0.00\end{array}$ & $\begin{array}{r}9.1 \\
2.78 \\
2.78 \\
0.00 \\
0.00\end{array}$ & $\begin{array}{r}9.1 \\
3.08 \\
3.08 \\
0.00 \\
0.00\end{array}$ & $\begin{array}{r}9.1 \\
3.31 \\
3.31 \\
0.00 \\
0.00\end{array}$ & $\begin{array}{r}9.1 \\
4.61 \\
4.61 \\
0.00 \\
0.00\end{array}$ & $\begin{array}{r}9.1 \\
4.53 \\
4.53 \\
0.00 \\
0.00\end{array}$ & $\begin{array}{r}9.1 \\
4.03 \\
4.03 \\
0.00 \\
0.00\end{array}$ & $\begin{array}{r}9.1 \\
4.84 \\
4.84 \\
0.00 \\
0.00\end{array}$ & 832,692 & . $2,479,974$ \\
\hline 42.0 & $\begin{array}{l}40.0 \\
2.60 \\
2.60 \\
0.00 \\
0.00\end{array}$ & $\begin{array}{l}40.0 \\
3.16 \\
3.16 \\
0.00 \\
0.00\end{array}$ & $\begin{array}{l}40.0 \\
2.02 \\
2.02 \\
0.00 \\
0.00\end{array}$ & $\begin{array}{l}40.0 \\
2.23 \\
2.23 \\
0.00 \\
0.00\end{array}$ & $\begin{array}{l}41.4 \\
2.46 \\
2.46 \\
0.00 \\
0.00\end{array}$ & $\begin{array}{l}40.0 \\
2.02 \\
2.02 \\
0.00 \\
0.00\end{array}$ & $\begin{array}{l}40.0 \\
2.71 \\
2.71 \\
0.00 \\
0.00\end{array}$ & $\begin{array}{l}40.0 \\
2.88 \\
2.88 \\
0.00 \\
0.00\end{array}$ & $\begin{array}{l}40.0 \\
4.10 \\
4.10 \\
0.00 \\
0.00\end{array}$ & $\begin{array}{l}40.0 \\
4.17 \\
4.17 \\
0.00 \\
0.00\end{array}$ & $\begin{array}{l}40.0 \\
3.60 \\
3.60 \\
0.00 \\
0.00\end{array}$ & $\begin{array}{l}40.0 \\
4.52 \\
4.52 \\
0.00 \\
0.00\end{array}$ & $3,680,000$ & $11,000,000$ \\
\hline
\end{tabular}

ENGAGE ENERGY U.S., L.P.

(Various Suppliers)

AVG Daily Quantity

Total Price (St. Clair, Michigan)

Commodity Component

Demand Component

Reservation Fee Component

\section{ENGAGE ENERGY U.S., L.P.}

(Various Suppliers)

AVG Daily Quantity

Total Price (Noyes, Minnesota)

Commodity Component

Demand Component

Reservation Fee Component

ENRON NORTH AMERICA CORP. (1)

(ECTR - Canada)

AVG Daily Quantity

Total Price (Waddington, NY)

Commodity Component

Demand Component

Reservation Fee Component

ENRON NORTH AMERICA CORP. (2)

(ECTR - Canada)

Total Price (Niagara Falls, NY)

Commodity Component

Demand Component

Reservation Fee Component

ENRON NORTH AMERICA CORP. (3)

(ECTR - Canada)

AVG Daily Quantity

Total Price (Port of Mor

Demand Component

Reservation Fee Component

\section{.}


VOLUME AND PRICE REPORT

\section{Long-Term Imports}

Volumes (MMcf/d) \& Prices (\$/MMBTU) of Natural

Gas Imported During the Past 12 Months 10/01/99 - 09/30/00

Long-Term Importer

ENRON NORTH AMERICA CORP. (1)

(ECTR - Canada)

AVG Daily Quantity .

Total Price (Noyes, Minnesola)

Commodity Componen

Demand Component

Reservation Fee Component

ENRON NORTH AMERICA CORP.

(ECTR - Canada)

AVG Daily Quantity

Total Price (St. Clair. Michigan)

Commodity Component

Demand Component

Reservation Fee Component

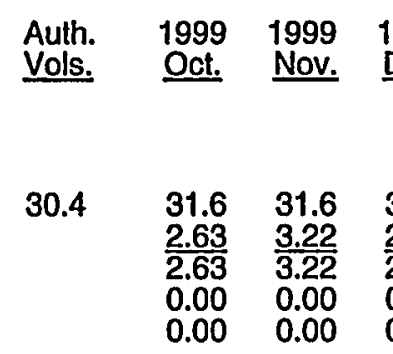

$19992000 \quad 2000 \quad 2000$

2000

2000

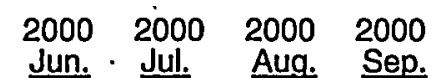

302000

IOTAL MCf

YTD

20.0

\begin{tabular}{rr}
0.0 & 20.3 \\
0.00 & 3.25 \\
\hline 0.00 & 3.25 \\
0.00 & 0.00 \\
0.00 & 0.00
\end{tabular}

31.6
$\frac{2.23}{2.23}$
0.00

31.7
2.35
2.35
0.00
0.00

31.5

31.3

$31.3 \quad 31.3$

26.3

$\frac{2.62}{2.62}$

$\frac{2.58}{2.58}$

$\frac{3.00}{3.00}$

$\frac{3.00}{3.00} \quad \overline{3.23}$

$\begin{array}{ll}0.00 & 0.00\end{array}$

0.00
0.00

$0.00 \quad 0.00$

$\frac{4.53}{4.53}$

0.00

26.3
$\frac{4.45}{4.45}$
0.00

26.4

$\frac{3.95}{3.95}$

3.95

26.3
4.76

4.76

0.00

$2,423,166$

$0.00 \quad 0.00$

ESSEX COUNTY GAS CO.

(Alberta NE Gas (TCGS Ltd. 1))

AVG Daily Quantity

Total Price (Waddington, NY)

Commodity Componen

Demand Component

Reservation Fee Component

GRANITE STATE GAS TRANSMISSION, INC. (2)

(Direct Energy Marketing Ltd.)

20.3
2.24
2.24
0.00
0.00

\begin{tabular}{l}
20.3 \\
2.35 \\
\hline
\end{tabular}

20.3

20.3

20.3

20.3

20.3

2.35

0.00
0.00

0.00

0.00

2.70

0.00
0.00

3.00

0.00
0.00

3.23

0.00
0.00

3.34

3.34
0.00
0.00

20.3
4.45

$\frac{4.45}{4.45}$

4.45

0.00

\begin{tabular}{l}
20.3 \\
2.35 \\
\hline 2.35
\end{tabular}

$\frac{2.35}{2.35}$

0.00
0.00

20.3

4.76

4.76
0.00

2.0

$\begin{array}{rrrr}2.0 & 1.3 & 2.0 & \\ 2.80 & \frac{3.22}{2.02} & \frac{2.36}{1.57} & \frac{2.5}{1.6} \\ 2.01 & 2.43 & 0.79 & 0.8 \\ 0.79 & 0.79 & 0.79 & \\ 0.00 & 0.00 & 0.00 & 0.00\end{array}$

\begin{tabular}{rr}
2.0 & 1.9 \\
2.52 & 2.77 \\
\hline 1.66 & 1.91 \\
0.86 & 0.86 \\
0.00 & 0.00
\end{tabular}

2.0
2.75
1.89
0.86
0.00

2.0
$\frac{3.11}{2.25}$
0.86
0.00

2.0
$\frac{3.30}{2.46}$
0.84
0.00

2.0
4.42
3.57

1.7
4.72
3.87

$\frac{4.72}{3.87}$

2.0
3.93
3.08

$\frac{3.93}{3.08}$

2.0
4.83
3.98

$\begin{array}{llll}0.85 & 0.85 & 0.85 & 0.85 \\ 0.00 & 0.00 & 0.00 & 0.00\end{array}$

\begin{tabular}{l}
35.0 \\
3.23 \\
\hline 2.60 \\
0.63 \\
0.00
\end{tabular}

\begin{tabular}{l}
35.0 \\
3.29 \\
\hline 3.02 \\
0.27 \\
0.00
\end{tabular}
AVG Daily Quantity

Total Price (Pittsburg, NH)

Demand Component

Reservation Fee Component

6.0

6.0
2.49
1.53
0.96
0.00

6.0
2.52
1.53
0.99
0.00

GRANITE STATE GAS TRANSMISSION, INC.

(Shell Canada Limited)

AVG Dally Quantity
Total Price (Waddington, NY)

Commodity Component

Demand Component

Reservation Fee Component

34.8

\begin{tabular}{lll}
35.0 & 35.0 & 35.0 \\
2.79 & $\frac{3.13}{2.26}$ & $\frac{2.65}{1.77}$ \\
\hline 1.91 & 2.26 \\
0.88 & 0.87 & 0.88 \\
0.00 & 0.00 & 0.00
\end{tabular}

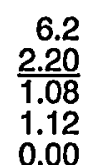

\begin{tabular}{r}
6.0 \\
2.13 \\
\hline 1.08 \\
1.05 \\
0.00
\end{tabular}

6.0
2.19
1.11
1.08
0.00

6.0
2.13
1.11
1.02
0.00

6.0
2.18
1.11
1.07
0.00

6.0
4.56
4.06
0.50

6.0
4.03
3.53

$0.50 \quad 0.50$

$0.95 \quad 1.05$

0.00

35.0
2.96
2.32
0.64
0.00

\begin{tabular}{l}
35.0 \\
3.28 \\
\hline 2.64 \\
0.64
\end{tabular}

\begin{tabular}{l}
35.0 \\
3.06 \\
\hline 2.79 \\
0.27 \\
0.00
\end{tabular}

\begin{tabular}{l}
35.0 \\
4.62 \\
\hline 4.35 \\
0.27 \\
0.00
\end{tabular}

\begin{tabular}{l}
35.0 \\
4.48 \\
\hline 4.21 \\
0.27 \\
0.00
\end{tabular}

35.0
3.95
3.68
0.27

35.0
4.75

$\frac{4.75}{4.48}$

4.48

0.27

6.0
.84
.34
.50
.00

552,202

$1,650,604$

(1) Primarily for markets in Michlgan.
(2) of $4 / 1 / 99$, this authorization began importing volumes at Pittsburg, NH. 
VOLUME AND PRICE REPORT

\section{Long-Term Imports}

Volumes (MMcf/d) \& Prices (\$/MMBTU) of Natural Gas Imported During the Past 12 Months 10/01/99 - 09/30/00

\section{Long-Term Importer}

Auth
Vols.

HERMISTON GENERATING COMPANY, L.P.

(Home Oil Company Limited)

Total Price (Eastport, Idaho)

Commodity Component

Demand Component

Reservation Fee Component

HERMISTON GENERATING COMPANY, L.P.

(TransCanada Gas Services L.td.)

AVG Daily Quantity

Total Price (Eastport, Idaho)

Commodity Component

Demand Component

Reservation Fee Component

HESS ENERGY INC. (1)

(Talisman Energy ino.)

AVG Daily Quantity
Total Price (Sumas, Washington)

Cotal Price (Sumas, Wastity Component

Demand Component

Reservation Fee Component

HESS ENERGY INC. (2)

(Talisman Energy Inc.)

AVG Daily Quantity

Total Price (Sumas, Washington)

Commodity Component

Demand Component

Reservation Fee Component

HUSKY GAS MARKETING INC. (3)

(Husky Oil Operations Ltd.)

AVG Daily Quantity

Total Price (Eastport, Idaho)

Commodity Component

Demand Component

Reservation Fee Component

15.0

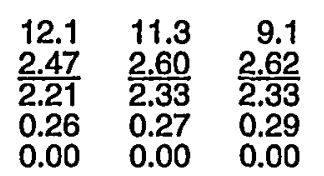

12.1
2.62
2.34
0.28
0.00

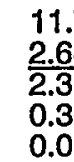

\begin{tabular}{lr}
11.7 & 9.1 \\
2.64 & 2.71 \\
\hline 2.33 & 2.33 \\
0.31 & 0.38 \\
0.00 & 0.00
\end{tabular}

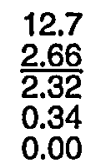

29.7

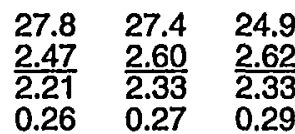

\begin{tabular}{l}
28.9 \\
2.62 \\
\hline 2.34 \\
0.28
\end{tabular}

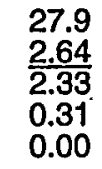

21.5
2.71
2.33

0.31
0.00

0.00

2.7

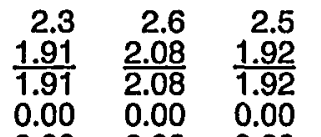

2.2
1.99
1.99
0.00

3.1
2.18
2.18
0.00

0.00
0.00

2.5
$\frac{2.12}{2.12}$
0.00
0.00

$\begin{array}{r}2.6 \\ 2.10 \\ \hline 2.10\end{array}$

$\frac{2.10}{2.10}$

0.00

0.00

2.8

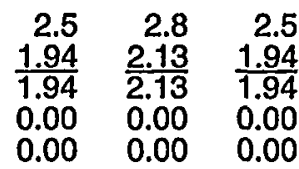

\begin{tabular}{r}
3.0 \\
2.20 \\
\hline 2.20 \\
0.00 \\
0.00
\end{tabular}

\begin{tabular}{r}
2.4 \\
1.99 \\
\hline 1.99 \\
0.00 \\
0.00
\end{tabular}

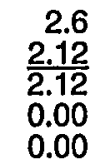

2.6
2.09

$\frac{2.09}{2.09}$

0.00
0.00

$\frac{2.01}{2.01}$

2.01
0.00

0.00

2.0
2.08
2.08
0.00

2.08
0.00

0.00
0.00

21.9

\begin{tabular}{lll}
18.7 & 15.5 & 21.8 \\
2.19 & 4.00 & $\frac{3.09}{3.09}$ \\
\hline 2.19 & 4.00 & 0.00 \\
0.00 & 0.00 & 0.00 \\
0.00 & 0.00 & 0.00
\end{tabular}

\begin{tabular}{l}
14.4 \\
2.83 \\
\hline 2.83 \\
0.00 \\
0.00
\end{tabular}

\begin{tabular}{l}
13.4 \\
3.39 \\
\hline 3.39 \\
0.00
\end{tabular}

\begin{tabular}{l}
11.1 \\
4.20 \\
\hline 4.20 \\
0.00 \\
0.00
\end{tabular}

\begin{tabular}{l}
21.6 \\
2.24 \\
\hline 2.24 \\
0.00 \\
0.00
\end{tabular}

\begin{tabular}{l}
20.9 \\
2.36 \\
\hline 2.36 \\
0.00
\end{tabular}
AVG Daily Quantily

$\begin{array}{llll}0.00 & 0.00 & 0.00 & 0.00\end{array}$

2.4
2.03
2.03
0.00

2.4
2.35
2.35
0.00

2.7
2.23
2.23

0.00

0.00

0.00

0.00

$\begin{array}{r}2.2 \\ 2.13 \\ \hline\end{array}$

2.13

0.00
0.00

$\begin{array}{r}2.9 \\ 2.14 \\ \hline\end{array}$

$\frac{2.14}{2.14}$

2.3

2.2
2.02

0.00
0.00

0.00
0.00

$\frac{2.02}{2.02}$

2.

$\frac{2.93}{2.93}$

2.93
0.00

0.00

(1) Hess Energy Inc. was formerly known as Statoil Energy Services, Inc. Supply used to fuel a 10 Megawatt gas-fired cogeneration facility near Glenns Ferry, Idaho.

(2) Hess Energy Inc. was formerly known as Statoil Energy Services, Inc. Supply used to fuel a 10 Megawatt gas-fired cogeneration facility near Rupert, Idaho.
(3) These volumes are delivered to San Diego Gas \& Electric Company.
302000

TOTAL MC

TOTAL

AL MCf

$1,267,743 \quad 3,438,989$

$2,510,135$

$7,214,340$

225,556

671,911

220,381

696,613

$1,893,497$

$4,984,268$ 


\section{Long-Term Importer}

IGI RESOURCES, INC

(Mobil Oil Canada)

AVG Daily Quantity
Total Price (Sumas, Washington)

Commodity Component

Demand Component

Reservation Fee Component

LOCKPORT ENERGY ASSOCIATES, L.P.

\section{(ProGas Limited)}

AVG Daily Quantity

Total Price (Niagara Falls, NY)

Commodity Componen

Demand Component

Reservation $\mathrm{Fee}$ Component

MIDLAND COGENERATION VENTURE1

(Anadarko Trading Company)

AVG Daily Quantity

Total Price (Noyes, Minnesota)

Demand Component

Reservation Fee Component

MIDLAND COGENERATION VENTURE2

\section{(Coral Energy)}

AVG Daily Quantity

Total Price (Noyes, Minnesota)

Commodity Componen

Demand Component

Reservation Fee Component

\section{MIDLAND COGENERATION VENTURE 3}

\section{(Husky Oil)}

AVG Daily Quantity

Total Price (Noyes, Minnesota)

Commodity Componen

Demand Component

Reservation Fee Component

\section{VOLUME AND PRICE REPORT}

\section{Long-Term Imports}

Volumes (MMcf/d) \& Prices (\$/MMBTU) of Natural

Gas Imported During the Past 12 Months 10/01/99 - 09/30/00

$\begin{array}{lllllllllll}\text { Auth. } & 1999 & 1999 & 1999 & 2000 & 2000 & 2000 & 2000 & 2000 & 2000 & 2000 \\ \text { Vols. } & \text { Oct } & & & & \end{array}$

Oct. Nov. Dec. Jan. Feb. Mar. Apr. May. Jun. Jul. Aug 2000

10.0

\begin{tabular}{rrrrrrrrrrrr}
0.0 & 0.0 & 0.0 & 0.0 & 0.0 & 0.0 & 0.0 & 0.0 & 0.0 & 0.0 & 0.0 & 0.0 \\
$\underline{0.00}$ & $\underline{0.00}$ & $\underline{0.00}$ & $\underline{0.00}$ & $\underline{0.00}$ & $\underline{0.00}$ & $\underline{0.00}$ & $\underline{0.00}$ & $\underline{0.00}$ & $\frac{0.00}{0.00}$ & $\underline{0.00}$ & $\underline{0.00}$ \\
\hline 0.00 & 0.00 & 0.00 & 0.00 & 0.00 & $\underline{0.00}$ & 0.00 & 0.00 & $\overline{0.00}$ & $\overline{0.00}$ & 0.00 & 0.00 \\
0.00 & 0.00 & 0.00 & 0.00 & 0.00 & 0.00 & 0.00 & 0.00 & 0.00 & 0.00 & 0.00 & 0.00 \\
0.00 & 0.00 & 0.00 & 0.00 & 0.00 & 0.00 & 0.00 & 0.00 & 0.00 & 0.00 & 0.00 & 0.00
\end{tabular}

12.0

\begin{tabular}{rr}
4.9 & 4.9 \\
5.28 & 5.60 \\
\hline 2.79 & 2.96 \\
2.13 & 2.24
\end{tabular}

$11.8 \quad 8.8$

$\frac{4.01}{2.96}$

2.96

$\frac{4.48}{2.95}$

$\begin{array}{ll}12.0 & 3.8\end{array}$

$\frac{4.15}{2.95}$

$\begin{array}{ll}0.36 & 0.40\end{array}$

0.160 .22

$1.03 \quad 3.02$

$\begin{array}{ll}0.17 & 0.50\end{array}$

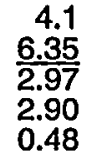

\begin{tabular}{rr}
4.1 & 2.6 \\
6.19 & 8.18 \\
\hline & 2.93
\end{tabular}

$\frac{6.19}{2.95}$

2.93

$\begin{array}{r}7.1 \\ 4.81 \\ \hline\end{array}$

$\frac{4.81}{2.94}$

2.94
1.60

$9.7 \quad 12.3$

$\frac{4.33}{2.95}$

$\frac{4.10}{2.96}$

0.49

.75

0.20

0.16

10.0

$\begin{array}{lll}10.0 & 10.0 & 10.0 \\ \frac{1.64}{1.19} & \frac{1.66}{1.19} & \frac{1.64}{1.18} \\ 0.45 & 0.47 & 0.46 \\ 0.00 & 0.00 & 0.00\end{array}$

$\begin{array}{ll}10.0 & 10.0 \\ 1.63 & 1 \\ 1.12 & 1.12 \\ 0.00 & 0.54 \\ 0.00 & 0.00\end{array}$

\begin{tabular}{l}
10.0 \\
1.66 \\
\hline 1.12 \\
0.54 \\
0.00
\end{tabular}

\begin{tabular}{l}
10.0 \\
1.64 \\
\hline 1.13 \\
0.51 \\
0.00
\end{tabular}

10.0

10.0

$\frac{1.65}{1.13}$

$\frac{1.65}{1.15}$

10.0

$\frac{10.0}{1.66}$

10.0

0.00

0.00

0.00

$\frac{1.64}{1.14}$

10.0

$\frac{1.64}{1.14}$

10.0

$\frac{1.66}{1.14}$

$\begin{array}{lll}0.50 & 0.50 & 0.52 \\ 0.00 & 0.00 & 0.00\end{array}$

15.0

$\begin{array}{lll}15.0 & 15.0 & 15.0 \\ \frac{1.57}{1.15} & \frac{1.58}{1.15} & \frac{1.57}{1.15} \\ 0.42 & 0.43 & 0.42\end{array}$

$\begin{array}{ll}15.0 & 15.0 \\ 154 & 157\end{array}$

15.0
$\frac{1.57}{1.07}$

$0.47 \quad 0.50$

0.00

\begin{tabular}{l}
15.0 \\
2.00 \\
\hline 1.54 \\
0.46
\end{tabular}

$\begin{array}{ll}15.0 & 15.0 \\ 1.56 & 15\end{array}$

$\frac{1.56}{1.07} \cdot \frac{1.54}{1.08}$

$0.49 \quad 0.46$

$0.00 \quad 0.00$

\begin{tabular}{l}
15.0 \\
1.56 \\
\hline 1.08 \\
0.48 \\
0.00
\end{tabular}

15.

$\frac{1.55}{1.09}$

15.0

$\begin{array}{ll}0 & 15.0\end{array}$

15.0
1.51
1.09

$\frac{1.51}{1.09}$

$0.46 \quad 0.42$

$\begin{array}{lll}0.00 & 0.00 & 0.00\end{array}$

15.0

\begin{tabular}{lll}
15.0 & 15.0 & 15.0 \\
1.90 & 1.90 & 1.90 \\
\hline 1.49 & $\frac{1.47}{1.49}$ \\
0.41 & 0.43 & 0.41 \\
0.00 & 0.00 & 0.00
\end{tabular}

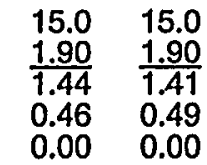

15.0
1.90
1.44
0.46
0.00

$\begin{array}{ll}15.0 & 15.0 \\ 1.90 & 1.90 \\ 1.42 & 1.45 \\ 0.48 & 0.45 \\ 0.00 & 0.00\end{array}$

$\begin{array}{ll}15.0 & 15.0\end{array}$

$\frac{1.90}{1.44}$

0.46

$\begin{array}{r}15.0 \\ 1.90 \\ \hline 1.44\end{array}$

$\frac{1.90}{1.44}$

1.49

0.00
0.47
0.00 $\begin{array}{lll}0.00 & 0.00 & 0.00\end{array}$
890,519

$1,957,967$

920,000

$2,740,000$

$1,380,000$

$4,110,000$

0

TOTAL

.0
.90
.43
.47
.00

.90
.43
.47
.00


VOLUME AND PRICE REPORT

\section{Long-Term Imports}

Volumes (MMcf/d) \& Prices (\$/MMBTU) of Natural

Gas Imported During the Past 12 Months 10/01/99 - 09/30/00

Long-Term Importer

MIDLAND COGENERATION VENTURE4

(TransCanada Pipelines Limited)

AVG Daily Quantity

Total Price (Noyes, Minnesota)

Commodity Component

Demand Component

Reservation Fee Component

MIDLAND COGENERATION VENTURE5 (Burlington Resources Canada Energy Ltd.)

AVG Daily Quantity

Total Price (Noyes, Minnesota)

Commodity Component

Demand Component

Reservation Fee Component

MIDLAND COGENERATION VENTURE6

(Anadarko Trading Company)

AVG Daily Quantity

Total Price (Noyes, Minnesota)

Commodity Component

Demand Component

Reservation Fee Component

MINNEGASCO, INC.

(TransCanada Pipelines Limited)

AVG Daily Quantity

Total Price (Noyes, Minnesola)

Commodity Component

Demand Component

Reservation Fee Component

N.Y. STATE ELECTRIC \& GAS CO.

(Alberta NE Gas (AEC Oil \& Gas Co.))

AVG Dally Quantity

Total Price (Waddington, NY)

Commodity Component

Demand Component

Reservation Fee Component
Auth.

Vols

\begin{tabular}{ll}
15.0 & 15.0 \\
1.56 & 1.57 \\
\hline 1.14 & 1.14 \\
0.42 & 0.43 \\
0.00 & 0.00
\end{tabular}

15.0
1.55
1.14
0.41
0.00

15.0
1.55

$\frac{1.55}{1.08}$

$\begin{array}{llll}0.00 & 0.00 & 0.00 & 0.47\end{array}$

$\frac{1.57}{1.08}$

1.08
0.49

0.49
0.00

$\frac{1.55}{1.09}$

1.09
0.46
0.00

1.56
1.08
0.48
0.00

$\frac{1.55}{1.10}$

1.10
0.45

0.45
0.00

$\frac{1.56}{1.09}$

1.09
0.47
0.00

$\frac{1.55}{1.09}$

0.46

1.56

1.10

$\frac{1.57}{1.10}$

1.10
0.47

\begin{tabular}{l}
15.0 \\
2.56 \\
\hline 2.10 \\
0.46 \\
0.00
\end{tabular}

15.0
2.58
2.10
0.48
0.00
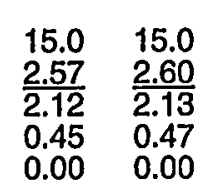

10.0

\begin{tabular}{rrr}
0.0 & 10.0 & 10 \\
0.00 & 2.99 & 2.98 \\
\hline 0.00 & 2.59 & 2.59 \\
0.00 & 0.40 & 0.39 \\
0.00 & 0.00 & 0.00
\end{tabular}

50.0

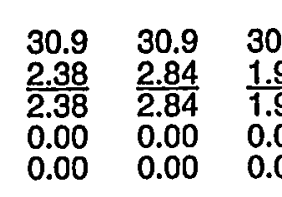
\begin{tabular}{l}
10.0 \\
3.00 \\
\hline
\end{tabular}

$\frac{3.00}{2.58}$

\begin{tabular}{l}
10.0 \\
3.04 \\
\hline 2.59
\end{tabular}

3.04
2.59
0.45

\begin{tabular}{l}
10.0 \\
3.01 \\
\hline 2.60 \\
0.41 \\
0.00
\end{tabular}

\begin{tabular}{l}
10.0 \\
3.04 \\
\hline 2.62 \\
0.42 \\
0.00
\end{tabular}

\begin{tabular}{l}
10.0 \\
3.03 \\
\hline 2.63 \\
0.40 \\
0.00
\end{tabular}

$\begin{array}{lll}0.00 & 0.00 \cdot 0.00\end{array}$

0.40
0.00

\begin{tabular}{l}
10.3 \\
3.06 \\
\hline 2.64 \\
0.42 \\
0.00
\end{tabular}

0.42
0.00

\begin{tabular}{l}
15.0 \\
2.60 \\
\hline 2.14 \\
0.46 \\
0.00
\end{tabular}

\begin{tabular}{l}
15.0 \\
2.61 \\
\hline 2.15 \\
0.46 \\
0.00
\end{tabular}

\begin{tabular}{l}
15.0 \\
2.63 \\
\hline 2.16 \\
0.47 \\
0.00
\end{tabular}

$1,380,000$

$4,110,000$

$1,380,000 \quad 4,110,000$

$1,380,000$

(2)

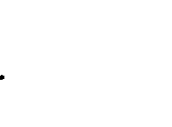

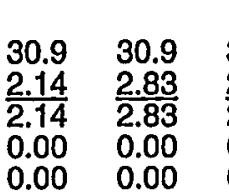
30.9
2.36
$\frac{2.36}{2.36}$

\begin{tabular}{l}
30.9 \\
2.73 \\
\hline 2.73 \\
0.00
\end{tabular}

\begin{tabular}{r}
30.9 \\
$\cdot 2.91$ \\
\hline 2.91 \\
0.00 \\
0.00
\end{tabular}

\begin{tabular}{l}
30.9 \\
4.04 \\
\hline 4.04
\end{tabular}

$\frac{4.04}{4.04}$

$\begin{array}{ll}0.00 & 0.00\end{array}$

$\begin{array}{ll}0.00 & 0.00\end{array}$

\begin{tabular}{l}
10.0 \\
3.05 \\
\hline 2.65 \\
0.40 \\
0.00
\end{tabular}

\begin{tabular}{l}
10.0 \\
3.06 \\
\hline 2.66 \\
0.40
\end{tabular}

0.400

\begin{tabular}{l}
10.3 \\
3.09 \\
\hline 2.67 \\
0.42 \\
0.00
\end{tabular}

1.3

\begin{tabular}{rrr}
1.3 & 0.6 & 1.3 \\
2.94 & $\frac{3.34}{2.46}$ & $\frac{2.67}{1.79}$ \\
\hline 2.06 & 2.46 & 0.88 \\
0.88 & 0.88 & 0.88 \\
0.00 & 0.00 & 0.00
\end{tabular}

0.00

\begin{tabular}{lll}
30.9 & 30.9 & 30.9 \\
4.34 & $\frac{3.55}{3.55}$ & $\frac{4.44}{4.44}$ \\
\hline 4.34 & 3.55 & 0.00 \\
0.00 & 0.00 & 0.00 \\
0.00 & 0.00 & 0.00
\end{tabular}

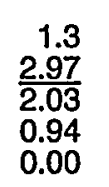

\begin{tabular}{r}
1.3 \\
3.32 \\
\hline 2.38 \\
0.94 \\
0.00
\end{tabular}

0.0
0.00
0.00
0.00
0.00

0.0
0.00
0.00
0.00
0.00

\begin{tabular}{r}
1.3 \\
3.57 \\
\hline 2.65 \\
0.92 \\
0.00
\end{tabular}

1.3
4.72
3.79
0.93
0.00

1.1
4.71
3.78
0.93
0.00

\begin{tabular}{rr}
1.3 & 1.3 \\
4.33 & 4.92 \\
\hline 3.41 & 4.00 \\
0.92 & 0.92 \\
0.00 & 0.00
\end{tabular}

115,362

275,808 
VOLUME AND PRICE REPORT

\section{Long-Term Imports}

Volumes (MMcf/d) \& Prices (\$/MMBTU) of Natural

Gas Imported During the Past 12 Months 10/01/99 - 09/30/00

Long-Term Importer

N.Y. STATE ELECTRIC \& GAS CO.

(Alberta NE Gas (TCGS Ltd. 2))

AVG Daily Quantity

Total Price (Waddington, NY)

Commodity Component

Demand Component

Reservation Fee Component

N.Y. STATE ELECTRIC \& GAS CO

(Alberta Northeast Gas (ProGas))

AVG Daily Quantity

Total Price (Waddington, NY)

Commodity Componen

Demand Component

Reservation Fee Component

N.Y. STATE ELECTRIC \& GAS CO

(Alberta Northeast Gas (Producers Marketing))

AVG Daily Quantity

Total Price (Waddington. NY)

Commodity Componen

Demand Component

Reservation Fee Component

N.Y. STATE ELECTRIC \& GAS CO.

(Crestar Energy)

Total Price (Grand Island, NY)

Commodity Component

Demand Component

Reservation Fee Component

N.Y. STATE ELECTRIC \& GAS CO. (1)

(Progas Limited)

Total Price (Niagara Falls, NY)

Commodity Componen

Demand Component

Reservation Fee Component
AVG Daily Quantity
Auth

\begin{tabular}{ccccc}
1999 & 1999 & 1999 & 2000 & 2000 \\
Oct. & Nov. & Dec. & Jan. & Feb. \\
\hline
\end{tabular}

20002000

Vols.

6.8

\begin{tabular}{rr}
6.8 & 4.3 \\
2.80 & 3.22 \\
\hline 2.01 & 2.43 \\
0.79 & 0.79 \\
0.00 & 0.00
\end{tabular}

6.8
2.36
1.57
0.79
0.00

$\begin{array}{r}6.8 \\ 2.52 \\ \hline\end{array}$

$\frac{2.52}{1.66}$

$0.00 \quad 0.00$

5.9

\begin{tabular}{rr}
5.9 & 2.1 \\
2.99 & 3.38 \\
\hline 2.07 & 2.48 \\
0.92 & 0.90
\end{tabular}

$\begin{array}{ll}0.92 & 0.90 \\ 0.00 & 0.00\end{array}$

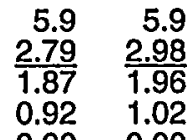

$0.92 \quad 1.02$

$0.00 \quad 0.00$

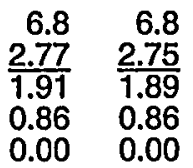

\begin{tabular}{r}
6.8 \\
3.11 \\
\hline 2.25 \\
0.86 \\
0.00
\end{tabular}

\begin{tabular}{r}
6.8 \\
3.30 \\
\hline 2.46 \\
0.84 \\
0.00
\end{tabular}

\begin{tabular}{r}
6.8 \\
4.42 \\
\hline 3.57 \\
0.85 \\
0.00
\end{tabular}

5.7
$\frac{4.72}{3.87}$

3.87
0.85

0.85
0.00

$\frac{3.93}{3.08}$

0.85
0.00

4.83

3.98

0.85

3.0

\begin{tabular}{rrr}
3.0 & 2.1 & \\
2.99 & $\frac{3.37}{2.44}$ & $\frac{2}{1}$ \\
\hline 2.10 & 2.80 \\
0.89 & 0.93 & 0.8 \\
0.00 & 0.00 & 0.00
\end{tabular}

10.0

\begin{tabular}{rr}
9.6 & 6.3 \\
3.68 & 4.49 \\
\hline 2.78 & 3.08 \\
0.90 & 1.41 \\
0.00 & 0.00
\end{tabular}

\begin{tabular}{r}
6.3 \\
4.49 \\
\hline 3.08 \\
1.41 \\
0.00
\end{tabular}

7.9
3.71
2.61
1.10
0.00

\begin{tabular}{r}
9.9 \\
3.55 \\
\hline 2.60 \\
0.95 \\
0.00
\end{tabular}

$\begin{array}{r}7.9 \\ 4.03 \\ \hline\end{array}$

$\frac{4.03}{2.75}$

1.28
0.00

$\begin{array}{r}8.97 \\ \hline 2.84\end{array}$

$\frac{3.97}{2.84}$

1.13
0.00

\begin{tabular}{l}
10.0 \\
4.07 \\
\hline 3.10
\end{tabular}

3.10

0.97
0.00

8.4
.4 .41
3.31

3.31

1.10
0.00

\begin{tabular}{l}
10.0 \\
5.02 \\
\hline 4.05
\end{tabular}

0.97
0.00

$\frac{8.18}{3.36}$

4.82

0.96

$0.00 \quad 0.00$

9.0

$\begin{array}{rrr}8.1 & 7.4 & 7.9 \\ \frac{3.17}{2.23} & \frac{3.27}{2.19} & \frac{3.18}{2.20} \\ 0.94 & 1.08 & 0.98 \\ 0.00 & 0.00 & 0.00\end{array}$

\begin{tabular}{r}
8.0 \\
3.36 \\
\hline 2.31 \\
1.05 \\
0.00
\end{tabular}

\begin{tabular}{r}
8.0 \\
3.85 \\
\hline 2.73 \\
1.12 \\
0.00
\end{tabular}

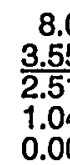

\begin{tabular}{r}
8.0 \\
3.55 \\
\hline 2.51 \\
1.04 \\
0.00
\end{tabular}

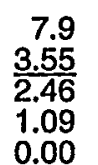

8.0
$\frac{3.56}{2.53}$
1.03
0.00

8.0
$\frac{3.91}{2.84}$
1.07
0.00

8.0
4.02
2.98
1.04

\begin{tabular}{r}
8.0 \\
3.48 \\
\hline 2.45 \\
1.03
\end{tabular}

8.0

$\frac{3.88}{2.82}$

2.82

1.06
$587,250 \quad 1,815,750$

516,780

$1,597,860$

259,608

802,696

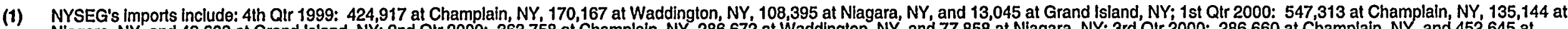

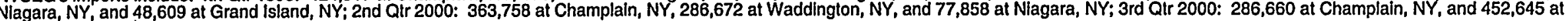

Waddington, NY. 


\section{VOLUME AND PRICE REPORT}

\section{Long-Term Imports}

Volumes (MMcf/d) \& Prices (\$/MMBTU) of Natural

Gas Imported During the Past 12 Months 10/01/99 - 09/30/00

Long-Term Importer

NATIONAL FUEL GAS DISTRIBUTION CORP.

(Alberta NE Gas (TCGS Lid.))

AVG Daily Quantity

Total Price (Niagara Falls, NY)

Comand Component

Reservation Fee Component

\section{NATIONAL STEEL CORPORATION}

(Direct Energy Marketing, Ltd.)

AVG Dally Quantity

Total Price (Detroit, Michigan)

Commodity Componen

Demand Component

Reservation Fee Component

NEW JERSEY NATURAL GAS COMPANY

(Alberta NE Gas (AEC Oil \& Gas Company))

AVG Daily Quantity

Total Price (Waddington, NY)

Commodity Componen

Demand Component

Reservation Fee Component

NEW JERSEY NATURAL GAS COMPANY

(Alberta NE Gas (TCGS Ltd. 1))

AVG Daily Quantity

Total Price (Waddington, NY)

Commodity Componen

Demand Component

Reservation Fee Component

NEW JERSEY NATURAL GAS COMPANY

(Alberta NE Gas (TCGS Ltd. 2))

AVG Daily Quantity

Total Price (Waddington, NY)

Commodity Component

Demand Component

Reservation Fee Component
10.0
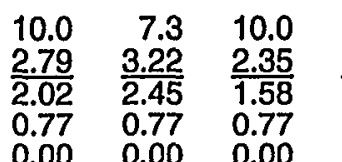

\begin{tabular}{l}
10.0 \\
2.52 \\
\hline 1.68 \\
0.84 \\
0.00
\end{tabular}

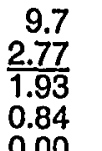

\begin{tabular}{ll}
10.0 & 10.0 \\
2.75 & $\frac{3.11}{2.27}$ \\
\hline 1.91 & 0.84 \\
0.00 & 0.00
\end{tabular}

\begin{tabular}{ll}
10.0 & 10.0 \\
3.11 & $\frac{3.30}{2.48}$ \\
\hline 2.27 & 0.82 \\
0.84 & 0.82 \\
0.00 & 0.00
\end{tabular}

\begin{tabular}{ll}
10.0 & 10.0 \\
3.30 & 4.42 \\
\hline 2.48 & 3.59 \\
0.82 & 0.83 \\
\hline .00 & 0.00
\end{tabular}

\begin{tabular}{ll}
0 & 10.0 \\
\hline 2 & 4.72 \\
\hline 3.89 \\
\hline 3 & 0.83 \\
\hline 0 & 0.00
\end{tabular}

10.0
3.93

$\frac{3.93}{3.10}$

$\begin{array}{ll}3.10 & 4.00 \\ 0.83 & 0.83\end{array}$

$0.00 \quad 0.00$

18.0

$\begin{array}{llllllllllll}22.5 & 21.5 & 25.2 & 28.7 & 19.4 & 19.6 & 17.1 & 15.9 & 15.8 & 18.7 & 21.3 & 14.5 \\ \frac{2.43}{2.43} & \frac{2.47}{2.47} & \frac{2.62}{2.62} & \frac{2.94}{2.94} & \frac{2.97}{2.97} & \frac{2.76}{2.76} & \frac{2.98}{2.98} & \frac{3.02}{3.02} & \frac{3.12}{3.12} & \frac{3.31}{3.31} & \frac{3.81}{3.81} & \frac{3.68}{3.68} \\ 0.00 & 0.00 & 0.00 & 0.00 & 0.00 & 0.00 & 0.00 & 0.00 & 0.00 & 0.00 & 0.00 & 0.00 \\ 0.00 & 0.00 & 0.00 & 0.00 & 0.00 & 0.00 & 0.00 & 0.00 & 0.00 & 0.00 & 0.00 & 0.00\end{array}$

1.9

$\begin{array}{rrrr}1.9 & 1.2 & 1.9 & 1.9 \\ \frac{2.94}{2.06} & \frac{3.34}{2.46} & \frac{2.67}{1.79} & \frac{2.97}{2.03} \\ 0.88 & 0.88 & 0.88 & 0.94 \\ 0.00 & 0.00 & 0.00 & 0.00\end{array}$

1.9
$\frac{3.32}{2.38}$
0.94
0.00

\begin{tabular}{r}
0.0 \\
0.00 \\
\hline 0.00 \\
0.00
\end{tabular}

$\begin{array}{r}0.0 \\ 0.00 \\ \hline 0.00\end{array}$

0.00

$0.00 \quad 0.00$

25.2

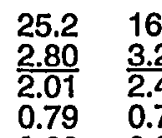

16.0
$\frac{3.22}{2.43}$
0.79

\begin{tabular}{l}
25.2 \\
2.36 \\
\hline 1.57 \\
0.79 \\
0.00
\end{tabular}

\begin{tabular}{l}
25.2 \\
2.52 \\
\hline 1.66 \\
0.86 \\
0.00
\end{tabular}

\begin{tabular}{ll}
25.2 & 25.2 \\
2.77 & 2.75 \\
\hline 1.91 & 1.89 \\
0.86 & 0.86 \\
0.00 & 0.00
\end{tabular}

$25.2 \quad 25.2$

$\frac{3.11}{2.25}$

0.00

0.00

5.3

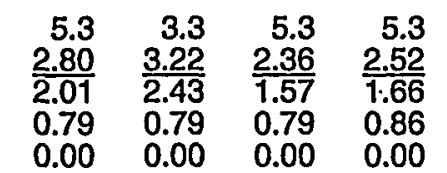

\begin{tabular}{rr}
5.3 & 5.3 \\
2.77 & 2.75 \\
\hline 1.91 & 1.89 \\
0.86 & 0.86 \\
0.00 & 0.00
\end{tabular}

\begin{tabular}{r}
5.3 \\
3.11 \\
\hline 2.25 \\
0.86 \\
0.00
\end{tabular}

\begin{tabular}{r}
1.9 \\
3.57 \\
\hline 2.65 \\
0.92 \\
0.00
\end{tabular}

1.9
4.72
3.79

$\begin{array}{r}1.9 \\ 4.71 \\ \hline 3.78\end{array}$

1.9
3.41

4.33
3.41
0.92

$\frac{4.92}{4.00}$

0.93
0.00

0.93
0.00

$0.00 \quad 0.00$

0.00
3Q 2000

TOTAL MG

920,000

$2,730,000$

$1,674,973$

$5,218,249$

177,086

412,552

$2,293,200$

$6,879,600$

472,750

$1,428,250$ 


\section{VOLUME AND PRICE REPORT}

\section{Long-Term Imports}

Volumes (MMcf/d) \& Prices (\$MMBTU) of Natural

Gas Imported During the Past 12 Months 10/01/99 - 09/30/00

Long-Term Importer

NEW JERSEY NATURAL GAS COMPANY

(Alberta Northeast Gas (ProGas))

AVG Daily Quantity

Total Price (Waddington, NY)

Commodity Componen

Demand Component

Reservation Fee Component

NEW JERSEY NATURAL GAS COMPANY

(Alberta Northeast Gas (Producers Mktg.))

AVG Daily Quantity

Total Price (Waddington, NY)

Commodity Component

Demand Component

Reservation Fee Component

NIAGARA MOHAWK POWER CORPORATION (TCGS Ltd.)

AVG Daily Quantity

Total Price (Waddington, NY)

Commodity Component

Demand Component

Reservation Fee Component

\section{NORTH JERSEY ENERGY ASSOCIATES}

(ProGas Limited)

AVG Daily Quantity

Total Price (Niagara Falls, NY)

Commodity Componen

Demand Component

Reservation Fee Component

\section{NORTHEAST ENERGY ASSOCIATES}

(ProGas Limited)

AVG Daily Quantity

Total Price (Niagara Falls, NY)

Commodity Componen

Demand Component

Reservation Fee Component
Vols. Oct. Nov. Dec. Jan. Feb. Mar. Apr. Mav. Jun. Jul. Aug. Sep.

4.6

\begin{tabular}{rrrr}
4.6 & 1.9 & 4.6 & 4.6 \\
2.99 & $\frac{3.38}{2.07}$ & 2.79 & 2.98 \\
\hline 0.92 & 0.90 & 0.97 & 1.96 \\
0.00 & 0.00 & 0.00 & 0.02
\end{tabular}

\begin{tabular}{r}
4.6 \\
3.42 \\
\hline 2.40 \\
1.02
\end{tabular}

$\begin{array}{r}4.6 \\ 3.36 \\ \hline\end{array}$

$\frac{3.36}{2.35}$

1.01

1.02
0.00

0.00

$\begin{array}{r}4.6 \\ 3.49 \\ \hline\end{array}$

2.47

1.02
0.00

$\begin{array}{r}4.6 \\ 3.61 \\ \hline\end{array}$

$\frac{3.61}{2.61}$

1.00

0.00

$\begin{array}{r}4.6 \\ 4.70 \\ \hline\end{array}$

$\frac{4.70}{3.69}$

1.01

1.01
0.00

$\begin{array}{r}4.5 \\ 4.76 \\ \hline 3.75\end{array}$

3.75
1.01

3.0

$\begin{array}{rrrr}3.0 & 1.9 & 3.0 & \\ \frac{2.99}{2.10} & \frac{3.37}{2.44} & \frac{2.79}{1.90} & 2.9 \\ 0.89 & 0.93 & 0.89 & 0.96 \\ 0.00 & 0.00 & 0.00 & 0.01\end{array}$

3.0
2.98
1.99
0.99
0.00

3.0

$\frac{3.42}{2.44}$

3.0

3.0
$\frac{3.37}{2.39}$

3.0

$\frac{3.49}{2.51}$

3.0

0.98

0.98

0.00

51.0

\begin{tabular}{lll}
51.0 & 51.6 & 51.6 \\
2.97 & 3.48 & 2.48 \\
\hline 2.12 & 2.59 & 1.63 \\
0.85 & 0.89 & 0.85 \\
0.00 & 0.00 & 0.00
\end{tabular}

51.0
2.73
1.79
0.94
0.00

\begin{tabular}{l}
51.0 \\
3.08 \\
\hline 2.08
\end{tabular}

51.0
3.00
2.07
0.93

0.00

$0.00 \quad 0.96$
0.00

51.0
$\frac{3.30}{2.34}$
0.96
0.00

\begin{tabular}{l}
51.0 \\
3.47 \\
\hline 2.56 \\
0.91 \\
0.00
\end{tabular}

\begin{tabular}{l}
51.0 \\
4.88 \\
\hline 3.98
\end{tabular}

$\frac{4.88}{3.93}$

3.0

$\frac{3.69}{2.72}$

2.9

$\frac{4.75}{3.78}$

$\frac{4.33}{3.36}$

$\begin{array}{llll}0.00 & 0.90 & 0.00 & 0.00\end{array}$

24.0

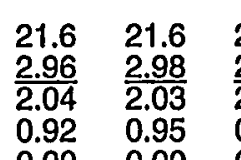

21.6
2.96
2.04
0.92
0.00

21.6
$\frac{3.32}{2.31}$
1.01
0.00

21.6

$\frac{3.39}{2.31}$

21.6

$\frac{3.31}{2.31}$

\begin{tabular}{l}
21.6 \\
3.35 \\
\hline
\end{tabular}

$\frac{3.35}{2.31}$

1.08
0.00

1.00
0.00

0.00

21.6
$\frac{3.32}{2.33}$
0.99

22.1
$\frac{3.33}{2.32}$
1.01
0.00

22.3

$\frac{3.19}{2.2}$

$0.00 \quad 0.00$

51.0
4.82
3.90

$\frac{4.82}{3.90}$

48.0

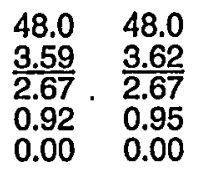

\begin{tabular}{l}
48.0 \\
3.59 \\
\hline 2.67 \\
0.92 \\
0.00
\end{tabular}

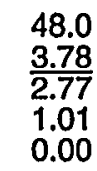

\begin{tabular}{l}
48.0 \\
3.85 \\
\hline 2.77 \\
1.08 \\
0.00
\end{tabular}

$\begin{array}{ll}48.0 & 46.4\end{array}$

$\frac{3.77}{2.77}$

2.77

1.00
0.00

$\begin{array}{r}46.4 \\ 3.84 \\ \hline 2.77\end{array}$

2.77
1.07

0.00
42.6

$\frac{3.90}{2.79}$

2.79
1.11

0.00
$49.1 \quad 49.4$

$\frac{3.76}{2.75} \quad \frac{3.72}{2.75}$

$1.01 \cdot 0.97$

$0.00 \quad 0.00$ $\begin{array}{r}4.63 \\ \hline 3.33\end{array}$

$\frac{4.33}{3.33}$

1.00

5.05

4.05

1.00
0.00

$\begin{array}{r}3.0 \\ 4.33 \\ \hline\end{array}$

3.0

$\frac{5.06}{4.09}$

4.09
0.97

51.0

51.0

$\frac{5.11}{4.16}$

4.16
0.95

3Q 2000

TOTAL MCf

420,420

$1,261,260$

271,544

814,632

$4,692,000$

$13,974,000$

$22.3 \quad 22.3$

$\frac{3.19}{2.22} \quad \frac{3.22}{222}$

$0.97 \quad 1.00$

$0.00 \quad 0.00$

$2,049,024$

$6,002,835$

$49.4 \quad 49.4$

$\frac{3.73}{2.76} \quad \frac{3.76}{2.76}$

$2.76 \quad 2.76$

$0.00 \quad 0.00$ 
VOLUME AND PRICE REPORT

\section{Long-Term Imports}

Volumes (MMcf/d) \& Prices (\$MMBTU) of Natural

Gas Imported During the Past 12 Months 10/01/99 - 09/30/00
Long-Term Importer

\section{NORTHERN MINNESOTA UTILITIES}

(TCGS Ltd.)

AVG Daily Quantity

Commodity Componen

Demand Component

Reservation Fee Component
Total Price (Noyes, Minnesota) $\begin{array}{lll}\text { Auth. } & 1999 & 1999 \\ \text { Vols. } & \text { Oct. Nov. }\end{array}$

10.0

$\begin{array}{lll}10.0 & 10.0 & 10.0 \\ \frac{2.33}{1.91} & \frac{2.76}{2.33} & \frac{1.89}{1.48} \\ 0.42 & 0.43 & 0.41 \\ 0.00 & 0.00 & 0.00\end{array}$

9992000

Dec.

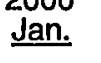

2000

2000

2000

2000

2000

\begin{tabular}{lll}
10.0 & 10.0 & 10.0 \\
2.06 & 2.30 & 2.28 \\
\hline 1.59 & $\frac{2.81}{1.82}$ \\
0.47 & 0.49 & 0.46 \\
0.00 & 0.00 & 0.00
\end{tabular}

10.0
2.65
2.17
0.48
0.00

\begin{tabular}{l}
10.0 \\
3.06 \\
\hline 2.61
\end{tabular}

$\frac{3.06}{2.61}$

0.45

0.00

10.0
4.20
3.73
0.47
0.00

10.0
4.17

$\frac{4.17}{3.71}$

0.46
0.00

$\frac{3.58}{3.12}$

3.46
0.00

$0.00 \quad 0.00$

NORTHERN MINNESOTA UTILITIES - EASTERN MARKET (1)

(TCGS Ltd.)

AVG Daily Quantity

Total Price (International Falls, MN)

29.8

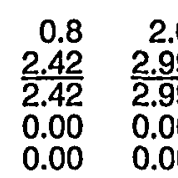

3.2
2.14
2.14
0.00
0.00

\begin{tabular}{r}
3.4 \\
2.32 \\
\hline 2.32 \\
0.00 \\
0.00
\end{tabular}

$\frac{2.56}{2.56}$

Demand Component

0.00

Reservation Fee Component

MARKET

(TCGS Ltd)

AVG Daily Quantity

Total Price (Warroad, Minnesota)

Commodity Componen

Demand Component

Reservation Fee Component

1.5

0.4
$\frac{2.42}{2.42}$
0.00

1.4
$\frac{2.99}{2.99}$

2.99

$\frac{1.9}{2.14}$

$\frac{2.14}{2.14}$
0.00

0.00

$0.00 \quad 0.00$

15.0

\begin{tabular}{rrr}
5.9 & 10.3 & 11 \\
2.79 & $\frac{2.24}{1.86}$ & $\frac{2}{1 .}$ \\
\hline 1.87 & 0.20 & 0.2 \\
0.47 & 0.18 & 0.18
\end{tabular}

11.2
2.27
1.89
0.20
0.18

$14.8 \quad 14.1$

$\frac{2.53}{2.04}$

(Amoco Canada (WI))

Total Price (Noyes, Minnesota)

Commodity Componen

Reservation Fee Component

NORTHERN STATES POWER COMPANY

(Canadian Occidental (WI))

AVG Daily Quantity

Total Price (Noyes, Minnesota)

Commodity Component

Demand Component

7.5

$\begin{array}{rrr}7.5 & 10.3 & 7.5 \\ \frac{3.06}{2.26} & \frac{3.68}{2.81} & \frac{2.63}{1.93} \\ 0.67 & 0.74 & 0.57 \\ 0.13 & 0.13 & 0.13\end{array}$

2.2
$\frac{2.32}{2.32}$
0.00

2.1
2.56
2.56

$\frac{2.56}{2.56}$
0.00

2.4
$\frac{2.52}{2.52}$
0.00

0.8
$\frac{2.74}{2.74}$
0.00

0.9
$\frac{2.92}{2.92}$
0.00
0.00

0.7
4.05
4.05
0.00
0.00

\begin{tabular}{r}
0.4 \\
4.35 \\
\hline 4.35 \\
0.00
\end{tabular}

$\begin{array}{r}0.7 \\ 3.56 \\ \hline 3.56\end{array}$

$\frac{3.56}{3.56}$

0.00

$\begin{array}{r}0.7 \\ 4.45 \\ \hline 4.45\end{array}$

4.45

0.00

Reservation Fee Component

(1) Beginning in January 1999, additional volumes were imported under a short-term authorization using various suppliers.
55,045

403,774

$3 Q 2000$

TOTAL MCF

919,989

$2,739,958$

24,948

236,304

688,628

$3,184,883$

447,675

$1,802,272$ 


\section{VOLUME AND PRICE REPORT}

\section{Long-Term Imports}

Volumes (MMcf/d) \& Prices (\$/MMBTU) of Natural Gas Imported During the Past 12 Months 10/01/99 - 09/30/00

\section{Long-Term Importer}

NORTHERN STATES POWER COMPANY

(ProGas Limited (WI))

AVG Daily Quantity

Total Price (Noves, Minnesota)

Commodity Component

Demand Component

Reservation Fee Component $\begin{array}{lllllll}1999 & 1999 & 1999 & 2000 & 2000 & 2000\end{array}$

Vols.

7.5

\begin{tabular}{rrr}
7.5 & 6.3 & 7.5 \\
2.76 & 3.48 & 2.50 \\
\hline 2.31 & $\frac{2.91}{2.04}$ \\
0.45 & 0.57 & 0.46 \\
0.00 & 0.00 & 0.00
\end{tabular}

2000
Jan.

Feb.

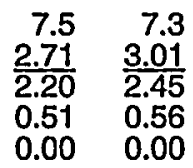

NORTHERN STATES POWER COMPANY (MINNESOTA)

(Amoco Canada (MN))

AVG Daily Quantity

Total Price (Noyes, Minnesota)

Commodity Componen

Demand Component

Reservation Fee Component

16.0

\begin{tabular}{ll}
15.8 & 13.5 \\
2.79 & 3 \\
\hline 2.23 & 2 \\
0.56 & 0 \\
0.00 & 0.07
\end{tabular}

NORTHERN STATES POWER COMPANY (MINNESOTA)

(TCGS Ltd.)

AVG Daily Quantity

Total Price (Noyes, Minnesota)

Commodity Componen

Reservation Fee Component

19.4

19.2
2.60
2.10
0.50
0.00

2000

20002000

$2000 \quad 2000 \quad 2000$

Reservation Fee Component

NORTHERN UTILITIES, INC.

(Renaissance Energy Ltd.)

AVG Daily Quantity

Total Price (Niagara Falls, NY)

Commodity Componen

Demand Component

Reservation Fee Component

\begin{tabular}{rr}
1.0 & 1.0 \\
3.07 & 3.56 \\
\hline 2.23 & 2.69 \\
0.84 & 0.87 \\
0.00 & 0.00
\end{tabular}
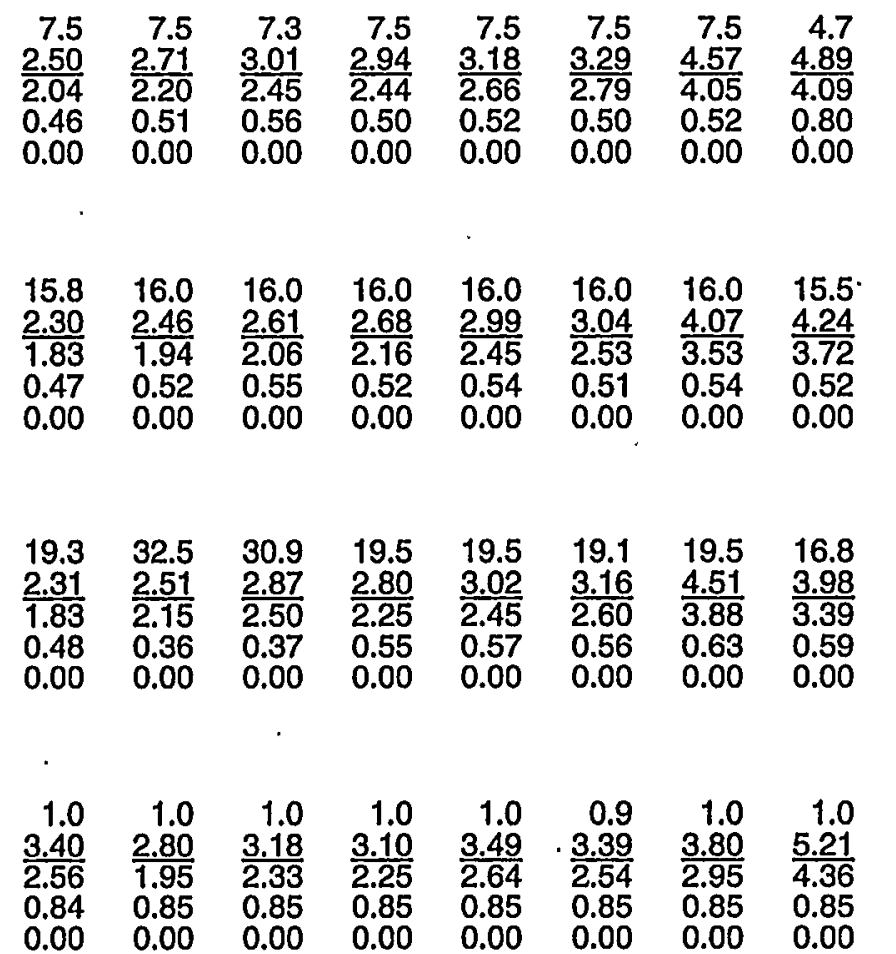

$\begin{array}{r}4.7 \\ 4.39 \\ \hline 3.59\end{array}$

$0.80 \quad 0.80$

\begin{tabular}{r}
4.7 \\
5.08 \\
\hline 4.37 \\
0.71 \\
0.00
\end{tabular}

$\begin{array}{lll}0.00 & 0.00 & 0.00\end{array}$

\section{NORTHWEST NATURAL GAS COMPANY}

(Amoco Canada)

AVG Daily Quantity

Total Price (Sumas, Washington)

Commodily Component

Demand Component

Reservation Fee Component

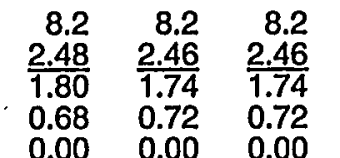

0.00 \begin{tabular}{ll}
14.4 & 19.3 \\
3.45 & 2.31 \\
\hline 2.73 & 1.83 \\
0.72 & 0.48 \\
0.00 & 0.00
\end{tabular}

\begin{tabular}{ll}
13.5 & 15.8 \\
3.21 & 2.30 \\
\hline 2.64 & 1.83 \\
0.57 & 0.47 \\
0.00 & 0.00 \\
& \\
& \\
& \\
14.4 & 19.3 \\
3.45 & 2.31 \\
\hline 2.73 & $\frac{1.83}{1.83}$ \\
0.72 & 0.48 \\
0.00 & 0.00
\end{tabular}

\begin{tabular}{l}
16.0 \\
2.46 \\
\hline 1.94 \\
0.52 \\
0.00
\end{tabular}

\begin{tabular}{l}
16.0 \\
2.61 \\
\hline 2.06 \\
0.55 \\
0.00
\end{tabular}

16.0

16.0

16.0

2.16

0.52
0.00

$\frac{2.99}{2.45}$

2.45
0.54

0.00

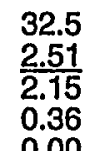

\begin{tabular}{ll}
$30.9 \quad 19.5$ \\
\hline
\end{tabular}

$\frac{2.87}{2.50}$

0.36

0.00

\begin{tabular}{l}
19.5 \\
3.02 \\
\hline 2.45 \\
0.57 \\
0.00
\end{tabular}

19.1
3.16

$\frac{3.16}{2.60}$

0.56

\begin{tabular}{l}
16.0 \\
4.07 \\
\hline 3.53
\end{tabular}

$\frac{4.07}{3.53}$

.51

00 \begin{tabular}{l}
15.5 \\
4.24 \\
\hline 3.72 \\
0.52
\end{tabular}

\begin{tabular}{l}
16.0 \\
3.72 \\
\hline 3.20
\end{tabular}

$\frac{3.72}{3.20}$

0.52
0.00

16.0

$\frac{4.29}{3.76}$

0.53

0.00

$1,453,573$

3Q 2000

TOTAL MCf

YTD

TOTAL MCf

433,890

$1,790,414$

$4,360,704$

90,988

270,986

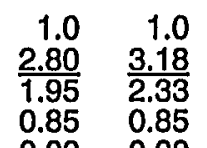

$\begin{array}{r}1.0 \\ 3.10 \\ \hline 2.25\end{array}$

\begin{tabular}{r}
1.0 \\
3.49 \\
\hline 2.64 \\
0.85
\end{tabular}

\begin{tabular}{r}
0.9 \\
3.39 \\
\hline 2.54 \\
0.85
\end{tabular}

1.0
$\frac{3.80}{2.95}$
0.85

1.0
$\frac{5.21}{4.36}$

$\begin{array}{rr}1.0 & 1.0 \\ \frac{4.36}{3.51} & \frac{5.36}{4.51}\end{array}$

$\frac{5.36}{4.51}$

$\begin{array}{lllllllll}0.85 & 0.80 & 0.80 & 0.00 & 0.00 & 0.00 & 0.00 & 0.85 & 0.85\end{array}$

$1,708,705$

$5,979,405$

$4.82 \quad 4.82$

\begin{tabular}{l}
4.82 \\
\hline 4.19 \\
0.63
\end{tabular}

0.00

8.2
2.46

$\frac{2.46}{1.74} \quad \frac{2.46}{1.74} \quad \frac{2.46}{1.74}$

$\begin{array}{lll}1.74 & 1.74 & 1.74 \\ 0.72 & 0.72 & 0.72\end{array}$

0.00 $\frac{2.46}{1.74}$

$\frac{2.46}{1.74}$

0.72

1.74

0.72

0.72

0.00

0.72

0.720 .72

0.00 


\section{Long-Term Importer}

NORTHWEST NATURAL GAS COMPANY (Burlington Resources Canada Energy Ltd.) AVG Daily Quantity

Total Price (Eastport, Idaho)

Commodity Component

Demand Component

Reservation Fee Component

NORTHWEST NATURAL GAS COMPANY (CanWest Gas Supply)

AVG Daily Quantity

Total Price (Sumas, Washington)

Commodity Component

Demand Component

Reservation Fee Component

NORTHWEST NATURAL GAS COMPANY (Engage Energy Canada)

AVG Daily Quantity

Total Price (Eastport, Idaho)

Commodity Component

Demand Component

Reservation Fee Component

NORTHWEST NATURAL GAS COMPANY

(Summit Resources Limited)

AVG Daily Quantity

Total Price (Eastport, Idaho)

Commodity Componen

Demand Component

Reservation Fee Component

\section{NUI CORPORATION (1)}

(TCGS Ltd.)

AVG Daily Quantity

Total Price (Niagara Falls, NY)

Commodity Component

Demand Component

Reservation Fee Component
VOLUME AND PRICE REPORT

\section{Long-Term Imports}

Volumes (MMcf/d) \& Prices (\$MMBTU) of Natural

Gas Imported During the Past 12 Months 10/01/99 - 09/30/00

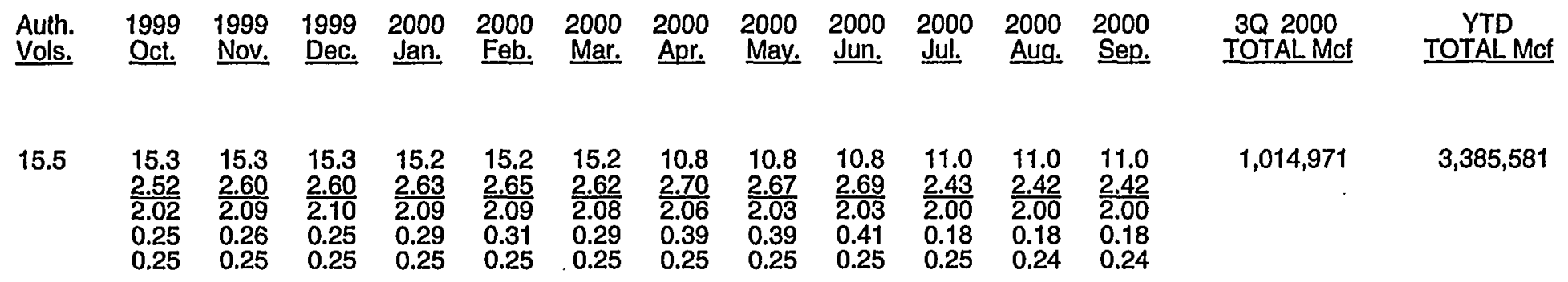

$\begin{array}{llllllllllll}94.7 & 94.6 & 94.7 & 94.3 & 94.3 & 94.2 & 94.3 & 94.3 & 93.9 & 92.1 & 94.3 & 94.3 \\ \frac{2.42}{1.79} & \frac{2.95}{2.26} & \frac{2.31}{1.62} & \frac{2.33}{1.64} & \frac{2.39}{1.70} & \frac{2.35}{1.66} & \frac{2.77}{2.08} & \frac{2.79}{2.09} & \frac{3.68}{2.99} & \frac{4.21}{3.50} & \frac{3.08}{2.39} & \frac{3.49}{2.80} \\ 0.63 & 0.69 & 0.69 & 0.69 & 0.69 & 0.69 & 0.69 & 0.70 & 0.69 & 0.71 & 0.69 & 0.69 \\ 0.00 & 0.00 & 0.00 & 0.00 & 0.00 & 0.00 & 0.00 & 0.00 & 0.00 & 0.00 & 0.00 & 0.00\end{array}$

23.3

$\begin{array}{llllllllllll}13.8 & 13.8 & 13.8 & 13.8 & 13.8 & 13.8 & 13.8 & 13.8 & 13.8 & 13.8 & 13.7 & 13.8 \\ \frac{2.62}{2.10} & \frac{2.69}{2.16} & \frac{2.62}{2.10} & \frac{2.63}{2.08} & \frac{2.64}{2.07} & \frac{2.64}{2.09} & \frac{2.76}{2.20} & \frac{2.81}{2.26} & \frac{3.09}{2.53} & \frac{3.34}{2.79} & \frac{3.12}{2.57} & \frac{3.31}{2.75} \\ 0.26 & 0.27 & 0.26 & 0.29 & 0.31 & 0.29 & 0.30 & 0.29 & 0.30 & 0.29 & 0.29 & 0.30 \\ 0.26 & 0.26 & 0.26 & 0.26 & 0.26 & 0.26 & 0.26 & 0.26 & 0.26 & 0.26 & 0.26 & 0.26\end{array}$

8.0

$\begin{array}{rrr}7.8 & 7.9 & 7.9 \\ \frac{2.45}{2.25} & \frac{2.69}{2.48} & \frac{2.69}{2.49} \\ 0.20 & 0.21 & 0.20 \\ 0.00 & 0.00 & 0.00\end{array}$

7.8
$\frac{2.70}{2.47}$
0.23
0.00

7.8
2.72
2.47
0.25

7.8
2.70
2.47
0.23
0.00

$\frac{5.0}{2.48}$

$\frac{2.48}{2.10}$

0.38

0.00

\begin{tabular}{rr}
5.0 & 5.0 \\
2.48 & 2.46 \\
\hline 2.10 & 2.30
\end{tabular}

$2.10 \quad 2.10$

0.38
0.00 $\quad 0.36$

5.0
$\frac{2.47}{2.11}$
0.36

$\begin{array}{r}5.0 \\ 2.47 \\ \hline 2.10\end{array}$

2.10

0.00
$8,607,037$

$25,755,000$

$1,264,244$

$3,767,191$

461,507

$1,627,246$

183,540

546,630

2.0

$\begin{array}{rrr}2.0 & 1.6 & 2.0 \\ \frac{3.12}{2.42} & \frac{3.79}{2.92} & \frac{2.71}{2.02} \\ 0.70 & 0.87 & 0.69 \\ 0.00 & 0.00 & 0.00\end{array}$

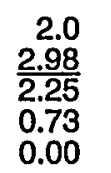

2.0
$\frac{3.31}{2.53}$
0.78
0.00

2.0
3.24
2.51
0.73
0.00

$\frac{3.54}{2.78}$

3.71

$\frac{3.71}{2.98}$

0.73
0.00

2.0
5.02
4.27
0.75
0.00

2.0
4.97
4.24

4.24
0.73

2.0
$\frac{4.36}{3.69}$
0.67

2.0

$\frac{5.22}{4.47}$

4.47
0.75

0.00

(1) Gas supplies NUI's Waverly Gas Service and Valley Cities Gas Service LDC divisions. 


\section{VOLUME AND PRICE REPORT}

\section{Long-Term Imports}

Volumes (MMcf/d) \& Prices (\$MMBTU) of Natural Gas Imported During the Past 12 Months 10/01/99 - 09/30/00

Long-Term Importer

$\begin{array}{llllllllll}\text { Auth. } & 1999 & 1999 & 1999 & 2000 & 2000 & 2000 & 2000 & 2000 & 2000\end{array}$

Vols.

NW ALASKAN PIPELINE CO-Pan-Alberta Gas (I)

(Pan-Alberta Gas Ltd.)

AVG Daily Quaitity

$200.0^{\circ}$

Oct. Nov.

Feb.

Mar.

pr.

$\begin{array}{rrr}199.6 & 149.8 & 213.4 \\ \frac{2.40}{2.16} & \frac{2.85}{252} & \frac{2.03}{1.80}\end{array}$

$\begin{array}{lll}2.16 & 2.52 & 1.80\end{array}$

$\begin{array}{lll}0.24 & 0.33 & 0.23 \\ 0.00 & 0.00 & 0.00\end{array}$
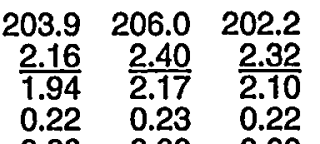
$\begin{array}{r}203.3 \\ 2.57 \\ \hline\end{array}$

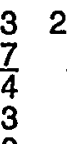

May.

2000

Commodily Compone

Reservation Fee Component

NW ALASKAN PIPELINE CO-Pan-Alberta Gas (II)

(Pan-Alberta Gas Ltd.)

Total Price (Port of Morgan, MT)

Commodity Component

Demand Component

Reservation Fee Component

\section{OCEAN STATE POWER}

(ProGas Limited)

AVG Daily Quantity

Total Price (Niagara Falls, NY)

Commodity Component

Demand Component

Reservation Fee Component

OCEAN STATE POWER II

(ProGas Limited)

AVG Daily Quantity

Total Price (Niagara Falls, NY)

Commodity Component

Demand Component

Reservation Fee Component

\section{OCEAN STATE POWER II}

(TCGS Ltd.)

AVG Daily Quantity

Total Price (Niagara Falls, NY)

Commodily Componen

Demand Component

Reservation Fee Component
600.0

$\begin{array}{rrrrrrrrrrrr}470.9 & 457.7 & 483.5 & 491.7 & 481.9 & 482.4 & 481.4 & 484.2 & 476.1 & 474.9 & 476.3 & 482.7 \\ \frac{2.17}{2.16} & \frac{2.53}{2.52} & \frac{1.81}{1.80} & \frac{1.95}{1.94} & \frac{2.18}{2.17} & \frac{2.11}{2.10} & \frac{2.35}{2.34} & \frac{2.51}{2.50} & \frac{3.70}{3.69} & \frac{3.74}{3.74} & \frac{3.29}{3.29} & \frac{4.02}{4.02} \\ 0.01 & 0.01 & 0.01 & 0.01 & 0.01 & 0.01 & 0.01 & 0.01 & 0.01 & 0.00 & 0.00 & 0.00 \\ 0.00 & 0.00 & 0.00 & 0.00 & 0.00 & 0.00 & 0.00 & 0.00 & 0.00 & 0.00 & 0.00 & 0.00\end{array}$

50.0

$\begin{array}{llllllllllll}50.0 & 50.0 & 50.0 & 50.0 & 50.0 & 50.0 & 50.0 & 50.0 & 50.0 & 50.0 & 50.0 & 50.0 \\ \frac{1.81}{0.88} & \frac{1.80}{0.88} & \frac{1.80}{0.87} & \frac{1.80}{0.80} & \frac{2.33}{1.31} & \frac{2.57}{1.55} & \frac{2.15}{1.14} & \frac{2.77}{1.76} & \frac{2.87}{1.88} & \frac{3.28}{2.28} & \frac{3.14}{2.14} & \frac{3.32}{2.32} \\ 0.93 & 0.92 & 0.93 & 1.00 & 1.02 & 1.02 & 1.01 & 1.01 & 0.99 & 1.00 & 1.00 & 1.00 \\ 0.00 & 0.00 & 0.00 & 0.00 & 0.00 & 0.00 & 0.00 & 0.00 & 0.00 & 0.00 & 0.00 & 0.00\end{array}$

25.0

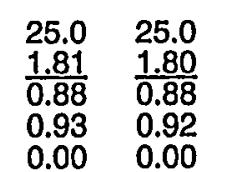

0.00

25.0

\begin{tabular}{ll}
25.0 & 25.0 \\
1.80 & 1.80 \\
\hline 0.87 & 0.80 \\
0.93 & 1.00 \\
0.00 & 0.00
\end{tabular}
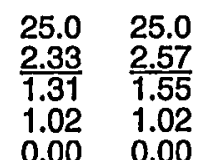

25.0
2.15
1.14

$\begin{array}{r}25.0 \\ .2 .77 \\ \hline\end{array}$

$\begin{array}{llll}25.0 & 25.0 & 25.0 & 25.0 \\ \frac{1.81}{0.94} & \frac{1.80}{0.94} & \frac{1.81}{0.94} & \frac{1.80}{0.90} \\ 0.87 & 0.86 & 0.87 & 0.90 \\ 0.00 & 0.00 & 0.00 & 0.00\end{array}$

0.00 \begin{tabular}{l}
25.0 \\
2.32 \\
\hline 1.41 \\
0.91 \\
0.00
\end{tabular}

0.91 \begin{tabular}{l}
25.0 \\
2.57 \\
\hline 1.63 \\
0.94 \\
0.00
\end{tabular}
1.14
1.01

0.00

1.01
0.00

\begin{tabular}{l}
25.0 \\
2.87 \\
\hline 1.88 \\
0.99
\end{tabular}

\begin{tabular}{l}
25.0 \\
3.29 \\
\hline 2.28 \\
1.01
\end{tabular}

1.01
0.00

\begin{tabular}{l}
25.0 \\
3.14 \\
\hline
\end{tabular}

$\frac{3.14}{2.14}$

$\begin{array}{ll}1.00 \quad 1.00 \\ 0.00 & 0.00\end{array}$

$00 \quad 0.00$
25.

$\frac{2.15}{1.21}$

0.94

0.00 \begin{tabular}{l}
25.0 \\
2.77 \\
\hline 1.83 \\
0.94 \\
0.00
\end{tabular}

0.00
25.0
2.88
1.95
0.93
0.00

0.00

$\begin{array}{lll}25.0 & 25.0 & 25.0 \\ \frac{3.29}{2.35} & \frac{3.14}{2.21} & \frac{3.32}{2.39} \\ 0.94 & 0.93 & 0.93 \\ 0.00 & 0.00 & 0.00\end{array}$

3Q 2000 TOTAL MCf

\section{YTD}

TOTAL Mc

$18,402,385^{\circ} \quad 55,351,494$

$43,967,149$

$131,874,277$

$4,600,000$

$13,700,000$

$2,300,000$

$6,850,000$

$2,300,000$

$6,850,000$ 
VOLUME AND PRICE REPORT

\section{Long-Term Imports}

Volumes (MMcf/d) \& Prices (\$/MMBTU) of Natural

Gas Imported During the Past 12 Months 10/01/99 - 09/30/00

Long-Term Importer

ORANGE AND ROCKLAND UTILITIES, INC.

(Wascana Energy Marketing)

AVG Daily Quantity

Total Price (Niagara Falls, NY)

Commodity Component

Demand Component

Reservation Fee Component

$\begin{array}{lllll}1999 & 1999 & 1999 & 2000 \quad 2000\end{array}$

Oct. Nov. Dec. Jan.

Feb.

2000

2000

20002000200020002000

$3 Q 2000$

TOTAL MCf

YTD

25.0

$\begin{array}{ll}25.0 & 24.9 \\ \frac{3.25}{2.18} & \frac{3.63}{2.48} \\ 0.82 & 0.87 \\ 0.25 & 0.28\end{array}$

24.9
3.53

$\frac{3.53}{2.44}$

25.3

25.3

25.3

25.3

\begin{tabular}{l}
25.3 \\
3.85 \\
\hline
\end{tabular}

$0.25 \quad 0.28$

0.27

$\frac{3.68}{2.48}$

$\frac{3.44}{2.21}$

$.98 \quad 0.91$

$\frac{3.70}{2.48}$

0.94

0.89
0.30

\begin{tabular}{l}
25.3 \\
5.16 \\
\hline 3.79 \\
0.94 \\
0.43
\end{tabular}

\begin{tabular}{lll}
25.3 & 25.3 & 25.3 \\
5.10 & 4.56 & $\frac{5.36}{3.98}$ \\
\hline 3.77 & $\frac{4.29}{3.9}$ & 0.93 \\
0.90 & 0.90 & 0.37 \\
0.43 & 0.37 & 0.45
\end{tabular}

25.0

\begin{tabular}{lllllll}
25.0 & 25.0 & 25.0 & 25.0 & 25.0 & 25.0 & 25.0 \\
$\mathbf{3 . 2 0}$ & $\mathbf{3 . 2 8}$ & $\mathbf{3 . 3 2}$ & $\underline{3.09}$ & $\mathbf{3 . 3 7}$ & $\frac{3.35}{2.35}$ & $\frac{3.50}{2.49}$ \\
\hline 2.27 & $\mathbf{2 . 3 6}$ & $\frac{3.35}{1.98}$ & $\frac{2.35}{2.34}$ & $\mathbf{1}$ \\
0.93 & 0.92 & 0.97 & 1.11 & 1.02 & 1.01 & 1.01
\end{tabular}

25.0
$\frac{3.52}{2.53}$
0.99

$0.00 \quad 0.00$

0.97
0.00

240.0

\begin{tabular}{rr}
242.5 & 232.6 \\
1.87 & 2.38 \\
\hline 1.87 & 2.38 \\
0.00 & 0.00
\end{tabular}

237.6
1.68
1.68
0.00
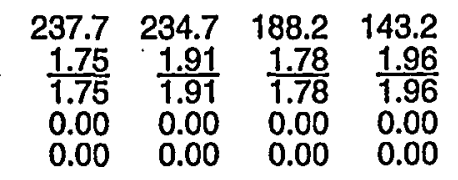

175.8
2.15
2.15
0.00

$\begin{array}{ll}0.00 & 0.00\end{array}$

0.00

8.2

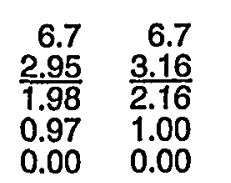

7.2
2.70
1.80
0.90
0.00

\begin{tabular}{r}
5.6 \\
3.05 \\
\hline 1.94 \\
1.11
\end{tabular}

\begin{tabular}{r}
7.5 \\
3.12 \\
\hline 2.06 \\
1.06 \\
0.00
\end{tabular}

\begin{tabular}{r}
5.5 \\
3.37 \\
\hline 2.08 \\
1.29 \\
0.00
\end{tabular}

\begin{tabular}{r}
7.4 \\
3.16 \\
\hline 2.16 \\
1.00 \\
0.00
\end{tabular}

\begin{tabular}{r}
7.2 \\
3.24 \\
\hline 2.27 \\
0.97 \\
0.00
\end{tabular}

\begin{tabular}{r}
7.1 \\
3.94 \\
\hline 2.91 \\
1.03
\end{tabular}

0.00

6.0

\begin{tabular}{rr}
4.7 & 4.7 \\
3.22 & 3.32 \\
\hline 2.25 & 2.32 \\
0.97 & 1.00 \\
0.00 & 0.00
\end{tabular}

\begin{tabular}{r}
5.1 \\
3.03 \\
\hline 2.13 \\
0.90 \\
0.00
\end{tabular}

\begin{tabular}{r}
4.0 \\
3.40 \\
\hline 2.29 \\
1.11 \\
0.00
\end{tabular}

\begin{tabular}{r}
5.2 \\
3.46 \\
\hline 2.40 \\
1.06 \\
0.00
\end{tabular}

\begin{tabular}{r}
3.9 \\
3.70 \\
\hline 2.41 \\
1.29 \\
0.00
\end{tabular}

\begin{tabular}{r}
5.2 \\
3.50 \\
\hline 2.50 \\
1.00 \\
0.00
\end{tabular}

\begin{tabular}{r}
5.1 \\
3.51 \\
\hline 2.54 \\
0.97 \\
0.00
\end{tabular}

\begin{tabular}{r}
5.0 \\
4.17 \\
\hline 3.14 \\
1.03
\end{tabular}

$\begin{array}{ll}3.14 & 3.77\end{array}$

$\begin{array}{ll}1.03 & 0.95 \\ 0.00 & 0.00\end{array}$
$2,324,840$

$6,923,980$

$2,300,000$

$6,850,000$ :

$52,272,331$

687,310

$1,906,479$

486,987

$1,346,256$

(1) Acts as agent for MASSPOWER and Granite State Gas Transmission System.
(2) Successor to NW-Alaskan Pipeline Co; gas sold to SoCal. (See order 1009-A). 


\section{VOLUME AND PRICE REPORT}

\section{Long-Term Imports}

Volumes (MMct/d) \& Prices (\$MMBTU) of Natural

Gas Imported During the Past 12 Months 10/01/99 - 09/30/00

\section{Long-Term Importer}

PITTSFIELD GENERATING CO., L.P.

(Anderson Exploration Ltd.)

AVG Daily Quantity

Total Price (Niagara Falls, NY)

Commodity Componen

Demand Component

Reservation Fee Component .

PITTSFIELD GENERATING CO., L.P.

(Talisman Energy Inc.)

AVG Daily Quantity

Total Price (Niagara Falls, NY)

Commodily Component

Demand Component

Reservation Fee Component

POCO MARKETING LTD. (1)

(IGI Resources, Inc.)

AVG Daily Quantity

Total Price (Sumas, Washington)

Commodity Component

Reservation Fee Component

PORTAL MUNICIPAL GAS (2)

(Sask Energy Inc.)

Total Price (Portal, North Dakota)

Commodity Componen

Demand Component

PROGAS U.S.A. INC. (3)

(ProGas Limited)

AVG Daily Quantity

Total Price (Noyes, Minnesota)

Demand Component

Reservation Fee Component
AVG Daily Quantity

Reservation Fee Component

Commodity Component
Auth. $\quad 1999 \cdot 1999$

Vols.

11.8

$\begin{array}{lll}12.0 & 12.0 & 11.9 \\ \frac{2.79}{1.94} & \frac{2.58}{1.73} & \frac{2.80}{1.95} \\ 0.85 & 0.85 & 0.85\end{array}$

$\begin{array}{lll}0.85 & 0.85 & 0.85\end{array}$

$\begin{array}{lll}0.00 & 0.00 & 0.00\end{array}$

22.4

\begin{tabular}{lll}
23.0 & 22.8 & 22.9 \\
$\frac{2.97}{2.12}$ & $\underline{3.10}$ & $\frac{2.81}{2.25}$ \\
\hline 0.85 & 0.85 & 0.86 \\
0.00 & 0.00 & 0.00
\end{tabular}

$\begin{array}{lll}0.00 & 0.00 & 0.00\end{array}$

20.0

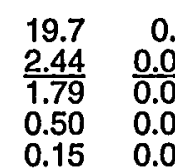

2.3

$\begin{array}{rrr}0.0 & 0.0 & 0.1 \\ \frac{2.72}{2.72} & \frac{2.67}{2.67} & \frac{2.85}{2.85} \\ 0.00 & 0.00 & 0.00 \\ 0.00 & 0.00 & 0.00\end{array}$

85.0

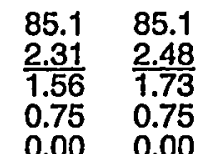

$\begin{array}{ll}0.00 & 0.00\end{array}$

0.0
0.00
0.00
0.00
0.00

0.0
0.00
0.00

0.00
0.00
0.00

$22.4 \quad 22.4$

$\frac{3.11}{2.18}$

0.93

0.93
0.00

$\frac{3.26}{2.33}$

0.93
0.00

22.5
$\frac{3.17}{2.24}$
0.93

\begin{tabular}{l}
22.5 \\
3.24 \\
\hline 2.31
\end{tabular}

0.00

0.00

22.5
3.49
2.57

0.92

\begin{tabular}{r}
22.3 \\
\hline 3.00 \\
0.93
\end{tabular}

0.93
0.00

\begin{tabular}{l}
20.2 \\
3.84 \\
\hline
\end{tabular}

$\frac{3.84}{2.91}$

0.93
0.00

$\frac{3.69}{2.77}$

\section{0}

$\frac{0.00}{0.00}$

0.0
0.00
0.00

0.0

0.0
0.00
0.00

0.00
0.00

$0.00 \quad 0.00$

0.00

0.0
0.00
0.00
0.00
0.00

$\begin{array}{r}0.0 \\ 0.00 \\ \hline 0.00\end{array}$

$\begin{array}{r}0.0 \\ 0.00 \\ \hline 0.00\end{array}$

0.00

$0.00 \quad 0.00$

0.0

$\frac{0.00}{0.00}$

0.00

0.00

\begin{tabular}{|c|c|c|c|c|c|c|c|}
\hline $\begin{array}{l}0.1 \\
2.98 \\
2.98 \\
0.00 \\
0.00\end{array}$ & $\begin{array}{r}0.0 \\
\frac{2.84}{2.84} \\
0.00 \\
0.00\end{array}$ & $\begin{array}{r}0.0 \\
3.22 \\
3.22 \\
0.00 \\
0.00\end{array}$ & $\begin{array}{r}0.0 \\
\cdot 3.41 \\
3.41 \\
0.00 \\
0.00\end{array}$ & $\begin{array}{r}0.0 \\
3.91 \\
3.91 \\
0.00 \\
0.00\end{array}$ & $\begin{array}{r}0.0 \\
3.79 \\
3.79 \\
0.00 \\
0.00\end{array}$ & $\begin{array}{r}0.0 \\
3.80 \\
3.80 \\
0.00 \\
0.00\end{array}$ & $\begin{array}{r}0.0 \\
3.19 \\
3.19 \\
0.00 \\
0.00\end{array}$ \\
\hline
\end{tabular}

2) Volumes often are too small to be shown on a monthly basis.

(3) Supply is resold to ConsumersEnergy Company. Daily conlract volume specified in Order 1197-B. 
VOLUME AND PRICE REPORT

\section{Long-Term Imports}

Volumes (MMcf/d) \& Prices (\$/MMBTU) of Natural

Gas Imported During the Past 12 Months 10/01/99 - 09/30/00

Long-Term Importer

$\begin{array}{lllllllllllll}\text { Auth. } & 1999 & 1999 & 1999 & 2000 & 2000 & 2000 & 2000 & 2000 & 2000 & 2000 & 2000 & 2000\end{array}$

Vols. Oct. Nov. Dec. Jan. Feb. Mar. Apr. May. Jun. Jul. Aug. Sep.

302000

TOTAL MC

YTD

PROGAS U.S.A. INC. (1)

(ProGas Limited)

AVG Daily Quantity

Total Price (Noyes, Minnesota)

Commodity Component

Demand Component

Reservation Fee Component

PROGAS U.S.A. INC. (2)

(ProGas Limited)

AVG Daily Quantity

Total Price (Noyes, Minnesota)

Commodity Componen

Demand Component

Reservation Fee Component

PROGAS U.S.A. INC. (3)

(ProGas Limited)

AVG Dally Quantity

Total Price (Noyes, Minnesota)

Commodity Componen

Demand Component

Reservation Fee Component

PROGAS U.S.A. INC. (4)

(ProGas Limited)

AVG Daily Quantity

Total Price (Port of Morgan, MT)

Commodity Component

Demand Component

Reservation Fee Component
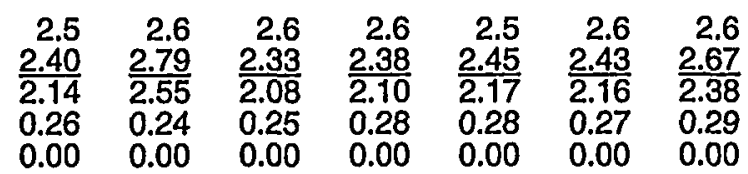

2.08

2.63

\begin{tabular}{r}
2.4 \\
4.11 \\
\hline 3.77 \\
0.34 \\
0.00
\end{tabular}

1.7
4.38

1.9
8.70

2.1
4.17

0.35

0.40

0.40

0.37

3.82

0.35

12.8

$\begin{array}{lll}13.0 & 11.0 & 13.0 \\ \frac{2.77}{2.22} & \frac{3.47}{2.75} & \frac{2.47}{1.88} \\ 0.55 & 0.72 & 0.59\end{array}$

\begin{tabular}{l}
13.0 \\
2.69 \\
\hline 2.04
\end{tabular}

$\frac{2.69}{2.04}$

0.65

$\begin{array}{ll}0.00 & 0.00\end{array}$

0.00

$\begin{array}{ll}0.00 & 0.00\end{array}$

26.5

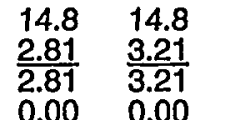

14.8
2.34
2.34
0.00

14.8
2.51
2.51
0.00

14.8
2.76
2.76
0.00

$0.00 \quad 0.00$

0.00

$0.00 \quad 0.00$

14.8
2.73
2.73
0.00

0.00

$14.8 \quad 14.8$

$\frac{3.10}{3.10} \cdot \frac{3.28}{3.28}$

$\begin{array}{ll}3.10 & 3.28 \\ 0.00 & 0.00\end{array}$

$\begin{array}{ll}0.00 & 0.00\end{array}$

\begin{tabular}{l}
14.8 \\
4.41 \\
\hline 4.41
\end{tabular}

$\frac{4.41}{4.41}$

0.00
0.00

$\begin{array}{r}5.6 \\ 4.94 \\ \hline 3.96\end{array}$

$\begin{array}{r}3.5 \\ 5.49 \\ \hline 4.00\end{array}$

6.3

$\frac{4.30}{3.50}$

3.50

1.49

0.00

7.0
5.02

4.28

0.74

0.00

30.0
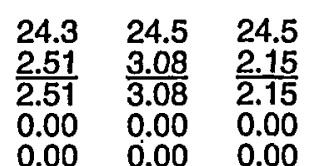

24.3
2.37
2.37
0.00
0.00

24.4
2.62
2.62
0.00
0.00

24.4
2.57
2.57
0.00
0.00

24.2
2.84
2.84
0.00
0.00

24.3

$\frac{3.01}{3.01}$

0.00

\begin{tabular}{l}
24.4 \\
4.26 \\
\hline 4.26
\end{tabular}

$\frac{4.26}{4.26}$

4.25

\begin{tabular}{l}
24.4 \\
4.25 \\
\hline
\end{tabular}

24.4
3.76
3.76

$\frac{3.76}{3.76}$

4.55

4.55

0.00

PROGAS U.S.A. INC. - (DYNEGY)

(ProGas Ltd.)

AVG Daily Quantity

Total Price (Port of Morgan, MT)

Commodity Component

Demand Component

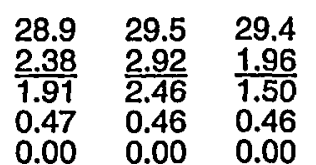

\begin{tabular}{l}
29.1 \\
2.19 \\
\hline 1.73 \\
0.46 \\
0.00
\end{tabular}

29.1
2.43
1.97
0.46
0.00

29.1
2.40
1.94
0.46
0.00

29.0
2.69
2.22
0.47
0.00

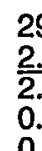

$\begin{array}{lllll}29.0 & 29.1 & 27.8 & 29.2 & 29.2 \\ \frac{2.88}{2.41} & \frac{4.16}{3.69} & \frac{4.21}{3.69} & \frac{3.63}{3.17} & \frac{4.41}{3.95} \\ 0.47 & 0.47 & 0.52 & 0.46 & 0.46 \\ 0.00 & 0.00 & 0.00 & 0.00 & 0.00\end{array}$

$2,642,904$

(1) Supplies are resold to the city of Perham, MN. Dally contract quantity increased from 2.3 to 2.6 MMcf in Order 1197-B.

2. Supplies are resold to Great Plains Natural Gas Company.

(4) Each quarter approximately $40 \%$ is delivered to Columbia at Ventura, IA and $60 \%$ is delivered to Tenaska at Harper, IA. 


\section{VOLUME AND PRICE REPORT}

Long-Term Imports

Volumes (MMct/d) \& Prices (\$MMBTU) of Natural

Gas Imported During the Past 12 Months 10/01/99 - 09/30/00

Long-Term Importer

Auth

PROGAS U.S.A. INC. - (TENASKA GAS COMPANY)

(ProGas Limited)

AVG Daily Quantity

Total Price (Port of Morgan, MT)

Commodity Component

Demand Component

Reservation Fee Component

PROGAS U.S.A., INC (1)

(Progas U.S.A. Inc.)

AVG Daily Quantity

Total Price (Waddington, NY)

Commodity Component

Demand Component

Reservation Fee Component

PROGAS U.S.A., INC (2)

(Progas U.S.A. Inc.)

AVG Daily Quantily

Total Price (Eastport, Idaho)

Commodity Componen

Demand Component

Reservation $\mathrm{Fee}$ Component

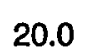

\begin{tabular}{lllll}
29.0 & 28.9 & 28.9 & 32.6 & 29.4 \\
2.73 & $\frac{3.37}{3.37}$ & $\frac{2.61}{2.61}$ & $\frac{3.40}{3.40}$ & \\
\hline 2.73 & 3.3 \\
0.00 & 0.00 & 0.00 & 0.00 & 0 \\
0.00 & 0.00 & 0.00 & 0.00 & 0.0
\end{tabular}

29.2

$\frac{3.31}{3.31}$

$0.00 \quad 0.00$

$\begin{array}{ll}19.6 & 19 . \\ 1.96 & 2.46 \\ 0.50 & 0.56 \\ 0.00 & 0.0\end{array}$

19.4
2.19
1.69
0.50
0.00

19.4
$\frac{2.43}{1.93}$
0.50

2.40

$\frac{2.40}{1.90}$

0.50
0.00

2000

2000
May.

2000

2000

7.8

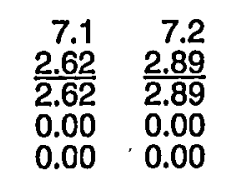

7.2
2.58
2.58
0.00
0.00

7.2
2.52
2.52
0.00
0.00

7.2
$\frac{2.54}{2.54}$
0.00
0.00

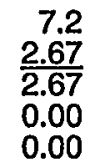

7.2
2.89
2.89
0.00

$\begin{array}{lr}7.2 & 7.2 \\ 9.9 & \frac{3.30}{3.30} \\ 0.00 & 0.00\end{array}$

3.2
30
.00
.00

7.2
4.18
4.18
0.00
0.00

\begin{tabular}{r}
7.2 \\
3.95 \\
\hline 3.95 \\
0.00
\end{tabular}

$\frac{4.4}{4.4}$

$7.2 \quad 7.1$

$\frac{4.41}{4.41} \quad \frac{5.33}{5.33}$

PROJECT ORANGE ASSOCIATES, L.P. (3)

(Noranda, Inc.)

AVG Daily Quantity

Total Price (Niagara Falls, NY)

Commodity Component

Commodity Compone

Reservation Fee Component

0.0

\begin{tabular}{|c|c|c|c|c|c|c|c|c|c|c|c|}
\hline $\begin{array}{l}10.9 \\
3.23 \\
0.73 \\
2.50 \\
0.00\end{array}$ & $\begin{array}{l}14.0 \\
2.92 \\
0.73 \\
2.19 \\
0.00\end{array}$ & $\begin{array}{l}17.3 \\
2.85 \\
0.73 \\
2.12 \\
0.00\end{array}$ & $\begin{array}{l}19.9 \\
2.57 \\
0.73 \\
1.84 \\
0.00\end{array}$ & $\begin{array}{l}17.2 \\
2.78 \\
0.73 \\
2.05 \\
0.00\end{array}$ & $\begin{array}{l}20.3 \\
2.56 \\
0.73 \\
1.83 \\
0.00\end{array}$ & $\begin{array}{l}20.3 \\
3.09 \\
0.73 \\
2.36 \\
0.00\end{array}$ & $\begin{array}{l}20.3 \\
.3 .09 \\
0.73 \\
2.36 \\
0.00\end{array}$ & $\begin{array}{l}20.3 \\
3.17 \\
0.73 \\
2.44 \\
0.00\end{array}$ & $\begin{array}{r}3.3 \\
3.97 \\
3.97 \\
0.00 \\
0.00\end{array}$ & $\begin{array}{r}0.0 \\
0.00 \\
0.00 \\
0.00 \\
0.00\end{array}$ & $\begin{array}{r}0.0 \\
0.00 \\
0.00 \\
0.00 \\
0.00\end{array}$ \\
\hline
\end{tabular}

\section{PUBLIC SERVICE ELECTRIC \& GAS}

(Alberta NE Gas (TCGS Ltd. 1))

AVG Daily Quantity

Total Price (Waddington, NY)

Commodity Componen

Demand Component

Reservation $\mathrm{Fee}$ Component

\begin{tabular}{rrr}
10.0 & 8.0 & 9.8 \\
2.80 & 3.22 & 2.36 \\
\hline 2.01 & 2.43 & 1.57 \\
0.79 & 0.79 & 0.79 \\
0.00 & 0.00 & 0.00
\end{tabular}

10.0
$\frac{2.52}{1.66}$
0.86
0.00

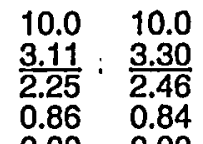

$\begin{array}{ll}0.86 & 0.84 \\ 0.00 & 0.00\end{array}$

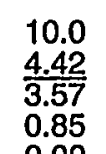

10.0
$\frac{4.42}{3.57}$
0.85
0.00

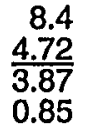

$\frac{4.72}{3.87}$

0.85
0.00
$10.0 \quad 10.0$

$\frac{3.93}{3.08} \quad \frac{4.83}{3.08}$

$3.08 \quad 3.98$ \begin{tabular}{ll}
$0.00 \quad 0.00$ \\
\hline
\end{tabular}

(1) Supplies are marketed in the Northeast, primarily in New York State. Dally contract quantily increase from 16.4 to 29.4 approved in Order 1206-A.

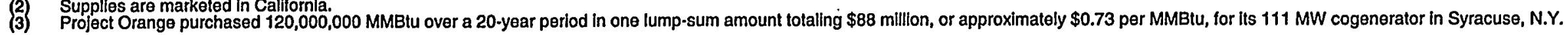




\section{Long-Term Importer}

\section{PUGET SOUND ENERGY, INC.}

\section{(Amoco Canada)}

AVG Daily Quantity

Total Price (Sumas, Washington)

Commodity Compone

Demand Component

Reservation Fee Component

\section{PUGET SOUND ENERGY, INC.}

\section{(Duke Energy)}

AVG Daily Quantity

Total Price (Sumas, Washington)

Commodity Component

Demand Component

Reservation $\mathrm{Fee}$ Component

PUGET SOUND ENERGY, INC.

(Engage Energy Canada)

AVG Daily Quantity

Total Price (Sumas, Washington)

Commodity Component

Demand Component

Reservation $\mathrm{Fee}$ Component

PUGET SOUND ENERGY, INC.

\section{(Engage Energy Canada)}

AVG Daily Quantity

Total Price (Eastport, Idaho)

Commodity Component

Demand Component

Reservation Fee Component

PUGET SOUND ENERGY, INC.

(Poco Petroleums Ltd.)

AVG Daily Quantity

Total Price (Sumas; Washington)

Commodity Compone

Reservation Fee Component
VOLUME AND PRICE REPORT

Long-Term Imports

Volumes (MMct/d) \& Prices (\$MMBTU) of Natural

Gas Imported During the Past 12 Months 10/01/99 - 09/30/00 $\begin{array}{llllllllllllll}\text { Auth. } & 1999 & 1999 & 1999 & 2000 & 2000 & 2000 & 2000 & 2000 & 2000 & 2000 & 2000 & 2000 & 3 Q\end{array}$

Vols. Oct. Nov. Dec. Jan. Feb. Mar. Apr. May. Jun. Jul. $\underline{\text { Aug. Sep. }}$ IOTAL Mcf

YTD

TOTAL MCf

24.0

$\begin{array}{llllllllllll}24.2 & 24.0 & 23.9 & 21.7 & 25.0 & 14.5 & 21.0 & 23.4 & 24.8 & 24.8 & 25.0 & 25.0 \\ \frac{2.54}{1.81} & \frac{2.95}{2.19} & \frac{2.31}{1.57} & \frac{2.32}{1.51} & \frac{2.28}{1.53} & \frac{2.33}{1.53} & \frac{2.76}{1.91} & \frac{2.76}{2.00} & \frac{3.49}{2.66} & \frac{4.10}{3.27} & \frac{3.06}{2.29} & \frac{3.48}{2.61} \\ 0.73 & 0.76 & 0.74 & 0.81 & 0.75 & 0.80 & 0.85 & 0.76 & 0.83 & 0.83 & 0.77 & 0.87 \\ 0.00 & 0.00 & 0.00 & 0.00 & 0.00 & 0.00 & 0.00 & 0.00 & 0.00 & 0.00 & 0.00 & 0.00\end{array}$

14.5

$\begin{array}{llllllllllll}14.5 & 14.6 & 14.5 & 15.0 & 15.0 & 15.0 & 15.0 & 15.0 & 15.0 & 15.0 & 15.0 & 15.0 \\ \frac{2.54}{2.00} & \frac{2.96}{2.42} & \frac{2.31}{1.78} & \frac{2.33}{1.79} & \frac{2.36}{1.75} & \frac{2.34}{1.79} & \frac{2.76}{2.18} & \frac{2.77}{2.23} & \frac{3.67}{3.10} & \frac{4.10}{3.56} & \frac{2.98}{2.40} & \frac{3.48}{2.88} \\ 0.54 & 0.54 & 0.53 & 0.54 & 0.61 & 0.55 & 0.58 & 0.54 & 0.57 & 0.54 & 0.58 & 0.60 \\ 0.00 & 0.00 & 0.00 & 0.00 & 0.00 & 0.00 & 0.00 & 0.00 & 0.00 & 0.00 & 0.00 & 0.00\end{array}$

9.6

$\begin{array}{rrrlllllllll}9.6 & 19.8 & 4.8 & 14.1 & 20.0 & 19.5 & 18.5 & 17.9 & 20.0 & 20.0 & 20.0 & 20.0 \\ \frac{2.64}{1.69} & \frac{2.96}{2.32} & \frac{2.31}{1.87} & \frac{2.36}{1.72} & \frac{2.41}{1.72} & \frac{2.36}{1.72} & \frac{2.74}{2.09} & \frac{2.78}{2.15} & \frac{3.66}{3.02} & \frac{4.09}{3.47} & \frac{3.06}{2.44} & \frac{3.44}{2.80} \\ 0.65 & 0.64 & 0.44 & 0.64 & 0.69 & 0.64 & 0.65 & 0.63 & 0.64 & 0.62 & 0.62 & 0.64 \\ 0.00 & 0.00 & 0.00 & 0.00 & 0.00 & 0.00 & 0.00 & 0.00 & 0.00 & 0.00 & 0.00 & 0.00\end{array}$

95.8

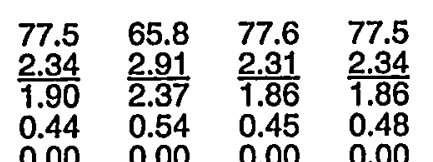

77.9
$\frac{2.40}{1.91}$
0.49

77.9
2.42
1.95
0.47

78.0
2.60
2.12
0.48
0.00

78.0
.2 .63
2.16
0.47
0.00

$\frac{77.5}{2.76}$

$\begin{array}{ll}0.47 & 0.49 \\ 0.00 & 0.00\end{array}$

\begin{tabular}{l}
78.0 \\
3.55 \\
\hline 3.08 \\
0.47 \\
0.00
\end{tabular}

\begin{tabular}{l}
77.4 \\
2.96 \\
\hline 2.48 \\
0.48 \\
0.00
\end{tabular}

77.9
3.24

$\frac{3.24}{2.76}$

0.48
0.00

14.4

$\begin{array}{lllll}14.5 & 15.0 & 14.5 & 15.0 & 14.9 \\ \frac{2.54}{1.86} & \frac{2.83}{2.21} & \frac{2.31}{1.74} & \frac{2.33}{1.69} & \frac{2.48}{1.83} \\ 0.68 & 0.62 & 0.57 & 0.64 & 0.65 \\ 0.00 & 0.00 & 0.00 & 0.00 & 0.00\end{array}$

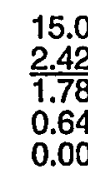

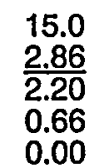

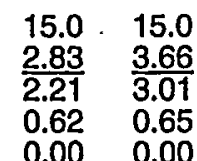

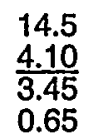

$\frac{4.10}{3.45}$

0.65

0.00
$2,295,325$

$6,245,442$

$1,380,000$

$4,110,000$

$1,840,000$

$5,170,920$

$7,154,664$

$21,313,672$

$1,364,940$

$4,092,240$ 


\section{VOLUME AND PRICE REPORT}

\section{Long-Term Imports}

Volumes (MMcf/d) \& Prices (\$/MMBTU) of Natural Gas Imported During the Past 12 Months 10/01/99-09/30/00

Long-Term Importer

\section{Auth.}

\section{RDO FOODS CO.}

(ProGas Limited)

AVG Daily Quantity

Total Price (Noves, Minnesota)

Commodity Component

Demand Component

Reservation Fee Component

RENAISSANCE ENERGY (U.S.) INC. (1)

(Renaissance Energy Limited)

AVG Daily Quantity

Total Price (Port of Morgan, MT)

Commodity Component

Demand Component

Reservation Fee Component

RENAISSANCE ENERGY (U.S.) INC. (2)

(Renaissance Energy Limited)

AVG Daily Quantity

Total Price (Niagara Falls, NY)

Commodity Componen

Reservation Fee Component

RENAISSANCE ENERGY (U.S.) INC. (3)

(Renaissance Energy Limited)

AVG Daily Quantity

Total Price (Niagara Falls, NY)

Commodity Componen

Demand Component
Reservation Fee Component

1.4

$.4 \quad 0.0 \quad 0.0$

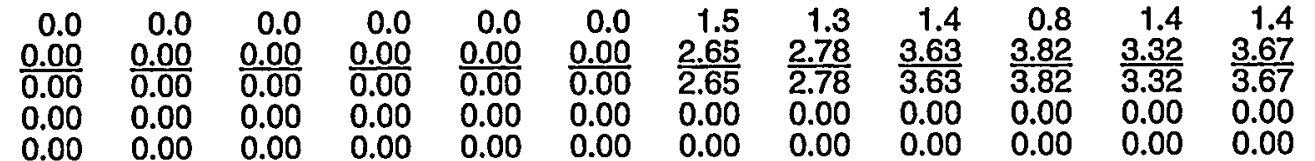

5.0

$\begin{array}{rrrrrrrrrrrr}5.2 & 5.1 & 5.1 & 5.1 & 5.1 & 5.1 & 5.1 & 5.2 & 5.1 & 5.1 & 5.1 & 5.1 \\ \frac{2.18}{2.18} & \frac{2.77}{2.77} & \frac{1.90}{1.90} & \frac{2.11}{2.11} & \frac{2.19}{2.19} & \frac{2.22}{2.22} & \frac{2.24}{2.24} & \frac{3.06}{3.06} & \frac{3.83}{3.83} & \frac{3.84}{3.84} & \frac{3.62}{3.62} & \frac{4.27}{4.27} \\ 0.00 & 0.00 & 0.00 & 0.00 & 0.00 & 0.00 & 0.00 & 0.00 & 0.00 & 0.00 & 0.00 & 0.00 \\ 0.00 & 0.00 & 0.00 & 0.00 & 0.00 & 0.00 & 0.00 & 0.00 & 0.00 & 0.00 & 0.00 & 0.00\end{array}$

469,664

$1,404,771$

2.8

$\begin{array}{rrr}2.8 & 2.8 & \\ \frac{3.21}{2.37} & \frac{3.66}{2.81} & 3 \\ 0.84 & 0.85 & 0.8 \\ 0.00 & 0.00 & 0.0\end{array}$

$\begin{array}{rr}2.8 & 3.20 \\ 2.37 & 2.98 \\ 0.83 & 0.98 \\ 0.00 & 0.00\end{array}$

2.8
2.98
2.05
0.93

$\begin{array}{rrr}2.8 & 2.7 & 2.8 \\ 3.31 & 3.16 & 3.55\end{array}$

$\frac{3.31}{2.32}$
0.99

\begin{tabular}{lr}
3.7 & 2.8 \\
\hline 2.21 & $\frac{3.55}{2.61}$
\end{tabular}

$\begin{array}{r}2.8 \\ 3.73 \\ \hline 2.82\end{array}$

$\begin{array}{r}2.8 \\ 5.11 \\ \hline\end{array}$

$\begin{array}{r}2.8 \\ 4.04 \\ \hline 3.13\end{array}$

$\begin{array}{lllll}0.95 & 0.94 & 0.91 & 0.94 & \mathbf{3 . 1 3}\end{array}$

0.94
0.00

\begin{tabular}{r}
2.7 \\
4.04 \\
\hline 3.11 \\
0.93
\end{tabular}

$\begin{array}{r}2.8 \\ 4.12 \\ \hline 3.18\end{array}$

$0.93 \quad 0.94$

$\begin{array}{lll}0.00 & 0.00 & 0.00\end{array}$

0.00

0.00

\begin{tabular}{rr}
0.0 & 13.3 \\
0.00 & $\frac{3.12}{3.12}$ \\
\hline 0.00 & 3.00 \\
0.00 & 0.00 \\
0.00 & 0.00
\end{tabular}

15.2
2.40
2.40
0.00
0.00

\begin{tabular}{l}
19.2 \\
3.50 \\
\hline 3.50 \\
0.00 \\
0.00
\end{tabular}

12.9

$\begin{array}{rrr}10.3 & 0.0 & 0.0 \\ 3.05 & 0.00 & .0 .00\end{array}$

$\frac{4.70}{4.70}$

$\frac{3.05}{3.05}$

$\frac{0.00}{0.00}$

$\frac{0.0}{0.00}$

0.0
0.00
0.00

$\frac{0.00}{0.00}$

0.0
0.00
0.00

0.00
0.00

0.00

$\begin{array}{ll}00 & 0.00\end{array}$

$\begin{array}{llll}0.00 & 0.00 & 0.00 & 0.00 \\ 0.00 & 0.00 & 0.00 & 0.00\end{array}$

ROCK-TENN COMPANY, MILL DIV., INC. (4)

(Wascana Energy Inc.)

AVG Daily Quantity

Total Price (Highgate Spr., VT)

Commodity Component

Demand Component

2.2

\begin{tabular}{lr}
2.1 & 2.2 \\
3.24 & 3.75 \\
\hline 2.36 & 2.83 \\
0.88 & 0.92 \\
0.00 & 0.00
\end{tabular}

\begin{tabular}{r}
2.2 \\
3.01 \\
\hline 2.16 \\
0.85 \\
0.00
\end{tabular}

\begin{tabular}{r}
2.0 \\
3.13 \\
\hline 2.24 \\
0.89
\end{tabular}

1.8
3.14

2.2
3.22

$\begin{array}{r}2.2 \\ 3.66 \\ \hline 2.69\end{array}$

$\begin{array}{r}1.8 \\ 3.72 \\ \hline 2.79\end{array}$

\begin{tabular}{r}
0.9 \\
4.59 \\
\hline 3.62 \\
0.97
\end{tabular}

2.2
$\frac{4.81}{3.82}$

0.89

$0.89 \quad 0.91$

$\begin{array}{ll}0.00 & 0.00\end{array}$

$\begin{array}{lll}0.97 & 0.99 & 0.95 \\ 0.00 & 0.00 & 0.00\end{array}$

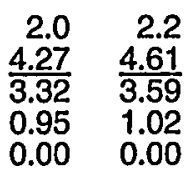

192,240

522,720

(1) Renaissance resells these volumes to AmGas Inc., for final sale to various customers in lowa.

(2) Renalssance resells these volumes to Delmana Power \& Light Company.

(3) Gupplles are sold to markets off the pipeline system of Nationtil 


\section{VOLUME AND PRICE REPORT}

\section{Long-Term Imports}

Volumes (MMct/d) \& Prices (\$/MMBTU) of Natural

Gas Imported During the Past 12 Months 10/01/99 - 09/30/00

\section{Long-Term Importer}

SARANAC POWER PARTNERS, L.P.

(Shell Canada Limited)

AVG Daily Quantity

Commodity Component

Demand Component

Reservation Fee Component

\section{SELKIRK COGEN PARTNERS, L.P.}

(Imperial Oil Resources Limited)

AVG Daily Quantity

Total Price (Waddington, NY)

Commodity Componen

Demand Component

Reservation Fee Component

\section{SELKIRK COGEN PARTNERS, L.P.}

(PanCanadian Petroleum Ltd.)

AVG Daily Quantity

Total Price (Waddington, NY)

Commodity Component

Demand Component

Reservation Fee Component

SELKIRK COGEN PARTNERS, L.P. (1)

(Paramount Resources Ltd.)

AVG Daily Quantity

Total Price (Waddington, NY)

Commodity Component

Demand Component

Reservation Fee Component

\section{SELKIRK COGEN PARTNERS, L.P.}

(Producers Marketing, Ltd.)

AVG Daily Quantity

Total Price (Waddington, NY)

Commodity Componen

Demand Component

Reservation Fee Component $\begin{array}{lll}\text { Auth. } & 1999 & 1999 \\ \text { Vols. } & \text { Oct. } & \text { Nov. }\end{array}$

54.0

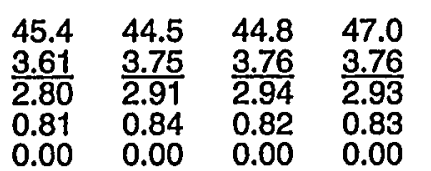

$0.00 \quad 0.00$

0.00

19.0

$\begin{array}{llllllllllll}19.0 & 19.0 & 19.0 & 19.0 & 19.0 & 19.0 & 19.6 & 17.8 & 19.6 & 19.0 & 17.8 & 19.6 \\ \frac{2.57}{1.52} & \frac{2.77}{1.71} & \frac{2.49}{1.43} & \frac{2.66}{1.54} & \frac{2.83}{1.71} & \frac{3.40}{2.29} & \frac{3.54}{2.45} & \frac{3.10}{2.03} & \frac{3.42}{2.34} & \frac{3.51}{2.36} & \frac{3.37}{2.22} & \frac{3.61}{2.46} \\ 1.05 & 1.06 & 1.06 & -1.12 & 1.12 & 1.11 & 1.09 & 1.07 & 1.08 & 1.15 & 1.15 & 1.15 \\ 0.00 & 0.00 & 0.00 & 0.00 & 0.00 & 0.00 & 0.00 & 0.00 & 0.00 & 0.00 & 0.00 & 0.00\end{array}$

19.0

$$
\begin{array}{ll}
19.0 & 19.0 \\
2.58 & 2.77 \\
\hline 1.54 & 1.73 \\
1.04 & 1.04 \\
0.00 & 0.00
\end{array}
$$

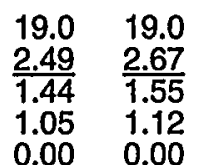

19.0
$\frac{2.85}{1.73}$
1.12
0.00

$\begin{array}{ll}19.0 & 19.6 \\ \frac{3.45}{2.33} & \frac{3.57}{2.48} \\ 1.12 & 1.09 \\ 0.00 & 0.00\end{array}$

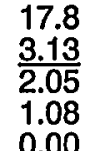

19.6

$\frac{3.46}{2.37}$

19.0
3.55

0.00

16.4

$$
\begin{array}{ll}
15.1 & 15.1 \\
\hline 3.26 & 3.76 \\
\hline 2.38 & 2.88 \\
0.88 & 0.88 \\
0.00 & 0.00
\end{array}
$$

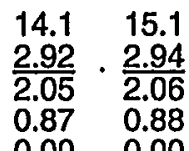

\begin{tabular}{l}
15.1 \\
3.09 \\
\hline 2.20 \\
0.89
\end{tabular}

\begin{tabular}{l}
15.1 \\
3.24 \\
\hline 2.35 \\
0.89
\end{tabular}

\begin{tabular}{l}
15.1 \\
3.59 \\
\hline 2.71 \\
0.88 \\
0.00
\end{tabular}

15.1
.3 .69
2.82
0.87
0.00

\begin{tabular}{l}
15.3 \\
4.71 \\
\hline 3.85 \\
0.86 \\
0.00
\end{tabular}

\begin{tabular}{l}
14.6 \\
4.79 \\
\hline 3.93 \\
0.86 \\
0.00
\end{tabular}

$\begin{array}{r}15.1 \\ 4.03 \\ \hline 3.17\end{array}$

3.17

0.86

\begin{tabular}{ll}
17.8 & 19.6 \\
3.40 & 3.65 \\
\hline .24 & 2.49 \\
1.16 & 1.16 \\
0.00 & 0.00
\end{tabular}

17.0

$\begin{array}{llll}16.9 & 16.9 & 16.9 & 16.9 \\ \frac{2.51}{1.56} & \frac{2.69}{1.74} & \frac{2.42}{1.47} & \frac{2.58}{1.57} \\ 0.95 & 0.95 & 0.95 & 1.01 \\ 0.00 & 0.00 & 0.00 & 0.00\end{array}$

\begin{tabular}{l}
16.9 \\
2.75 \\
\hline 1.74 \\
1.01 \\
0.00
\end{tabular}

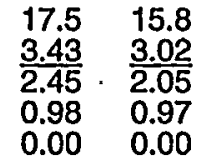

17.5

$\frac{3.32}{2.34}$

2.34
0.98

0.98
0.00 \begin{tabular}{l}
16.9 \\
3.41 \\
\hline 2.36 \\
1.05
\end{tabular}

2.41
1.05
0.00

$15.8 \quad 17.5$

$3.27 \quad 3.51$

$\frac{3.51}{2.46}$

1.05

0.00

(1) As a result of restructuring its power sales agreement with Nlagara Mohawk Power Corp., Selkirk's daily contract demand was reduced to 16.4 MMct, effective 8/31/98. 
VOLUME AND PRICE REPORT

Long-Term Imports

Volumes (MMct/d) \& Prices (\$/MMBTU) of Natural

Gas Imported During the Past 12 Months 10/01/99 - 09/30/00

Long-Term Importer

SIERRA PACIFIC POWER COMPANY (1)

(Amoco Canada)

AVG Daily Quantity

Total Price (Eastport, Idaho)

Commodity Component

Demand Component

Reservation Fee Component

Auth. $\quad 1999 \quad 1999 \quad 1999 \quad 2000$

Vols.

SITHE/NDEPENDENCE POWER PARTNERS, L.P.

(Enron Power Services Inc.)

AVG Daily Quantity

Total Price (Grand Island, NY)

Commodity Componen

Demand Component

Reservation Fee Component

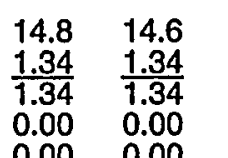

14.8
$\frac{1.34}{1.34}$
0.00
0.00

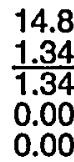

14.8
1.3
1.34
0.00
0.00

200020002000

20002000

$200020002000^{\circ}$

3Q 2000

TOTAL Mcf

TOTAL

0.00

\section{0}

$\frac{4.15}{3.38}$
0.77

\begin{tabular}{ll}
28.0 & 28 \\
4.18 & 4. \\
\hline 3.37 & 3.37 \\
0.81 & 0.79
\end{tabular}

$\begin{array}{lll}0.00 & 0.00 & 0.00\end{array}$

\begin{tabular}{l}
28.0 \\
3.15 \\
\hline 2.36 \\
0.79 \\
0.00
\end{tabular}

\begin{tabular}{l}
28.0 \\
2.98 \\
\hline 2.14 \\
0.84 \\
0.00
\end{tabular}

\begin{tabular}{l}
28.0 \\
2.71 \\
\hline 1.92 \\
0.79 \\
0.00
\end{tabular}

28.0
2.84

$\frac{2.84}{2.03}$

0.81

0.00

\begin{tabular}{ll}
15.0 & 15.0 \\
2.44 & 2.87 \\
\hline 2.44 & 2.87 \\
0.00 & 0.00
\end{tabular}

15.0
2.00
2.00
0.00

$15.0 \quad 15.0$

15.0
$\frac{2.41}{2.41}$

0.00

$0.00 \quad 0.00$

2.17

0.00

2.41

15.0

$\frac{2.39}{2.39}$

Reservation Fee Component

SOUTHERN CONNECTICUT GAS

(Alberta NE Gas (AEC Oil \& Gas Company))

0.00

0.00
0.00

$\begin{array}{r}15.0 \\ 2.76 \\ \hline 2.76\end{array}$

2.76
2.76
0.00

0.00
0.00

15.0
2.94
2.94

$\frac{2.94}{2.94}$
0.00

0.00
0.00

15.0

$\frac{4.07}{4.07}$

0.00
0.00

$\begin{array}{r}15.0 \\ 4.37 \\ \hline 4.37\end{array}$

$\frac{4.37}{4.37}$

0.00
0.00

15.0
3.58

$\frac{3.58}{3.58}$

0.00
0.00

15.0

$\frac{4.47}{4.47}$

0.00

0.00

\section{SOUTHERN CONNECTICUT GAS}

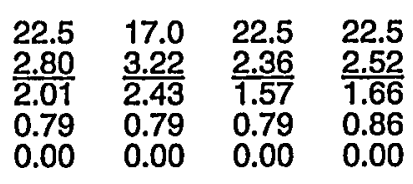

AVG Daily Quantity

Total Price (Waddington, NY)

Commodity Component

Demand Component

Reservation Fee Component

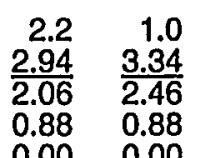

2.2
2.67
1.79
0.88
0.00

\begin{tabular}{r}
2.2 \\
2.97 \\
\hline 2.03 \\
0.94 \\
0.00
\end{tabular}

$\frac{3.32}{2.38}$

(Alberta NE Gas (TCGS Ltd. 1))

AVG Daily Quantity

Total Price (Waddington, NY)

Commodity Component

Demand Component

Reservation Fee Component

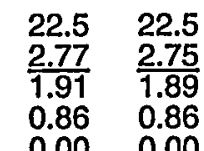

\begin{tabular}{l}
22.5 \\
2.75 \\
\hline 1.89 \\
0.86 \\
0.00
\end{tabular}

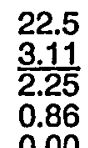

\begin{tabular}{l}
22.5 \\
3.30 \\
\hline 2.46 \\
0.84 \\
0.00
\end{tabular}

$0.00 \quad 0.00$

199,640

462,210

(1) This authorization explred on $3 / 31 / 00$. 


\section{VOLUME AND PRICE REPORT}

\section{Long-Term Imports}

Volumes (MMcf/d) \& Prices (\$/MMBTU) of Natural

Gas Imported During the Past 12 Months 10/01/99 - 09/30/00

\section{Long-Term Importer}

THE U.S. GENERAL SERVICES ADMIN. (1)

(Chief Mountain Natural Gas Coop.)

AVG Daily Quantity

Total Price (Babb, Montana)

Commodity Component

Demand Component

Reservation Fee Component

\section{TM STAR FUEL COMPANY}

(CanWest Gas Supply)

AVG Daily Quantily

Total Price (Sumas, Washington)

Commodity Componen

Demand Component

Reservation Fee Component

TRANSCANADA GAS SERVICES INC.

(TransCanada Gas Services)

AVG Daily Quantity

Total Price (Piltsburg. NH)

Commodity Component

Demand Component

Reservation Fee Component

TRANSCANADA GAS SERVICES INC. (2)

(Yankee Gas Services)

AVG Daily Quantity

Total Price (Waddington, NY)

Commodity Componen

Demand Component

Reservation Fee Component

\section{Auth}

Auth.
Vols.

$$
\begin{array}{r}
0.0 \\
3.26 \\
0.26
\end{array}
$$

$\begin{array}{ll}0.00 & 0.00\end{array}$
2000

Jan.
2000

Feb.
20002000

Mar.
20002000

\author{
(n)
}

TRANSCO ENERGY MARKETING COMPANY (3)

(Canstates Gas Marketing)

AVG Daily Quantity

Total Price (Niagara Falls, NY)

Commodity Component

Demand Component
Reservation Fee Component

48.4

30.1

$$
\begin{array}{lr}
15.6 & 4.8 \\
2.62 & 2.49 \\
\hline 2.62 & 2.49 \\
0.00 & 0.00 \\
0.00 & 0.00
\end{array}
$$

3.4
2.49
2.49
0.00
0.00
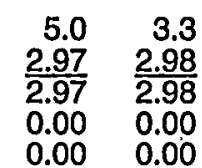

$\begin{array}{r}3.4 \\ 3.00 \\ \hline .00\end{array}$

0.00 .

68.5

\begin{tabular}{rrrrr}
0.0 & 0.0 & 49.4 & 49.4 & 49.4 \\
0.00 & 0.00 & 2.35 & 2.56 & 2.87 \\
\hline 0.00 & 0.00 & $\frac{0.58}{1.51}$ & $\frac{1.71}{1.96}$ \\
0.00 & 0.00 & 0.77 & 0.85 & 0.91 \\
0.00 & 0.00 & 0.00 & 0.00 & 0.00
\end{tabular}

0.00
0.00

3.7
$\frac{3.23}{3.23}$

3.23
0.00

3.7
3.74
3.74
0.00

$0.00 \quad 0.00$

$0.00 \quad 0.00$

49.4

$\frac{2.97}{2.13}$

49.4

$\frac{3.03}{2.16}$

49.4
.3 .20

0.84

0.00

0.870 .83

$\begin{array}{lllll}48.7 & 17.8 & 48.7 & 48.7 & 48.7 \\ \frac{2.80}{2.80} & \frac{2.81}{2.81} & \frac{2.81}{2.81} & \frac{2.70}{2.70} & \frac{2.70}{2.70} \\ 0.00 & 0.00 & 0.00 & 0.00 & 0.00\end{array}$

$\begin{array}{lll}0.00 & 0.00 & 0.00 \\ 0.00 & 0.00 & 0.00\end{array}$
48.7
2.70
2.70
0.00
0.00

$\begin{array}{llllllllllll}10.0 & 10.0 & 7.5 & 10.0 & 10.0 & 9.0 & 10.0 & 10.0 & 10.0 & 10.0 & 10.0 & 10.0 \\ \frac{2.32}{2.32} & \frac{2.32}{2.32} & \frac{2.32}{2.32} & \frac{2.41}{2.41} & \frac{2.41}{2.41} & \frac{2.41}{2.41} & \frac{2.41}{2.41} & \frac{2.41}{2.41} & \frac{2.41}{2.41} & \frac{2.41}{2.41} & \frac{2.41}{2.41} & \frac{2.41}{2.41} \\ 0.00 & 0.00 & 0.00 & 0.00 & 0.00 & 0.00 & 0.00 & 0.00 & 0.00 & 0.00 & 0.00 & 0.00 \\ 0.00 & 0.00 & 0.00 & 0.00 & 0.00 & 0.00 & 0.00 & 0.00 & 0.00 & 0.00 & 0.00 & 0.00\end{array}$

\begin{tabular}{r}
4.5 \\
4.47 \\
\hline 4.47 \\
0.00 \\
0.00
\end{tabular}

$\begin{array}{r}3.7 \\ 4.13 \\ \hline 4.13\end{array}$

4.13

0.00

$\begin{array}{r}3.7 \\ 4.54 \\ \hline 4.54\end{array}$

4.54
0.00

3.65
4.25

4.25

0.00

336,834

\begin{tabular}{l}
$3 Q 2000$ \\
TOTAL Mcf \\
\hline
\end{tabular}

YTD

TOTAL MCf

20

389

$1,230,000$

$3,018,258$

49.4
4.28

$\frac{4.28}{3.42}$

49.4
4.49

$\frac{4.49}{3.65}$

\begin{tabular}{l}
49.4 \\
3.81 \\
\hline
\end{tabular}

$\frac{3.81}{2.98}$

49.4
4.62

$\frac{4.62}{3.76}$

0.86

$\begin{array}{lll}0.84 & 0.83 & 0.86 \\ 0.00 & 0.00 & 0.00\end{array}$

$4,545,904$

$13,538,498$
.00

$\begin{array}{llllll}48.7 & 48.7 & 48.7 & 40.4 & 43.6 & 48.7 \\ \frac{3.10}{3.10} & \frac{3.39}{3.39} & \frac{4.59}{4.59} & \frac{4.54}{4.54} & \frac{4.01}{4.01} & \frac{4.80}{4.80} \\ 0.00 & 0.00 & 0.00 & 0.00 & 0.00 & 0.00 \\ 0.00 & 0.00 & 0.00 & 0.00 & 0.00 & 0.00\end{array}$

$4,064,597$

$12,921,056$

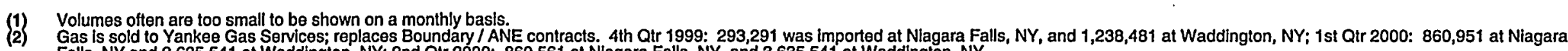

(3) Gas resold to Hopewell Cogeneratton Facility and other TEMCO customers. 


\section{VOLUME AND PRICE REPORT}

\section{Long-Term Imports}

Volumes (MMcf/d) \& Prices (\$/MMBTU) of Natural

Gas Imported During the Past 12 Months 10/01/99 - 09/30/00

Long-Term Importer

UNITED STATES GYPSUM COMPANY (Husky Oil Operations Ltd.)

AVG Daily Quantity

Total Price (Niagara Falls, NY)

Commodity Componen

Demand Component

Reservation Fee Component

UNITED STATES GYPSUM COMPANY

(Renaissance Energy, Ltd.)

AVG Daily Quantity

Total Price (Noyes, Minnesota)

Commodity Componen

Demand Component

Reservation Fee Component

UTILICORP UNITED, INC. (1)

(ProGas Limited)

AVG Daily Quantity

Total Price (Noyes, Minnesota)

Commodity Componen

Demand Component

Reservation Fee Component

UTILICORP UNITED, INC. (2)

(TCGS Ltd.)

AVG Daily Quantity

Total Price (Noyes, Minnesota)

Commodity Component

Demand Component

Reservation Fee Component

\section{VALLEY GAS COMPANY}

(Alberta NE Gas (TCGS Ltd. 1))

AVG Daily Quantity

Total Price (Waddington, NY)

Commodity Component

Demand Component

Reservation Fee Component

\begin{tabular}{|c|c|c|c|c|c|c|c|c|c|c|c|}
\hline $\begin{array}{l}\text { Auth. } \\
\text { Vols. } \\
\text { vols. }\end{array}$ & $\begin{array}{l}1999 \\
\text { Oct. }\end{array}$ & $\begin{array}{l}1999 \\
\text { Nov. }\end{array}$ & $\begin{array}{l}1999 \\
\text { Dec. }\end{array}$ & $\begin{array}{l}2000 \\
\text { Jan. }\end{array}$ & $\begin{array}{l}2000 \\
\text { Feb. }\end{array}$ & $\begin{array}{l}2000 \\
\text { Mar. }\end{array}$ & $\begin{array}{l}2000 \\
\text { Apr. }\end{array}$ & $\begin{array}{l}2000 \\
\text { May. }\end{array}$ & $\begin{array}{l}2000 \\
\text { Jun. }\end{array}$ & $\begin{array}{l}2000 \\
\underline{J u l} .\end{array}$ & $\begin{array}{l}2000 \\
\text { Aug. }\end{array}$ \\
\hline 13.6 & $\begin{array}{l}16.5 \\
3.08 \\
2.45 \\
0.63 \\
0.00\end{array}$ & $\begin{array}{l}16.6 \\
3.50 \\
2.87 \\
0.63 \\
0.00\end{array}$ & $\begin{array}{l}16.5 \\
\frac{3.01}{2.38} \\
0.63 \\
0.00\end{array}$ & $\begin{array}{l}16.5 \\
2.81 \\
2.18 \\
0.63 \\
0.00\end{array}$ & $\begin{array}{l}16.2 \\
2.90 \\
2.12 \\
0.78 \\
0.00\end{array}$ & $\begin{array}{l}16.5 \\
\frac{3.08}{2.30} \\
0.78 \\
0.00\end{array}$ & $\begin{array}{l}16.6 \\
3.38 \\
2.60 \\
0.78 \\
0.00\end{array}$ & $\begin{array}{l}16.5 \\
3.53 \\
2.75 \\
0.78 \\
0.00\end{array}$ & $\begin{array}{l}16.6 \\
4.04 \\
3.26 \\
0.78 \\
0.00\end{array}$ & $\begin{array}{l}16.5 \\
4.52 \\
3.74 \\
0.78 \\
0.00\end{array}$ & $\begin{array}{l}16.5 \\
4.08 \\
3.30 \\
0.78 \\
0.00\end{array}$ \\
\hline 13.5 & $\begin{array}{l}13.5 \\
2.70 \\
2.45 \\
0.25 \\
0.00\end{array}$ & $\begin{array}{l}13.5 \\
3.12 \\
2.87 \\
0.25 \\
0.00\end{array}$ & $\begin{array}{l}13.5 \\
2.63 \\
2.38 \\
0.25 \\
0.00\end{array}$ & $\begin{array}{l}13.5 \\
2.46 \\
2.18 \\
0.28 \\
0.00\end{array}$ & $\begin{array}{l}13.0 \\
2.40 \\
2.12 \\
0.28 \\
0.00\end{array}$ & $\begin{array}{l}13.5 \\
2.58 \\
2.30 \\
0.28 \\
0.00\end{array}$ & $\begin{array}{l}12.6 \\
\frac{2.88}{2.60} \\
0.28 \\
0.00\end{array}$ & $\begin{array}{l}12.2 \\
\frac{3.03}{2.75} \\
0.28 \\
0.00\end{array}$ & $\begin{array}{l}13.5 \\
3.54 \\
3.26 \\
0.28 \\
0.00\end{array}$ & $\begin{array}{l}13.5 \\
4.02 \\
3.74 \\
0.28 \\
0.00\end{array}$ & $\begin{array}{l}12.2 \\
3.58 \\
3.30 \\
0.28 \\
0.00\end{array}$ \\
\hline 2.7 & $\begin{array}{r}2.6 \\
2.67 \\
2.15 \\
0.50 \\
0.02\end{array}$ & $\begin{array}{r}2.6 \\
3.20 \\
2.66 \\
0.52 \\
0.02\end{array}$ & $\begin{array}{r}2.6 \\
2.27 \\
1.77 \\
0.48 \\
0.02\end{array}$ & $\begin{array}{r}2.6 \\
2.53 \\
1.98 \\
0.53 \\
0.02\end{array}$ & $\begin{array}{r}2.6 \\
2.84 \\
2.23 \\
0.59 \\
0.02\end{array}$ & $\begin{array}{r}2.6 \\
2.78 \\
2.21 \\
0.55 \\
0.02\end{array}$ & $\begin{array}{r}2.6 \\
3.10 \\
2.50 \\
0.58 \\
0.02\end{array}$ & $\begin{array}{r}2.4 \\
3.28 \\
2.68 \\
0.58 \\
0.02\end{array}$ & $\begin{array}{r}2.6 \\
4.57 \\
3.96 \\
0.59 \\
0.02\end{array}$ & $\begin{array}{r}2.6 \\
4.54 \\
3.94 \\
0.58 \\
0.02\end{array}$ & $\begin{array}{r}2.6 \\
4.00 \\
3.41 \\
0.57 \\
0.02\end{array}$ \\
\hline
\end{tabular}

7.2

$\begin{array}{rrrrrrrrrrrr}7.2 & 7.2 & 7.2 & 7.2 & 7.2 & 7.2 & 7.2 & 6.8 & 7.2 & 7.2 & 7.2 & 7.2 \\ \frac{2.68}{2.17} & \frac{3.20}{2.28} & \frac{2.27}{1.79} & \frac{2.55}{2.00} & \frac{2.83}{2.25} & \frac{2.79}{2.24} & \frac{3.11}{2.53} & \frac{3.29}{2.70} & \frac{4.61}{3.98} & \frac{4.58}{3.96} & \frac{4.04}{3.44} & \frac{4.88}{4.24} \\ 0.42 & 0.43 & 0.41 & 0.47 & 0.49 & 0.46 & 0.48 & 0.48 & 0.47 & 0.46 & 0.46 & 0.47 \\ 0.09 & 0.09 & 0.07 & 0.08 & 0.09 & 0.09 & 0.10 & 0.11 & 0.16 & 0.16 & 0.14 & 0.17\end{array}$

1.0
302000

TOTALMC

YTD

TOTAL MCf

$1,521,000 \quad 4,522,500$

$1,201,500$

$3,577,500$

236,526

702,863

663,045

$1,962,339$

87,000

268,000

(1) Volumes used by Michigan Gas Uilitites. 


\section{VOLUME AND PRICE REPORT}

\section{Long-Term Imports}

Volumes (MMct/d) \& Prices (\$/MMBTU) of Natural Gas Imported During the Past 12 Months 10/01/99 - 09/30/00

Long-Term Importer

VERMONT GAS SYSTEMS INC. (1) (TCGS Ltd.)

AVG Daily Quantity

Total Price (Highgate Spr., VT)

Commodity Componen

Demand Component

Reservation $\mathrm{Fee}$ Component

VERMONT GAS SYSTEMS, INC.

(Renaissance Energy Ltd.)

AVG Daily Quantity

Total Price (Highgate Spr., VT)

Commodity Componen

Reservation Fee Component

\section{VERMONT GAS SYSTEMS, INC. (2)}

(Various Suppliers

AVG Dally Quanithy

Total Price (Highgate Spr. VT)

Commodity Component

Demand Component

Reservation Fee Component

WISCONSIN ELECTRIC POWER CO. (GAS OPS)

(Progas Limited)

AVG Daily Quantity

Total Price (Noyes, Minnesota)

Commodity Component

Demand Component

Reservation Fee Component

Auth.
Vols.

Oct. Nov.

11.0

2000

2000

2000

2000

20002000

200020002000

$3 Q 2000$

TOTAL MCf

\section{YTD}

TOTAL MCf

8.0

19.0

\section{WISCONSIN ELECTRIC POWER CO. (GAS OPS)}

(TCGS Ltd.)

AVG Daily Quantity

Total Price (Noyes, Minnesota)

Commodity Component

Demand Componen

Reservation Fee Component
26.5

\begin{tabular}{rr}
8.7 & 9.1 \\
3.02 & 3.53 \\
\hline 1.88 & 2.39 \\
1.14 & 1.14 \\
0.00 & 0.00
\end{tabular}

11.1
2.67
1.77
0.90
0.00

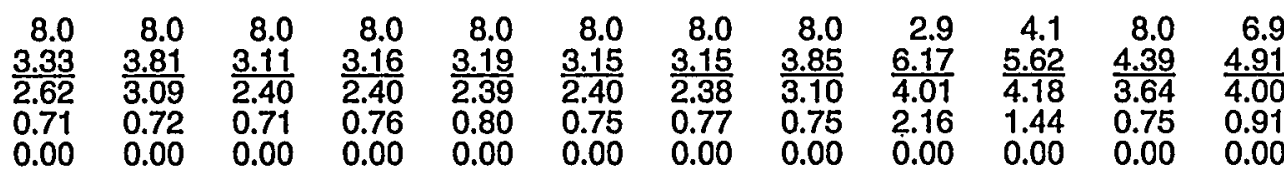

$\begin{array}{rrrrrrrrrrrr}1.0 & 2.1 & 8.7 & 13.4 & 12.0 & 6.2 & 4.0 & 0.0 & 0.0 & 0.0 & 0.0 & 0.0 \\ \frac{8.71}{2.25} & \frac{5.57}{2.29} & \frac{3.02}{2.24} & \frac{2.74}{2.23} & \frac{2.85}{2.24} & \frac{3.38}{2.26} & \frac{3.93}{2.17} & \frac{0.00}{0.00} & \frac{0.00}{0.00} & \frac{0.00}{0.00} & \frac{0.00}{0.00} & \frac{0.00}{0.00} \\ 6.46 & 3.28 & 0.78 & 0.51 & 0.61 & 1.12 & 1.76 & 0.00 & 0.00 & 0.00 & 0.00 & 0.00 \\ 0.00 & 0.00 & 0.00 & 0.00 & 0.00 & 0.00 & 0.00 & 0.00 & 0.00 & 0.00 & 0.00 & 0.00\end{array}$
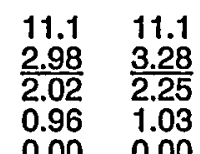

11.1
3.19

$\frac{3.19}{2.23}$

0.96

0.00

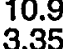

2.35

00

$\begin{array}{r}4.5 \\ 4.47 \\ \hline 2.18\end{array}$

7.0
4.83

0.9
4.03

2.18

1.54

$\begin{array}{rr}3.04 & 2.14 \\ 2.59 & 11.89\end{array}$

0.0011 .89

$\begin{array}{r}4.3 \\ 5.34 \\ \hline\end{array}$

$\frac{5.34}{2.82}$

2.52

.00

6.9

$\frac{4.91}{4.00}$

0.91
0.00

$0.00 \quad 0.00$

0.00

0.00

00 $\begin{array}{r}4.1 \\ 5.63 \\ \hline 3.04\end{array}$ \begin{tabular}{lr}
10.7 & 4.3 \\
2.67 & 3.96 \\
\hline 2.15 & 2.66 \\
0.46 & 1.20 \\
0.06 & 0.10
\end{tabular}

\begin{tabular}{ll}
10.7 & 11.0 \\
2.26 & 2.54 \\
\hline 1.77 & 1.98 \\
0.46 & 0.52 \\
0.03 & 0.04
\end{tabular}

11.0
2.82
2.23
0.55
0.04

10.3
2.81
2.21
0.55
0.05

\begin{tabular}{l}
10.7 \\
3.08 \\
\hline 2.50 \\
0.53 \\
0.05
\end{tabular}

$\begin{array}{r}10.2 \\ 3.27 \\ \hline\end{array}$

$\frac{3.27}{2.68}$

0.06

0.05

\begin{tabular}{l}
10.7 \\
4.57 \\
\hline 3.96 \\
0.53
\end{tabular}

0.53
0.08

\begin{tabular}{l}
10.7 \\
4.53 \\
\hline 3.94 \\
0.5 \\
0.08
\end{tabular}

10.7

$\frac{3.99}{3.41}$

$\begin{array}{lll} & 0.51-0.53\end{array}$

$\begin{array}{lll}0.08 & 0.07 \quad 0.08\end{array}$

$\begin{array}{llll}26.6 & 12.4 & 26.6 & 26.3 \\ 2.61 & 3.68 & 2.26 & 2.48 \\ \frac{3.12}{2.56} & \frac{1.79}{1.97} \\ 0.43 & 0.96 & 0.42 & 0.45\end{array}$

0.06

\begin{tabular}{l}
23.0 \\
2.81 \\
\hline 2.19 \\
0.55
\end{tabular}

2.19
0.55

0.55
0.07 \begin{tabular}{l}
24.2 \\
3.05 \\
\hline 2.43 \\
0.54 \\
0.08
\end{tabular} \begin{tabular}{l}
25.0 \\
3.16 \\
\hline 2.58 \\
0.50
\end{tabular}

0.50
0.08

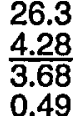

0.49
0.11 \begin{tabular}{l}
26.3 \\
4.24 \\
\hline 3.67 \\
0.46
\end{tabular}

0.46
0.11

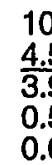

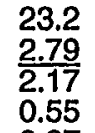

$\frac{2.79}{2.17}$

0.55
0.07
281,094

$1,969,948$

582,021

$1,884,301$

0

$1,075,282$

(1) Daily contract demand was reduced from $32 \mathrm{MMcf}$ to 21 MMcf, effective April 1, 1995. On November 1, 1998, the dally contract demand was reduced to 11 MMct.
(2) This import is part of a gas services agreement with Gaz Metropolltain Limited Partnership. 


\section{VOLUME AND PRICE REPORT}

\section{Long-Term Imports}

Volumes (MMcf/d) \& Prices (\$/MMBTU) of Natural

Gas Imported During the Past 12 Months 10/01/99 - 09/30/00

Long-Term Importer

WISCONSIN FUEL \& LIGHT COMPANY (Progas Limited)

AVG Daily Quantity

Total Price (Noyes, Minnesota)

Commodity Component

Demand Component

Reservation Fee Component

WISCONSIN FUEL \& LIGHT COMPANY

(TCGS Ltd.)

AVG Daily Quantity

Total Price (Noyes, Minnesota)

Commodity Component

Demand Component

Reservation Fee Component

WISCONSIN GAS COMPANY (1)

(ProGas Limited (I))

AVG Daily Quantity

Total Price (Noyes, Minnesota)

Commodity Component

Demand Component

Reservation Fee Component

WISCONSIN GAS COMPANY

(ProGas Limited (II))

AVG Daily Quantity

Total Price (Noyes, Minnesota)

Commodity Componen

Demand Component

Reservation Fee Component

WISCONSIN GAS COMPANY (2)

(TCGS Ltd.)

AVG Daily Quantity

Total Price (Noyes, Minnesota)

Commodity Component

Demand Component

Reservation Fee Component
Vols
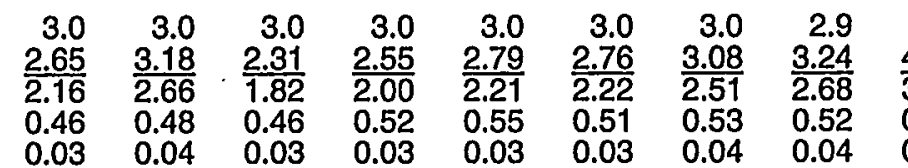

\begin{tabular}{r}
3.0 \\
4.48 \\
\hline 3.89 \\
0.53 \\
0.06
\end{tabular}

\begin{tabular}{r}
3.0 \\
4.49 \\
\hline 3.92 \\
0.51 \\
0.06
\end{tabular}

3.0
3.96
3.40

0.51

0.05

7.4

\begin{tabular}{rrr}
7.4 & 3.7 & 7.3 \\
2.66 & 3.58 & 2.35 \\
\hline 2.18 & $\frac{3.55}{2.55}$ & 1.88 \\
0.42 & 0.87 & 0.41
\end{tabular}

0.42

0.06

0.16

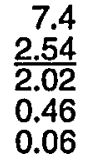

7.2
2.78
2.21
0.50

6.9
$\frac{3.21}{2.65}$
0.48

6.6

\begin{tabular}{rr}
2.2 & 6.2 \\
4.21 & 2.01 \\
\hline 2.66 & 1.45 \\
1.44 & 0.50 \\
0.11 & 0.06
\end{tabular}

\begin{tabular}{rr}
6.7 & 6.7 \\
2.27 & $\frac{2.54}{1.94}$ \\
\hline 1.77 & 0.58 \\
0.46 & 0.52 \\
0.04 & 0.04
\end{tabular}

6.7
2.82
2.23
0.55
0.04

0.110 .06

28.5

\begin{tabular}{rrrr}
8.4 & 26.9 & 28.9 & 28 \\
4.39 & 2.01 & 2.27 & 2.53 \\
\hline 2.66 & 1.45 & $\frac{2.77}{1.9}$ \\
1.60 & 0.50 & 0.46 & 0.51 \\
0.13 & 0.06 & 0.04 & 0.04
\end{tabular}

89.4

$\begin{array}{lllll}68.5 & 19.4 & 86.7 & 84.1 & 87.8 \\ \frac{2.61}{2.12} & \frac{4.41}{2.56} & \frac{2.28}{1.79} & \frac{2.54}{1.97} & \frac{2.78}{2.19} \\ 0.43 & 1.57 & 0.44 & 0.51 & 0.52 \\ 0.06 & 0.28 & 0.05 & 0.06 & 0.07\end{array}$

.7 .4
$\frac{2.74}{2.22}$
0.45
0.07

7.3
3.04
2.49

2.49

0.48
0.07

0.48
0.08

\begin{tabular}{r}
7.3 \\
4.30 \\
\hline 3.71 \\
0.48 \\
0.11
\end{tabular}

4.5
4.68
3.75

$\frac{4.68}{3.75}$

0.75

0.75
0.18

6.7
$\frac{2.77}{2.21}$
0.52
0.04

\begin{tabular}{r}
6.6 \\
3.09 \\
\hline 2.50 \\
0.54
\end{tabular}

$\begin{array}{r}6.4 \\ 3.27 \\ \hline .68\end{array}$

2.68

$\begin{array}{r}6.4 \\ 4.59 \\ \hline 3.96\end{array}$

0.05

0.06

0.55

$\begin{array}{r}2.3 \\ 5.62 \\ \hline .94\end{array}$

$\frac{5.62}{3.94}$

1.46
0.22

$\frac{4.00}{3.41}$

3.41

0.52

$\begin{array}{ll}0.07 & 0.09\end{array}$

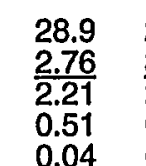

28.7
$\frac{3.09}{2.50}$
0.54
0.05

27.1
.3 .28
2.68
0.54
0.06

27.7
4.59
3.96
0.55
0.08

10.2
5.61

$\frac{5.61}{3.94}$

1.45

\begin{tabular}{l}
28.7 \\
3.99 \\
\hline 3.41
\end{tabular}

3.41

0.51
0.07

\begin{tabular}{l}
28.7 \\
4.82 \\
\hline 4.21 \\
0.53 \\
0.08
\end{tabular}

$2,066,273$

587,430

$1,913,328$

475,123

$1,667,469$

$\begin{array}{lll}75.5 & 89.3 & 86.3 \\ \frac{2.81}{2.17} & \frac{2.98}{2.43} & \frac{3.13}{2.58} \\ 0.56 & 0.49 & 0.49 \\ 0.08 & 0.06 & 0.06\end{array}$

0.56

0.06
89.3
4.28
3.68
0.49
0.11

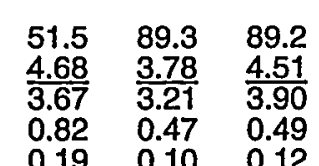

(1) Imports include special spot purchases from ProGas.
Imports include special spot purchases from TransCanada. 


\section{VOLUME AND PRICE REPORT \\ Long-Term Imports}

Volumes (MMcf/d) \& Prices (\$MMBTU) of Natural

Gas Imported During the Past 12 Months 10/01/99 - 09/30/00

\section{Long-Term Importer}

\section{WISCONSIN GAS COMPANY (1)}

(TCGS Ltd.)

AVG Daily Quantity

Total Price (Noyes, Minnesota)

Commodity Component

Demand Component

Reservation Fee Component

WISCONSIN POWER \& LIGHT COMPANY

(ProGas Limited)

AVG Daily Quantity

Total Price (Noyes, Minnesota)

Commodity Componen

Demand Component

Reservation Fe日 Component

\section{WISCONSIN POWER \& LIGHT COMPANY}

(TCGS Ltd.)

AVG Daily Quantity

Total Price (Noyes, Minnesota)

Commodity Componen

Reservation Fee Component

Auth.

Vo

Vols.

\begin{tabular}{lr}
20.9 & 6.3 \\
2.60 & 4.26 \\
\hline 2.12 & 2.56 \\
0.42 & 1.45
\end{tabular}

$\begin{array}{ll}0.06 & 0.25\end{array}$

3.4

\begin{tabular}{rr}
3.4 & 3.4 \\
2.65 & 3.18 \\
\hline 2.15 & 2.66 \\
0.50 & 0.52 \\
0.00 & 0.00
\end{tabular}

8.4

\begin{tabular}{rr}
8.3 & 2.8 \\
$\frac{2.62}{2}$ & $\frac{4.14}{2.56}$ \\
\hline 2.50 & 1.58 \\
0.50 & 0.00
\end{tabular}

\begin{tabular}{r}
8.3 \\
2.27 \\
\hline 1.79 \\
0.48 \\
0.00
\end{tabular}

8.3
2.51
1.97
0.54
0.00

\begin{tabular}{r}
8.3 \\
2.76 \\
\hline 2.19 \\
0.57 \\
0.00
\end{tabular}

\begin{tabular}{r}
8.3 \\
2.72 \\
\hline 2.17 \\
0.55 \\
0.00
\end{tabular}

8.3
$\frac{3.00}{2.43}$
0.57
0.00

\begin{tabular}{r}
8.3 \\
3.13 \\
\hline 2.58 \\
0.55 \\
0.00
\end{tabular}

\begin{tabular}{r}
8.3 \\
4.28 \\
\hline 3.68 \\
0.60 \\
0.00
\end{tabular}

7.3
3.67
0.69

$\frac{8.7}{3.21}$

WISCONSIN PUBLIC SERVICE CORPORATION

(ProGas Limited)

AVG Daily Quantity

Total Price (Noyes, Minnesota)

11.1

\begin{tabular}{llll}
11.1 & 11.1 & 11.1 & 11.1 \\
2.69 & 3.18 & 2.26 & 2.53 \\
\hline 2.15 & $\frac{3.66}{2.66}$ & $\frac{2.77}{1.98}$ \\
0.46 & 0.48 & 0.46 & 0.52
\end{tabular}

11.1
2.81
2.23
0.55

\begin{tabular}{l}
11.1 \\
2.75 \\
\hline 2.21 \\
0.51 \\
0.03
\end{tabular}

11.1
3.07
2.50
0.53
0.04

\begin{tabular}{l}
10.4 \\
3.26 \\
\hline 2.68 \\
0.54 \\
0.04
\end{tabular}

11.1
4.55
3.96
0.53
0.06

11.1
4.51
3.94

3.94

0.51
0.06

$\begin{array}{ll}11.1 & 11.1\end{array}$

$\frac{3.97}{3.41} \quad \frac{4.80}{4.21}$

$\begin{array}{ll}3.41 & 4.21 \\ 0.51 & 0.53\end{array}$

Reservation Fee Component

\section{WISCONSIN PUBLIC SERVICE CORPORATION}

(TCGS Litd.)

AVG Daily Quantity

Total Price (Noyes, Minnesota)

27.4

Commodity Componen

Demand Component

Reservation Fee Component

$\begin{array}{llll}27.6 & 17.3 & 27.5 & 27.5 \\ \frac{2.61}{2.12} & \frac{3.39}{2.56} & \frac{2.26}{1.79} & \frac{2.51}{1.97} \\ 0.43 & 0.71 & 0.42 & 0.48 \\ 0.06 & 0.12 & 0.05 & 0.06\end{array}$

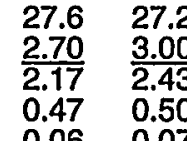

$\begin{array}{ll}0.06 & 0.07\end{array}$

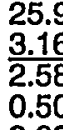

\begin{tabular}{l}
25.9 \\
3.16 \\
\hline 2.58 \\
0.50 \\
0.08
\end{tabular}

\begin{tabular}{l}
27.2 \\
4.28 \\
\hline 3.68 \\
0.49 \\
0.11
\end{tabular}

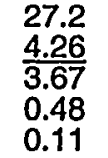

27.7
3.7
3.2
0.47
0.10
3Q 2000

TOTAL MCI

133,618

484,302

310,533

924,773

727,942

$2,245,811$

$1,019,090$

$3,014,511$

(1) Imports include special spot purchases from TransCanada. 
VOLUME AND PRICE REPORT

\section{Long-Term Imports}

Volumes (MMcf/d) \& Prices (\$/MMBTU) of Natural Gas Imported During the Past 12 Months 10/01/99 - 09/30/00

\section{Long-Term Importer}

\section{Auth}

YANKEE GAS SERVICES CO.

(Alberta NE Gas (AEC Oil \& Gas Company))

AVG Daily Quantity

Total Price (Waddington, NY)

Commodity Component

Demand Component

Reservation Fee Component

YANKEE GAS SERVICES CO. (1)

(Alberta NE Gas (TCGS Ltd. 1))

AVG Daily Quantity

Total Price (Waddington, NY)

Commodity Component

Demand Component

Reservation Fee Component

YANKEE GAS SERVICES CO. (2)

(Alberta NE Gas (TCGS Ltd. 2))

AVG Daily Quantity

Total Price (Waddington, NY)

Commodity Component

Demand Component

Reservation Fee Component

\section{YANKEE GAS SERVICES CO.}

(Alberta Northeast Gas (ProGas))

AVG Daily Quantity

Total Price (Waddington, NY)

Commodity Component

Demand Component

Reservation Fee Component

YANKEE GAS SERVICES CO.

(Alberta Northeast Gas (Producers Marketing))

AVG Daily Quantity

Total Price (Waddington, NY)

Commodity Componen

Demand Component

Reservation Fee Component

Auth.

$19991999 \quad 1999 \quad 2000$

Oct.

3.9

\begin{tabular}{rrr}
3.9 & 3.9 & 3.9 \\
2.94 & 3.34 & 2.67 \\
\hline 2.06 & 2.46 & 1.79 \\
0.88 & 0.88 & 0.88 \\
0.00 & 0.00 & 0.00
\end{tabular}

28.7

\begin{tabular}{rrr}
28.7 & 28.7 & 0.0 \\
2.73 & $\frac{3.18}{2.39}$ & 0.00 \\
\hline 1.97 & 0.00 \\
0.76 & 0.79 & 0.00 \\
0.00 & 0.00 & 0.00
\end{tabular}

Jan.

2000
Feb.

2000

2000

2000

3.9
2.97
2.03
0.94
0.00

3.9
$\frac{3.32}{2.38}$
0.94
0.00

0.0
0.00

$\frac{0.00}{0.00}$

0.0
0.00

$\frac{0.00}{0.00}$

$0.00 \quad 0.00$

\begin{tabular}{r}
3.9 \\
3.57 \\
\hline 2.65 \\
0.92 \\
0.00
\end{tabular}

$\begin{array}{ll}28.7 & 28.7 \\ & 2.73 \\ & 1.97 \\ & 0.76 \\ \cdot & 0.00\end{array}$

$0.00 \quad 0.00$

0.0
0.00
0.00
0.00
0.00

$\begin{array}{r}0.0 \\ 0.00 \\ \hline .00\end{array}$

$\frac{0.00}{0.00}$

0.00

0.00
0.00

0.0
0.00
0.00
0.00
0.00

0.0
0.00
0.00
0.00

0.0
0.00

$\frac{0.00}{0.00}$

0.00
0.00

$0.00 \quad 0.00$

0.0
0.00
0.00
0.00
0.00

0.0
0.00
0.00
0.00

0.0
0.00
0.00

$\frac{0.00}{0.00}$

0.0
0.00
0.00
0.00

0.00

9.9

\begin{tabular}{rrrr}
9.9 & 5.2 & 9.9 & 9.9 \\
2.99 & $\frac{3.38}{2.48}$ & $\frac{2.79}{1.87}$ & $\frac{2.98}{1.96}$ \\
\hline 2.07 & 2.48 & 0.92 & 1.02 \\
0.92 & 0.90 & 02 \\
0.00 & 0.00 & 0.00 & 0.00
\end{tabular}

9.9
3.42
2.40
1.02
0.00

9.9
3.36
2.35
1.01
0.00

\begin{tabular}{r}
9.9 \\
3.49 \\
\hline 2.47 \\
1.02 \\
0.00
\end{tabular}

9.9
$-\frac{3.61}{2.61}$
1.00

0.00

$\begin{array}{r}0.0 \\ 0.00 \\ \hline 0.00\end{array}$

0.00
0.00

0.00

5.2

$\begin{array}{rrrr}5.2 & 5.2 & 5.2 & 5.2 \\ \frac{2.99}{2.10} & \frac{3.37}{2.44} & \frac{2.79}{1.90} & \frac{2.98}{1.99} \\ 0.89 & 0.93 & 0.89 & 0.99 \\ 0.00 & 0.00 & 0.00 & 0.00\end{array}$

\begin{tabular}{r}
5.2 \\
3.42 \\
\hline 2.44 \\
0.98 \\
0.00
\end{tabular}

\begin{tabular}{r}
5.2 \\
3.37 \\
\hline 2.39 \\
0.98 \\
0.00
\end{tabular}

5.2
$\frac{3.49}{2.51}$
0.98
0.00

5.2
$\frac{3.60}{2.64}$
0.96
0.00

2000

2000

3.9
4.72
3.79
0.93
0.00

$\begin{array}{r}3.9 \\ 4.71 \\ \hline 3.78\end{array}$

$\frac{4.71}{3.78}$

0.93

$\frac{4.33}{3.41}$

3.41

3.9
4.92

4.00

0.92

0.0

$\frac{0.00}{0.00}$

0.0

$\frac{0.00}{0.00}$

0.0
0.00
0.00
0.00
0.00

0.00
0.00

0.0

$\frac{0.00}{0.00}$

0.00

0.00
0.00

0.0

$\frac{0.00}{0.00} \quad \frac{0.00}{0.00}$

0.00

0.0
.00
0.00
.00

0

0.00

.

0.0

$\frac{0.00}{0.00}$

0.00
0.00

$\begin{array}{r}0.0 \\ 0.00 \\ \hline 0.00\end{array}$

$\frac{0.00}{0.00}$

0.00

0.00
0.00

9.9

$\frac{4.70}{3.69}$

9.9

$\frac{4.76}{3.75}$

$\begin{array}{r}9.9 \\ 4.33 \\ \hline 3.33\end{array}$

$\frac{4.33}{3.33}$

9.9

$\frac{5.05}{4.05}$

$\begin{array}{llll}1.01 & 1.01 & 1.00 & 1.00 \\ 0.00 & 0.00 & 0.00 & 0.00\end{array}$

(1) Beginning on 12/1/99, these volumes will be imported and reported under TransCanada Gas Services' long-term authorization in Order Number 1543.
(2) Beginning on 12/1/99, these volumes will be Imported and reported under TransCanada Gas Services' long-term authorization in Order Number 1543 . 
VOLUME AND PRICE REPORT

Long-Term Imports

Volumes (MMcf/d) \& Prices (\$MMBTU) of Natural

Gas Imported During the Past 12 Months 10/01/99 - 09/30/00

Long-Term Importer

$\begin{array}{lllllllllllll}\text { Auth. } & 1999 & 1999 & 1999 & 2000 & 2000 & 2000 & 2000 & 2000 & 2000 & 2000 & 2000 & 2000\end{array}$

vols. Oct. Nov. Dec. Jan. Feb. Mar. Apr. May. Jun. Jul. Aug. Sep.

3Q 2000

TOTAL MC

YTD

Nov Dec. Jan. Feb. Mar.

Total Mcf

$364,103,020$

$1,095,212,43$ 


\section{VOLUME AND PRICE REPORT}

\section{Long Term Exports}

Volumes (MMcf/d) \& Prices (\$MMBTU) of Natural

Gas Exported During the Past 12 Months 10/01/99 - 09/30/00

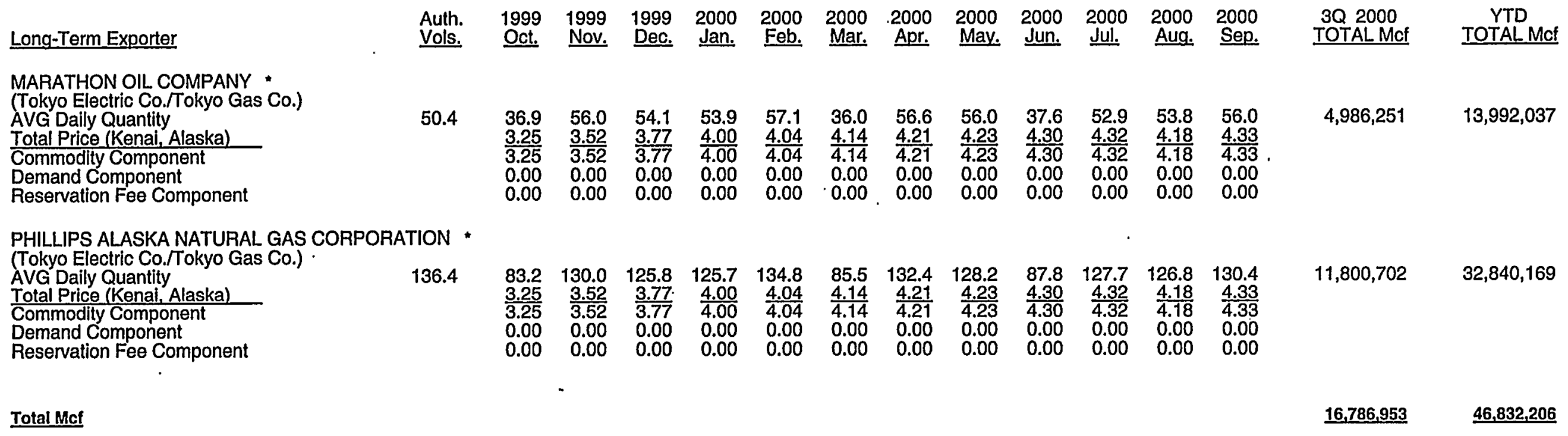




\section{SHORT-TERM BLANKET IMPORTS}

Year \&

Month

Active

Importers
Est. Volumes

(MMCF)
Weighted Avg.

Price (\$/MMBTU)

1998

January

February

March

April

May

June

July

August

September

October

November

December

1999

January

February

March

April

May

June

July

August

September

October

November

December

2000

$\begin{array}{ll}\text { January } & 83 \\ \text { February } & 80 \\ \text { March } & 85 \\ \text { April } & 81 \\ \text { May } & 79 \\ \text { June } & 82 \\ \text { July } & 80 \\ \text { August } & 82 \\ \text { September } & 79\end{array}$

$\begin{array}{ll}156808 & 1.81 \\ 132208 & 1.66 \\ 138012 & 1.72 \\ 135700 & 1.79 \\ 133217 & 1.74 \\ 128632 & 1.63 \\ 151492 & 1.72 \\ 163301 & 1.63 \\ 152042 & 1.51 \\ 149495 & 1.78 \\ 138962 & 1.99 \\ 153651 & - \\ \end{array}$

178429

1.89

167243

171699

156277

173298

160951

173677

190654

185057

182480

195288

193581

1.65

1.71

2.05

2.01

2.08

2.29

2.53

2.41

2.73

2.25

$194466 \quad 2.33$

$176979 \quad 2.45$

$184686 \quad 2.53$

$176596 \quad 2.78$

165565

$180577 \quad 3.85$

$199771 \quad 3.87$

$199230 \quad 3.57$

$188258 \quad 4.09$ 
Page -56

\section{SHORT-TERM IMPORTERS}

Estimated Volumes (MMCF)

AEC MARKETING (USA) INC.

AEC WEST LTD.

ALCOA, INC.

ALLIANCE PIPELINE L.P.

AMOCO CANADA MARKETING CORPORATION

AMOCO ENERGY TRADIN CORP. (LNG)

ANDROSCOGGIN ENERGY LLC

AQUILA ENERGY MARKETING CORPORATION

AROCO PRODUCTS COMPANY

AVISTA CORPORATION

AVISTA ENERGY,INC.

BARRINGTON PETROLEUM LTD.

BC GAS UTUTYY LTD.

BIG SKY GAS MARKETING

BURLINGTON RESOURCES TRADING INC.

CABOT ENERGY SERVICE CORPORATION

CABOT OIL \& GAS MARKETING CORPORATION

CANWEST GAS SUPPLY U.S.A., INC.

CARTHAGE ENERGY SERVICES, INC.

CASCADE NATURAL GAS CORPORATION

CHEVRON U.S.A. INC.

CMS MARKETING SERVICES AND TRADING COMPANY (1)

COAST ENERGY GROUP

COENERGY TRADING COMPANY

COMMONWEALTH GAS COMPANY

CORAL ENERGY RESOURCES, LP.

CORAL ENERGY RESOURCES, L.P. (LNG)

CRESTAR ENERGY MARKETING CORPORATION

CXY ENERGY MARKETING (U.S.A.) INC.

DARTMOUTH POWER ASSOCIATES L.P.

DEK ENERGY COMPANY

DISTRIGAS CORPORATION (2)

DUKE ENERGY LNG MKTG \& MGMT CO (3)

DUKE ENERGY LNG SALES, INC. (4)

DUKE ENERGY TRADING AND MKTG SERVICES LLC.

DYNEGY MARKETING \& TRADE

EL PASO ENERGYMARKETING COMPANY

ENERGY USA-TPC CORP

ENERGY WEST RESOURCES, INC.

ENGAGE ENERGY CANADA, L.P.

ENGAGE ENERGY U.S., L.P.

ENRON ENERGY SERVICES, INC.

ENRON INTERNATIONAL GAS SALES COMPANY

ENRON NORTH AMERICA CORPORATION

H.Q. ENERGY SERVICES (U.S.) INC.

HESS ENERGYINC.

HESS ENERGY SERVICES COMPANY, LLC

\begin{tabular}{|c|c|c|c|c|}
\hline $\begin{array}{r}1999 \\
\text { Jul-Sep. } \\
\end{array}$ & $\begin{array}{c}1999 \\
\text { Oct.-Dec. }\end{array}$ & $\begin{array}{c}2000 \\
\text { Jan.-Mar. }\end{array}$ & $\begin{array}{r}2000 \\
\text { Apr.-Jun. }\end{array}$ & $\begin{array}{r}2000 \\
\text { Jul.-Sep. }\end{array}$ \\
\hline 6581 & 6346 & 6278 & 6312 & 7219 \\
\hline 7406 & 7378 & 7524 & 6992 & 6686 \\
\hline 1286 & 1516 & 1768 & 1506 & 1414 \\
\hline 0 & 0 & 0 & 131 & 4620 \\
\hline 10099 & 13086 & 16422 & 13836 & 17423 \\
\hline 0 & 0 & 0 & 0 & 1805 \\
\hline 0 & 167 & 289 & 85 & 61 \\
\hline 5340 & 10701 & 3402 & 4324 & 5194 \\
\hline 2351 & 2335 & 2261 & 1566 & 1633 \\
\hline 3365 & 6137 & 5210 & 3398 & 3161 \\
\hline 11407 & 12689 & 8171 & 7317 & 6170 \\
\hline 147 & 451 & 446 & 440 & 449 \\
\hline 127 & 896 & 211 & 277 & 218 \\
\hline 762 & 991 & 872 & 858 & 764 \\
\hline 0 & 0 & $\cdot \quad 0$ & 0 & 7070 \\
\hline 0 & 0 & 0 & 2607 & 0 \\
\hline 538 & 507 & 1 & 0 & 0 \\
\hline 1406 & 2028 & 1659 & 854 & 438 \\
\hline 1549 & 1462 & 737 & 737 & 0 \\
\hline 40 & 3596 & 1847 & 694 & 4348 \\
\hline 144 & 127 & 79 & 152 & 138 \\
\hline 6864 & 6949 & 3069 & 19620 & 25635 \\
\hline 1752 & 2309 & 1254 & 885 & 1927 \\
\hline 177 & 171 & 1250 & 987 & 241 \\
\hline 0 & 276 & 414 & 402 & 408 \\
\hline 5121 & 6456 & 9052 & 9578 & 9221 \\
\hline 2576 & 0 & 0 & 0 & 10093 \\
\hline 306 & 142 & 0 & 25 & 63 \\
\hline 14051 & 15079 & 17086 & 12111 & 11884 \\
\hline 302 & 384 & 100 & 121 & 207 \\
\hline 1056 & 1062 & 1049 & 1046 & 1059 \\
\hline 5860 & 5874 & 4433 & 0 & 0 \\
\hline 5676 & 0 & 0 & 0 & 0 \\
\hline 48 & 1651 & 966 & 0 & 0 \\
\hline 49336 & 61024 & 80555 & 78649 & 86600 \\
\hline 5981 & 1982 & 4208 & 5887 & 5515 \\
\hline 643 & 1392 & 3606 & 1774 & 2248 \\
\hline 0 & 0 & 0 & 1614 & 2905 \\
\hline 508 & 446 & 0 & 0 & 0 \\
\hline 6792 & 6675 & 7541 & 4357 & 3889 \\
\hline 18792 & 22861 & 19701 & 14931 & 17218 \\
\hline 0 & 904 & 1341 & 2400 & 3322 \\
\hline 0 & 2713 & 0 & 0 & 2740 \\
\hline 27654 & 24844 & 15762 & 18402 & 17288 \\
\hline 1380 & 1380 & 1365 & 1380 & 1380 \\
\hline 13313 & 6640 & 2315 & 121 & 308 \\
\hline 3724 & 3742 & 3568 & 3691 & 3713 \\
\hline
\end{tabular}

(1) Sales for resale rather than imports. Actual imports: 3rd Qtr 99 (9,677); 4th Qtr $99(9,496) ; 1$ st Qtr $00(3,069) ; 2$ nd Qtr 00 (19,620); 3rd Qtr 00 (25,635).

(2) Actual imports: 3rd Qtr $99(5,860)$; 4th Qtr $99(5,874)$; 1st Qtr $00(4,433)$; 2nd Qtr $00(0)$; 3rd Qtr $00(0)$.

(3) Actual imports: 3rd Qtr 99 (5,162); 4th Qtr $99(0)$; 1st Qtr $00(0)$; 2nd Qtr $00(0)$; 3rd Qtr $00(0)$.

(4) Actual imports: 3rd Qtr 99 (456); 4th Qtr 99 (2,671); 1st Qtr 00 (498); 2nd Qtr 00 (945); 3rd Qtr 00 (703). 


\section{SHORT-TERM IMPORTERS \\ Estimated Volumes (MMCF)}

HUSKY GAS MARKETING, INC.

IDACORP, ENERGY SOLUTIONS L.P. (1)

IGI RESOURCES, INC.

INDECK-OSWEGO, L.P.

INDECK-YERKES, L.P.

INTERENERGY CORPORATION

MONTANA-DAKOTA UTILITIES CO.

MURPHY GAS GATHERING

NATIONAL FUEL GAS DISTRIBUTION

NATIONAL FUEL RESOURCES, INC.

NEW YORK STATE ELECTRIC \& GAS CORPORATION

NORTH AMERICAN ENERGY, INC.

NORTHEAST GAS MARKETS L.L.C.

NORTHERN STATES POWER COMPANY (WI)

NOATHWEST NATURAL GAS COMPANY

NUMAC ENERGY (U.S.) INC.

ONYX GAS MARKETING COMPANY, L.C.

PAN-ALBERTA GAS (U.S.), INC.

PANCANADIAN PETROLEUM COMPANY

PAWTUCKET POWER ASSOCIATES L.P.

PEMEX GAS

PETRO-CANADA HYDROCARBONS, INC.

PETROCOM ENERGY GROUP, LTD.

PG\&E CORE PROCUREMENT DEPT.

POCO MARKETING LTD.

PORTLAND GENERAL ELECTRIC COMPANY

PORTLAND NATURAL GAS TRANSMISSION SYSTEM

PRAIRIELANDS ENERGY MARKETING, INC.

PREMSTAR ENERGY CANADA, LTD.

PROGAS U.S.A., INC.

PUGET SOUND ENERGY, INC.

QUESTAR ENERGY TRADING COMPANY

RELIANT ENERGY SERVICES, INC.

RENAISSANCE ENERGY (U.S.), INC.

ROCHESTER GAS \& ELECTRIC CORPORATION

SACRAMENTO MUNICIPAL UTILITY DISTRICT

SAN DIEGO GAS \& ELECTRIC

SEMPRA ENERGY TRADING CORPORATION

SIERRA PACIFIC POWER COMPANY

SOUTHERN COMPANY ENERGY MARKETING L.P.

SPRAGUE ENERGY CORPORATION

ST. LAWRENCE GAS COMPANY, INC.

STAMPEDER ENERGY (U.S.) INC.

SUNCOR ENERGY INC.

SUNOMA ENERGY CORPORATION

TENASKA MARKETING VENTURES

TEXACO ENERGY MARKETING L.P.

\begin{tabular}{|c|c|c|c|c|}
\hline $\begin{array}{r}1999 \\
\text { Jul.-Sep. }\end{array}$ & $\begin{array}{c}1999 \\
\text { Oct.-Dec. }\end{array}$ & $\begin{array}{c}2000 \\
\text { Jan.-Mar. }\end{array}$ & $\begin{array}{c}2000 \\
\text { Apr.-Jun. }\end{array}$ & $\begin{array}{r}2000 \\
\text { Jul.-Sep. }\end{array}$ \\
\hline 10795 & 8457 & 9389 & 8332 & 9104 \\
\hline 0 & 610 & 891 & 0 & 0 \\
\hline 17589 & 16576 & 14369 & 14990 & 15057 \\
\hline 1103 & 1053 & 1096 & 1096 & 1108 \\
\hline 1106 & 1108 & 1096 & 1096 & 1108 \\
\hline 858 & 289 & 0 & 0 & 0 \\
\hline 925 & 941 & 873 & 849 & 845 \\
\hline 299 & 299 & 296 & 296 & 299 \\
\hline 1639 & 1248 & 1660 & 4053 & 1637 \\
\hline 3361 & 3861 & 3791 & 4114 & 2968 \\
\hline 0 & 5 & 0 & 0 & 0 \\
\hline 166 & 234 & 258 & 324 & 518 \\
\hline 0 & 2702 & 961 & 353 & 0 \\
\hline 0 & 663 & 988 & 0 & 0 \\
\hline 1193 & 2818 & - 5124 & 1715 & 360 \\
\hline 2841 & 2238 & 1900 & 1894 & 1904 \\
\hline 1756 & 0 & 0 & 0 & 0 \\
\hline 1001 & 883 & 934 & 776 & 597 \\
\hline 22994 & 24551 & 25792 & 24793 & 25451 \\
\hline 135 & 55 & 91 & 41 & 0 \\
\hline 12614 & 13928 & 3956 & 756 & 61 \\
\hline 13918 & 13941 & 13828 & 13787 & 13975 \\
\hline 0 & 0 & 802 & 2751 & 1197 \\
\hline 52397 & 53204 & 52899 & 52702 & 56709 \\
\hline 17617 & 16469 & 16299 & 16234 & 9452 \\
\hline 4891 & 4611 & 4683 & 4398 & 4757 \\
\hline 0 & 0 & 0 & 52 & 0 \\
\hline 0 & 0 & 0 & 0 & 55 \\
\hline 1676 & 378 & 0 & 215 & 0 \\
\hline 8089 & 8864 & 9184 & 5182 & 5740 \\
\hline 2061 & 3180 & 5484 & 3714 & 9817 \\
\hline 15 & 349 & 761 & 0 & 0 \\
\hline 1423 & 4056 & 3051 & 2857 & 2888 \\
\hline 13469 & 10061 & 8853 & 9343 & 13179 \\
\hline 2514 & 3004 & 6194 & 3494 & 2526 \\
\hline 528 & 0 & 0 & 1164 & 1140 \\
\hline 2033 & 1882 & 1883 & 2179 & 1332 \\
\hline 4460 & 6657 & 12808 & 14944 & 17928 \\
\hline 2504 & 3176 & 2930 & 3013 & 3121 \\
\hline 0 & 276 & 0 & 0 & 0 \\
\hline 493 & 801 & 1242 & 1107 & 769 \\
\hline 108 & 237 & 643 & 113 & 53 \\
\hline 4483 & 4561 & 4510 & 4470 & 4510 \\
\hline 5005 & 5109 & 4500 & 5049 & 5036 \\
\hline 0 & 250 & 359 & 0 & 0 \\
\hline 4864 & 5214 & 7678 & 6116 & 6521 \\
\hline 0 & 0 & 452 & 865 & 1206 \\
\hline
\end{tabular}

(1) Formerly known as Idaho Power Company. 


$$
\text { : Page - } 58
$$

\section{SHORT-TERM IMPORTERS}

Estimated Volumes (MMCF)

TEXACO NATURAL GAS INC.

THE MEAD CORPORATION

THE MONTANA POWER COMPANY

THE MONTANA POWER TRADING \& MARKETING COMPANY TRANSCANADA GAS SERVICES INC.

TRANSCO ENERGY MARKETING COMPANY

TXU ENERGY TRADING COMPANY

UNITED STATES GYPSUM COMPANY

UPSTATE ENERGY INC.

USGEN NEW ENGLAND, INC.

UTILCORP UNITED INC.

VERMONT GAS SYSTEMS, INC.

WESTERN GAS RESOURCES, INC.

WISCONSIN GAS COMPANY

Totals

\begin{tabular}{r}
1999 \\
Jul.-Sep. \\
\hline \\
905 \\
455 \\
0 \\
4140 \\
76418 \\
7724 \\
1835 \\
929 \\
225 \\
4847 \\
1871 \\
0 \\
0 \\
2657
\end{tabular}

$\underline{549388}$

$\begin{array}{r}1999 \\ \text { Oct.Dec } \\ \hline \\ 694 \\ 455 \\ 619 \\ 5132 \\ 70328 \\ 10072 \\ 2083 \\ 923 \\ 10 \\ 2655 \\ 3694 \\ 245 \\ 0 \\ 270 \\ \\ 571348 \\ \hline\end{array}$

2000
Jan.-Mar.

$$
\begin{array}{r}
0 \\
805 \\
314 \\
5255 \\
60065 \\
8141 \\
1473 \\
874 \\
414 \\
5528 \\
3708 \\
442 \\
-\quad 0 \\
791
\end{array}
$$

$\underline{556131}$
2000
Apr.-Jun.

2000 Jui.-Sep.

0
453
0
4993
46528
12371
940
874
391
4275
2198
317
9
98

$\underline{522738}$

\section{0}

372

0

4621

62115

6213

1522

929

0

6426

3229

409

0

1825

$\underline{587258}$ 


\section{PURCHASERS OF SHORT-TERM GAS}

Estimated Volumes Imported (MMCF)

A-G Energy, L.P.

AEC Marketing (USA) Inc.

AEP Energy Services

Adams Resources Marketing, Ltd.

Alliance Energy Services

Alliance Pipeline

Altrade

Aluminum Company of America

Amerada Hess

Ameren

American Electric Power

Amoco Canada Marketing Co.

Amoco Trading

Androscoggin Energy LLC

Aquila Canada Corporation

Aquila Energy Marketing

Aquila Power Corporation

Arco Products Company

Avista Corporation

Avista Energy

BC Gas Utility

BF Goodrich

BP Energy Marketing Corp.

Barret Fuels

Barret Resources Corporation

Bay State Gas Company

Bayside Power

Big Sky Gas Marketing

Black Hills Energy

Boeing Company

Boston Gas Company

Brooklyn Union Gas

Burlington Resources Trading

CMS Gas Marketing

CXY Energy Marketing

Cabot Energy Service Corporation

Cabot Oil

CalPine Fuels Corporation

Cardinal IG Company

Cargill Energy Division

Carthage Energy Services

Cascade Natural Gas

Central Hudson Gas \& Electric

Chevron U.S.A. Inc

Cibola Corporation

City of Ellensburg, WA

City of Two Harbors, MI

Clark

Cleco

Clinton Gas Marketing
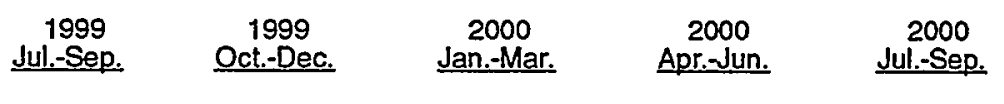$$
\begin{array}{r}
13987 \\
2 \\
4 \\
128 \\
12
\end{array}
$$$$
1286
$$$$
0
$$$$
19
$$$$
1194
$$$$
0
$$$$
3981
$$$$
143
$$$$
2351
$$$$
4139
$$$$
14255
$$$$
100
$$$$
0
$$$$
0
$$$$
9449
$$$$
0
$$$$
0
$$$$
762
$$$$
112
$$$$
233
$$$$
0
$$$$
0
$$$$
0
$$$$
2620
$$$$
1131
$$

$0 \quad 226$

$1613 \quad 1627$

0. 269

$3689 \quad 8104$

75
0

$19455 \quad 25678$

$338 \quad 1470$

$35 \quad 18$

$0 \quad 0$

$0 \quad 31$

277

$$
0
$$

13803

931

81

8

0

218

1768

0

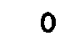

28

68

3113

289

2069

1107

0

2261

5516

9426

0

0

0

6

4243

0

491

872

0

0

341

0

101

788

1349

0

21

63

5615

34

7

27025

2182

0

0

493
362 


\section{PURCHASERS OF SHORT-TERM GAS Estimated Volumes Imported (MMCF)}

CoEnergy Trading Company

Coast Energy Group

Coastal Gas Marketing Company

Colonial Energy, Inc.

Columbia Energy Services

ComGas

Commonwealth Edison

Commonwealth Energy Services

Commonwealth Gas Company

Connecticut Natural Gas

Conoco Inc.

Consolidated Edison

Consumers Energy Company

Cook Inlet

Coral Energy Resources

Coral Redwood

Crockett Cogeneration

Delmarva Power \& Light Company

Detroit Edison Company

Duke Energy Trading \& Marketing

Duke Field Services

Duke Solutions Inc.

Dynegy Marketing \& Trade

E-Prime inc.

El Paso Energy Marketing

Encina Gas Marketing

Eneresco

Energy Masters Intemational, Inc.

Energy North Jnc.

Energy USA

Energy West Inc.

Energy West Resources

Engage Energy U.S. LP

Enline Energy Solutions

Enron Capital \& Trade Resources

Enron Intemational Gas Sales Co.

Enron North America Corp.

Enserch Energy Services, Inc.

Enserco Energy, Inc.

Equitable Resources Marketing

Essex County Gas Company

Famland Industries

Fitchburg Gas \& Electric Company

Florida Power \& Light Company

Fort James Corporation

Fuel Imbalance

Fuel Use

Gaslantic

H.Q. Energy Services (U.S.) Inc.

HL Power

\begin{tabular}{|c|c|}
\hline $\begin{array}{c}1999 \\
\text { Jul.-Sep. }\end{array}$ & $\begin{array}{c}1999 \\
\text { Oct.-Dec. }\end{array}$ \\
\hline 1079 & 417 \\
\hline 1072 & 1301 \\
\hline 11278 & 0 \\
\hline 0 & 0 \\
\hline 1822 & 1731 \\
\hline 0 & 276 \\
\hline 67 & 441 \\
\hline 0 & 50 \\
\hline 0 & 276 \\
\hline 235 & 182 \\
\hline 24 & 1 \\
\hline 2836 & 3104 \\
\hline 4799 & 1770 \\
\hline 332 & 456 \\
\hline 3671 & 3618 \\
\hline 191 & 157 \\
\hline 1999 & 3224 \\
\hline 1987 & 766 \\
\hline 1814 & 380 \\
\hline 56479 & 63905 \\
\hline 2203 & 1797 \\
\hline 0 & 0 \\
\hline 10911 & 5146 \\
\hline 0 & 0 \\
\hline 643 & 2687 \\
\hline 0 & 0 \\
\hline 0 & 0 \\
\hline 88 & 134 \\
\hline 0 & 58 \\
\hline 0 & 685 \\
\hline 460 & 200 \\
\hline 0 & 0 \\
\hline 14869 & 33438 \\
\hline 429 & 37 \\
\hline 0 & 0 \\
\hline 0 & 2713 \\
\hline 38050 & 35263 \\
\hline 120 & 0 \\
\hline 169 & 353 \\
\hline 0 & 1 \\
\hline 0 & 0 \\
\hline 847 & 193 \\
\hline 6 & 0 \\
\hline 0 & 0 \\
\hline 59 & 0 \\
\hline 0 & 945 \\
\hline 1251 & 21 \\
\hline 47 & 0 \\
\hline 1380 & 1380 \\
\hline 125 & 120 \\
\hline
\end{tabular}

2000
Apr.-Jun.

2000

Jan.-Mar.

1474

1152

1523

549

0

0
19

0

0

0
0

414

142

25

3204

979

234

2757

461

1902

0

717

83123

0

30

7127

1
4267

4267

13

5

103

97

783

0

0
28765

0

0

0
22168

72

788

38

51

0

0

1

847

0

0

1365

73

Jul.-Sep.

$$
\begin{array}{r}
515 \\
1014 \\
0 \\
2 \\
0 \\
0 \\
0 \\
0 \\
408 \\
33 \\
0 \\
3010 \\
3133 \\
130 \\
14376 \\
0 \\
2559 \\
0 \\
1516 \\
88227 \\
0 \\
0 \\
7542
\end{array}
$$$$
\begin{array}{rr}
4104 & 14376 \\
0 & 0
\end{array}
$$$$
2161 \quad 2559
$$$$
18
$$$$
3187
$$$$
79973
$$$$
0
$$$$
6818
$$$$
2
$$$$
1774
$$

80 
PURCHASERS OF SHORT-TERM GAS

Estimated Volumes Imported (MMCF)

Hesse Gas Company

1999
Jul.-Sep.

3649

Holnam Inc.

Honeymead Products Company

Howard Energy Company

IBP

IDA Corporation Energy Solutions L.P.

IEP

IGI Resources

Idacorp Energy Solutions L.P.

Idaho Power Company

Indeck Energy Services of Oswego

Indeck Energy Services of Yerkes

Intalco Aluminum Corporation

Interenergy Corporation

Intermountain Gas Company

Interstate Power Company

J. Aaron Company

K-N Gas Marketing

KN Energy

KN Marketing L.P.

Kaztec Energy

Kaztex Energy Management

KeySpan Gas East Corporation

Keyspan Ravenswood Inc.

Kimball Energy Corporation

Kimball Trading Company, L.L.C.

Koch Energy Trading

Koch Gas Services Company

Linepack

Long Island Lighting Company

Louisiana Pacific

MGI

Madison Gas \& Electric

Marathon Oil Company

Masonite Corporation

Mead Paper

Michigan Consolidated Gas Company

Michigan Energy Exchange

Mid American Natural Resources

MidAmerican Energy Company

Midland Cogeneration Venture

Mieco

Milford Power, L.P.

Minnegasco

Minnesota Com Processors

Minnesota Gas Company

Montana Power

Montana-Dakota Utilities Co.

Muphy Gas Gathering

N.Y. State Electric \& Gas

33

92

1672

34

0

0
3351

448

1103

1106

235

858

3549

0

0

32

22

325

453

605

0

27

1
270

154

0

32

71

12472

0

2

915

6861

0

0

417

47

65

182

1362

310

460

0

925

299

306

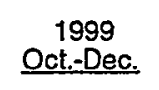

Jan.-Mar.

2000
Apr.-Jun.

2000

3667
0
92
455
34
9
0
2673

151

0

1053

1108

264

289

4000

0

0
417

125

766

0

720

375

0

208

0

369

0

800

6

13928

2

0

0

1092

5789

0

177

255

0

46

559

459

529

1409

754

1130

299

331
3623

0

91

73

17

7

0
1989

0

1096

1096

260

0

3423

0

0
34

0

84

0

1243

289

4

512

3
418

0

0
175

0

3956

0
23

0

1383

4543

0

0

427

27
0

489

0

447

1870

470

1181

296

42
3623

0

91

0

17

59

94

1299

0

1096

1096

246

0

3325

0

0
1

0

0

591

1149

0

342

1

141

131

93

0
756

0

153

0
635

5846

0

0

404

387

0

570

0

533

0

190

849

296

65
Jul.-Sep.

3642

0

92

18

16

206

1379

0

1108

1108

251

4242

96

927

0

0

0

333

466

365

53

0

0

0

0

0

61

0

0

372

5426

631

0

741

546

0

278

0

.276

0

88

845

299

63 
PURCHASERS OF SHORT-TERM GAS

Estimated Volumes Imported (MMCF)

NGX Financial Inc.

NJR Energy Services Company

NORPAC

National Fuel Gas Distribution

National Fuel Gas Supply Corporation

National Fuel Marketing Company, L.L.C.

National Fuel Resources

1999

Jul.-Sep.

1999
Oct.-Dec.

2000

2000

Apr.-Jun.

2000

(n)

National Gas \& Electric

National Gas Resources

Natural Gas Transportation Services

Nevada Power Company

New England Power Company

New Jersey Natural Gas

New York State Electric \& Gas Corporation

Niagara Mohawk

Nicor Gas Company

Nipsco Energy Trading Corporation

Noble Gas Marketing

North American Energy Inc.

Northeast Gas Markets

Northem Illinois Gas

Northem Indiana Public Service

Northem States Power (WI)

Northem States Power Company

Northshore Gas Company

Northwest Alloys

Northwest Natural Gas Company

Northwest Pacific

OGE Energy Resources

Occidental

Onyx Gas Marketing Company

Oregon Steel Mills

Oxy, Inc.

PG\&E Core Procurement Dept.

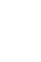

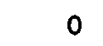

0

0

19

2172

(1)

1458

2310

464

0

82

9

4847

192

6

4197

25

842

166

0

0
103

0

$0 \quad 1645$

$5536 \quad 5364$

252.253

5863

7127

0
589
175

175
1756

146

(1458

685

0

160

0
64

2655

445

0
29

4188

0

272

234

1886

0

0

$$
\begin{aligned}
& 0 \\
& 0 \\
& 0
\end{aligned}
$$$$
106
$$

0

34

2
311

2626

0

0

258

189

0

0

1012

2347

5407

254

9376

0

1301

72

0

146

317

52436

53204

PG\&E Energy Trading

5020

2243

PG\&E Gas Transmission NW

PNGTS - Linepack

Pacific Gas \& Electric Company

Pan-Alberta Gas (U.S.) Inc.

PanEnergy Gas Services, inc.

Pancanadian Energy Services

Pancanadian Petroleum Ltd.

Pawtucket Power Associates

Peoples Gas, Light, \& Coke

Peoples Natural Gas Company

Perry Gas Company

Petrocom Energy Group Ltd.

Phoenix Chemical

Poco Marketing

81

819
2
0
175

175
0

52927

6975

50

$0 \quad 0$

34

2

0

3825

0

5809

472

0

423

3522

7958

907

108

0

753

Jul.-Sep.

Portland General Electric Company

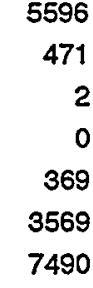


PURCHASERS OF SHORT-TERM GAS Estimated Volumes Imported (MMCF)

Potsdam College

Powerex

Proctor and Gamble Company

Progas

Proliance Energy, LLC

Public Service Gas \& Electric

Puget Sound Energy

Questar Energy Trading Co.

Questar Pipeline Company

Quick Trade

Reliant Energy Services, Inc.

Renaissance Energy (U.S.) Inc.

Rhone-Poulenc

Rochester Gas \& Electric Company

Rumford

SCANA Energy Marketing

SEMCO Energy Services

Sacramento Municipal Utility Dist.

San Diego Gas \& Electric Company

Santanna Natural Gas

Selkirk Cogeneration Partners

Sempra Energy Trading Corp.

Sierra Pacific Power Company

Sigcorp Energy Services, Inc.

Simplot Canada Limited

Siphe Power

Sithe Energy

Sonat Marketing Company

South Jersey Resources Inc.

Southem Califomia Gas Company

Southem Company Energy

Southem Connecticut Gas Company

Southem Indiana

Southem Indiana Gas

Southwest Gas Corporation

Sprague Energy

St. Lawrence Gas

St. Lawrence University

Stalwart Energy Company

Star Natural Gas Company

Statoil Energy Services, Inc.

Suncor, inc.

TM Star

TXU Energy Trading Company

Tejas Power

Tenaska Marketing Ventures

Tenaska Washington Partners

Tesoro Northwest Company

TexEnergy

Texaco Energy Marketing

\begin{tabular}{|c|c|c|c|}
\hline $\begin{array}{c}1999 \\
\text { Jul.-Sep. }\end{array}$ & $\begin{array}{c}1999 \\
\text { Oct.-Dec. }\end{array}$ & $\begin{array}{c}2000 \\
\text { Jan.-Mar. }\end{array}$ & $\begin{array}{c}2000 \\
\text { Apr.Jun. }\end{array}$ \\
\hline
\end{tabular}

\begin{tabular}{|c|c|c|c|c|}
\hline 51 & 49 & 47 & 47 & 47 \\
\hline 0 & 0 & 0 & 0 & 21 \\
\hline 1200 & 201 & 2343 & 5448 & 5625 \\
\hline 0 & 0 & 0 & 135 & 13 \\
\hline 0 & 0 & 0 & 120 & 93 \\
\hline 1919 & 61 & 0 & 0 & 0 \\
\hline 9442 & 10940 & 12276 & 10736 & 16542 \\
\hline 183 & 5 & 0 & 0 & 1 \\
\hline 0 & 0 & 0 & 0 & 2 \\
\hline 735 & 156 & 5 & 64 & 337 \\
\hline 8138 & 10370 & 8390 & 9600 & 10438 \\
\hline 13469 & 10061 & 8853 & 9343 & 13180 \\
\hline 0 & 12 & 12 & 0 & 0 \\
\hline 2514 & 3004 & 6194 & 3494 & 2526 \\
\hline 0 & 0 & 0 & 0 & 88 \\
\hline 25 & 65 & 316 & 90 & 927 \\
\hline 153 & 0 & 0 & 0 & 0 \\
\hline 844 & 171 & 3 & 1178 & 1214 \\
\hline 2062 & 1930 & 2581 & 2179 & 1332 \\
\hline 0 & 0 & 311 & 0 & 0 \\
\hline 20 & 0 & 33 & 0 & 0 \\
\hline 5063 & 2036 & 2320 & 2457 & 2254 \\
\hline 3694 & 5101 & 6011 & 4349 & 3842 \\
\hline 0 & 0 & 0 & 0 & 157 \\
\hline 0 & 0 & 0 & 0 & 262 \\
\hline 0 & 0 & 0 & 118 & 0 \\
\hline 0 & 0 & 37 & 62 & 277 \\
\hline 62 & 0 & 0 & 0 & 0 \\
\hline 312 & 0 & 0 & 0 & 20 \\
\hline 16 & 3 & 0 & 6 & 27 \\
\hline 4104 & 6538 & 4836 & 5347 & 3170 \\
\hline 0 & 386 & 68 & 66 & 0 \\
\hline 31 & 42 & 29 & 1100 & 0 \\
\hline 0 & 0 & 0 & 0 & 868 \\
\hline 379 & 745 & 931 & 346 & 358 \\
\hline 0 & 0 & 0 & 0 & 9 \\
\hline 0 & 0 & 14 & 0 & 0 \\
\hline 35 & 12 & 0 & 0 & 0 \\
\hline 0 & 0 & 26 & 0 & 0 \\
\hline . 302 & 93 & 122 & 0 & 0 \\
\hline 592 & 2715 & 304 & 1619 & 407 \\
\hline 0 & 0 & 1 & 0 & 0 \\
\hline 0 & 0 & 47 & 21 & 0 \\
\hline 1841 & 2948 & 1265 & 661 & 833 \\
\hline 27 & 205 & 0 & 0 &.$\quad 0$ \\
\hline 3758 & 5734 & 7414 & 6815 & 8285 \\
\hline 103 & 321 & 0 & 0 & 40 \\
\hline 660 & 892 & 954 & 574 & 462 \\
\hline 0 & 16 & 0 & 0 & 0 \\
\hline 0 & 0 & 496 & 865 & 1206 \\
\hline
\end{tabular}


PURCHASERS OF SHORT-TERM GAS Estimated Volumes Imported (MMCF)

Texaco Natural Gas

Texex Energy Partners

Torch Gas L.C.

Tosco Refining Company

Tractebel

TransCanada Energy Marketing USA

TransCanada Gas Services

TransCanada Power (Castleton) LLC

U.S. Energy Services

U.S. Gas Transportation Inc.

U.S. Generating Company

Unicom Energy Ohio, Inc.

United States Gypsum

Unocal

Upstate Energy, Inc.

Utilicom United

Valley Gas Company

Various CA State Markets

Various CO State Markets

Various CT State Markets

Various IA State Markets

Various IL State Markets

Various LA State Markets

Various MA State Markets

Various ME State Markets

Various MI State Markets

Various MN State Markets

Various MT State Markets

Various NE State Markets

Various NJ State Markets

Various NV State Markets

Various NY State Markets

Various OR State Markets

Various WA State Markets

Various WI State Markets

Vermont Gas Systems, Inc.

Virginia Power

WICOR Energy Service Company

WP Natural Gas

WPS Energy Services, Inc.

WSU

West Linn Paper Company

Western Gas Marketing Inc.

Western Gas Resources

Western Gas Services

Weyerhauser

Wild Goose Storage

Willamette

Williams Energy Company

Wisconsin Electric

\begin{tabular}{|c|c|c|c|c|}
\hline $\begin{array}{c}1999 \\
\text { Jul.-Sep. }\end{array}$ & $\begin{array}{c}1999 \\
\text { Oct.-Dec. }\end{array}$ & $\begin{array}{c}2000 \\
\text { Jan.-Mar. }\end{array}$ & $\begin{array}{c}2000 \\
\text { Apr.-Jun. }\end{array}$ & $\begin{array}{c}2000 \\
\text { Jul.-Sep. }\end{array}$ \\
\hline
\end{tabular}

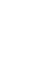

108

455

1639

2270

383

803

34

16

0
929

744

1117

3287

0
13471

2992

0

4785

18813

0

5906

0
2606

122

48

316

149

920

15365

66

1488

118

0

30

0
709

1185

0

59

0

455

5

733

43

760

406

116
49
562

283

0
1513

3239

73

1057

161

32

0
923

336

10

5446

0

13185

0

4373

16123

0

5902

0
659

2068

3969

0

58

507

23820

964

1739

420

245

6

0

443

1012

$$
0
$$

0

9

92

0

639

16

338

309

49
864

29

0

249

Apr.-Jun.

Jul.-Sep.

0
911

5885

0

1327

34

0

0
876

363

433

5287

79

11786

3785

0

5627

17286

3069

4452

4337

5

3442

0
0

2

449

26600

865

1451

- 32

442

0

0

626

823

0

0

23

250

0

546

29

400

214

41

$\begin{array}{rr}45 & 544 \\ 39 & 23 \\ 0 & 0 \\ 224 & 124 \\ 0 & 0 \\ 565 & 1253\end{array}$

$1593 \quad 264$

$0 \quad 0$

$698 \quad 714$

$44 \quad 75$

8410

8919973

$296 \quad 508$

4350

$3089 \quad 5030$

$\begin{array}{rr}0 & 0 \\ 7544 & 12770\end{array}$

$0 \quad 3124$

$27 \quad 10$

$5634 \quad 5396$

$19668 \quad 17179$

$22604 \quad 29311$

$162 \quad 216$

$10474 \quad 12023$

$\begin{array}{rr}0 & 0 \\ 976 & 1952\end{array}$

3571

0

$$
2
$$

31850

0

1279

110

317

0

62

0

2184

0

0

0

901

0

0
15

1045

1660

40

0

0

0

0

22655

0

1702

33

409

0

218

0

1842

42

0

12

0

442 .

10

. 0

400
44

0

0

0

0 24

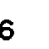
.

0 
Page - 65

PURCHASERS OF SHORT-TERM GAS

Estimated Volumes Imported (MMCF)

Wisconsin Fuel \& Light

Wisconsin Gas

Wisconsin Power \& Light

Wisconsin Public Service Corp.

Yankee Gas Services

Totals

\begin{tabular}{|c|c|c|c|c|}
\hline $\begin{array}{r}1999 \\
\text { Jul.-Sep. }\end{array}$ & $\begin{array}{c}1999 \\
\text { Oct.-Dec. }\end{array}$ & $\begin{array}{c}2000 \\
\text { Jan.-Mar. }\end{array}$ & $\begin{array}{c}2000 \\
\text { Apr.-Jun. }\end{array}$ & $\begin{array}{r}2000 \\
\text { Jul.-Sep. }\end{array}$ \\
\hline 0 & 18 & 0 & 0 & 18 \\
\hline 4432 & 1600 & 952 & 173 & 2651 \\
\hline 1307 & 1078 & 610 & 185 & 563 \\
\hline 735 & 252 & 0 & 0 & 0 \\
\hline 870 & 577 & 35 & 0 & 0 \\
\hline$\underline{549388}$ & $\underline{571348}$ & $\underline{556131}$ & $\underline{522738}$ & 587258 \\
\hline
\end{tabular}




\section{SHORT-TERM SALES \\ YEAR: 2000 Quarter: Three \\ Estimated Volumes (MMCF) \& Prices (\$ / MMBTU)}

Importer
Seller

Purchaser / End User

AEC MARKETING (USA) INC.

AEC Marketing

AEC Marketing (USA) Inc.

AEC WEST LTD.

AEC West Ltd.

AEC Marketing (USA) Inc.

Point

of

Eastport, Idaho

Port of Morgan, MT

Port of Morgan, MT

Massena, New York

Sherwood, ND

AMOCO CANADA MARKETING CORPORATION

Amoco Canada Petroleum Company Ltd.

AEP Energy Services

Eastport, Idaho

AMOCO CANADA MARKETING CORPORATION

Amoco Canada Petroleum Company Ltd.

Altrade

Niagara Falls, NY

AMOCO CANADA MARKETING CORPORATION

Amoco Canada Petroleum Company Ltd.

American Electric Power

Eastport, Idaho

AMOCO CANADA MARKETING CORPORATION

Amoco Canada Petroleum Company Ltd.

Amoco Trading

Calais, ME

AMOCO CANADA MARKETING CORPORATION

Amoco Canada Petroleum Company Ltd.

Amoco Trading

Eastport, Idaho

AMOCO CANADA MARKETING CORPORATION

Amoco Canada Petroleum Company Ltd.

Amoco Trading

Waddington, NY

o N/A

$50 \quad 4.66$

o N/A

$50 \quad 4.66$

AMOCO CANADA MARKETING CORPORATION

Amoco Canada Petroleum Company Ltd.

Arco Products Company

Vol. ${ }^{\text {July }}$ Price

August

Vol. Price

September

Vol. Price

Quarterly

Total

Vol. ${ }^{\text {Avg. }}$ Price

$2165 \quad 2.50$

$2566 \quad 2.18$

2488

2.18

7219

2.28

$1854-250$

2388

2.18

$2444 \quad 2.18$

6686

2.27

$313 \quad 3.92$

$313 \quad 3.48$

$307 \quad 3.46$

932

3.62

$158 \quad 4.94$

172

4.11

151

4.89

482

4.63

o N/A

$800 \quad 3.57$

$3820 \quad 4.56$

4620

4.39

o N/A

- N/A

$18 \quad 5.54$

$18 \quad 5.54$

- N/A

o N/A

$16 \quad 3.36$

16

3.36

$69 \quad 5.30$

- N/A

o N/A

69

5.30

931

$344 \quad 5.30$

543

8.70

o N/A

887

7.38

Sumas, Washington

$357 \quad 2.96$

o N/A 


\section{SHORT-TERM SALES}

YEAR: 2000 Quarter: Three

Estimated Volumes (MMCF) \& Prices (\$/MMBTU)

Importer

Seller

Purchaser / End User

AMOCO CANADA MARKETING CORPORATION

Amoco Canada Petroleum Company Ltd.

BP Energy Marketing Corp.

Calais, ME

AMOCO CANADA MARKETING CORPORATION

Amoco Canada Petroleum Company Ltd.

BP Energy Marketing Corp.

Eastport, Idaho

AMOCO CANADA MARKETING CORPORATION

Amoco Canada Petroleum Company Lid.

BP Energy Marketing Corp.

Niagara Falls, NY

AMOCO CANADA MARKETING CORPORATION

Amoco Canada Petroleum Company Ltd.

BP Energy Marketing Corp.

Port of Morgan, MT

AMOCO CANADA MARKETING CORPORATION

Amoco Canada Petroleum Company Ltd.

BP Energy Marketing Corp.

Waddington, NY

- N/A

O N/A

1

5.57

$1 \quad 5.57$

AMOCO CANADA MARKETING CORPORATION

Amoco Canada Pelroleum Company Ltd.

Barret Resources Corporation

Sumas, Washington

AMOCO CANADA MARKETING CORPORATION

Amoco Canada Petroleum Company Ltd.

Bayside Power

Calais, ME

229

3.97

$199 \quad 3.61$

$0 \quad$ N/A

428

3.80

AMOCO CANADA MARKETING CORPORATION

Amoco Canada Petroleum Company Ltd.

Coast Energy Group

Eastport, Idaho

$12 \quad 5.30$

o N/A

$14 \quad 5.54$

$26 \quad 5.43$

AMOCO CANADA MARKETING CORPORATION

Amoco Canada Petroleum Company Ltd.

Consolidated Edison

Niagara Falls, NY

1014

4.86

101

4.84

98

3.36

$3010 \quad 4.36$

AMOCO CANADA MARKETING CORPORATION

Amoco Canada Petroleum Company Ltd.

Cook Inlet

Eastport, Idaho

$61 \quad 5.30$

$0 \quad$ N/A

o N/A

$61 \quad 5.30$

AMOCO CANADA MARKETING CORPORATION

Amoco Canada Petroleum Company Ltd.

Cook Inlet

Sumas, Washington

AMOCO CANADA MARKETING CORPORATION

Amoco Canada Petroleum Company Ltd.

Coral Energy Resources
Eastport, Idaho
$0 \quad$ N/A

$6 \quad 5.54$ 


\section{SHORT-TERM SALES \\ YEAR: 2000 Quarter: Three \\ Estimated Volumes (MMCF) \& Prices (\$ / MMBTU)}

\begin{tabular}{ll} 
Importer & Point \\
Seller & of \\
Purchaser / End User & Entry \\
\hline
\end{tabular}

AMOCO CANADA MARKETING CORPORATION

Amoco Canada Petroleum Company Ltd.

Coral Energy Resources

Sumas, Washington

AMOCO CANADA MARKETING CORPORATION

Amoco Canada Petroleum Company Ltd.

Crockett Cogeneration

Eastport, Idaho

AMOCO CANADA MARKETING CORPORATION

Amoco Canada Petroleum Company Ltd.

Duke Energy Trading \& Marketing

Eastport, Idaho

$24 \quad 5.30$

O N/A

$0 \quad N / A$

$24 \quad 5.30$

AMOCO CANADA MARKETING CORPORATION

Amoco Canada Petroleum Company Ltd.

Duke Energy Trading \& Marketing

Sumas, Washington

AMOCO CANADA MARKETING CORPORATION

Amoco Canada Petroleum Company Ltd.

Engage Energy U.S. LP

Calais, ME

AMOCO CANADA MARKETING CORPORATION

Amoco Canada Petroleum Company Ltd.

Enron North America Corp.

Sumas, Washington

AMOCO CANADA MARKETING CORPORATION

Amoco Canada Petroleum Company Ltd.

Enserco Energy, inc.

Eastport, ldaho

- N/A

N/A

$3 \quad 5.54$

$3 \quad 5.54$

AMOCO CANADA MARKETING CORPORATION

Amoco Canada Petroleum Company Ltd.

IGI Resources

Sumas, Washington

o N/A

- N/A

$\begin{array}{ll}78 & 3.53\end{array}$

$\begin{array}{ll}78 & 3.53\end{array}$

AMOCO CANADA MARKETING CORPORATION

Amoco Canada Petroleum Company Ltd.

NGX Financial Inc.

Niagara Falls, NY

- N/A

- N/A

$11 \quad 3.36$

11

3.36

AMOCO CANADA MARKETING CORPORATION

Amoco Canada Petroleum Company Ltd.

Northem States Power (WI)

Noyes, Minnesota

- N/A

O N/A

181

2.24

181

2.24

AMOCO CANADA MARKETING CORPORATION

Amoco Canada Petroleum Company Ltd.

Northwest Natural Gas Company

Sumas, Washington

$271 \quad 3.74$

$272 \quad 2.96$

$264 \quad 3.53$

807

3.41

AMOCO CANADA MARKETING CORPORATION

Amoco Canada Petroleum Company Ltd.

Pacific Gas \& Electric Company
Eastport, Idaho
$24 \quad 5.30$

$0 \quad$ N/A
$3 \quad 5.54$

27

5.32 


\section{SHORT-TERM SALES}

YEAR: 2000 Quarter: Three

Estimated Volumes (MMCF) \& Prices (\$ / MMBTU)

Importer

Seller

Purchaser / End User

AMOCO CANADA MARKETING CORPORATION

Amoco Canada Petroleum Company Ltd.

PanEnergy Gas Services, Inc.

Eastport, Idaho

AMOCO CANADA MARKETING CORPORATION

Amoco Canada Petroleum Company Ltd.

Powerex

Sumas, Washington

AMOCO CANADA MARKETING CORPORATION

Amoco Canada Petroleum Company Ltd.

Progas

St. Clair, Michigan

AMOCO CANADA MARKETING CORPORATION

Amoco Canada Petroleum Company Ltd.

Puget Sound Energy

Sumas, Washington

AMOCO CANADA MARKETING CORPORATION

Amoco Canada Petroleum Company Ltd.

Reliant Energy Services, Inc.

Eastport, Idaho

$6 \quad 5.30$

75

8.70

$0 \quad$ N/A

74

8.70

o N/A

$0 \quad$ N/A

262

3.46

262

3.46

AMOCO CANADA MARKETING CORPORATION

Amoco Canada Petroleum Company Ltd.

Southem Company Energy

Eastport, Idaho

$501 \quad 5.30$

290

8.70

405

5.54

1195

6.21

AMOCO CANADA MARKETING CORPORATION

Amoco Canada Petroleum Company Ltd.

TXU Energy Trading Company

Eastport, Idaho

$68 \quad 5.30$

$40 \quad 8.70$

$0 \quad$ N/A

108

6.57

AMOCO CANADA MARKETING CORPORATION

Amoco Canada Petroleum Company Ltd.

U.S. Gas Transportation Inc.

Eastport, Idaho

$48 \quad 5.30$

O N/A

- N/A

48

5.30

AMOCO CANADA MARKETING CORPORATION

Amoco Canada Petroleum Company Ltd.

U.S. Gas Transportation Inc.

Sumas, Washington

$0 \quad$ N/A

- N/A

26

3.53

26

3.53

AMOCO ENERGY TRADING CORP. (LNG)

Amoco Energy Trading Corporation

Amoco Trading

Lake Charles, LA $\begin{array}{llll}0 & \text { N/A } & 1805 & 3.80\end{array}$

o N/A

$1805 \quad 3.80$ 


\section{SHORT-TERM SALES \\ YEAR: 2000 Quarter: Three \\ Estimated Volumes (MMCF) \& Prices (\$ / MMBTU)}

Importer

Seller

Purchaser / End User

Point

Of

Pittsburg, NH

Pittsburg, NH

Port of Morgan, MT

Aquila Canada Corporation

Duke Energy Trading \& Marketing

ANDROSCOGGIN ENERGY LLC

ANDROSCOGGIN ENERGY LLC

Sempra Energy Trading

Androscoggin Energy LLC
Coral Energy Canada Inc.

July
Vol. Pugust
Pol. Price

Quarterly

Total

Vol. ${ }^{\text {Avg. }}$ Price

o N/A

$7 \quad 4.89$

$18 \quad 5.25$

25

5.15

AQUILA ENERGY MARKETING CORPORATION

Aquila Canada Corporation

Enron North America Corp.

Eastport, Idaho

$1714 \quad 3.86$

O N/A

108

5.26

$2801 \quad 4.40$

AQUILA ENERGY MARKETING CORPORATION

Aquila Canada Corporation

Keyspan Ravenswood lnc.

Niagara Falls, NY

- N/A

$341 \quad 3.95$

- N/A

341

3.95

AQUILA ENERGY MARKETING CORPORATION

Aquila Canada Corporation

MidAmerican Energy Company

Port of Morgan, MT

o N/A

o N/A

300

4.70

300

4.70

AQUILA ENERGY MARKETING CORPORATION

Aquila Canada Corporation

Portland General Electric Company

Sumas, Washington

$56 \quad 5.10$

103

3.12

- N/A

159

3.82

AQUILA ENERGY MARKETING CORPORATION

Aquila Canada Corporation

Southem Company Energy

Eastport, Idaho

$0 \quad$ N/A

$712 \quad 3: 94$

O N/A

712

3.94

AQUILA ENERGY MARKETING CORPORATION

Aquila Canada Corporation

Southem Company Energy

Port of Morgan, MT

$309 \quad 4.44$

O N/A

$0 \quad$ N/A

309

4.44

AQUILA ENERGY MARKETING CORPORATION

Aquila Canada Corporation

Southwest Gas Corporation

Sumas, Washington

$66 \quad 5.10$

103

3.12

$94 \quad 3.47$

263

3.74

ARCO PRODUCTS COMPANY

Amoco Oil Company

Arco Products Company

Sumas, Washington

$316 \quad 3.68$

326

3.10

$388 \quad 3.81$

1030

3.55

ARCO PRODUCTS COMPANY

Duke Energy Marketing Canada

Arco Products Company $\begin{array}{llll}90 & 3.61 & 90 & 3.68\end{array}$

$87 \quad 3.49$

266

3.59 


\section{SHORT-TERM SALES \\ YEAR: 2000 Quarter: Three \\ Estimated Volumes (MMCF) \& Prices (\$ / MMBTU)}

Importer

Seller

Purchaser / End User

ARCO PRODUCTS COMPANY

Engage Energy Canada

Arco Products Company

AVISTA CORPORATION

Avista Energy Inc.

Avista Corporation

AVISTA CORPORATION

Engage Energy Canada

Avista Corporation

AVISTA CORPORATION

Enron Gas Marketing Canada

Avista Corporation

AVISTA CORPORATION

PG\&E Energy Trading

Avista Corporation

AVISTA ENERGY, INC.

Barrett Resources

El Paso Energy Marketing

AVISTA ENERGY, INC.

CanWest Gas Supply Inc.

Clark

AVISTA ENERGY, INC.

Canadian Natural Resources Limited

Avista Corporation

AVISTA ENERGY, INC.

Canadian Natural Resources Limited

J. Aaron Company

AVISTA ENERGY, INC.

Coral Energy Canada Inc.

Sierra Pacific Power Company

AVISTA ENERGY, INC.

Dynegy Canada

CMS Gas Marketing

AVISTA ENERGY, INC.

Engage Energy Canada

Avista Corporation
Point

Of

Sumas, Washington

$137 \quad 3.62$

$113 \quad 3.10$

$87 \quad 3.49$

$337 \quad 3.41$

Eastport, Idaho

$182 \quad 3.65$

o N/A

$0 \quad$ N/A

$182 \quad 3.65$

Eastport, Idaho

$308 \quad 3.76$

$162 \quad 3.33$

$297 \quad 3.67$

767

3.63

Eastport, Idaho

308

Eastport, Idaho

$857 \quad 3.75$

$0 \quad$ N/A

$442 \quad 3.60$

1299

3.70

Sumas, Washington

$153 \quad 4.09$

O N/A

$0 \quad$ N/A

$153 \quad 4.09$

Sumas, Washington

$177 \quad 2.52$

o N/A

- N/A

$177 \quad 2.52$

Sumas, Washington

$548 \quad 3.75$

o N/A

$0 \quad$ N/A

548

3.75

Sumas, Washington

- N/A

477

3.02

450

3.51

927

3.26

Sumas, Washington

- N/A

152

2.95

- N/A

152

2.95

Sumas, Washington

- N/A

154

2.90

- N/A

154

2.90

Sumas, Washington
586

3.16 


\section{SHORT-TERM SALES \\ YEAR: 2000 Quarter: Three \\ Estimated Volumes (MMCF) \& Prices (\$ / MMBTU)}

Importer

Purchaser / End User

AVISTA ENERGY, INC.

Engage Energy Canada

Clark

AVISTA ENERGY, INC.

Engage Energy Canada

WSU

AVISTA ENERGY, INC.

Enron Gas Marketing Canada

IEP

AVISTA ENERGY, INC.

Numac Energy

BF Goodrich

AVISTA ENERGY, INC.

Numac Energy

Southwest Gas Corporation

AVISTA ENERGY, INC.

PG\&E Energy Trading

Avista Corporation

Eastport, Idaho

Eastport, Idaho

- N/A

- N/A

$567 \quad 2.21$

$567 \quad 2.21$

AVISTA ENERGY, INC.

PG\&E Energy Trading

Dynegy Marketing \& Trade

AVISTA ENERGY, INC.

Petro-Canada Hydrocarbons Inc.

Avista Comoration

AVISTA ENERGY, INC.

Petro-Canada Hydrocarbons Inc.

Clark

Sumas, Washington

- N/A

310

3.13

- N/A

310

3.13

AVISTA ENERGY, INC.

Petro-Canada Hydrocarbons Inc.

Statoil Energy Services, Inc.

Sumas, Washington

$325 \quad 3.58$

$0 \quad$ N/A

o N/A

325

3.58

AVISTA ENERGY, INC.

Sempra Energy Trading

IEP 


\section{SHORT-TERM SALES}

YEAR: 2000 Quarter: Three

Estimated Volumes (MMCF) \& Prices ( $\$$ /MMBTU)

Importer

Seller

Purchaser / End User

BARRINGTON PETROLEUM LTD.

Barrington Petroleum Limited

Enron North America Corp.

BC GAS UTILITY LTD.

B.C. Gas Inc.

Duke Energy Trading \& Marketing

BC GAS UTILITY LTD.

B.C. Gas Inc.

Enron North America Corp.

BC GAS UTILITY LTD.

B.C. Gas Inc.

Puget Sound Energy

BIG SKY GAS MARKETING

Engage Energy Canada

Big Sky Gas Marketing

BURLINGTON RESOURCES TRADING INC.

Burlington Resources Canada Energy LTD

Burlington Resources Trading

CANWEST GAS SUPPLY U.S.A., INC.

CanWest Gas Supply Inc.

Coral Energy Resources

CANWEST GAS SUPPLY U.S.A., INC.

CanWest Gas Supply Inc.

Questar Energy Trading Co.

CANWEST GAS SUPPLY U.S.A., INC.

CanWest Gas Supply Inc.

Sierra Pacific Power Company

CANWEST GAS SUPPLY U.S.A., INC.

CanWest Gas Supply inc.

Southem Company Energy

CANWEST GAS SUPPLY U.S.A., INC.

CanWest Gas Supply Inc.

Southwest Gas Comporation

CASCADE NATURAL GAS CORPORATION

Duke Energy Marketing Canada

Cascade Natural Gas
Point

Entry

Port of Morgan, MT

Sumas, Washington

Sumas, Washington

Sumas, Washington

Noyes, Minnesota

Eastport, Idaho

Sumas, Washington

Sumas, Washington

Sumas, Washington

Sumas, Washington

Sumas, Washington

Eastport, Idaho

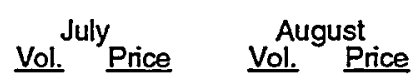

September

Vol. Price

Quarterly

Total

Vol. ${ }^{\text {Avg. }}$ Price

$151 \quad 4.51$

$151 \quad 3.71$

$147 \quad 4.62$

449

4.28

$62 \quad 3.20$

o N/A

O N/A

$62 \quad 3.20$

$49 \quad 3.15$

$8 \quad 2.92$

- N/A

58

3.12

$23 \quad 3.12$

$\begin{array}{ll}75 & 3.20\end{array}$

$0 \quad$ N/A

$98 \quad 3.18$

257

4.31

$257 \quad 3.52$

$249 \quad 4.39$

$764 \quad 4.07$

236

$$
3.94
$$

2398

3.83

$2303 \quad 5.23$

7070

4.32

$234 \quad 3.94$

- N/A

o N/A

234

3.94

$1 \quad 3.07$

- N/A

- N/A

$1 \quad 3.07$

$28 \quad 3.20$

O N/A

- N/A

$28 \quad 3.20$

$\begin{array}{llll}1 & 3.19 & 139 & 3.27\end{array}$

$0 \quad$ N/A

$140 \quad 3.27$

$35 \quad 3.33$

- N/A

N/A

35

3.33

o N/A

o N/A

352

352

3.51 


\section{SHORT-TERM SALES}

YEAR: 2000 Quarter: Three

Estimated Volumes (MMCF) \& Prices (\$ / MMBTU)

Importer

Seller

Purchaser/End User .

CASCADE NATURAL GAS CORPORATION

Duke Energy Marketing Canada

Cascade Natural Gas

CASCADE NATURAL GAS CORPORATION

\section{E-Prime}

Cascade Natural Gas

Point
of
Entry

Sumas, Washington

Sumas, Washington

Eastport, Idaho

Sumas, Washington

Eastport, Idaho

Chevron Canada Resources

Dynegy Marketing \& Trade

CASCADE NATURAL GAS CORPORATION

Cascade Natural Gas

CASCADE NATURAL GAS CORPORATION

Sempra Energy Trading $\begin{array}{lll}\text { July } & \begin{array}{l}\text { August } \\ \text { Vol. }\end{array} \text { Price September } \\ \text { Vol. Price Price }\end{array}$

Quarterly

Total

Vol. ${ }^{\text {Avg. }}$ Price

Engage Energy Canada

$164 \quad 4.00$

$815 \quad 3.23$

$551 \quad 3.43$

1530

3.38

CMS MARKETING SERVICES AND TRADING COMPANY

Nigeria LNG Ltd. (Nigeria)

Various LA State Markets

Lake Charles, LA

$2496 \quad 4.37$

O N/A

$0 \quad$ N/A

2496

4.37

CMS MARKETING SERVICES AND TRADING COMPANY

Northwest Shelf LNG Sellers (Australia)

Various LA State Markets

Lake Charles, LA

$2285 \quad 2.78$

$0 \quad$ N/A

1270

2.80

3555

2.79

CMS MARKETING SERVICES AND TRADING COMPANY

Qatar General Petroleum Corp.

Various LA State Markets

Lake Chartes, LA

$0 \quad$ N/A

$0 \quad$ N/A

$2744 \quad 3.95$

2744

3.95

CMS MARKETING SERVICES AND TRADING COMPANY

Qatar Liquefied Gas Co. Ltd. (Qatar)

Various LA State Markets

Lake Charles, LA

O N/A

4780

2.71

$2385 \quad 2.49$

7165

2.63

CMS MARKETING SERVICES AND TRADING COMPANY

Ras Laffan Liquefied Nat. Gas Co. (Qatar)

Various LA State Markets

Lake Charles, LA

$4793 \quad 3.53$

2387

2.9

249

4.07

9675

3.53

COAST ENERGY GROUP

Coast Energy Canada

Various CA State Markets

Eastport, Idaho

$518 \quad 2.85$

804

3.37

$604 \quad 4.96$

1927

3.73

COENERGY TRADING COMPANY

Coral Energy Canada Inc.

CoEnergy Trading Company

Grand Island, NY
$58 \quad 3.98$

$70 \quad 4.80$

131 


\section{SHORT-TERM SALES}

YEAR: 2000 Quarter: Three

Estimated Volumes (MMCF) \& Prices (\$/MMBTU)

Importer

Seller

Purchaser / End User

COENERGY TRADING COMPANY

Coral Energy Canada Inc.

CoEnergy Trading Company

COENERGY TRADING COMPANY

Renaissance Energy Ltd.

CoEnergy Trading Company

\section{COMMONWEALTH GAS COMPANY}

ANE .

Commonwealth Gas Company

CORAL ENERGY RESOURCES, L.P. Coral Energy Canada Inc.

City of Ellensburg, WA

CORAL ENERGY RESOURCES, L.P.

Coral Energy Canada inc.

Michigan Consolidated Gas Company

CORAL ENERGY RESOURCES, L.P. Coral Energy Canada Inc.

National Fuel Resources

CORAL ENERGY RESOURCES, L.P. Coral Energy Canada Inc.

Perry Gas Company

CORAL ENERGY RESOURCES, L.P.

Coral Energy Canada Inc.

Proctor and Gamble Company

CORAL ENERGY RESOURCES, L.P.

Coral Energy Canada Inc.

Rumford

CORAL ENERGY RESOURCES, L.P. Coral Energy Canada lnc.

Sithe Energy

CORAL ENERGY RESOURCES, L.P. Coral Energy Canada Inc.

Various MN State Markets

CORAL ENERGY RESOURCES, L.P. Coral Energy Canada inc.

Various NY State Markets
Point

Entry

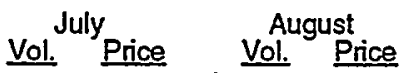

September

Vol. Price

Quarteriy

Total

Vol. Prg. Price

Niagara Falls, NY

$\begin{array}{llll}0 & \text { N/A } & 75 & 3.94\end{array}$

- N/A

75

3.94

Grand Island, NY

$34 \quad 4.43$

o N/A

$0 \quad$ N/A

34

4.43

Waddington, NY

$138 \quad 4.59$

138

3.86

133

4.83

408

4.42

Sumas, Washington

$231 \quad 3.88$

$2 \quad 3.16$

$201 \quad 4.53$

435

4.18

Noyes, Minnesota

$186 \quad 2.14$

$186 \quad 2.14$

180

2.14

552

2.14

Grand Island, NY

$60 \quad 4.12$

$26 \quad 4.08$

$16 \quad 5.95$

$102 \quad 4.40$

Champlain, NY

04.65 .

$0 \quad 4.10$

$0 \quad 4.69$

14.55

Niagara Falls, NY

$2383 \quad 2.83$

1687

Pittsburg, NH

$0 \quad$ N/A

$30 \quad 4.75$

58

5.10

$88 \quad 4.98$

Massena, New York

$19 \quad 4.28$

$61 \quad 4.57$

N N/A

$80 \quad 4.50$

Noyes, Minnesota

$423 \quad 2.79$

198

207

4.73

$828 \quad 3.57$

Waddington, NY $\begin{array}{llllllll}991 & 4.08 & 204 & 4.28 & 166 & 5.37 & 1361 & 4.27\end{array}$ 


\section{SHORT-TERM SALES}

YEAR: 2000 Quarter: Three

Estimated Volumes (MMCF) \& Prices (\$/MMBTU)

Importer

Purchaser / End User

CORAL ENERGY RESOURCES, L.P.

Coral Energy Canada Inc.

Wisconsin Gas

CORAL ENERGY RESOURCES, L.P. (LNG)

Nigeria LNG Ltd. (Nigeria)

Coral Energy Resources

CORAL ENERGY RESOURCES, L.P. (LNG)

Oman LNG

Coral Energy Resources
Point
of
Entry

Noyes, Minnesota

Lake Charles, LA
Vol. ${ }^{\text {July }}$ Price $\begin{gathered}\text { August } \\ \text { Vol. Price }\end{gathered}$

September
Vol. Price

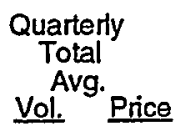

150

3.80

O N/A

o N/A

$150 \quad 3.80$

- N/A

$2510 \quad 3.20$

$2658 \quad 3.17$

5168

3.18

Lake Charles, LA

$2464 \quad 2.44$

$2461 \quad 2.44$

$0 \quad$ N/A

$4925 \quad 2.44$

CRESTAR ENERGY MARKETING CORPORATION

Crestar Energy

N.Y. State Electric \& Gas

Niagara Falls, NY

- N/A

$5 \quad 5.00$

$58 \quad 5.46$

$63 \quad 5.42$

CXY ENERGY MARKETING (U.S.A.) INC.

CXY Marketing

AEP Energy Services

Noyes, Minnesota

- N/A

$306 \quad 3.93$

$296 \quad 4.73$

$602 \quad 4.32$

CXY ENERGY MARKETING (U.S.A.) INC.

CXY Marketing

Altrade

Eastport, Idaho

o N/A

$0 \quad$ N/A

$134 \quad 4.60$

$134 \quad 4.60$

CXY ENERGY MARKETING (U.S.A.) INC.

CXY Marketing

Amoco Canada Marketing Co.

Eastport, Idaho

$64 \quad 3.26$

o N/A

- N/A

$64 \quad 3.26$

CXY ENERGY MARKETING (U.S.A.) INC.

CXY Marketing

Aquila Energy Marketing

Noyes, Minnesota

- N/A

$38 \quad 4.44$

$0 \quad$ N/A

$38 \quad 4.44$

CXY ENERGY MARKETING (U.S.A.) INC.

CXY Marketing

BP Energy Marketing Corp.

Eastport, Idaho

$0 \quad$ N/A

$441 \quad 3.22$

$304 \quad 4.53$

$745 \quad 3.75$

CXY ENERGY MARKETING (U.S.A.) INC.

CXY Marketing

Barret Resources Corporation

Eastport, Idaho

$347 \quad 3.63$

- N/A

$0 \quad$ N/A

$347 \quad 3.63$

CXY ENERGY MARKETING (U.S.A.) INC.

CXY Marketing

CMS Gas Marketing

Noyes, Minnesota

$\begin{array}{llll}0 & \text { N/A } & 155 & 3.95\end{array}$

- N/A

155

3.95

CXY ENERGY MARKETING (U.S.A.) INC.

CXY Marketing

Coral Energy Resources

Noyes, Minnesota
$92 \quad 4.42$

4.92

121

4.54 


\section{SHORT-TERM SALES}

YEAR: 2000 Quarter: Three

Estimated Volumes (MMCF) \& Prices (\$ / MMBTU)

Importer

Seller

Purchaser / End User

CXY ENERGY MARKETING (U.S.A.) INC.

CXY Marketing

Duke Energy Trading \& Marketing

CXY ENERGY MARKETING (U.S.A.) INC.

CXY Marketing

Duke Energy Trading \& Marketing

CXY ENERGY MARKETING (U.S.A.) INC. CXY Marketing

Enron North America Corp.

CXY ENERGY MARKETING (U.S.A.) INC. CXY Marketing

Enron North America Corp.

CXY ENERGY MARKETING (U.S.A.) INC. CXY Marketing

Equitable Resources Marketing

CXY ENERGY MARKETING (U.S.A.) INC. CXY Marketing

Interstate Power Company

CXY ENERGY MARKETING (U.S.A.) INC

CXY Marketing

Minnesota Com Processors

CXY ENERGY MARKETING (U.S.A.) INC. CXY Marketing

National Fuel Gas Distribution

CXY ENERGY MARKETING (U.S.A.) INC. CXY Marketing

National Fuel Gas Distribution

CXY ENERGY MARKETING (U.S.A.) INC. CXY Marketing

National Fuel Resources

CXY ENERGY MARKETING (U.S.A.) INC. CXY Marketing

Niagara Mohawk

CXY ENERGY MARKETING (U.S.A.) INC.

CXY Marketing

Northem Illinois Gas
Noyes, Minnesota

Point
of

Entry

Eastport, Idaho

Noyes, Minnesota

Eastport, Idaho

Niagara Falls, NY

Port of Morgan, MT

Port of Morgan, MT

Grand Island, NY

Niagara Falls, NY

Niagara Falls, NY

Niagara Falls, NY

Port of Morgan, MT
Vol. July Price

August
Vol. Price

September

Quarterly

Total

vol. ${ }^{\text {Avg. }}$ Price

$98 \quad 3.50$

$10 \quad 2.94$

$91 \quad 4.50$

199

3.93

0 N/A

$81 \quad 4.06$

- N/A

81

4.06

$30 \quad 3.69$

o N/A

$10 \quad 4.35$

40

3.86

$\begin{array}{llll}0 & \text { N/A } & 266 & 4.40\end{array}$

$0 \quad$ N/A

266

4.40

$6 \quad 4.31$

- N/A

o N/A

$6 \quad 4.31$

N N/A

$96 \quad 3.48$

- N/A

96

3.48

$98 \quad 3.27$

$173 \quad 4.31$

276

3.94

$56 \quad 4.06$

- N/A

o N/A

$56 \quad 4.06$ $\begin{array}{llll}0 & \text { N/A } & 13 \quad 458\end{array}$

$0 \quad$ N/A

$13 \quad 4.58$

- N/A

151

3.98

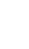

$0 \quad$ N/A

15

3.98

o N/A

$16 \quad 4.59$

- N/A

$16 \quad 4.59$

D.A 16.59

$630 \quad 3.80$

o N/A

o N/A

$630 \quad 3.80$ 


\section{SHORT-TERM SALES}

YEAR: 2000 Quarter: Three

Estimated Volumes (MMCF) \& Prices (\$ / MMBTU)

Importer

Seller
Purchaser / End User

CXY ENERGY MARKETING (U.S.A.) INC.

CXY Marketing

Northshore Gas Company

CXY ENERGY MARKETING (U.S.A.) INC.

CXY Marketing

OGE Energy Resources

CXY ENERGY MARKETING (U.S.A.) INC.

CXY Marketing

PG\&E Energy Trading

CXY ENERGY MARKETING (U.S.A.) INC.

CXY Marketing

Pancanadian Énergy Services

CXY ENERGY MARKETING (U.S.A.) INC.

CXY Marketing

Pancanadian Energy Services

CXY ENERGY MARKETING (U.S.A.) INC.

CXY Marketing

Petrocom Energy Group Ltd.

CXY ENERGY MARKETING (U.S.A.) INC.

CXY Marketing

Proliance Energy, LLC

CXY ENERGY MARKETING (U.S.A.) INC.

CXY Marketing

Reliant Energy Services, Inc.

CXY ENERGY MARKETING (U.S.A.) INC.

CXY Marketing

SCANA Energy Marketing

CXY ENERGY MARKETING (U.S.A.) INC.

CXY Marketing

Sempra Energy Trading Corp.

CXY ENERGY MARKETING (U.S.A.) INC.

CXY Marketing

Sierra Pacific Power Company

CXY ENERGY MARKETING (U.S.A.) INC.

CXY Marketing

Sigcorp Energy Services, Inc.
Point

Entry

Port of Morgan, MT

Noyes, Minnesota

Noyes, Minnesota

Noyes, Minnesota

Port of Morgan, MT

- N/A

$169 \quad 3.94$

$6 \quad 4.64$

176

3.97

Niagara Falls, NY

$198 \quad 4.16$

$112 \quad 4.67$

o N/A

310

4.34

Noyes, Minnesota

- N/A

$62 \quad 3.89$

$14 \quad 5.48$

75

4.18

Eastport, Idaho

$325 \quad 3.21$

$0 \quad$ N/A

491

3.26

Noyes, Minnesota

$\begin{array}{llll}0 & \text { N/A } & 153 & 4.35\end{array}$

$0 \quad$ N/A

153

4.35

Noyes, Minnesota

- N/A

$229 \quad 4.44$

$222 \quad 4.76$

$451 \quad 4.60$

Eastport, Idaho

$15 \quad 3.15$

13.12

$0 \quad$ N/A

17

3.15

Port of Morgan, MT $\begin{array}{llll}0 & \text { N/A } & 157 & 3.28\end{array}$

- N/A

157

3.28 


\section{SHORT-TERM SALES \\ YEAR: 2000 Quarter: Three \\ Estimated Volumes (MMCF) \& Prices ( $\$$ /MMBTU)}

Importer

Seller

Purchaser/End User

CXY ENERGY MARKETING (U.S.A.) INC.

CXY Marketing

TXU Energy Trading Company

CXY ENERGY MARKETING (U.S.A.) INC.

CXY Marketing

Tenaska Marketing Ventures

CXY ENERGY MARKETING (U.S.A.) INC.

CXY Marketing

Tenaska Washington Partners

CXY ENERGY MARKETING (U.S.A.) INC.

CXY Marketing

Texaco Natural Gas

Niagara Falls, NY

O N/A

$255 \quad 3.95$

- N/A

255

3.95

CXY ENERGY MARKETING (U.S.A.) INC. CXY Marketing

TransCanada Energy Marketing USA

Eastport, Idaho

$65 \quad 3.38$

$0 \quad$ N/A

o N/A

65

3.38

CXY ENERGY MARKETING (U.S.A.) INC. CXY Marketing

U.S. Energy Services

Port of Morgan, MT

$74 \quad 3.93$

$19 \quad 3.38$

$87 \quad 4.16$

180

3.98

CXY ENERGY MARKETING (U.S.A.) INC.

CXY Marketing

Utilicom United

Noyes, Minnesota

- N/A

$1166 \quad 3.95$

$564 \quad 4.46$

$1730 \quad 4.11$

CXY ENERGY MARKETING (U.S.A.) INC.

CXY Marketing

WPS Energy Services, Inc.

CXY ENERGY MARKETING (U.S.A.) INC

CXY Marketing

WPS Energy Services, Inc.

Port of Morgan, MT

$\begin{array}{llll}0 & \text { N/A } & 144 & 3.27\end{array}$

$0 \quad$ N/A

$144 \quad 3.27$

CXY ENERGY MARKETING (U.S.A.) INC.

CXY Marketing

Wisconsin Gas

Noyes, Minnesota

- N/A

$274 \quad 3.46$

$0 \quad$ N/A

$274 \quad 3.46$

CXY ENERGY MARKETING (U.S.A.) INC.

CXY Marketing

Wisconsin Power \& Light

Port of Morgan, MT

o N/A

$84 \quad 3.41$

$310 \quad 4.16$

$394 \quad 4.00$

DARTMOUTH POWER ASSOCIATES L.P.

Dartmouth Power Associates

Southem Company Energy
Waddington, NY
$50 \quad 3.87$

$49 \quad 4.68$

$109 \quad 5.38$

$207 \quad 4.85$ 


\section{SHORT-TERM SALES}

YEAR: 2000 Quarter: Three

Estimated Volumes (MMCF) \& Prices (\$/MMBTU)

Importer

Seller

Purchaser/End User

DEK ENERGY COMPANY

Apache Canada Ltd.

Reliant Energy Services, Inc.
Point

Entry

Eastport, Idaho
Vol. July Price

August

Vol. Price
September

Vol. Price
Quarterly

Vol. ${ }^{\text {Avg. }}$ Price

DUKE ENERGY TRADING AND MKTG SERVICES L.L.C.

Duke Energy Marketing Canada

Duke Energy Trading \& Marketing

Calais, ME

$\begin{array}{llllllll}6165 & 4.16 & 6834 & 3.78 & 6419 & 4.63 & 19418 & 4.18\end{array}$

DUKE ENERGY TRADING AND MKTG SERVICES L.L.C.

Duke Energy Marketing Canada

Duke Energy Trading \& Marketing

Eastport, Idaho

$7318 \quad 3.73$

$6695 \quad 3.30$

$6863 \quad 3.63$

$20876 \quad 3.56$

DUKE ENERGY TRADING AND MKTG SERVICES L.L.C.

Duke Energy Marketing Canada

Duke Energy Trading \& Marketing

Niagara Falls, NY

$6018 \quad 4.47$

$5948 \quad 3.95$

$5647 \quad 4.75$

17613

4.38

DUKE ENERGY TRADING AND MKTG SERVICES L.L.C.

Duke Energy Marketing Canada

Duke Energy Trading \& Marketing

Noyes, Minnesota

232

4.12

$2027 \quad 3.68$

1968

4.18

6320

4.00

DUKE ENERGY TRADING AND MKTG SERVICES L.L.C.

Duke Energy Marketing Canada

Duke Energy Trading \& Marketing

Port of Morgan, MT

$2334 \quad 3.85$

234

3.16

2254

3.98

6933

3.66

DUKE ENERGY TRADING AND MKTG SERVICES L.L.C.

Duke Energy Marketing Canada

Duke Energy Trading \& Marketing

Sumas, Washington

$2798 \quad 4.07$

$4117 \quad 3.04$

$2153 \quad 3.45$

9067

3.46

DUKE ENERGY TRADING AND MKTG SERVICES L.L.C.

Duke Energy Marketing Canada

Duke Energy Trading \& Marketing

Waddington, NY

$2950 \quad 4.53$

$1651 \quad 4.20$

6373

4.33

DYNEGY MARKETING \& TRADE

Enron North America Corp.

Dynegy Marketing \& Trade

Port of Morgan, MT

$0 \quad$ N/A

$0 \quad$ N/A

14.42

$1 \quad 4.42$

DYNEGY MARKKETING \& TRADE

Northem Natural Gas

Dynegy Marketing \& Trade

Port of Morgan, MT

$649 \quad 3.73$

578

4.52

1928

4.15

DYNEGY MARKETING \& TRADE

PanCanadian Petroleum Ltd.

Dynegy Marketing \& Trade

Port of Morgan, MT

- N/A

$28 \quad 4.45$

$0 \quad$ N/A

28

4.45

DYNEGY MARKETING \& TRADE

Sempra Energy Trading

Dynegy Marketing \& Trade

Port of Morgan, MT

- N/A

o N/A

300

4.53

300

4.53 


\section{SHORT-TERM SALES}

YEAR: 2000 Quarter: Three

Estimated Volumes (MMCF) \& Prices (\$ / MMBTU)

Importer

Seller

Purchaser / End User

DYNEGY MARKETING \& TRADE

Southem Company Energy

Dynegy Marketing \& Trade

\section{DYNEGY MARKETING \& TRADE}

Tenaska Marketing Ventures

Dynegy Marketing \& Trade

EL PASO ENERGY MARKETING COMPANY

El Paso Energy Markeing Canada

El Paso Energy Marketing

EL PASO ENERGY MARKETING COMPANY

\section{MGI Supply}

El Paso Energy Marketing

ENERGY USA-TPC CORP.

Coral Energy Canada Inc.

Various NY State Markets

ENERGY USA-TPC CORP.

El Paso Energy Markeing Canada

Various NY State Markets

ENERGY USA-TPC CORP.

El Paso Merchant Energy-Gas Company

Various NY State Markets

ENERGY USA-TPC CORP.

Enron North America Corp.

Various NY State Markets

ENERGY USA-TPC CORP

TransCanada Gas Services Limited

Various NY State Markets

Niagara Falls, NY

- N/A

$680 \quad 4.27$

$18 \quad 4.98$

698

4.29

ENGAGE ENERGY CANADA, L.P.

Engage Energy Canada

Sumas, Washington

- N/A

$18 \quad 3.08$

o N/A

18

3.08

ENGAGE ENERGY CANADA, L.P.

Engage Energy Canada

Intalco Aluminum Corporation

Sumas, Washington

$84 \quad 4.10$

$\begin{array}{ll}88 & 3.07\end{array}$

$80 \quad 3.48$

$251 \quad 3.54$

ENGAGE ENERGY CANADA, L.P.

Engage Energy Canada

Kimball Energy Corporation 


\section{SHORT-TERM SALES \\ YEAR: 2000 Quarter: Three \\ Estimated Volumes (MMCF) \& Prices (\$/MMBTU)}

Importer

Seller

Purchaser / End User

ENGAGE ENERGY CANADA, L.P.

Engage Energy Canada

Northwest Alloys

ENGAGE ENERGY CANADA, L.P.

Engage Energy Canada

Portland General Electric Company

ENGAGE ENERGY CANADA, L.P.

Engage Energy Canada

Potsdam College

ENGAGE ENERGY CANADA, L.P.

Engage Energy Canada

Puget Sound Energy

ENGAGE ENERGY CANADA, L.P.

Engage Energy Canada

SCANA Energy Marketing

ENGAGE ENERGY CANADA, L.P.

Engage Energy Canada

Southem Company Energy

ENGAGE ENERGY CANADA, L.P.

Engage Energy Canada

Tesoro Northwest Company

ENGAGE ENERGY CANADA, L.P.

Engage Energy Canada

Tosco Refining Company

ENGAGE ENERGY CANADA, L.P.

Engage Energy Canada

Various WA State Markets

ENGAGE ENERGY U.S., L.P.

Engage Energy Canada

Engage Energy U.S. LP

ENGAGE ENERGY U.S., L.P.

Engage Energy Canada

Engage Energy U.S. LP

ENGAGE ENERGY U.S., L.P.

Engage Energy Canada

Engage Energy U.S. LP
Point

Entry

Sumas, Washington

Sumas, Washington

Massena, New York

Sumas, Washington

Sumas, Washington

Sumas, Washington

Sumas, Washington

Sumas, Washington

Sumas, Washington

Eastport, Idaho

Niagara Falls, NY

Noyes, Minnesota
Vol July August

Vol. Price

September

Vol. Price

Quarterly

Total

Vol. ${ }^{\text {Avg. }}$ Price

$98 \quad 3.86$

$\begin{array}{ll}79 & 3.08\end{array}$

$85 \quad 3.48$

$263 \quad 3.50$

$301 \quad 1.73$

$303 \quad 1.70$

$291 \quad 1.70$

$895 \quad 1.71$

$16 \quad 278$

$\begin{array}{ll}16 & 2.78\end{array}$

$\begin{array}{ll}15 & 2.78\end{array}$

$47 \quad 2.78$

$29 \quad 3.25$

$0 \quad$ N/A

o N/A

$29 \quad 3.25$

$13 \quad 4.02$

$13 \quad 3.15$

$9 \quad 3.58$

35

3.59

o N/A

$10 \quad 3.01$

$0 \quad$ N/A

$10 \quad 3.01$

$151 \quad 4.09$

$164 \quad 3.06$

$147 \quad 3.47$

462

3.53

$53 \quad 3.58$

$37 \quad 3.18$

$34 \quad 4.60$

124

3.74

$559 \quad 4.07$

$630 \quad 3.04$

$513 \quad 3.45$

1702

3.50

$857 \quad 4.66$

$859 \quad 4.11$

$825 \quad 5.97$

$2540 \quad 4.90$

1554.49

- N/A

$60 \quad 5.45$

$215 \quad 4.76$

$237 \quad 3.44$

1207

3.90

2037

3.76 


\section{SHORT-TERM SALES}

YEAR: 2000 Quarter: Three

Estimated Volumes (MMCF) \& Prices (\$/MMBTU)

Importer
Seller

Purchaser / End User

ENGAGE ENERGY U.S., L.P.

Engage Energy Canada

Engage Energy U.S. LP

ENGAGE ENERGY U.S., L.P.

Engage Energy Canada

Engage Energy U.S. LP

ENRON ENERGY SERVICES, INC.

CXY Marketing

Various NY State Markets

Grand Island, NY

- N/A

O N/A

$105 \quad 4.90$

105

4.90

ENRON ENERGY SERVICES, INC.

Coral Energy Canada Inc.

Various NY State Markets

ENRON ENERGY SERVICES, INC.

Enron North America Corp.

Various NY State Markets

Grand Island, NY

Vol.
July Price August

Total

Vol. ${ }^{\text {Avg. }}$ Price

Port of Morgan, MT

$3456 \quad 4.28$

$3469 \quad 3.60$

$4028 \quad 4.55$

10953

4.16

Grand Island, NY

$465 \quad 4.60$

$414 \quad 4.20$

$305 \quad 4.95$

1184

4.55

$1193 \quad 4.65$

$434 \quad 4.20$

$406 \quad 4.90$

2033

4.60
Quarterly

ENRON INTERNATIONAL GAS SALES COMPANY

Oman LNG

Enron intemational Gas Sales Co.

Lake Charles, LA

- N/A

o N/A

$2740 \quad 3.58$

2740

3.58

ENRON NORTH AMERICA CORPORATION

B.C. Gas Inc.

Enron North America Corp.

Eastport, Idaho

$20 \quad 4.00$

O N/A

o N/A

$20 \quad 4.00$

ENRON NORTH AMERICA CORPORATION

B.C. Gas Inc.

Enron North America Corp.

Sumas, Washington

$24 \quad 3.24$

- N/A

O N/A

$24 \quad 3.24$

ENRON NORTH AMERICA CORPORATION

Enron Gas Marketing Canada

Enron North America Corp.

ENRON NORTH AMERICA CORPORATION

Enron Gas Marketing Canada

Enron North America Corp.

ENRON NORTH AMERICA CORPORATION

Enron Gas Marketing Canada

Enron North America Corp.

ENRON NORTH AMERICA CORPORATION

Enron Gas Marketing Canada

Enron North America Corp.
Eastport, Idaho

278

279

3.91

$2687 \quad 5.54$

$8266 \quad 4.63$

Grand Island, NY

$4 \quad 4.44$

$4 \quad 2.49$

$4 \quad 5.00$

$12 \quad 3.97$

Niagara Falls, NY

159

4.68

159

4.64

15

4.79

473

4.70

Port of Morgan, MT
4.17

63

3.59

639

4.23

1920

4.00 


\section{SHORT-TERM SALES \\ YEAR: 2000 Quarter: Three \\ Estimated Volumes (MMCF) \& Prices (\$ / MMBTU)}

Importer
Seller
Purchaser/ End User
ENRON NORTH AMERICA CORPORATION
Enron Gas Marketing Canada
Enron North America Corp.

ENRON NORTH AMERICA CORPORATION

Enron Gas Marketing Canada

Enron North America Corp.

H.Q. ENERGY SERVICES (U.S.) INC.

MEHQ

H.Q. Energy Services (U.S.) Inc.

HESS ENERGYINC.

CXY Marketing

Amerada Hess

HESS ENERGY INC.

Canadian Occidental Petroleum Ltd.

Amerada Hess

Grand Island, NY

- N/A

124

3.96

$0 \quad$ N/A

124

3.96

HESS ENERGY SERVICES COMPANY, LLC

Gulf Canada Resources Limited

Pancanadian Energy Services

Port of Morgan, MT

$1246 \quad 3.88$

1251

3.42

121

3.96

3713

3.75

HUSKY GAS MARKETING, INC.

Husky Oil

Barret Resources Corporation

Eastport, Idaho

$245 \quad 3.99$

o N/A

o N/A

$245 \quad 3.99$

HUSKY GAS MARKETING, INC.

Husky Oil

Caroinal IG Company

Noyes, Minnesota

$115 \quad 4.24$

$113 \quad 3.74$

111

4.40

339

4.13

HUSKY GAS MARKETING, INC.

Husky Oil

Cibola Corporation

Port of Morgan, MT

$298 \quad 3.87$

$655 \quad 3.38$

422

4.38

1375

3.79

HUSKY GAS MARKETING, INC.

Husky Oil

Dynegy Marketing \& Trade

Eastport, Idaho

$84 \quad 3.50$

$513 \quad 5.94$

473

7.28

1070

6.34

HUSKY GAS MARKETING, INC.

Husky Oil

Enron North America Corp.

Eastport, Idaho

- N/A

$0 \quad N / A$

10

7.90

$101 \quad 7.90$

HUSKY GAS MARKETING, INC.

Husky Oil

MidAmerican Energy Company 


\section{SHORT-TERM SALES \\ YEAR: 2000 Quarter: Three}

Estimated Volumes (MMCF) \& Prices (\$ / MMBTU)

Importer

Seller

Purchaser/ End User

HUSKY GAS MARKETING, INC.

Husky Oil

Montana Power

HUSKY GAS MARKETING, INC.

Husky Oil

PG\&E Energy Trading

HUSKY GAS MARKETING, INC.

Husky Oil

Pancanadian Energy Services

HUSKY GAS MARKETING, INC.

Husky Oil

Peoples Gas, Light, \& Coke

HUSKY GAS MARKETING, INC. Husky Oil

Phoenix Chemical

HUSKY GAS MARKETING, INC. Husky Oil

Sierra Pacific Power Company

HUSKY GAS MARKETING, INC. Husky Oil

Tenaska Marketing Ventures

HUSKY GAS MARKETING, INC. Husky Oil

U.S. Energy Services

IGI RESOURCES, INC.

Duke Energy Marketing Canada Avista Corporation

IGI RESOURCES, INC.

Duke Energy Marketing Canada Cascade Natural Gas

IGI RESOURCES, INC.

Duke Energy Marketing Canada Intermountain Gas Company

IGI RESOURCES, INC.

Duke Energy Marketing Canada Intermountain Gas Company
Point

Of Entry

Port of Morgan, MT

$29 \quad 3.03$

$31 \quad 2.88$

$28 \quad 4.37$

88

3.40

Port of Morgan, MT

$295 \quad 3.96$

$307 \quad 3.33$

$267 \quad 4.37$

869

3.86

Port of Morgan, MT

$131 \quad 3.69$

o N/A

$149 \quad 4.88$

$281 \quad 4.32$

Port of Morgan, MT

$744 \quad 4.04$

772

Port of Morgan, MT

$144 \quad 3.96$

153

3.33

$140 \quad 4.37$

$437 \quad 3.87$

Eastport, Idaho

- N/A

$77 \quad 5.85$

o N/A

$77 \quad 5.85$

Port of Morgan, MT

$430 \quad 3.95$

220

3.60

$374 \quad 4.70$

1024

4.15

Port of Morgan, MT

192

4.39

$178 \cdot 3.24$

$165 \quad 4.77$

535

4.12

Sumas, Washington

$21 \quad 4.10$

$36 \quad 3.07$

$60 \quad 3.48$

117

3.47

Sumas, Washington

$498 \quad 4.10$

63

3.07

$637 \quad 3.48$

1772

3.51

Eastport, Idaho

$807 \quad 3.73$

$80 \quad 3.30$

506

3.63

1394

3.67

Sumas, Washington
$645 \quad 3.07$

62 
Importer

Purchaser / End User

IGI RESOURCES, INC.

Duke Energy Marketing Canada

Northwest Natural Gas Company

IGI RESOURCES, INC.

Duke Energy Marketing Canada Puget Sound Energy

IGI RESOURCES, INC.

Duke Energy Marketing Canada Statoil Energy Services, Inc.

IGI RESOURCES, INC.

Duke Energy Marketing Canada Unocal

Sumas, Washington

Sumas, Washington

Sumas, Washington

Sumas, Washington

Sumas, Washington

$336 \quad 4.10$

$249 \quad 3.07$

$273 \quad 3.48$

$858 \quad 3.60$

IGI RESOURCES, INC.

Engage Energy Canada

Intermountain Gas Company

IGI RESOURCES, INC.

Engage Energy Canada

Northwest Natural Gas Company

IGI RESOURCES, INC.

Engage Energy Canada

Puget Sound Energy

IGI RESOURCES, INC.

Engage Energy Canada

Statoil Energy Services, inc.

July
Vol. Pugust
Price

$\begin{array}{llllllll}389 & 4.10 & 515 & 3.07 & 533 & 3.48 & 1437 & 3.50\end{array}$

$\begin{array}{llllllll}33 & 4.10 & 0 & \text { N/A } & 0 & \text { N/A } & 33 & 4.10\end{array}$

$\begin{array}{llllllll}34 & 4.10 & 117 & 3.07 & 109 & 3.48 & 260 & 3.38\end{array}$

$\begin{array}{llllllll}0 & \text { N/A } & 144 & 3.07 & 70 & 3.48 & 213 & 3.20\end{array}$

$\begin{array}{llllllll}38 & 4.10 & 161 & 3.07 & 26 & 3.48 & 225 & 3.29\end{array}$

$\begin{array}{llllllll}14 & 4.10 & 14 & 3.07 & 26 & 3.48 & 54 & 3.54\end{array}$

$\begin{array}{lllllllll}\text { Sumas, Washington } & 125 & 4.10 & 252 & 3.07 & 267 & 3.48 & 645 & 3.44\end{array}$

$\begin{array}{lllllllll}\text { Sumas, Washington } & 224 & 4.10 & 152 & 3.07 & 233 & 3.48 & 609 & 3.61\end{array}$

$\begin{array}{lllllllll}\text { Sumas, Washington } & 262 & 4.10 & 201 & 3.07 & 228 & 3.48 & 692 & 3.60\end{array}$

$\begin{array}{lllllllll}\text { Sumas, Washington } & 22 & 4.10 & 0 & \text { N/A } & 0 & \text { N/A } & 22 & 4.10\end{array}$ 


\section{SHORT-TERM SALES \\ YEAR: 2000 Quarter: Three \\ Estimated Volumes (MMCF) \& Prices (\$ / MMBTU)}

Importer
Seller

Seller
Purchaser / End User

IGI RESOURCES, INC.

Engage Energy Canada Unocal

IGI RESOURCES, INC. Engage Energy Canada Weyemauser

IGI RESOURCES, INC. Engage Energy Canada Willamette

IGI RESOURCES, INC. Poco Petroleum Ltd.

Avista Corporation

IGI RESOURCES, INC.

Poco Petroleum Ltd.

Cascade Natural Gas

IGI RESOURCES, INC.

Poco Petroleum Ltd.

Intermountain Gas Company

IGI RESOURCES, INC.

Poco Petroleum Ltd.

Northwest Natural Gas Company

IGI RESOURCES, INC.

Poco Petroleum Ltd.

Puget Sound Energy

IGI RESOURCES, INC.

Poco Petroleum Ltd.

Statoil Energy Services, Inc.

IGI RESOURCES, INC.

Poco Petroleum Ltd.

Unocal

IGI RESOURCES, INC.

Poco Petroleum Lid.

Weyerhauser

IGI RESOURCES, INC.

Poco Petroleum Lid.

Willamette
Point

Of

Vol. July Price

August
Vol. Price

September

Vol. Price

$\begin{aligned} & \text { Quarterly } \\ & \text { Total } \\ & \text { Avg. } \\ & \text { Vol. Price }\end{aligned}$

Sumas, Washington

$23 \quad 4.10$

$46 \quad 3.07$

$47 \quad 3.48$

115

3.44

Sumas, Washington

$$
\text { o N/A }
$$

$\begin{array}{ll}56 & 3.07\end{array}$

30

3.48

86

3.21

Sumas, Washington

$25 \quad 4.10$

$63 \quad 3.07$

$11 \quad 3.48$

99

3.38

Sumas, Washington

$17 \quad 4.10$

$17 \quad 3.07$

29

3.48

63

3.54

Sumas, Washington

$399 \quad 4.10$

$296 \quad 3.07$

307

3.48

1002

3.61

Sumas, Washington

$149 \quad 4.10$

300

3.07

300

3.48

749

3.44

Sumas, Washington

26

4.10

180

3.07

262

3.48

709

3.61

Sumas, Washington

3124.10

$239 \quad 3.07$

256

3.48

808

3.60

Sumas, Washington

$26 \quad 4.10$

$0 \quad$ N/A

o N/A

26

4.10

Sumas, Washington

$27 \quad 4.10$

$54 \quad 3.07$

$\begin{array}{ll}53 & 3.48\end{array}$

134

3.44

Sumas, Washington

- N/A

$67 \quad 3.07$

$34 \quad 3.48$

100

3.21

Sumas, Washington
$13 \quad 3.48$

118

3.38 


\section{SHORT-TERM SALES \\ YEAR: 2000 Quarter: Three \\ Estimated Volumes (MMCF) \& Prices (\$/MMBTU)}

Importer

Seller

Purchaser/End User

INDECK-OSWEGO, L.P.

Aquila Canada Corporation

Indeck Energy Services of Oswego

INDECK-OSWEGO, L.P.

Direct Canada

Indeck Energy Services of Oswego

INDECK-OSWEGO, L.P.

Enron Gas Marketing Canada

Indeck Energy Services of Oswego

INDECK-YERKES, L.P.

Direct Canada

Indeck Energy Services of Yerkes

INDECK-YERKES, L.P.

Northstar Energy Corporation

Indeck Energy Services of Yerkes

MONTANA-DAKOTA UTILITIES CO.

Tenaska Marketing Canada

Montana-Dakota Utilities Co.

MURPHY GAS GATHERING

Murphy Oil Company Ltd.

Murphy Gas Gathering

NATIONAL FUEL GAS DISTRIBUTION CXY Marketing

Various NY State Markets

NATIONAL FUEL GAS DISTRIBUTION Coral Energy Canada Inc.

Various NY State Markets

NATIONAL FUEL GAS DISTRIBUTION Enron North America Corp.

Various NY State Markets

NATIONAL FUEL GAS DISTRIBUTION TransCanada Gas Services Limited Various NY State Markets

NATIONAL FUEL RESOURCES, INC.

CXY Marketing

Various NY State Markets
Point

of

Vol. July

August

Vol. Price

September

Vol. Price

Quarterly

Total

Vol. ${ }^{\text {Avg. Price }}$

Niagara Falls, NY

$265 \quad 4.56$

- N/A

o N/A

265

4.56

Niagara Falls, NY

- N/A

37

4.34

36

4.72

735

4.53

Niagara Falls, NY

$109 \quad 4.19$

- N/A

- N/A

109

4.19

Niagara Falls, NY

$201 \quad 4.19$

202

4.19

196

4.72

599

4.36

Niagara Falls, NY

$172 \quad 2.67$

$172 \quad 2.77$

166

2.99

509

2.81

Port of Morgan, MT

$255 \quad 3.90$

304

286

3.80

845

3.71

Noyes, Minnesota

$101 \quad 3.93$

10

3.41

98

4.18

299

3.84

Niagara Falls, NY

$151 \quad 4.08$

$5 \quad 4.69$

$15 \quad 5.59$

171

4.23

Niagara Falls, NY

$407 \quad 4.22$

$355 \quad 4.46$

$61 \quad 5.57$

$824 \quad 4.42$

Niagara Falls, NY

$30 \quad 4.13$

$0 \quad$ N/A

$0 \quad$ N/A

30

4.13

Niagara Falls, NY

$231 \quad 4.21$

343

4.53

$38 \quad 5.61$

61

4.48

Niagara Falls, NY $\begin{array}{llll}321 & 4.48 & 150 & 3.98\end{array}$

$49 \quad 5.12$

520

4.40 


\section{SHORT-TERM SALES \\ YEAR: 2000 Quarter: Three}

Estimated Volumes (MMCF) \& Prices (\$ / MMBTU)
Importer

Seller

Purchaser / End User

NATIONAL FUEL RESOURCES, INC.

Coral Energy Canada Inc.

Various NY State Markets

NATIONAL FUEL RESOURCES, INC.

Coral Energy Canada Inc.

Various NY State Markets

NATIONAL FUEL RESOURCES, INC.

Duke Energy Marketing Canada

Various NY State Markets

NATIONAL FUEL RESOURCES, INC.

Engage Energy Canada

Vanious NY State Markets

NATIONAL FUEL RESOURCES, INC.

Indeck-Yerkes Energy Services

Various NY State Markets

NATIONAL FUEL RESOURCES, INC.

PG\&E Energy Trading

Various NY State Markets

NATIONAL FUEL RESOURCES, INC.

Renaissance Energy Ltd.

Various NY State Markets

NATIONAL FUEL RESOURCES, INC.

Renaissance Energy Ltd.

Various NY State Markets

NATIONAL FUEL RESOURCES, INC.

TXU

Various NY State Markets

Niagara Falls, NY

153

4.47

o N/A

o N/A

$153 \quad 4.47$

NATIONAL FUEL RESOURCES, INC.

Tenaska Marketing Canada

Various NY State Markets

NATIONAL FUEL RESOURCES, INC.

TransCanada Gas Services Limited

Various NY State Markets

Grand Island, NY

10. 4.31

$7 \quad 4.58$

5.44

$33 \quad 4.93$

NATIONAL FUEL RESOURCES, INC. TransCanada Gas Services Limited

Various NY State Markets

Point

Entry

Niagara Falls, NY

$15 \quad 4.48$

$15 \quad 3.95$

$0 \quad$ N/A

$31 \quad 4.22$

Niagara Falls, NY
$127 \quad 4.08$

15

4.46

182

5.26

$466 \quad 4.67$ 


\section{SHORT-TERM SALES \\ YEAR: 2000 Quarter: Three \\ Estimated Volumes (MMCF) \& Prices (\$ / MMBTU)}

Importer

Seller

Purchaser/End User

NORTH AMERICAN ENERGY, INC.

Various Suppliers

North American Energy Inc.

NORTH AMERICAN ENERGY, INC.

Various Suppliers

North American Energy lnc.

NORTHWEST NATURAL GAS COMPANY

Aquila Canada Corporation

Northwest Natural Gas Company

NORTHWEST NATURAL GAS COMPANY

Coral Energy Canada Inc.

Northwest Natural Gas Company

NORTHWEST NATURAL GAS COMPANY

Reliant Energy Services Canada, Inc.

Northwest Natural Gas Company

NUMAC ENERGY (U.S.) INC.

Numac Energy

CXY Energy Marketing

NUMAC ENERGY (U.S.) INC.

Numac Energy

Peoples Natural Gas Company

NUMAC ENERGY (U.S.) INC.

Numac Energy

Tenaska Marketing Ventures

PAN-ALBERTA GAS (U.S.), INC.

Pan-Alberta Gas Ltd.

SCANA Energy Marketing

PANCANADIAN ENERGY SERVICES, INC.

PanCanadian Petroleum Ltd.

Various CA State Markets

Eastport, Idaho

PANCANADIAN ENERGY SERVICES, INC.

PanCanadian Petroleum Ltd.

Various IL State Markets

Port of Morgan, MT

5547

Vol. July Price

August

Ef Entry

Grand Island, NY

$5 \quad 4.50$

$5 \quad 3.99$

$5 \quad 4.79$

15

4.42

Niagara Falls, NY

$172 \quad 4.08$

$215 \quad 4.89$

503

4.51

Sumas, Washington

$256 \quad 3.42$

$39 \quad 3.14$

$0 \quad$ N/A

296

3.38

Sumas, Washington

$15 \quad 3.36$

$0 \quad$ N/A

$0 \quad$ N/A

15

3.36

Sumas, Washington

$49 \quad 3.25$

o N/A

- N/A

49

3.25

Port of Morgan, MT

$157 \quad 3.94$

$157 \quad 3.47$

154

4.21

469

3.87

Port of Morgan, MT

$158 \quad 3.99$

$157 \quad 3.53$

154

4.26

469

3.92

Port of Morgan, MT

$324 \quad 3.81$

$326 \quad 3.73$

$316 \quad 4.42$

966

3.98

Port of Morgan, MT

$193 \quad 3.05$

$315 \quad 3.29$

$89 \quad 4.38$

597

3.37

$3143 \quad 3.92$

$2871 \cdot 3.85$

282

3.79

8837

3.86

Hidalgo, Texas
- N/A

- N/A

$61 \quad 4.89$

61
PEMEX

MGI 


\section{SHORT-TERM SALES \\ YEAR: 2000 Quarter: Three \\ Estimated Volumes (MMCF) \& Prices (\$ / MMBTU)}

Importer
Seller
Purchaser / End User

PETRO-CANADA HYDROCARBONS, INC.

Petro-Canada Hydrocarbons Inc.

Aquila Canada Corporation

PETRO-CANADA HYDROCARBONS, INC.

Petro-Canada Hydrocarbons Inc.

Coral Energy Resources

PETRO-CANADA HYDROCARBONS, INC.

Petro-Canada Hydrocarbons Inc.

Enron North America Corp.

PETRO-CANADA HYDROCARBONS, INC.

Petro-Canada Hydrocarbons inc.

IGI Resources

PETRO-CANADA HYDROCARBONS, INC.

Petro-Canada Hydrocarbons Inc.

Peoples Gas, Light, \& Coke

PETRO-CANADA HYDROCARBONS, INC.

Petro-Canada Hydrocarbons Inc.

Reliant Energy Services, Inc.

Port of Morgan, MT

$150 \quad 2.54$

$145 \quad 2.43$

$443 \quad 2.40$

PETRO-CANADA HYDROCARBONS, INC.

Petro-Canada Hydrocarbons Inc.

Sierra Pacific Power Company

Sumas, Washington

$148 \quad 3.44$

$150 \quad 3.18$

$143 \quad 4.56$

441

3.72

PETRO-CANADA HYDROCARBONS, INC

Petro-Canada Hydrocarbons Inc.

Tenaska Marketing Ventures

Port of Morgan, MT

$1212 \quad 4.06$

$1231 \quad 3.68$

$1187 \quad 4.36$

$3631 \quad 4.03$

PETROCOM ENERGY GROUP, LTD.

Altrade

Various CT State Markets

Niagara Falls, NY

14.03

14.62

15.03

24.43

PETROCOM ENERGY GROUP, LTD.

Altrade

Various NY State Markets

Niagara Falls, NY

$103 \quad 4.03$

$59 \quad 4.62$

$50 \quad 5.03$

212

4.43

PETROCOM ENERGY GROUP, LTD.

Altrado

Various NY State Markets

Waddington, NY

$10 \quad 3.90$

$0 \quad$ N/A

$0 \quad$ N/A

10

3.90

PETROCOM ENERGY GROUP, LTD.

Bay State Gas Company

Various NY State Markets
Niagara Falls, NY
$0 \quad$ N/A
36

4.21 
Importer

Seller

Purchaser/End User

PETROCOM ENERGY GROUP, LTD.

Brooklyn Union

Various NY State Markets

Niagara Falls, NY

$0 \quad$ N/A

$16 \quad 4.41$

$0 \quad$ N/A

$16 \quad 4.41$

PETROCOM ENERGY GROUP, LTD.

CXY Marketing

Various CT State Markets

Niagara Falls, NY

$2 \quad 4.17$

14.68

$0 \quad 4.98$

$3 \quad 4.36$

PETROCOM ENERGY GROUP, LTD.

CXY Marketing

Various CT State Markets

Waddington, NY

14.25

$0 \quad 4.69$

$0 \quad$ N/A

14.33

PETROCOM ENERGY GROUP, LTD.

CXY Marketing

Various NY State Markets

Niagara Falls, NY

201

4.17

110

4.68

$92 \quad 4.25$

$21 \quad 4.69$

$0 \quad$ N/A

113

4.33

PETROCOM ENERGY GROUP, LTD.

Coral Energy Canada Inc.

Various CT State Markets

PETROCOM ENERGY GROUP, LTD.

Coral Energy Canada Inc.

Various NY State Markets

PETROCOM ENERGY GROUP, LTD.

Coral Energy Canada Inc.

Various NY State Markets

PETROCOM ENERGY GROUP, LTD. Enron Gas Marketing Canada

Various CT State Markets

Niagara Falls, NY

$1 \quad 4.12$

$0 \quad 4.46$

$0 \quad$ N/A

$2 \quad 4.19$

PETROCOM ENERGY GROUP, LTD, Enron Gas Marketing Canada

Various NY State Markets

Niagara Falls, NY

126

4.12

$32 \quad 4.46$

$0 \quad$ N/A

158

4.19

PETROCOM ENERGY GROUP, LTD.

Enron Gas Marketing Canada

Various NY State Markets

Waddington, NY

$25 \quad 4.41$

$0 \quad$ N/A

$0 \quad$ N/A

25

4.41

PETROCOM ENERGY GROUP, LTD.

TransCanada Gas Services Limited

Various CT State Markets

Niagara Falls, NY
$1 \quad 4.21$

$0 \quad$ N/A

$0 \quad 5.38$

14.61 


\section{SHORT-TERM SALES \\ YEAR: 2000 Quarter: Three \\ Estimated Volumes (MMCF) \& Prices (\$ / MMBTU)}

Importer

Seller

Purchaser / End User

PETROCOM ENERGY GROUP, LTD.

TransCanada Gas Services Limited

Various NY State Markets

PETROCOM ENERGY GROUP, LTD.

TransCanada Gas Services Limited

Various NY State Markets

PETROCOM ENERGY GROUP, LTD.

TransCanada Pipelines Limited

Various CT State Markets

PETROCOM ENERGY GROUP, LTD.

TransCanada Pipelines Limited

Various NY State Markets

Niagara Falls, NY

Vol. July Price

August

of

Entry

Niagara Falls, NY

$\begin{array}{ll}58 & 4.21\end{array}$

$0 \quad$ N/A

$31 \quad 5.38$

89

4.61

Waddington, NY

$6 \quad 4.14$

$0 \quad$ N/A

$6 \quad 5.33$

12

4.72

Niagara Falls, NY

O N/A

14.66

O N/A

14.66

PETROCOM ENERGY GROUP, LTD.

TransCanada Pipelines Limited

Various NY State Markets

Waddington, NY

o N/A

$7 \quad 4.67$

$0 \quad$ N/A

$7 \quad 4.67$

PG\&E CORE PROCUREMENT DEPT

Amoco Canada Petroleum Company Litd. PG\&E Core Procurement Dept.

Eastport, Idaho

$720 \quad 3.32$

$621 \quad 3.22$

1920

3.42

PG\&E CORE PROCUREMENT DEPT.

Aquila Canada Corporation

PG\&E Core Procurement Dept.

Eastport, Idaho

Eastport, Idaho

Eastport, Idaho

2528

Eastport, Idaho

8285

794

3.31

$8559 \quad 3.27$

24790

3.43

PG\&E CORE PROCUREMENT DEPT.

Gulf Midstream Services

PG\&E Core Procurement Dept.

PG\&E CORE PROCUREMENT DEPT.

$J$. Aron \& Company

PG\&E Core Procurement Dept.
Eastport, Idaho

145

3.76

144

3.32

155

3.22

444

3.43

Eastport, Idaho
3.74

1332

3.32

143

3.28

4097

3.44 
SHORT-TERM SALES

YEAR: 2000 Quarter: Three

Estimated Volumes (MMCF) \& Prices (\$ / MMBTU)
Importer

Seller

Purchaser / End User

PG\&E CORE PROCUREMENT DEPT.

Natural Gas Exchange

PG\&E Core Procurement Dept.

PG\&E CORE PROCUREMENT DEPT.

Reliant Energy Services Canada, Inc.

PG\&E Core Procurement Dept.

PG\&E CORE PROCUREMENT DEPT.

Sempra Energy Trading

PG\&E Core Procurement Dept.

PG\&E CORE PROCUREMENT DEPT.

TXU

PG\&E Core Procurement Dept.

Eastport, Idaho

Point

Entry

Eastport, Idaho

Eastport, Idaho

Eastport, Idaho

Port of Morgan, MT

Noyes, Minnesota

Port of Morgan, MT

Eastport, Idaho

Port of Morgan, MT

Noyes, Minnesota

O N/A

$3 \quad 4.72$

O N/A

$3 \quad 4.72$

POCO MARKETING LTD.

Poco Marketing Ltd.

Pancanadian Energy Services

Port of Morgan, MT

POCO MARKETING LTD.

Poco Marketing Ltd.

Puget Sound Energy

Vol.
July Price

Quarterly
Total

Vol. Avg. Price

$\begin{array}{llllllll}685 & 3.72 & 109 & 3.08 & 398 & 3.66 & 1192 & 3.64\end{array}$

$\begin{array}{llllllll}145 & 3.72 & 144 & 3.28 & 155 & 3.19 & 444 & 3.39\end{array}$

$\begin{array}{llllllll}2461 & 3.84 & 2449 & 3.35 & 2638 & 3.47 & 7547 & 3.55\end{array}$

$\begin{array}{llllllll}651 & 3.75 & 936 & 3.29 & 698 & 3.22 & 2286 & 3.40\end{array}$

$\begin{array}{llllllll}399 & 3.67 & 528 & 3.36 & 451 & 4.00 & 1378 & 3.66\end{array}$

$\begin{array}{llllllll}83 & 3.95 & 81 & 4.44 & 81 & 4.40 & 244 & 4.26\end{array}$

$\begin{array}{lllllllll}0 & N / A & - & 46 & 3.86 & 98 & 4.59 & 144 & 4.36\end{array}$

$\begin{array}{llllllll}140 & 3.98 & 140 & 3.59 & 136 & 3.96 & 416 & 3.84\end{array}$

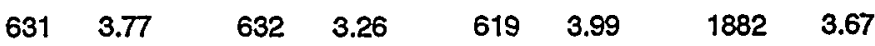

$\begin{array}{llllllll}214 & 3.67 & 8 & 3.82 & 26 & 4.16 & 248 & 3.73\end{array}$

$\begin{array}{lllllllll}\text { Sumas, Washington } & 468 & 3.55 & 441 & 3.07 & 330 & 3.48 & 1240 & 3.36\end{array}$ 
SHORT-TERM SALES -

YEAR: 2000 Quarter: Three

Estimated Volumes (MMCF) \& Prices (\$ / MMBTU)

Importer .

Seller

Purchaser/ End User

POCO MARKETING LTD.

Poco Marketing Ltd.

Quick Trade

POCO MARKETING LTD.

Poco Marketing Ltd.

POCO MARKETING LTD.

Poco Marketing Ltd.

Southem Indiana Gas

POCO MARKETING LTD.

Poco Marketing Ltd.

Tenaska Marketing Ventures
Sempra Energy Trading Corp.

Point

of

Port of Morgan, MT

Niagara Falls, NY

Port of Morgan, MT

320

3.66

236

3.23

338

3.67

$510 \quad 3.47$

$387 \quad 4.30$

1234

3.78

PORTLAND GENERAL ELECTRIC COMPANY Aquila Canada Corporation

Portland General Electric Company

Eastport, Idaho

588

588

2.34

450

1.96

$582 \quad 2.32$

579

Eastport, Idaho

Duke Energy Marketing Canada

Portland General Electric Company

PORTLAND GENERAL ELECTRIC COMPANY

Dynegy Canada

Portand General Electric Company

Eastport, Idaho

$11 \quad 3.01$

16

3.16

$140 \quad 3.50$

167

3.43

PORTLAND GENERAL ELECTRIC COMPANY

Engage Energy Canada

Portland General Electric Company

Eastport, Idaho

$311 \quad 3.66$

$440 \quad 2.46$

$\begin{array}{ll}478 & 2.68\end{array}$

1229

2.85

PORTLAND GENERAL ELECTRIC COMPANY

Sempra Energy Trading

Portland General Electric Company

Eastport, Idaho

$146 \quad 3.54$

- N/A

- N/A

146

3.54

PRAIRIELANDS ENERGY MARKETING, INC.

Canadian Blackhawk Energy, Inc.

Energy West Resources

Babb, Montana

$5 \quad 3.68$

$5 \quad 3.25$

$5 \quad 3.53$

14

3.49

PRAIRIELANDS ENERGY MARKETING, INC.

Jeffoco Holdings, Inc.

Energy West Resources

Babb, Montana

$8 \quad 3.64$

$8 \quad 3.23$

$7 \quad 3.58$

23

3.49

PRAIRIELANDS ENERGY MARKETING, INC. Primrose Drilling Ventures, Ltd.

Energy West Resources

Babb, Montana
$6 \quad 3.68$

$6 \quad 3.25$

$6 \quad 3.53$

17

3.49 


\section{SHORT-TERM SALES \\ YEAR: 2000 Quarter: Three \\ Estimated Volumes (MMCF) \& Prices (\$ / MMBTU)}

Importer

Seller
Purchaser / End User

PROGAS U.S.A., INC.

ProGas Limited

CXY Energy Marketing

PROGAS U.S.A., INC

ProGas Limited

Carthage Energy Services

PROGAS U.S.A., INC.

ProGas Limited

Coral Energy Resources

PROGAS U.S.A., INC.

ProGas Limited

Duke Energy Trading \& Marketing

PROGAS U.S.A., INC.

ProGas Limited

Dynegy Marketing \& Trade

PROGAS U.S.A., INC.

ProGas Limited

Energy Masters Intemational, Inc.

PROGAS U.S.A., INC.

ProGas Limited

Energy Masters Intemational, Inc.

PROGAS U.S.A., INC.

ProGas Limited

Energy USA

PROGAS U.S.A., INC.

ProGas Limited

Engage Energy U.S. LP

PROGAS U.S.A., INC.

ProGas Limited

Honeymead Products Company

Port of Morgan, MT

PROGAS U.S.A., INC.

ProGas Limited

Midland Cogeneration Venture

PROGAS U.S.A., INC.

ProGas Limited

New Jersey Natural Gas

Point

Ef Entry

Noyes, Minnesota

Noyes, Minnesota

Niagara Falls, NY

Noyes, Minnesota

Port of Morgan, MT

Port of Morgan, MT

Niagara Falls, NY

$306 \quad 4.56$

Noyes, Minnesota

Niagara Falls, NY
$308 \quad 4.04$
$31 \quad 3.89$

$31 \quad 3.76$

$30 \quad 4.54$

$92 \quad 4.06$

$77 \quad 4.50$

$77 \quad 3.94$

$74 \quad 4.78$

227

4.40

$87 \quad 3.89$

- N/A

- N/A

$87 \quad 3.89$

$129 \quad 4.56$

$129 \quad 4.04$

12.

4.90

$382 \quad 4.50$

$184 \quad 4.50$

184

3.94

178

4.78

$546 \quad 4.40$

Quarterly
Total

Vol. ${ }^{\text {Avg. }}$ Price

$663 \quad 4.63$

$26 \quad 4.37$

$10 \quad 4.90$

$19 \quad 4.62$

14.33

$432 \quad 4.41$

$308 \quad 4.56$

$0 \quad N / A$

$617 \quad 4.30$ 


\section{SHORT-TERM SALES \\ YEAR: 2000 Quarter: Three \\ Estimated Volumes (MMCF) \& Prices (\$ / MMBTU)}

Importer

Seller

Purchaser/End User

PROGAS U.S.A., INC.

ProGas Limited

PG\&E Energy Trading

PROGAS U.S.A., INC.

ProGas Limited

PG\&E Energy Trading

PROGAS U.S.A., INC.

ProGas Limited

Southem Company Energy

PROGAS U.S.A., INC.

ProGas Limited

TXU Energy Trading Company

PROGAS U.S.A., INC.

ProGas Limited

Tenaska Marketing Ventures

PROGAS U.S.A., INC.

ProGas Limited

TransCanada Energy Marketing USA

PROGAS U.S.A., INC.

ProGas Limited

WPS Energy Services, Inc.

PUGET SOUND ENERGY, INC.

Puget Sound Energy

Puget Sound Energy

PUGET SOUND ENERGY, INC.

Talisman LT

Puget Sound Energy

RELIANT ENERGY SERVICES, INC.

Reliant Energy Services Canada, Inc.

Reliant Energy Services, Inc.

RELIANT ENERGY SERVICES, INC.

Reliant Energy Services Canada, Inc.

Reliant Energy Services, Inc.

RENAISSANCE ENERGY (U.S.), INC.

Renaissance Energy Ltd.

Renaissance Energy (U.S.) Inc.
Point

Entry

Vol. July Price ${ }^{\text {August }}$ Price

September
Vol. Price

Quarterly

Total

Vol. Prg. Price

Niagara Falls, NY

$152 \quad 4.56$

$0 \quad$ N/A

$0 \quad$ N/A

$152 \quad 4.56$

Port of Morgan, MT

- N/A

$152 \quad 3.76$

$147 \quad 4.54$

298

4.14

Niagara Falls, NY

$126 \quad 4.56$

$306 \quad 4.04$

o N/A

432

4.19

Noyes, Minnesota

$153 \quad 4.50$

o N/A

193

4.78

347

4.66

Port of Morgan, MT

$131 \quad 3.89$

$114 \quad 3.76$

o N/A

$245 \quad 3.83$

Port of Morgan, MT

$152 \quad 3.76$

$257 \quad 4.54$

$561 \quad 4.15$

Port of Morgan, MT

$\begin{array}{llll}0 & \text { N/A } & 306 & 3.76\end{array}$

$297 \quad 4.54$

$603 \quad 4.14$

Sumas, Washington

$1742 \quad 4.10$

$3594 \quad 3.07$

$3841 \quad 3.47$

$9177 \quad 3.43$

Sumas, Washington

$170 \quad 4.11$

$170 \quad 3.08$

$300 \quad 3.49$

$640 \quad 3.55$

Eastport, Idaho

794360

$428 \quad 3.52$

$648 \quad 4.87$

$1870 \quad 4.02$

Sumas, Washington

$\begin{array}{lll}325 & 3.64 & 470 \quad 3.38\end{array}$

$223 \quad 4.69$

1018

3.75

Grand Island, NY

$45 \quad 3.19$

$49 \quad 3.19$

$47 \quad 3.10$

141

3.16 


\section{SHORT-TERM SALES}

YEAR: 2000 Quarter: Three

Estimated Volumes (MMCF) \& Prices (\$ / MMBTU)

Importer

Seller

Purchaser/End User

RENAISSANCE ENERGY (U.S.), INC.

Renaissance Energy Ltd.

Renaissance Energy (U.S.) Inc.

RENAISSANCE ENERGY (U.S.), INC.

Renaissance Energy Ltd.

Renaissance Energy (U.S.) Inc.

RENAISSANCE ENERGY (U.S.), INC.

Renaissance Energy Ltd.

Renaissance Energy (U.S.) Inc.

RENAISSANCE ENERGY (U.S.), INC.

Renaissance Energy Ltd.

Renaissance Energy (U.S.) Inc.

RENAISSANCE ENNERGY (U.S.), INC.

Renaissance Energy Ltd.

Renaissance Energy (U.S.) Inc.

Port of Morgan, MT

$2063 \quad 3.84$

2069

August

of

Havre; Montana

Vol. July Price

Vol. Price

$600 \quad 3.13$

247

3.19

$150 \quad 4.42$

997

3.34

Niagara Falls, NY

$47 \quad 4.08$

- N/A

$0 \quad$ N/A

$47 \quad 4.08$

Noyes, Minnesota

2208

226

4.05

248

3.77

234

5.28

707

4.36

RENAISSANCE ENERGY (U.S.), INC.

Renaissance Energy $L$ td.

Renaissance Energy (U.S.) Inc.

Waddington, NY

$43 \quad 4.00$

$79 \quad 4.49$

- N/A

122

4.32

ROCHESTER GAS \& ELECTRIC CORPORATION

Dynegy Canada

Rochester Gas \& Electric Company

Grand Island, NY

$952 \quad 4.29$

$967 \quad 4.28$

608

5.10

2526

4.48

SACRAMENTO MUNICIPAL UTILITY DISTRICT

Amoco Canada Marketing Corporation

Sacramento Municipal Utility Dist.

Eastport, Idaho

$0 \quad$ N/A

$\begin{array}{ll}78 & 3.40\end{array}$

$0^{\circ} \quad$ N/A

78

3.40

SACRAMENTO MUNICIPAL UTILITY DISTRICT

Amoco Canada Petroleum Company Ltd.

Sacramento Municipal Utility Dist.

Eastport, Idaho

$32 \quad 3.41$

O N/A

- N/A

32

3.41

SACRAMENTO MUNICIPAL UTILITY DISTRICT

Aquila Canada Corporation

Sacramento Municipal Utility Dist.

Eastport, Idaho

$80 \quad 3.44$

$105 \quad 3.37$

$86 \quad 4.46$

271

3.74

SACRAMENTO MUNICIPAL UTILITY DISTRICT

BP Resources Canada Limited

Sacramento Municipal Utility Dist.

Eastport, Idaho

$0 \quad$ N/A

$0 \quad$ N/A

$12 \quad 4.79$

12

4.79

SACRAMENTO MUNICIPAL UTILITY DISTRICT

Duke Energy Marketing Canada

Sacramento Municipal Utility Dist.

Eastport, Idaho
$0 \quad$ N/A

$0 \quad$ N/A

$212 \quad 4.64$

$212 \quad 4.64$ 


\section{SHORT-TERM SALES}

YEAR: 2000 Quarter: Three

Estimated Volumes (MMCF) \& Prices (\$/MMBTU)

$\begin{array}{ll}\text { Importer } & \text { Point } \\ \text { Seller } & \text { of }\end{array}$

Purchaser / End User

Entry

Eastport, Idaho

138

3.45

O N/A

$0 \quad N / A$

138

3.45

SACRAMENTO MUNICIPAL UTILITY DISTRICT

Dynegy Marketing \& Trade

Sacramento Municipal Utility Dist.

Eastport, Idaho

$0 \quad$ N/A

171

3.37

O N/A

171

3.37

SACRAMENTO MUNICIPAL UTILITY DISTRICT

Reliant Energy Services Canada, Inc.

Sacramento Municipal Utility Dist.

Eastport, Idaho

$134 \quad 3.50$

$30 \quad 3.48$

$61 \quad 4.69$

226

3.82

SAN DIEGO GAS \& ELECTRIC

B.C. Gas Inc.

San Diego Gas \& Electric Company

Eastport, Idaho

$0 \quad N / A$

Eastport, Idaho

266

3.61

Eastport, Idaho

- N/A

- N/A

$42 \quad 3.62$

$42 \quad 3.62$

SAN DIEGO GAS \& ELECTRIC

Various Suppliers

San Diego Gas \& Electric Company

Eastport, Idaho

$488 \quad 3.65$

$0 \quad$ N/A

o N/A

$488 \quad 3.65$

SEMPRA ENERGY TRADING CORPORATION

Gas Natural intemational LTD

Various LA State Markets

Lake Charies, LA

$3677 \quad 3.88$

- N/A

$0 \quad$ N/A

$3677 \quad 3.88$

SEMPRA ENERGY TRADING CORPORATION

Various Suppliers

Various CA State Markets

Eastport, Idaho

$0 \quad N / A$

145

3.50

$130 \quad \cdot 3.60$

$275 \quad 3.55$

SEMPRA ENERGY TRADING CORPORATION

Various Suppliers

Various CA State Markets

Sumas, Washington

- N/A

135

3.65

$19 \quad 3.70$

154

3.66

SEMPRA ENERGY TRADING CORPORATION

Various Suppliers

Various ME State Markets

\section{Calais, ME}

381

4.07

$4315 \quad 4.42$

3891

5.17

12023

4.55

SEMPRA ENERGY TRADING CORPORATION

Various Suppliers

Various NY State Markets
Niagara Falls, NY
$709 \quad 4.55$ 


\section{. SHORT-TERM SALES \\ YEAR: 2000 Quarter: Three \\ Estimated Volumes (MMCF) \& Prices (\$ / MMBTU)}

Importer
Seller
Purchaser / End User
SEMPRA ENERGY TRADING CORPORATION
Various Suppliers
Various WI State Markets

SIERRA PACIFIC POWER COMPANY

Amoco Canada Petroleum Company Lid.

Sierra Pacific Power Company

SIERRA PACIFIC POWER COMPANY

B.C. Gas Inc.

Sierra Pacific Power Company

SIERRA PACIFIC POWER COMPANY BP Canada Energy Marketing Corp.

Sierra Pacific Power Company

SIERRA PACIFIC POWER COMPANY Engage Energy Canada

Sierra Pacific Power Company

SPRAGUE ENERGY CORPORATION Boston Gas Company

Various MA State Markets

SPRAGUE ENERGY CORPORATION Boston Gas Company

Various MA State Markets

SPRAGUE ENERGY CORPORATION Coral Energy Canada Inc.

Various NY State Markets

SPRAGUE ENERGY CORPORATION El Paso Energy Markeing Canada Milford Power, L.P.

SPRAGUE ENERGY CORPORATION El Paso Energy Markeing Canada

Various NY State Markets

Grand Island, NY

O N/A

24.10

- N/A

$2 \quad 4.10$

SPRAGUE ENERGY CORPORATION

Renaissance Energy Ltd.

Various MA State Markets

SPRAGUE ENERGY CORPORATION

Renaissance Energy Ltd.

Various NY State Markets

Point

Entry

Eastport, Idaho

Eastport, Idaho

Eastport, Idaho

Eastport, Idaho

Niagara Falls, NY

Waddington, NY

Waddington, NY

Niagara Falls, NY

$186 \quad 4.65$

- N/A

0 N/A

186

4.65

Niagara Falls, NY

$64 \quad 4.45$

$27 \quad 3.94$

- N/A

$91 \quad 4.30$

Grand Island, NY
$96 \quad 4.48$

$97 \quad 4.76$

$76 \quad 5.45$

$269 \quad 4.85$ 


\section{SHORT-TERM SALES}

YEAR: 2000 Quarter: Three

Estimated Volumes (MMCF) \& Prices (\$/MMBTU)

Importer

Seller

Purchaser / End User

SPRAGUE ENERGY CORPORATION

Renaissance Energy Ltd.

Various NY State Markets

SPRAGUE ENERGY CORPORATION

Renaissance Energy Ltd.

Various NY State Markets

SPRAGUE ENERGY CORPORATION

TransCanada Gas Services Limited

Various MA State Markets

ST. LAWRENCE GAS COMPANY, INC.

Enron North America Corp.

Various NY State Markets

ST. LAWRENCE GAS COMPANY, INC.

TransCanada Gas Services Limited

Various NY State Markets

STAMPEDER ENERGY (U.S.) INC.

Gulf Canada Resources Limited

Avista Energy

STAMPEDER ENERGY (U.S.) INC.

Gulf Canada Resources Limited

Coast Energy Group

STAMPEDER ENERGY (U.S.) INC.

Gulf Canada Resources Limited

Hesse Gas Company

Massena, New York

Eastport, Idaho

$151 \quad 3.76$

151

3.14

145

3.73

448

3.54

Eastport, Idaho

$136 \quad 3.76$

151

3.14

134

3.73

421

3.53

- Port of Morgan, MT

122

3.52

1229

3.07

119

3.59

3642

3.39

SUNCOR ENERGY INC.

Suncor Energy lnc.

Aquila Energy Marketing

Eastport, Idaho

$228 \quad 3.88$

75

3.74

141

5.11

444

4.25

SUNCOR ENERGY INC.

Suncor Energy Inc.

Engage Energy U.S. LP

Eastport, Idaho

$233 \quad 3.88$

226

3.74

75

5.11

533

3.99

SUNCOR ENERGY INC.

Suncor Energy Inc.

Enron North America Corp.

Eastport, Idaho

- N/A

- N/A

132

5.11

132

5.11

SUNCOR ENERGY INC.

Suncor Energy inc.

Pancanadian Energy Services
Eastport, Idaho o N/A

143

5.11

143

5.11 


\section{SHORT-TERM SALES}

YEAR: 2000 Quarter: Three Estimated Volumes (MMCF) \& Prices (\$ / MMBTU)

Importer

Seller

Purchaser / End User

SUNCOR ENERGY INC.

Suncor Energy Inc.

Pancanadian Energy Services

SUNCOR ENERGY INC.

Suncor Energy Inc.

Reliant Energy Services, Inc.

SUNCOR ENERGY INC.

Suncor Energy Inc.

Southem Company Energy

SUNCOR ENERGY INC.

Suncor Energy inc.

Tenaska Marketing Ventures

SUNCOR ENERGY INC.

Suncor Energy Inc.

Wisconsin Power \& Light

TENASKA MARKETING VENTURES

Tenaska Marketing Canada

Various IA State Markets

TENASKA MARKETING VENTURES

Tenaska Marketing Canada

Various MN State Markets

Noyes, Minnesota

TEXACO ENERGY MARKETING L.P.

Various Suppliers

Texaco Energy Marketing

Niagara Falls, NY

THE MEAD CORPORATION

Coral Energy Canada Inc.

Mead Paper

Pittsburg, NH

THE MONTANA POWER TRADING \& MARKETING COMPANY

The Montana Power Trading \& Marketing Co.

Various CA State Markets

Eastport, Idaho

THE MONTANA POWER TRADING \& MARKETING COMPANY The Montana Power Trading \& Marketing Co.

Various CO State Markets

Port of Del Bonita,

THE MONTANA POWER TRADING \& MARKETING COMPANY The Montana Power Trading \& Marketing Co.

Various CO State Markets
July
Vol. Pugust
Price September

$\begin{array}{llllll}285 & 3.77 & 288 & 3.47 & 278 & 4.34\end{array}$

$\begin{array}{llllllll}784 & 3.88 & 764 & 3.74 & 688 & 5.11 & 2236 & 4.21\end{array}$

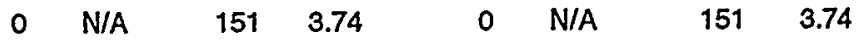

$\begin{array}{llllllll}154 & 3.77 & 155 & 3.47 & 150 & 4.34 & 459 & 3.86\end{array}$

$\begin{array}{llllllll}29 & 3.77 & 29 & 3.47 & 28 & 4.34 & 86 & 3.85\end{array}$

$\begin{array}{llllllll}1785 & 4.08 & 1798 & 3.69 & 1814 & 4.10 & 5396 & 3.96\end{array}$

$\begin{array}{llllllll}205 & 4.05 & 715 & 3.90 & 205 & 4.48 & 1125 & 4.03\end{array}$

$\begin{array}{llllllll}296 & 4.46 & 460 & 3.93 & 450 & 4.73 & 1206 & 4.36\end{array}$

$\begin{array}{llllllll}104 & 4.53 & 118 & 3.99 & 150 & 4.82 & 372 & 4.48\end{array}$

$\begin{array}{llllllll}315 & 3.41 & 315 & 3.38 & 302 & 4.66 & 932 & 3.80\end{array}$

$\begin{array}{llllllll}10 & 4.05 & 1 & 4.04 & 10 & 5.60 & 22 & 4.78\end{array}$

$\begin{array}{lllllllll}\text { Whitlash, Montana } & 1037 & 3.13 & 1005 & 3.10 & 1060 & 4.13 & 3102 & 3.46\end{array}$ 


\section{SHORT-TERM SALES}

YEAR: 2000 Quarter: Three

Estimated Volumes (MMCF) \& Prices (\$ / MMBTU)

\begin{tabular}{ll} 
Importer & Point \\
Seller & of \\
Purchaser / End User & Entry \\
\hline
\end{tabular}

THE MONTANA POWER TRADING \& MARKETING COMPANY The Montana Power Trading \& Marketing Co.

Various IL State Markets

Port of Morgan, MT

Waddington, NY

Noyes, Minnesota

$31 \quad 3.81$

$27 \quad 3.65$

$59 \quad 4.83$

117

4.28

TRANSCANADA GAS SERVICES INC.

TransCanada Gas Services Limited

Amoco Trading

Eastport, Idaho

$43 \quad 3.77$

$0 \quad$ N/A

$0 \quad$ N/A

43

3.77

TRANSCANADA GAS SERVICES INC.

TransCanada Gas Services Limited

Aquila Energy Marketing

Noyes, Minnesota

165

3.81

$151 \quad 3.65$

$173 \quad 4.83$

489

4.12

TRANSCANADA GAS SERVICES INC. TransCanada Gas Services Limited Avista Energy

Sumas, Washington

24

TRANSCANADA GAS SERVICES INC.

TransCanada Gas Services Limited

BP Energy Marketing Corp.

Eastport, Idaho

$0 \quad$ N/A

o N/A

$74 \quad 4.96$

$74 \quad 4.96$

TRANSCANADA GAS SERVICES INC.

TransCanada Gas Services Limited

BP Energy Marketing Corp.

Noyes, Minnesota

0 N/A

179

3.65

$2 \quad 4.83$

181

3.66

TRANSCANADA GAS SERVICES INC.

TransCanada Gas Services Limited

Barret Resources Corporation

Eastport, Idaho

118

TRANSCANADA GAS SERVICES INC.

TransCanada Gas Services Limited

CMS Gas Marketing

TRANSCANADA GAS SERVICES INC.

- TransCanada Gas Services Limited

Cabot Oil

Noyes, Minnesota

$1 \quad 3.81$

13.65

14.83

$2 \quad 3.98$

TRANSCANADA GAS SERVICES INC.

TransCanada Gas Services Limited

Central Hudson Gas \& Electric
Waddington, NY
$14 \quad 4.64$

$5 \quad 5.44$

30

4.50 


\section{SHORT-TERM SALES \\ YEAR: 2000 Quarter: Three \\ Estimated Volumes (MMCF) \& Prices (\$ / MMBTU)}

Importer

Seller

Purchaser/End User

TRANSCANADA GAS SERVICES INC.

TransCanada Gas Services Limited

Cibola Corporation

TRANSCANADA GAS SERVICES INC.

TransCanada Gas Services Limited

Cibola Corporation

TRANSCANADA GAS SERVICES INC.

TransCanada Gas Services Limited

Clinton Gas Marketing

TRANSCANADA GAS.SERVICES INC.

TransCanada Gas Services Limited

CoEnergy Trading Company

Noyes, Minnesota

Niagara Falls, NY

Waddington, NY

$33 \quad 3.88$

O N/A

- N/A

$33 \quad 3.88$

TRANSCANADA GAS SERVICES INC. TransCanada Gas Services Limited

Consumers Energy Company

Noyes, Minnesota

1199

1162

772

4.83

$3133 \quad 4.00$

TRANSCANADA GAS SERVICES INC.

TransCanada Gas Services Limited

Coral Energy Resources

Eastport, Idaho

192

3.77

$248 \quad 3.27$

178

4.96

618

3.91

TRANSCANADA GAS SERVICES INC. TransCanada Gas Services Limited

Coral Energy Resources

Noyes, Minnesota

$0 \quad$ N/A

O N/A

$12 \quad 4.83$

12

4.83

TRANSCANADA GAS SERVICES INC. TransCanada Gas Services Limited Coral Energy Resources

Sumas, Washington

$95 \quad 3.49$

$880 \quad 3.34$

$514 \quad 4.66$

1489

3.80

TRANSCANADA GAS SERVICES INC. TransCanada Gas Services Limited

Detroit Edison Company

TRANSCANADA GAS SERVICES INC. TransCanada Gas Services Limited

Duke Energy Trading \& Marketing
Noyes, Minnesota

Eastport, Idaho
$1031 \quad 3.81$

$323 \quad 3.65$

$162 \quad 4.83$

1516

3.88

$71 \quad 3.77$

$\begin{array}{ll}55 & 3.27\end{array}$

$61 \quad 4.96$
$187 \quad 4.01$ 


\section{SHORT-TERM SALES \\ YEAR: 2000 Quarter: Three \\ Estimated Volumes (MMCF) \& Prices (\$ / MMBTU)}

Importer

Seller

Purchaser / End User

TRANSCANADA GAS SERVICES INC.

TransCanada Gas Services Limited

Duke Energy Trading \& Marketing

TRANSCANADA GAS SERVICES INC.

TransCanada Gas Services Limited

Duke Energy Trading \& Marketing

TRANSCANADA GAS SERVICES INC.

TransCanada Gas Services Limited

Dynegy Marketing \& Trade

TRANSCANADA GAS SERVICES INC.

TransCanada Gas Services Limited

Engage Energy U.S. LP

TRANSCANADA GAS SERVICES INC.

TransCanada Gas Services Limited

Enron North America Corp.

TRANSCANADA GAS SERVICES INC.

TransCanada Gas Services Limited

Enron North America Corp.

TRANSCANADA GAS SERVICES INC.

TransCanada Gas Services Limited

Enron North America Corp.

TRANSCANADA GAS SERVICES INC.

TransCanada Gas Services Limited

Enserco Energy, Inc.

TRANSCANADA GAS SERVICES INC.

TransCanada Gas Services Limited

Fuel Imbalance

Eastport, Idaho

$22 \quad 3.77$

$19 \quad 3.27$

$19 \quad 4.96$

60

3.99

TRANSCANADA GAS SERVICES INC. TransCanada Gas Services Limited Fuel Imbalance

Noyes, Minnesota

$263 \quad 3.81$

$254 \quad 3.69$

$211 \quad 4.83$

$728 \quad 4.06$

TRANSCANADA GAS SERVICES INC. TransCanada Gas Services Limited Fuel Imbalance

Waddington, NY

$15 \quad 3.88$

$15 \quad 4.64$

$2 \quad 5.44$

32

4.33

TRANSCANADA GAS SERVICES INC.

TransCanada Gas Services Limited

IDA Corporation Energy Solutions L.P.

$500 \quad 4.02$

$17 \quad 5.44$

$144 \quad 3.83$

$\begin{array}{ll}3 & 3.77\end{array}$

$545 \quad 3.87$

$16 \quad 3.49$

$662 \quad 3.97$ o N/A

$16 \quad 3.34$

O N/A

$\begin{aligned} & \text { Quarterly } \\ & \text { Total } \\ & \text { Avg. } \\ & \text { Vol. Price }\end{aligned}$ 
mporte

Seller

Purchaser / End User

TRANSCANADA GAS SERVICES INC.

TransCanada Gas Services Limited

Kaztex Energy Management

TRANSCANADA GAS SERVICES INC.

TransCanada Gas Services Limited

KeySpan Gas East Corporation

TRANSCANADA GAS SERVICES INC.

TransCanada Gas Services Limited

Keyspan Ravenswood Inc.

TRANSCANADA GAS SERVICES INC.

TransCanada Gas Services Limited

Michigan Consolidated Gas Company

TRANSCANADA GAS SERVICES INC.

TransCanada Gas Services Limited

Michigan Energy Exchange

TRANSCANADA GAS SERVICES INC.

TransCanada Gas Services Limited

Milford Power, L.P.

Waddington, NY

$0 \quad$ N/A

$92 \quad 4.64$

o N/A

$92 \quad 4.64$

TRANSCANADA GAS SERVICES INC.

TransCanada Gas Services Limited

National Fuel Gas Distribution

Niagara Falls, NY

$14 \quad 4.12$

$14 \quad 4.51$

$10 \quad 5.30$

$38 \quad 4.56$

TRANSCANADA GAS SERVICES INC TransCanada Gas Services Limited National Fuel Gas Distribution

Noyes, Minnesota

$29 \quad 3.81$

$32 \quad 3.65$

$0 \quad$ N/A

$61 \quad 3.73$

TRANSCANADA GAS SERVICES INC. TransCanada Gas Services Limited National Fuel Resources

Niagara Falls, NY

$10 \quad 4.12$

$7 \quad 4.51$

46

5.30

$62 \quad 5.03$

TRANSCANADA GAS SERVICES INC. TransCanada Gas Services Limited National Fuel Resources

Noyes, Minnesota

$2 \quad 3.81$

$3 \quad 3.65$

$5 \quad 4.83$

$10 \quad 4.25$

TRANSCANADA GAS SERVICES INC. TransCanada Gas Services Limited

Nevada Power Company

Eastport, Idaho

$46 \quad 3.77$

$32 \quad 3.27$

$33 \quad 4.96$

111

3.98

TRANSCANADA GAS SERVICES INC. TransCanada Gas Services Limited

New Jersey Natural Gas
Waddington, NY
$11 \quad 3.88$
$0 \quad$ N/A

11 


\section{SHORT-TERM SALES \\ YEAR: 2000 Quarter: Three \\ Estimated Volumes (MMCF) \& Prices (\$ / MMBTU)}

Importer

Seller

Purchaser/End User

TRANSCANADA GAS SERVICES INC.

TransCanada Gas Services Limited

New York State Electric \& Gas Corporation

TRANSCANADA GAS SERVICES INC.

TransCanada Gas Services Limited

Northeast Gas Markets

TRANSCANADA GAS SERVICES INC.

TransCanada Gas Services Limited

PG\&E Energy Trading

TRANSCANADA GAS SERVICES INC.

TransCanada Gas Services Limited

PG\&E Energy Trading

TRANSCANADA GAS SERVICES INC.

TransCanada Gas Services Limited

PG\&E Energy Trading

TRANSCANADA GAS SERVICES INC.

TransCanada Gas Services Limited

PG\&E Energy Trading

TRANSCANADA GAS SERVICES INC.

TransCanada Gas Services Limited

Pancanadian Energy Services

TRANSCANADA GAS SERVICES INC.

TransCanada Gas Services Limited

Pancanadian Energy Services

TRANSCANADA GAS SERVICES INC.

TransCanada Gas Services Limited

Pawtucket Power Associates

TRANSCANADA GAS SERVICES INC.

TransCanada Gas Services Limited

Petrocom Energy Group Ltd.

TRANSCANADA GAS SERVICES INC.

TransCanada Gas Services Limited

Portland General Electric Company

TRANSCANADA GAS SERVICES INC

TransCanada Gas Services Limited

Proliance Energy, LLC
Waddington, NY

- N/A

$1 \quad 4.64$

o $\quad 5.44$

$1 \quad 4.71$

Waddington, NY

$7 \quad 3.88$

$7 \quad 4.64$

o N/A

$13 \quad 4.26$

Point
of
Entry

Noyes, Minnesota

Waddington, NY

$855 \quad 3.88$

$0 \quad$ N/A

$0 \quad$ N/A

$855 \quad 3.88$

Eastport, Idaho

$229 \quad 3.77$

$177 \quad 3.27$

$194 \quad 4.96$

$600 \quad 4.01$

Sumas, Washington

- N/A

o N/A

$7 \quad 4.96$

$7 \quad 4.96$

Noyes, Minnesota

$151 \quad 3.81$

$0 \quad$ N/A

153

3.81

Sumas, Washington

$21 \quad 3.49$

$34 \quad 3.34$

o N/A

$55 \quad 3.40$
Noyes, Minnesota
$0 \quad$ N/A

$18 \quad 4.83$

$18 \quad 4.83$ 


\section{SHORT-TERM SALES \\ YEAR: 2000 Quarter: Three \\ Estimated Volumes (MMCF) \& Prices (\$ / MMBTU)}

Importer

Seller

Purchaser / End User

TRANSCANADA GAS SERVICES INC.

TransCanada Gas Services Limited

Questar Pipeline Company

TRANSCANADA GAS SERVICES INC.

TransCanada Gas Services Limited

Reliant Energy Services, Inc.

TRANSCANADA GAS SERVICES INC.

TransCanada Gas Services Limited

Reliant Energy Services, Inc.

TRANSCANADA GAS SERVICES INC

TransCanada Gas Services Limited

Renaissance Energy (U.S.) inc.

TRANSCANADA GAS SERVICES INC.

TransCanada Gas Services Limited

SCANA Energy Marketing

TRANSCANADA GAS SERVICES INC.

TransCanada Gas Services Limited

Sempra Energy Trading Corp.

TRANSCANADA GAS SERVICES INC. TransCanada Gas Services Limited

Sempra Energy Trading Corp.

TRANSCANADA GAS SERVICES INC.

TransCanada Gas Services Limited

Sempra Energy Trading Corp.

TRANSCANADA GAS SERVICES INC.

TransCanada Gas Services Limited

Sempra Energy Trading Corp.

TRANSCANADA GAS SERVICES INC.

TransCanada Gas Services Limited

Sierra Pacíic Power Company

Sumas, Washington

TRANSCANADA GAS SERVICES INC. TransCanada Gas Services Limited Sithe Energy

Waddington, NY

.

TRANSCANADA GAS SERVICES INC. TransCanada Gas Services Limited

South Jersey Resources Inc.
$6 \quad 3.49$

$0 \quad$ N/A

$0 \quad N / A$

$6 \quad 3.49$

$147 \quad 4.64$

- N/A

152

4.61

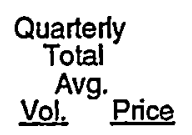

$2 \quad 3.49$

5024.00

$2739 \quad 4.15$

14.83

$\begin{array}{llllllll}0 & \text { N/A } & 142 & 3.65 & 0 & \text { N/A } & 142 & 3.65\end{array}$

$21 \quad 3.77$

$31 \quad 4.27$

$\begin{array}{llllllll}0 & \text { N/A } & 142 & 3.65 & 0 & \text { N/A } & 142 & 3.65\end{array}$

$\begin{array}{llllll}3.49 & 0 & N / A & 0 & N / A & 6\end{array}$

$\begin{array}{llllllll}33 & 3.88 & 129 & 4.64 & 35 & 5.44 & 198 & 4.65\end{array}$

$\begin{array}{llllllll}0 & \text { N/A } & 1 & 4.51 & 20 & 5.30 & 20 & 5.27\end{array}$
Niagara Falls, NY 


\section{SHORT-TERM SALES \\ YEAR: 2000 Quarter: Three \\ Estimated Volumes (MMCF) \& Prices (\$ / MMBTU)}

Importer

Seller

Purchaser / End User

TRANSCANADA GAS SERVICES INC.

TransCanada Gas Services Limited

Southem Califomia Gas Company

TRANSCANADA GAS SERVICES INC.

TransCanada Gas Services Limited

Southem Company Energy

TRANSCANADA GAS SERVICES INC.

TransCanada Gas Services Limited

Sprague Energy

TRANSCANADA GAS SERVICES INC.

TransCanada Gas Services Limited

TXU Energy Trading Company

TRANSCANADA GAS SERVICES INC. TransCanada Gas Services Limited

Texaco Natural Gas

TRANSCANADA GAS SERVICES INC. TransCanada Gas Services Limited

Texaco Natural Gas

TRANSCANADA GAS SERVICES INC TransCanada Gas Services Limited

Texaco Natural Gas

TRANSCANADA GAS SERVICES INC. TransCanada Gas Services Limited

Texaco Natural Gas

TRANSCANADA GAS SERVICES INC. TransCanada Gas Services Limited

Texex Energy Partners

TRANSCANADA GAS SERVICES INC. TransCanada Gas Services Limited TransCanada Energy Marketing USA

TRANSCANADA GAS SERVICES INC. TransCanada Gas Services Limited TransCanada Energy Marketing USA

TRANSCANADA GAS SERVICES INC. TransCanada Gas Services Limited TransCanada Gas Services
Point

Ef Entry

Vol. July Price

September

Quarterly

Total

Vol. Price

Eastport, Idaho

$\begin{array}{ll}16 & 3.77\end{array}$

$0 \quad$ N/A

$11 \quad 4.96$

$27 \quad 4.25$

Waddington, NY

o N/A

$0 \quad$ N/A

$15 \quad 5.44$

15

5.44

Waddington, NY

$\begin{array}{ll}9 & 3.88\end{array}$

o N/A

$0 \quad$ N/A

$\begin{array}{ll}9 & 3.88\end{array}$

Waddington, NY

- N/A

$72 \quad 4.64$

$0 \quad$ N/A

$72 \quad 4.64$

Eastport, Idaho

$0 \quad$ N/A

$5 \quad 3.27$

$22 \quad 4.96$

27 . 4.66

Niagara Falls, NY

- N/A

$5 \quad 4.51$

$45 \quad 5.30$

$50 \quad 5.22$

Noyes, Minnesota

- N/A

$71 \quad 3.65$

$110 \quad 4.83$

181

4.37

Sumas, Washington

$30 \quad 3.49$

o N/A

$0 \quad$ N/A

30

3.49

Waddington, NY

$23 \quad 3.88$

0 N/A

o N/A

23

3.88

Noyes, Minnesota

28

3.81

$204 \quad 3.75$

128

4.83

360

4.14

Port of Morgan, MT

- N/A

$108 \quad 3.40$

158

4.14

266

3.84

Eastport, Idaho $\begin{array}{lllll}73 & 3.44 & 108 & 3.28\end{array}$ 


\section{SHORT-TERM SALES \\ YEAR: 2000 Quarter: Three \\ Estimated Volumes (MMCF) \& Prices (\$ / MMBTU)}

Importer

Seller

Purchaser / End User

TRANSCANADA GAS SERVICES INC.

TransCanada Gas Services Limited

United States Gypsum

TRANSCANADA GAS SERVICES INC.

TransCanada Gas Services Limited

Utilicorp United

TRANSCANADA GAS SERVICES INC.

TransCanada Gas Services Limited

WiCOR Energy Service Company

TRANSCANADA GAS SERVICES INC.

TransCanada Gas Services Limited

WPS Energy Services, Inc.

TRANSCANADA GAS SERVICES INC

TransCanada Gas Services Limited

Western Gas Resources

Point

Entry

Vol. July Price

August

Vol. Price

September

Vol. Price

Quarterly

Total

Vol. ${ }^{\text {Avg. }}$ Price

Waddington, NY

$0 \quad$ N/A

$44 \quad 4.64$

- N/A

$44 \quad 4.64$

Noyes, Minnesota

$\begin{array}{llll}0 & \text { N/A } & 71 & 3.65\end{array}$

$0 \quad$ N/A

71

3.65

Noyes, Minnesota

$118 \quad 3.81$

$91 \quad 3.65$

$9 \quad 4.83$

218

3.78

Noyes, Minnesota

$29 \quad 3.81$

$33 \quad 3.65$

56

4.83

118

4.25

Eastport, Idaho

$1 \quad 3.77$

$0 \quad$ N/A

$0 \quad$ N/A

$1 \quad 3.77$

TRANSCANADA GAS SERVICES INC.

TransCanada Gas Services Limited

Western Gas Resources

Waddington, NY

$11 \quad 3.88$

$0 \quad$ N/A

$0 \quad$ N/A

$11 \quad 3.88$

TRANSCANADA GAS SERVICES INC.

TransCanada Gas Services Limited

Wisconsin Electric

Noyes, Minnesota

O N/A

$5 \quad 3.65$

$0 \quad$ N/A

$5 \quad 3.65$

TRANSCANADA GAS SERVICES INC.

TransCanada Gas Services Limited

Wisconsin Fuel \& Light

Noyes, Minnesota

$18 \quad 3.81$

$0 \quad$ N/A

- N/A

18

3.81

TRANSCANADA GAS SERVICES INC.

TransCanada Gas Services Limited

Wisconsin Gas

Noyes, Minnesota

$339 \quad 3.81$

$32 \quad 3.65$

314.83

401

3.88

TRANSCANADA GAS SERVICES INC.

TransCanada Gas Services Limited

Wisconsin Power \& Light

Noyes, Minnesota

$43 \quad 3.81$

$37 \quad 3.65$

$3 \quad 4.83$

$83 \quad 3.77$

TRANSCO ENERGY MARKETING COMPANY

Altrade

Various NY State Markets

Niagara Falls, NY

- N/A

$80 \quad 4.56$

$5 \quad 5.41$

85

4.61

TRANSCO ENERGY MARKETING COMPANY CXY Marketing

Various NY State Markets
Niagara Falls, NY

$308 \quad 4.48$

$7 \quad 4.35$

$0 \quad N / A$

314

4.48 


\section{SHORT-TERM SALES}

YEAR: 2000 Quarter: Three

Estimated Volumes (MMCF) \& Prices (\$/MMBTU)

Importer

Seller

Purchaser / End User

TRANSCO ENERGY MARKETING COMPANY

Coral Energy Canada Inc.

Vanous NY State Markets

Niagara Falls, NY

TRANSCO ENERGY MARKETING COMPANY

Duke Energy Marketing Canada

Various NY State Markets

Niagara Falls, NY

TRANSCO ENERGY MARKETING COMPANY El Paso Energy Markeing Canada

Various NY State Markets

Niagara Falls, NY

TRANSCO ENERGY MARKETING COMPANY Enron North America Corp.

Various NY State Markets

Niagara Falls, NY

TRANSCO ENERGY MARKETING COMPANY Indeck-Oswego, L.P.

Various NY State Markets

Niagara Falls, NY

TRANSCO ENERGY MARKETING COMPANY Indeck-Yerkes Energy Services

Various NY State Markets

Niagara Falls, NY

TRANSCO ENERGY MARKETING COMPANY PG\&E Energy Trading

Various NY State Markets

Niagara Falls, NY

$0 \quad$ N/A

$5 \quad 4.33$

O N/A

5

4.33

TRANSCO ENERGY MARKETING COMPANY

ProGas Limited

Various NY State Markets

Niagara Falls, NY

$165 \quad 4.12$

$165 \quad 4.51$

o N/A

330

4.32

TRANSCO ENERGY MARKETING COMPANY

Renaissance Energy Ltd.

Various NY State Markets

Niagara Falls, NY

$112 \quad 4.10$

$75 \quad 4.55$

$85 \quad 4.96$

$272 \quad 4.49$

TRANSCO ENERGY MARKETING COMPANY

Sempra Energy Trading

Various NY State Markets

Niagara Falls, NY

$154 \quad 4.52$

$5 \quad 4.53$

$149 \quad 4.77$

$308 \quad 4.64$

TRANSCO ENERGY MARKETING COMPANY

TXU

Various NY State Markets

Niagara Falls, NY

TRANSCO ENERGY MARKETING COMPANY

Texex

Various NY State Markets

Niagara Falls, NY
$461 \quad 4.50$

$0 \quad$ N/A

$0 \quad$ N/A

$461 \quad 4.50$

$\begin{array}{llll}0 & \text { N/A } & 70 & 4.48\end{array}$

O N/A

$70 \quad 4.48$ 


\section{SHORT-TERM SALES \\ YEAR: 2000 Quarter: Three \\ Estimated Volumes (MMCF) \& Prices (\$ / MMBTU)}

Importer

Seller

Purchaser/End User

TRANSCO ENERGY MARKETING COMPANY

TransCanada Gas Services Limited

Various NY State Markets

Niagara Falls, NY

TXU ENERGY TRADING COMPANY

Duke Energy Marketing Canada

Various CA State Markets

TXU ENERGY TRADING COMPANY

TXU

Various CA State Markets

TXU ENERGY TRADING COMPANY

TXU

Various NY State Markets

UNITED STATES GYPSUM COMPANY

Coral Energy Canada Inc.

United States Gypsum

UNITED STATES GYPSUM COMPANY

Pan-Alberta Gas Ltd.

United States Gypsum

USGEN NEW ENGLAND, INC.

PG\&E Energy Trading

PG\&E Energy Trading

UTILICORP UNITED INC.

Aquila Canada Corporation

Utlicorp United

UTILICORP UNITED INC.

CXY Marketing

Utilicorp United

UTILICORP UNITED INC.

CXY Marketing

Utilicorp United

Wartoad, Minnesota

O N/A

$0 \quad$ N/A

$5 \quad 4.96$

$5 \quad 4.96$

UTILICORP UNITED INC.

Coral Energy Canada Inc.

Utilicon United

Warroad, Minnesota

$356 \quad 4.01$

$\begin{array}{ll}7 & 3.31\end{array}$

$8 \quad 4.49$

371

4.01

UTILICORP UNITED INC.

Tenaska Marketing Canada Utilicorp United

July August
Vol. September
Pol. Price

$\begin{array}{llllllll}292 & 3.88 & 0 & \text { N/A } & 0 & \text { N/A } & 292 & 3.88\end{array}$

$\begin{array}{llllllll}33 & 4.16 & 12 & 4.76 & 832 & 4.89 & 877 & 4.86\end{array}$

$\begin{array}{llllllll}77 & 3.90 & 77 & 3.46 & 76 & 3.96 & 230 & 3.77\end{array}$

$\begin{array}{llllllll}233 & 3.90 & 237 & 3.46 & 229 & 3.96 & 699 & 3.77\end{array}$

$\begin{array}{llllllll}1913 & 4.33 & 2994 & 4.06 & 1519 & 4.80 & 6426 & 4.32\end{array}$

$\begin{array}{llllllll}356 & 3.80 & 356 & 3.30 & 345 & 3.70 & 1058 & 3.60\end{array}$

$\begin{array}{llllllll}583 & 4.24 & 583 & 3.65 & 564 & 4.46 & 1730 & 4.11\end{array}$

$\begin{array}{lllllllll}\text { - Warroad, Minnesota } & 11 & 3.38 & 5 & 3.37 & 5 & 4.85 & 21 & 3.74\end{array}$ 
SHORT-TERM SALES

YEAR: 2000 Quarter: Three

Estimated Volumes (MMCF) \& Prices (\$ / MMBTU)
Importer

Seller

Purchaser/End User

UTILICORP UNITED INC.

TransCanada Gas Services Limited Utilicom United

UTILICORP UNITED INC.

TransCanada Gas Services Limited Utilicorp United

UTILICORP UNITED INC.

TransCanada Pipelines Limited

Utilicorp United

UTILICORP UNITED INC.

TransCanada Pipelines Limited Utilicorp United

VERMONT GAS SYSTEMS, INC.

Coral Energy Canada Inc.

Vermont Gas Systems, Inc.

VERMONT GAS SYSTEMS, INC.

TransCanada Gas Services Limited

Vermont Gas Systems, Inc.

WISCONSIN GAS COMPANY

CXY Marketing

Wisconsin Gas

WISCONSIN GAS COMPANY

Coral Energy Canada Inc.

Wisconsin Gas

WISCONSIN GAS COMPANY

Duke Energy Marketing Canada

Wisconsin Gas

WISCONSIN GAS COMPANY

ProGas Limited

Wisconsin Gas

WISCONSIN GAS COMPANY

Renaissance Energy Ltd.

Wisconsin Gas

WISCONSIN GAS COMPANY

Tenaska Marketing Canada

Wisconsin Gas
Point
of
Entry

Noyes, Minnesota

Warroad, Minnesota

Noyes, Minnesota

o N/A

$\begin{array}{ll}15 & 3.57\end{array}$

O N/A

$2 \quad 3.56$

- N/A

$2 \quad 3.56$

Highgate Spr., VT

$77 \quad 4.04$

$72 \quad 4.42$

$64 \quad 5.19$

$213 \quad 4.52$

Highgate Spr., VT

$62 \quad 4.25$

$47 \quad 4.60$

$88 \quad 5.45$

196

4.87

Noyes, Minnesota

$241 \quad 3.28$

- N/A

$0 \quad$ N/A

24

3.28

Noyes, Minnesota

28

3.35

- N/A

$0 \quad$ N/A

281

3.35

Noyes, Minnesota

$16 \quad 3.55$

- N/A

$0 \quad$ N/A

$16 \quad 3.55$

Noyes, Minnesota

$\begin{array}{ll}733 & 3.43\end{array}$

- N/A

o N/A

$\begin{array}{ll}733 & 3.43\end{array}$

Noyes, Minnesota

$192 \quad 3.36$

- N/A

- N/A

192

3.36

Noyes, Minnesota

$55 \quad 3.35$

o N/A

$0 \quad$ N/A

$55 \quad 3.35$

$55 \quad 3.35$


SHORT-TERM SALES

YEAR: 2000 Quarter: Three

Estimated Volumes (MMCF) \& Prices (\$ / MMBTU)
Importer

Seller

Purchaser / End User

WISCONSIN GAS COMPANY

TransCanada Gas Services Limited

Wisconsin Gas

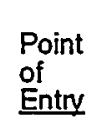

Noyes, Minnesota

\author{
Vol. July Price
}

August
Vol. Price

$307 \quad 3.38$

o N/A

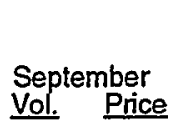
Quarterly
Total
Vol. ${ }^{\text {Avg. }}$ Price

\author{
Vol \\ Price
}

o N/A

307

3.38 


\section{SHORT-TERM EXPORTERS}

Estimated Volumes (MMCF)

\begin{tabular}{|c|c|c|c|c|c|}
\hline & $\begin{array}{l}1999 \\
\text { Jul.-Sep. }\end{array}$ & $\begin{array}{ll}1999 \\
\text { Oct.Dec. }\end{array}$ & $\begin{array}{l}2000 \\
\text { Jan.-Mar. }\end{array}$ & $\begin{array}{l}2000 \\
\text { Apr.-Jun. }\end{array}$ & $\begin{array}{l}2000 \\
\text { Jul.-Sep. }\end{array}$ \\
\hline AGAVE ENERGY COMPANY & 0 & 0 & 0 & 0 & 58 \\
\hline APPLIED LNG TECHNOLOGIES USA, L.L.C. & 7 & 69 & 118 & 91 & 86 \\
\hline BC GAS UTILITY LTD. & 0 & 0 & 456 & 0 & 0 \\
\hline BIG SKY GAS MARKETING & 598 & 260 & 48 & 64 & 312 \\
\hline BURLINGTON RESOURCES TRADING INC. & 184 & 0 & 0 & 0 & 0 \\
\hline COENERGY TRADING COMPANY & 0 & 4388 & 5329 & 0 & 30 \\
\hline CONOCOINC. & 0 & 0 & 0 & 0 & 676 \\
\hline CONSUMERS' GAS COMPANY LIMITED & 0 & 0 & 2976 & 1447 & 0 \\
\hline COOK INLET ENERGY SUPPLY, L.P. & 0 & 0 & 0 & 0 & 3340 \\
\hline CORAL ENERGY RESOURCES, L.P. & 365 & 0 & 0 & 0 & 0 \\
\hline DIST. DE GAS NATURAL DE MEXICALI & 1017 & 1004 & 1218 & 1074 & 910 \\
\hline DUKE ENERGY TRADING AND MKTG SERVICES L.L.C. & 1353 & 303 & 0 & 0 & 0 \\
\hline DYNEGY MARKETING \& TRADE & 213 & 916 & 714 & 74 & 286 \\
\hline EL PASO ENERGY MARKETING COMPANY & 1184 & 621 & 27 & 0 & 759 \\
\hline ENER-SON OF U.S.A. & 54 & 11 & 0 & 0 & 0 \\
\hline ENRON NORTH AMERICA CORPORATION & 0 & 0 & 0 & 0 & 85 \\
\hline GASODUCTO DE ROSARITO, S. DE R.L. DE C.V. & 0 & 0 & 0 & 71 & 5418 \\
\hline MARATHON OIL COMPANY & 0 & 0 & 0 & 0 & 203 \\
\hline MEXICANA DE COBRE, S.A. DE C.V. & 1038 & 570 & 706 & 716 & 841 \\
\hline PEMEX GAS & 11575 & 10641 & 17466 & 24334 & 18590 \\
\hline PG\&E TEXAS INDUSTRIAL ENERGY, L.P. & 0 & 0 & 0 & 573 & 0 \\
\hline PREMSTAR ENERGY CANADA, LTD. & 0 & 450 & 650 & 1374 & 2662 \\
\hline STAR NATURAL GAS COMPANY & 0 & 22 & 1355 & 0 & 0 \\
\hline TENASKA MARKETING VENTURES & 0 & 3416 & 4330 & 1600 & 571 \\
\hline THE MONTANA POWER TRADING \& MARKETING COM & APANYO & 14 & 679 & 0 & 0 \\
\hline TRISTAR GAS MARKETING COMPANY & 0 & 0 & 0 & 0 & 348 \\
\hline UNION GAS LIMITED & 5367 & 7537 & 8576 & 6648 & 8432 \\
\hline WEST TEXAS GAS, INC. & 454 & 541 & 581 & 506 & 445 \\
\hline WGR CANADA, INC. & 0 & 0 & 0 & 9 & 0 \\
\hline XENO, INC. & 0 & 0 & .0 & 0 & 264 \\
\hline IOTALS & $\underline{23408}$ & $\underline{30761}$ & 45229 & 38580 & 44316 \\
\hline
\end{tabular}




\section{PURCHASERS OF SHORT-TERM EXPORTS Estimated Volumes Exported (MMCF)}

ACCO

Altrade

Aquila Canada Corporation

Baja Oriente

BC Gas Inc.

C\&D Die Casting

Cibola Canada

Clayton Energy Corporation

CoEnergy Trading Company

\section{Conagas}

Cris-P

Distribuidora de Gas Natural de Mexicali

Duke Energy Marketing Canada

Dynegy Canada Inc.

El Paso Energy Marketing Canada

Engage Energy Canada L.P.

Enogex Services

Enron Capital \& Trade Resources Canada

KN Marketing L.P.

Koch Energy Trading

Mexcobre

MidAmerican Energy Company

Natural Gas Clearinghouse Canada, Inc.

Northem Natural Gas

OGE Energy Resources

Pancanadian Energy Services

PEMEX

1999
Jul.-Sep.

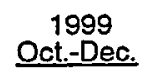

2000
Jan.-Mar.

2000

Apr.-Jun. Jul-Sep

PFALTZGRAFF

Premstar Energy Canada Ltd.

Reliant Energy Services, Inc.

Renaissance Energy Ltd.

San Francisco Tile

Sempra Energy Trading Corp.

Tenaska Marketing Ventures

TransCanada Gas Services Limited

TransCanada Pipelines Limited

TXU Energy Trading Company

Various Alberta Customers

Various Ontario Customers

Various Saskatchewan Customers

Weiseriock

WGR Canada, Inc.

\begin{tabular}{|c|c|c|c|c|}
\hline 21 & 13 & 13 & 8 & 8 \\
\hline 0 & 0 & 155 & 0 & 0 \\
\hline 0 & 0 & 6 & 0 & 0 \\
\hline 3 & 24 & 44 & 39 & 38 \\
\hline 0 & 0 & 456 & 0 & 0 \\
\hline 0 & 0 & 2 & 1 & 3 \\
\hline 0 & 41 & 79 & 0 & 0 \\
\hline 0 & 0 & 4 & 0 & 0 \\
\hline 0 & 4388 & 5329 & 0 & 30 \\
\hline 454 & 541 & 581 & 506 & 445 \\
\hline 4 & 19 & 20 & 9 & 2 \\
\hline 1017 & 1004 & 1218 & 1074 & 910 \\
\hline 0 & 0 & 5 & 0. & 0 \\
\hline 0 & 759 & 348 & .0 & 0 \\
\hline 1104 & 621 & 27 & 0 & 3 \\
\hline 0 & 0 & 0 & 0 & 30 \\
\hline 0 & 10 & 0 & 0 & 0 \\
\hline 0 & 10 & 1045 & 0 & 0 \\
\hline 0 & 0 & 169 & 0 & 0 \\
\hline 0 & 2 & 70 & 0 & 0 \\
\hline 1038 & 570 & 706 & 716 & 841 \\
\hline 0 & 20 & 5 & 0 & 0 \\
\hline 0 & 0 & 2 & 0 & 0 \\
\hline 0 & 0 & 0 & 74 & 130 \\
\hline 0 & 0 & 0 & 0 & 120 \\
\hline 0 & 0 & 4 & 0 & 0 \\
\hline 3770 & 10944 & 17466 & 24978 & 29475 \\
\hline 28 & 22 & 31 & 32 & 36 \\
\hline 0 & 450 & 650 & 1374 & 2662 \\
\hline 0 & 23 & 7 & 0 & 0 \\
\hline 0 & 0 & 0 & 0 & 264 \\
\hline 4 & 2 & 4 & 2 & 0 \\
\hline 0 & 55 & 155 & 0 & 0 \\
\hline 0 & 6 & 0 & 0 & 0 \\
\hline 0 & 12 & 0 & 0 & 0 \\
\hline 0 & 0 & 0 & 0 & 6 \\
\hline 0 & 0 & 14 & 0 & 0 \\
\hline 0 & 14 & 679 & 0 & 0 \\
\hline 5367 & 10952 & 15881 & 9694 & 9003 \\
\hline 598 & 260 & 48 & 64 & 312 \\
\hline 0 & 0 & 4 & 0 & 0 \\
\hline 0 & 0 & 0 & 9 & 0 \\
\hline 3408 & 30761 & 45229 & 38580 & 44316 \\
\hline
\end{tabular}

Totals 
SHORT-TERM SALES

YEAR: 2000 Quarter: Three

Estimated Volumes (MMCF) \& Prices (\$/MMBTU)

Exporter

Seller

Purchaser / End User

Point

Exit

El Paso, Texas

Nogales, Arizona

$3 \quad 5.82$

$3 \quad 5.82$

14. 5.82

$13 \quad 5.82$

$11 \quad 5.82$

$38 \quad 5.82$

Applied LNG Technologies USA, L.L.C.

Baja Oriente

APPLIED LNG TECHNOLOGIES USA, L.L.C.

Applied LNG Technologies USA, LL.C.

C\&D Die Casting

Nogales, Arizona

15.82

$0 \quad 5.82$

$2 \quad 5.82$

$3 \quad 5.82$

APPLIED LNG TECHNOLOGIES USA, L.L.C.

Applied LNG Technologies USA, L.L.C.
Cris-P

Nogales, Arizona

- N/A

- N/A

$2 \quad 5.82$

$2 \quad 5.82$

APPLIED LNG TECHNOLOGIES USA, L.L.C. Applied LNG Technologies USA, L.L.C.

PFALTZGRAFF

Nogales, Arizona

$11 \quad 5.82$

$13 \quad 5.82$

$12 \quad 5.82$

$36 \quad 5.82$

BIG SKY GAS MARKETING

Big Sky Gas Marketing

Various Saskatchewan Customers

Havre, Montana

$98 \quad 3.20$

$141 \quad 3.31$

$\begin{array}{ll}73 & 4.38\end{array}$

$312 \quad 3.53$

COENERGY TRADING COMPANY

CoEnergy Trading Company

CoEnergy Trading Company

Detroit, Michigan

Alamo, Texas

Douglas, Arizona

$34 \quad 3.61$

$0 \quad$ N/A

$62 \quad 4.66$

$96 \quad 4.29$

COOKINLET ENERGY SUPPLY, L.P.

Cook Inlet Energy Supply

PEMEX

Clint, Texas

$1216 \quad 4.35$

627

4.50

2280

4.28

COOK INLET ENERGY SUPPLY, L.P. Cook Inlet Energy Supply

PEMEX
Hidalgo, Texas
368

3.79

$392 \quad 4.06$

$300 \quad 4.48$

$1060 \quad 4.09$ 
Page -118

SHORT-TERM SALES

YEAR: 2000 Quarter: Three

Estimated Volumes (MMCF) \& Prices (\$ / MMBTU)
Exporter

Seller

Purchaser / End User

DIST. DE GAS NATURAL DE MEXICALI

Coral Energy Resources

Distribuidora de Gas Natural de Mexicali

DIST. DE GAS NATURAL DE MEXICALI

Engage Energy US, L.P.

Distribuidora de Gas Natural de Mexicali

DYNEGY MARKETING \& TRADE

Dynegy Marketing \& Trade

Engage Energy Canada L.P.

DYNEGY MARKETING \& TRADE

Dynegy Marketing \& Trade

Northem Natural Gas

DYNEGY MARKETING \& TRADE

Dynegy Marketing \& Trade

OGE Energy Resources

DYNEGY MARKETING \& TRADE

Dynegy Marketing \& Trade

TransCanada Pipelines Limited

EL PASO ENERGY MARKETING COMPANY

El Paso Energy Marketing Company

El Paso Energy Marketing Canada

EL PASO ENERGY MARKETING COMPANY

El Paso Energy Marketing Company

PEMEX

EL PASO ENERGY MARKETING COMPANY

El Paso Energy Marketing Company

PEMEX

El Paso, Texas

ENRON NORTH AMERICA CORPORATION

Enron North America Corp.

PEMEX

Hidalgo, Texas

GASODUCTO DE ROSARITO, S. DE R.L. DE C.V.

Sempra Energy Trading

PEMEX

Otay Mesa, CA

MARATHON OIL COMPANY

Marathon Oil Company

PEMEX

El Paso, Texas
24.05

$5 \quad 3.80$

$0 \quad$ N/A

$\begin{array}{ll}7 & 3.87\end{array}$

July
Vol. Pugust September

Quarterly

Total

Avg.

Vol. Price

$\begin{array}{llll}324 & 5.23 & 321 & 4.85\end{array}$

$0 \quad$ N/A

$645 \quad 5.04$

$130 \quad 4.26$

- N/A

o N/A

130

4.26

$120 \quad 4.26$

$0 \quad$ N/A

$0 \quad$ N/A

$120 \quad 4.26$

$6 \quad 4.23$

- $0 \quad$ N/A

$0 \quad$ N/A

$6 \quad 4.23$

$30 \quad 3.87$

$0 \quad$ N/A

720

4.48

750

4.46

$\begin{array}{llll}35 & 4.12 & 50 & 4.16\end{array}$

$0 \quad 4.20$

85

4.14

$1388 \quad 4.91$

$2142 \quad 4,49$

1888

6.31

5418

5.23

$0 \quad$ N/A $\quad 188$

$15 \quad 4.76$

203

3.75 


\section{SHORT-TERM SALES \\ YEAR: 2000 Quarter: Three \\ Estimated Volumes (MMCF) \& Prices (\$ / MMBTU)}

Exporter

Seller

Purchaser / End User

MEXICANA DE COBRE, S.A. DE C.V.

MGI Supply

Mexcobre

\section{PEMEX GAS \\ MGI Supply \\ PEMEX}

\section{PEMEX GAS}

MGI Supply

PEMEX

\section{PEMEX GAS}

MGI Supply

PEMEX

PEMEX GAS

MGI Supply

PEMEX

PREMSTAR ENERGY CANADA, LTD.

CMS Marketing

Premstar Energy Canada Ltd.

PREMSTAR ENERGY CANADA, LTD.

Premstar Energy

Premstar Energy Canada Ltd.

TENASKA MARKETING VENTURES

Tenaska Marketing Ventures

Various Ontario Customers

TRISTAR GAS MARKETING COMPANY Tristar Gas Marketing Company

PEMEX

UNION GAS LIMITED

Amoco Canada Petroleum Company Ltd.

Various Ontario Customers

UNION GAS LIMITED

Anadarko Trading Company

Various Ontario Customers

UNION GAS LIMITED

CMS Marketing

Various Ontario Customers
Detroit, Michigan

Point

Douglas, Arizona

Alamo, Texas

Clint, Texas

Douglas, Arizona

El Paso, Texas

St. Clair, Michigan

El Paso, Texas

Detroit, Michigan

Detroit, Michigan

Detroit, Michigan
- N/A

$0 \quad$ N/A

$50 \quad 5.12$

50

5.12

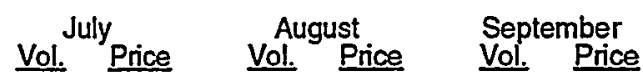

Quartenty

Total

Avg.

Vol. Price

$\begin{array}{llllllll}1865 & 3.79 & 2862 & 3.99 & 1705 & 4.49 & 6432 & 4.06\end{array}$

$\begin{array}{llllllll}3235 & 4.92 & 2833 & 4.16 & 3010 & 4.91 & 9078 & 4.68\end{array}$

$\begin{array}{llllllll}750 & 4.34 & 729 & 4.23 & 760 & 4.88 & 2239 & 4.49\end{array}$

$\begin{array}{llllllll}389 & 4.63 & 202 & 4.16 & 249 & 4.83 & 840 & 4.58\end{array}$

$\begin{array}{llllllll}473 & 4.51 & 570 & 3.96 & 1569 & 5.37 & 2612 & 4.91\end{array}$

$\begin{array}{llllllll}172 & 4.00 & 288 & 4.25 & 111 & 4.25 & 571 & 4.17\end{array}$

$\begin{array}{llllllll}43 & 4.23 & 138 & 4.33 & 167 & 4.65 & 348 & 4.47\end{array}$

$\begin{array}{llllllll}0 & \text { N/A } & 945 & 3.85 & 0 & \text { N/A } & 945 & 3.85\end{array}$

$\begin{array}{llllllll}1297 & 4.29 & 931 & 3.89 & 1500 & 4.14 & 3728 & 4.13\end{array}$

$\begin{array}{llllllll}645 & 4.48 & 0 & N / A & 0 & N / A & 645 & 4.48\end{array}$ 
SHORT-TERM SALES

YEAR: 2000 Quarter: Three

Estimated Volumes (MMCF) \& Prices (\$ / MMBTU)
Exporter

Seller

Purchaser/End User

UNION GAS LIMITED

Cargill Energy Division

Various Ontario Customers

UNION GAS LIMITED

Duke Energy Marketing Canada

Various Ontario Customers

UNION GAS LIMITED

Engage Energy Canada

Various Ontario Customers

UNION GAS LIMITED

Enron Gas Marketing Canada

Various Ontario Customers

UNION GAS LIMITED

Oneok

Various Ontario Customers

UNION GAS LIMITED

Reliant Energy Services Canada, inc.

Various Ontario Customers

WEST TEXAS GAS, INC.

West Texas Gas, Inc.

Conagas

XENO, INC.

Xeno, Inc.

Renaissance Energy Ltd.
Point

Exit

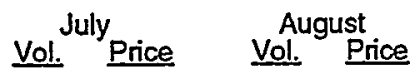

September

Vol. Price

Quarterly

Total

Vol. ${ }^{\text {Avg. }}$ Price

Detroit, Michigan

$324 \quad 4.39$

O N/A

$0 \quad$ N/A

324

4.39

Detroit, Michigan

$162 \quad 4.26$

155

3.71

150

4.51

467

4.16

Detroit, Michigan

$161 \quad 4.33$

- N/A

- N/A

161

4.33

Detroit, Michigan

- N/A

$309 \quad 3.67$

- N/A

$309 \quad 3.67$

Detroit, Michigan

$162 \quad 4.44$

155

3.65

150

4.54

$467 \quad 4.21$

Detroit, Michigan

$161 \quad 4.33$

310

3.73

915

4.55

1386

4.34

Eagle Pass, Texas

$141 \quad 4.47$

154

3.95

150

4.73

$445 \quad 4.38$

Havre, Montana
$100 \quad 3.15$

96

3.20

$\begin{array}{ll}68 & 4.45\end{array}$

$264 \quad 3.50$ 